\title{
Defect Studies in Low-Temperature-Grown GaAs
}

\author{
David Emory Bliss \\ (Ph.D. Thesis) \\ Materials Science and Mineral Engineering Department \\ University of California \\ and \\ Center for Advanced Materials \\ Materials Sciences Division \\ Lawrence Berkeley Laboratory \\ Berkeley, CA 94720
}

November 1992

This work was supported by the Director, Office of Energy Research, Office of Basic Energy Sciences, Materials Science Division of the U.S. Department of Energy under Contract No. DE-AC03-76SF00098 
Abstract
Defect Studies in Low-Temperature-Grown GaAs

by

David Emory Bliss

Doctor of Philosophy in Materials Science and Mineral Engineering

University of California at Berkeley

Professor Eugene E. Haller, Chair

An extremely high concentration of excess $A s$ is found to be incorporated in GaAs grown by molecular-beam-epitaxy at low substrate temperatures (LTMBE GaAs). The excess As exists primarily as As antisite defects, AsGa, and leads to a lesser extent of gallium vacancies, $\mathrm{V}_{\mathrm{Ga}}$. Neutral AsGa-related defects have been measured at concentrations as high as $1020 \mathrm{~cm}^{-3}$, while the $V_{G}$ a concentration is estimated to be on the order of $1019 \mathrm{~cm}^{-3}$. The neutral AsGa-related defects were measured by the characteristic infrared absorption at a wavelength of $1 \mu \mathrm{m}$. The presence of gallium vacancies, $V_{G a}$, was investigated by slow positron annihilation. Results indicate an excess concentration of $V_{G a}$ in LTMBE layers over bulk grown crystals.

The dependence of the defect concentrations on doping has been studied by the intentional introduction of the shallow-level impurities silicon and beryllium. Electrical measurements show no free carriers are generated by $n$-type or p-type doping up to concentrations of $10^{19} \mathrm{~cm}^{-3}$ with $\mathrm{Si}$ or $\mathrm{Be}$ respectively. The compensation mechanism is different for the two types of dopants. Raman measurements of the local vibrational modes of the dopant 
atoms indicate the $\mathrm{Be}$ occupies the $\mathrm{Ga}$ substitutional sites but the $\mathrm{Si}$ atom is not substitutional. Addition of $\mathrm{Si}$ induces the incorporation of more $A s G_{a}$ in the layer. As the AsGa concentration increases, the fraction the ASGa which can be photoquenched decreases. It is suggested that the metastable properties of the AsGa-related defect are influenced by neighboring defects. The fraction of photoquenchable defects correlates to defects within 3 nearest neighbor separations disrupting the metastability.

Annealing studies show that a rapid decrease in the neutral AsGa concentration occurs around $500^{\circ} \mathrm{C}$. This distinct annealing stage is similar to that found in irradiation damaged and plastically deformed GaAs, as opposed to bulk grown GaAs in which AsGa-related defects are stable up to $1100^{\circ} \mathrm{C}$. It is proposed that the lower temperature defect removal mechanism is due to the $V_{G a}$ enhanced diffusion of AsGa to arsenic precipitates. The concentration of $\mathrm{V}_{\mathrm{Ga}}$ is supersaturated and also decreases during annealing. Hence, the diffusion is modelled with a time dependent diffusivity. Analysis of isothermal annealing kinetiss for the removal of AsGa-related defects gives an enthalpy of migration of $2.0 \pm 0.3 \mathrm{eV}$ and $1.5 \pm 0.3 \mathrm{eV}$ for the $A S_{\mathrm{Ga}}$ and $V_{G a}$ respectively. The difference in enthalpy represents the difference between $\mathrm{a} G \mathrm{Ga}$ atom and an $\mathrm{As}$ atom hopping into the vacancy. The non-photoquenchable AsGarelated defects anneal away with an activation energy of $1.1 \pm 0.3 \mathrm{eV}$.

Beryllium acceptors can be activated by annealing at $800{ }^{\circ} \mathrm{C}$. The temperature difference between the annealing of defects and the activation of $\mathrm{Be}$ suggests the formation of $\mathrm{AsGa}-\mathrm{BeGa}_{\mathrm{G}}$ pairs. The $\mathrm{Si}$ donors can only be partially activated by annealing at $800^{\circ} \mathrm{C}$. 


\section{Table of Contents}

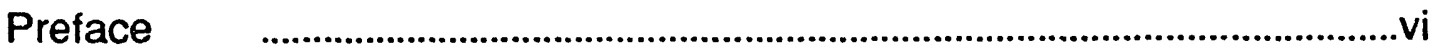

Acknowledgements...............................................................................................viii

Chapter 1. Overview of Semiconductor Technology and Review of LTMBE GaAs

1.1. Semiconductor Technology.........................................................1

1.1.1. An historical perspective ..................................................1

1.1.2. Devices, a materials perspective......................................4

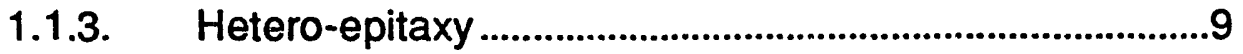

1.1.4. Defects and Devices ...................................................12

1.1.5. LTMBE Buffer Layers ......................................................14

1.2. The MBE Technique..................................................................18

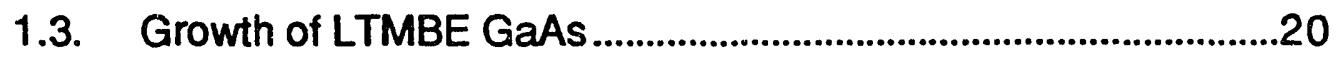

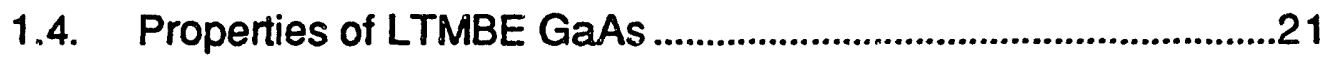

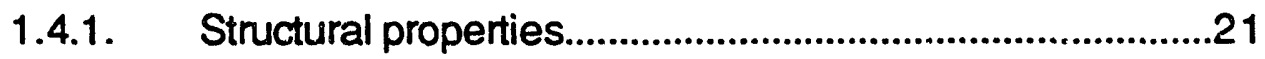

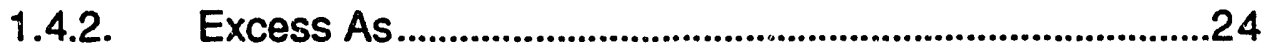

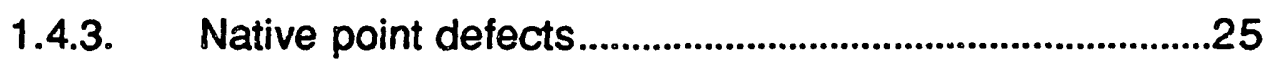

1.4.4. Metastability of AsGa-related defects............................26

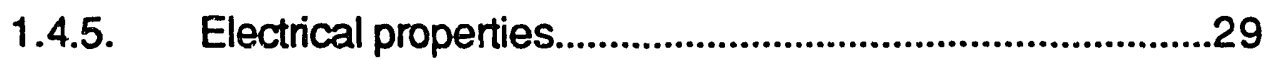

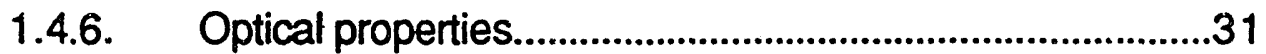

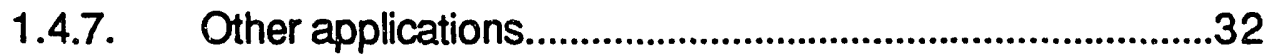

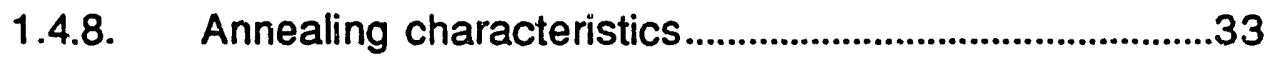

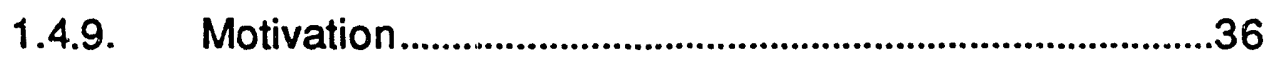

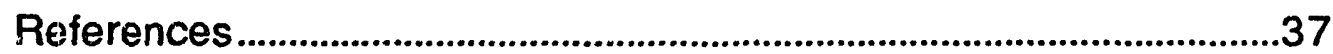

Chapter 2. Experimental Results:

As-grown LTMBE GaAs

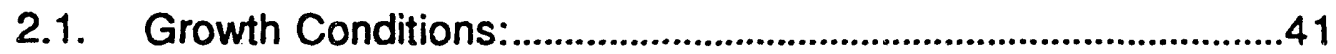

2.2. Characterization of undoped LTMBE GaAs..............................42

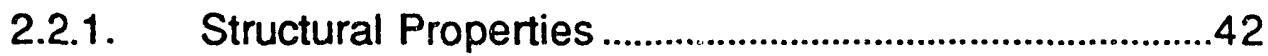

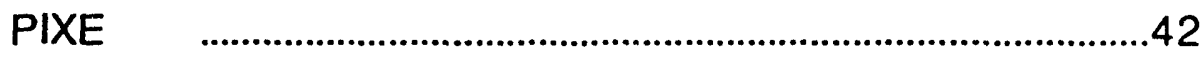

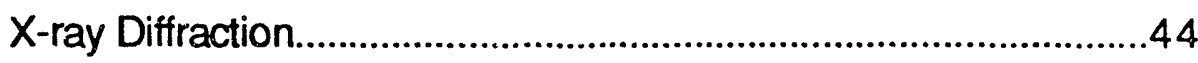


TEM

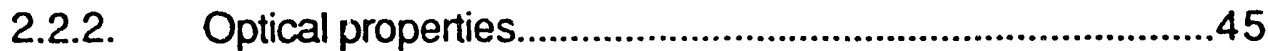

IR absorption of EL2o-like defects..............................................45

Photoquenching and thermal recovery ......................................49

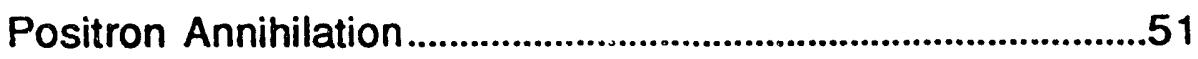

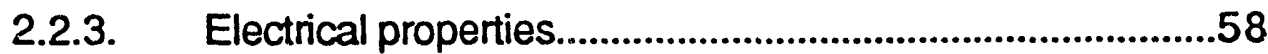

2.2.4. Hydrogen plasma passivation......................................61

2.3. Characterization of doped layers..............................................63

2.3.1. Growth conditions..................................................63

2.3.2. Secondary Ion Mass Spectrometry (SIMS).............64

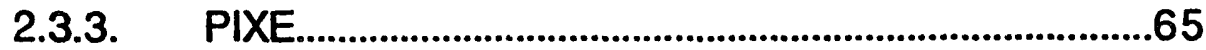

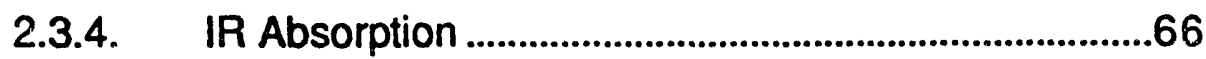

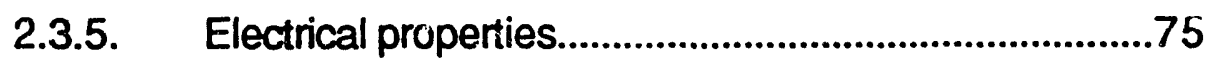

2.3.6. Local Vibrational Modes...........................................82

2.3.7. Electron paramagnetic resonance .............................86

2.3.8. Positron annihilation ....................................................89

2.3.9. Constant photocurrent characterization ....................93

2.3.10. Photoluminescence...................................................98

2.4. Summary and Concluding Comments .....................................100

References................................................................................................104

\section{Chapter 3. Annealing Characteristics of LTMBE GaAs}

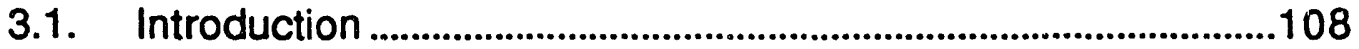

3.2. Isochronal annealing ................................................................109

3.2.1. Annealing conditions ...................................................109

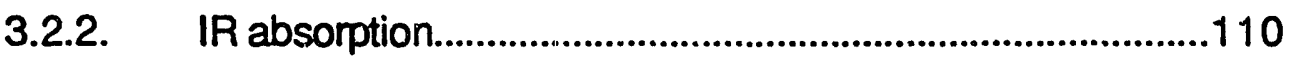

3.2.3. Electrical characterization .............................................114

Dependence of hopping conductivity on annealing temperature.............................................................114

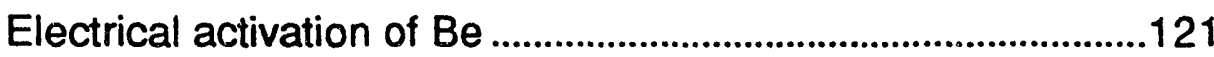

Electrical activation of $\mathrm{Si}$..........................................................123

3.3. Isothermal annealing experiments ...............................................128

3.3.1. Isothermal annealing conditions....................................129

3.3.2. Isothermal Annealing Results......................................129

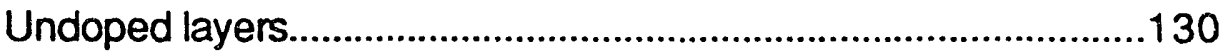


Sources of error in the recovery transient................................132

Doped layers ...........................................................................132

3.3.3. Model of VGa enhanced diffusion of

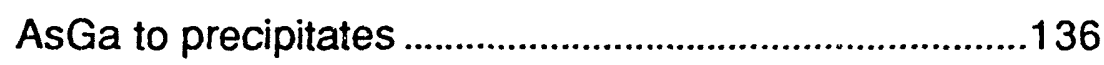

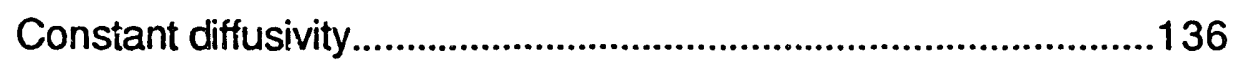

Time dependent diffusivity .......................................................141

Fitting the diffusion model to the data ......................................144

Electronic energy level of NPQ AsGa-related defect ................146

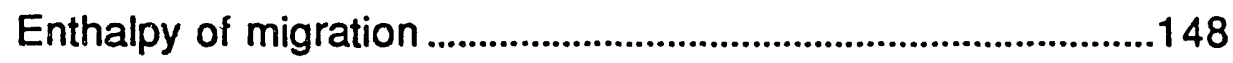

Concentration dependent diffusion ...........................................154

Concetration of VGa and the entropy of migration ...................158

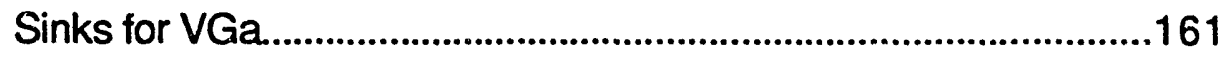

3.4. Summary and Conclusions ...................................................164

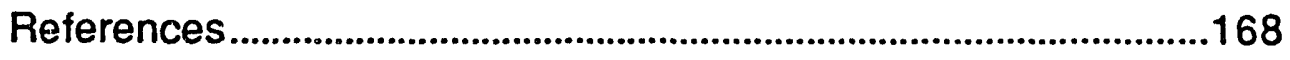

Appendix A: Major properties of EL2 in GaAs

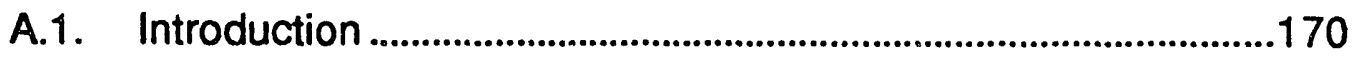

A.2. Deep level defects................................................................170

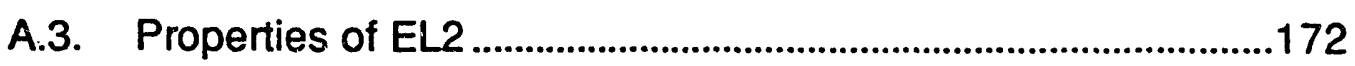

3.1. Electronic energy levels and the $\mathrm{CC}$ diagram................................................................................172

3.2. Energy levels determined by Hall effect and DLTS

3.3. Energy levels determined by optical

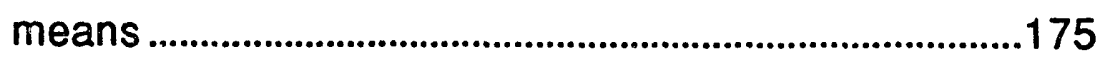

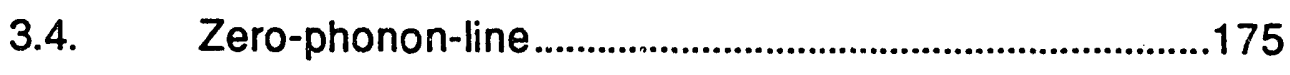

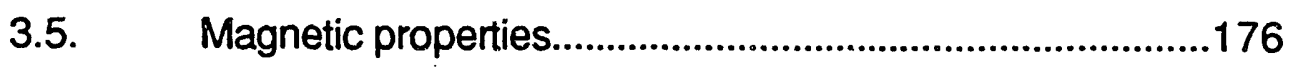

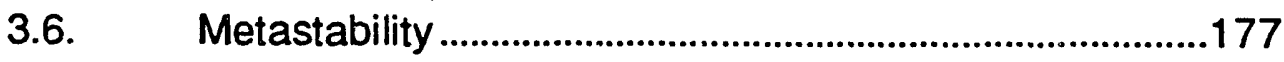

3.7. Microscopic models of EL2 structure .............................178

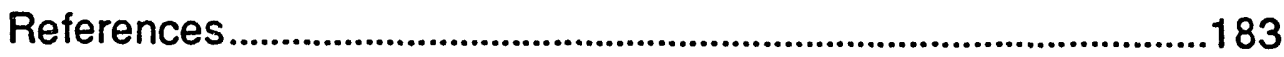

Appendix B: Solution of diffusion to a finite sphere ............................186

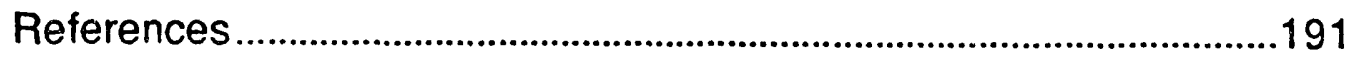


Being in a Materials Science and Engineering department of an academic institution, the emphasis of my work has been more on science than engineering. This is appropriate since the product of a university is educated individuals, not new technology. However, it is very difficult to separate technology from science. To say that science leads to new technology would be incorrect in most cases. More frequently the causality is actually reversed. New technology generally arises by trial and error or inductive means, a repeated observation. An inventor does not necessarily know why something will happen, only that it will happen. Science, on the other hand, is based in deductive reasoning. A hypothesis is formed. Its ramifications are logically deduced and then exhaustively tested experimentally (when possible). This process is ideally suited to the improvement of technologies rather than generating new technologies. It can be slow and cumbersome process though. Much like a bulldozer, it does not move quickly, but ultimately arrives at its destination.

Not surprisingly, it is the simple but elegant theory which is most robust and able to withstand the test of time. Similarly, it is the simple experiment that is most likely to unambiguously prove the theory. But what is it that stimulates human curiosity to form theories and test them? Often it is technology and the developement 
of tools to better the human condition. Although I have pointed the causality arrow from technology to science, it can be the scientific deduction, and especially the counter-intuitive one, that leads to a whole new field of technological endeavor. The marriage of science and technology could nave started with improved stone hand tools circa $10^{5}$ years ago, moving on to the bronze and iron ages, the developement of agriculture, the industrial age bringing in textiles, medicine, and now electronics and biotechnology. To divorce science from technology, would not be showing the whole picture. The elegant theory is often appealing when considered on its own but its application to people can be less abstract to comprehend. Therefore in the course of this dissertation, particularly in the motivation, technological application will be emphasized along side the scientific process. Hopefully the results of this work will be interesting enough to stand on their own as a piece of good scientific work. I also hope they are useful enough to serve as guidelines to a technologist fabricating devices made of lowtemperature-grown MBE GaAs. 


\section{Acknowledgements}

Sir Isaac Newton once said, $†$ "If I have seen further it is by standing on the shoulders of giants." In the course of my thesis research I have felt for the most part like I have been banging around amongst the ankles of giants, trying not to fall down. The support I have received from everyone in the group at LBL has given me the "hand up" I have needed to get my own view. I would first like to thank my advisor Eugene E. Haller. I have enjoyed his personal guidance and the broad range of experimental and theoretical capabilities he has made available to me within our laboratory and throughout the semiconductor science community. I especially appreciate the significant input of Wladek Walukiewicz. Theoretical discussions with him have been a most enjoyable apect of my studies.

Although a Ph.D. degree is a personal endeavor, many have been directly involved in this work. I would also like to thank Kam Chan for his keen interest and staying after hours to grow the GaAs material for this study. Other collaborators who have been directly involved in this research are, Joel Ager: Raman spectroscopy, Hans Bornemann: Magnetic susceptability measurements, Piotr Dreszer: EPR measurements, Noble Johnson: SIMS measurements, Paulo

\footnotetext{
† Letter to Robert Hooke, February 5, 1675/6.
} 
Santos: photocurrent spectroscopy, Shoichiro Tanigawa: positron annihilation measurements, In Chin $\mathrm{Wu}$ and Alain Claverie: TEM characterization.

I would also like to thank those outside the laboratory, the softball Native Defects, my cycling friends and the earth science crew with Monday night hoops for helping maintain my sanity. Finally, the understanding and encouragement of my parents, sisters and most of all Nancy is warmly appreciated. All of you have been a giant help to me.

This work was supported by the Director, Office of Energy Research, Office of Basic Energy Sciences, Materials Science Division of the U.S. Department of Energy under Contract DE-AC03$76 \mathrm{~F} 00098$

David E. Bliss November, 1992 


\section{Chapter 1. Overview of Semiconductor Technology and Review of LTMBE GaAs}

\subsection{Semiconductor Technology}

\subsubsection{An historical perspective}

Semiconductor technology can be considered the utilization of the unique electrical and optical properties of serniconductors. As a speculative example, the first application of a semiconductor might have been by an early human who marveled at the sparkle and brilliance of a diamond. The diamond appeared visually unique as compared to other rocks and so was chosen as an ornamental object. Without realizing it, the early human had selected the diamond because it had a large bandgap and large dielectric constant characteristic for a semiconductor. These materials parameters made the diamond transparent to visible light and highly refractive and therefore appealing to the eye.

This example might seem a little contrived, but it serves to illustrate that the use of semiconductors is older than might be expected. Certain properties of semiconductors have been known for well over a century. In 1833, Faraday measured the temperature dependence of the resistivity of conducting materials. He noted (Faraday 1839) a negative temperature coefficient of resistivity for salts of silver sulfide, contrary to that found for metals. This 
property is characteristic of the thermal excitation of free carriers across the bandgap of a semiconductor. It forms the basis of a temperature measuring semiconductor device known as the thermistor.

In 1873, Smith (Smith 1873) disovered that exposing selenium crystals to light could reduce their resistance. This property is characteristic of the optical excitation of free carriers across the bandgap of a semiconductor.

This discovery led to perhaps the first device application of a semiconductor recorded in the scientific literature. In 1880, Bell (Bell 1880) exploited the photoconductive properties of selenium to create his "photophone." The principle of Bell's photophone was to use a voice to modulate the intensity of a collimated beam of light. The light was then projected upon a selenium crystal. The electrical output of the selenium detection element was an exact replica of the initial voice. It is remarkable the similarity the photophone bears to the current technology of fiber-optic communication. It has all the same functional components as the current technology, a source (lamp instead of LED or laser), transmitting medium (air instead of glass fiber), and receiver (Se instead of some other semiconductor like A(GaAs), yet it predates the current fiber-optic communication by more than a century. Bell's invention was truly visionary.

In 1874, Braun (1874) discovered the rectifying nature of a metallic contact to semiconductor crystals of copper-, iron-, and lead sulfide. The metal-semiconductor point contact was applied early in this century as a rectifier in the first radio receivers. The semiconductor crystal was the "crystal" of a "crystal radio set." In 
the quest for more gain, however, vacuum tubes replaced the "crystal" for radio applications. The invention of radar during World War II necessitated receivers which could operate at frequencies higher than were attainable by vacuum tube rectifiers. Semiconductor detectors were the only devices capable of operating at radar frequencies. Today, yet higher frequencies are used for saitellite communications. Specially fabricated GaAs transistors form the front end transponders operating at up to $100 \mathrm{GHz}$.

The invention of the transistor in 1947 must be considered the cornerstone of the semiconductor revolution. Bardeen and Schockly's announcement of the transistor action of metallic point contacts on Ge was the spark that led to an explosion of research on semiconductor materials. It was apparent that the transistor would replace its vacuum tube counterpart for most applications. The small size meant inherent space saving and robustness. The lack of a heated filament meant much lower power requirements. Radios could be portable. Military electronic equipment could be made smaller, lighter and stronger. The semiconductor device had arrived as a major technological influence upon our lives.

A second semiconductor revolution would occur in the 1960's with the advent of device integration. Fabricating many transistors in the same piece of $\mathrm{Si}$ allowed a build-up of functionality unobtainable with discreet devices. Si integration is the basis of the microprocessor and computer industry we have today. The commercial success of the computer industry has fueled the unprecedented research effort on semiconductor materials. Besides the advancement of semiconductor device technoiogy, a main resuit 
of this effort has been a gain in the understanding of the properties of solids and the development of the trasic field of solid state physics. Specifically, theories were developed and tested regarding the effective mass representation of band extrema and shallow impurity states, electronic transport, optical and thermal properties of semiconductors.

\subsubsection{Devices, a materials perspective}

As one moves from the first discovery of certain semiconductor properties to the present day "high-tech" applications, the most important work occurred during the last half century in the understanding and development of semiconductor materials. The build up of an empirical, theoretical, and technical knowledge base is so enormous and detailed that it is beyond the scope of this introduction and could itself be the subject of several volumes of books. However, it is possible to elaborate on a common theme throughout the development of semiconductor materials for device applications. That theme is the manipulation of the carriers of electric charge within a semiconductor.

The operation of a semiconductor device can be considered to be the control of the flow of free carriers, electrons and holes, under the influence of some stimulus. The result is a change in the output of the device. For example, the stimulus could be an applied voltage or current, illumination by light, a change in temperature, the presence of a magnetic field, etc. The output could be a change in voltage, current, or the emission of light. Although this set of variables seems small, the variety of possible devices is essentially infinite. The variety occurs because different applications require 
different magnitudes of input stimulus, different magnitudes of output response, different functional dependence of the output on the input, and different time response.

Take for example the transistor. It probably covers the widest range of variation for a single device type. The transistor is essentially a current gain device. Power transistors need to output large currents, several amperes for the output stage of a stereo to drive speakers. A high impedance preamplifier might require extremely small leakage currents to amplify a sensitive detector with an output of a few electrons per second. Satellite communication requires high frequencies, maybe $30 \mathrm{GHz}$, while a dc measurement system requires stability. Examples are limitless.

A device's function is basically determined by distribution of charge within it. The important parameters are

1.) the free carrier concentration,

2.) the spatial distribution of free carriers,

3.) the propagation time of carriers across the device,

4.) the carrier lifetime.

An engineer designing a device controls this set of parameters to achieve the desired function. The methods available for the control of these parameters have become increasingly sophisticated with the advancement of semiconductor materials.

The general development of semiconductor materials has progressed from obtaining high purity elemental semiconductors, to binary compound semiconductors, to alloyed ternary and quaternary compounds, to heterostructure combinations of alloyed semiconductors. During this time the methods to purify starting 
material, grow good quality crystals, and precisely incorporate dopants into very small structures have dramatically improved.

At first, semiconductors were plagued by uncontrollably high concentrations of impurities and defects. Irreproducible results and devices were the norm. The background concentration of free carriers was too high. The first stage of the semiconductor technology was the development of high purity elemental crystals of $\mathrm{Ge}$ and $\mathrm{Si}$. Chemical means were found to form volatile compounds of $\mathrm{GeCl}_{4}$ and $\mathrm{SiCl}_{4}$ which could be fractionally distilled to parts per billion impurity levels. A further means to purify elemental semiconductors was invented by Pfann (1955). It involved repeatedly passing a molten zone along the length of a solid $\mathrm{Ge}$ bar taking advantage of the solubility difference of impurities in the solid versus the liquid. Ge crystals can now be grown with residual impurities controlled down to a level of less than 1 impurity in $10^{12}$ host atoms ( $<4 \times 10^{10} \mathrm{~cm}^{-3}$ net impurities).

Only with a pure starting material as a basis was it possible to control the deliberate reintroduction of free carriers. Elements from the columns of the periodic table neighboring column IV which contains diamond, $\mathrm{Si}$ and $\mathrm{Ge}$, were intentionally added as dopants. The methods to introduce dopants advanced from metallic alloying with limited control over the spatial distribution and concentration, to solid state diffusion and ion implantation. $P-n$ junctions could be formed with tailored doping profiles to create diodes for a wide range of applications. With $p-n$ junctions as the building block many new devices were created by stacking junctions together. Back to back diodes created the $p-n-p$ structure which is the transistor. 
Adding another junction created a $p-n-p-n$ thyristor. For integrated circuits millions of junctions were fabricated side by side. Some served not only in the active region of individual devices, but also as a means to isolate neighboring transistors.

In parallel to the work on elemental semiconductors, researchers began exploring the realm of compound semiconductors. Group III elements could be combined with group V, group II with group VI to create zinc-blende structure semiconductors. Welker discovered InSb and other III-V's by melting the constituents together on his stove at home.

The compound semiconductor crystals lacked the inversion symmetry of $\mathrm{Si}$, and contained an ionic component to the bonding which was no longer purely covalent. This had the effect of altering the band structure in some compound semiconductors so that holes and electrons could recombine directly leading to the emission of light. Compound semiconductors, like InAs or GaAs, with a smaller effective mass than $\mathrm{Si}$ gave rise to higher mobility carriers. More efficient devices which operate at higher frequencies and lower power consumption could be fabricated

Compound semiconductors are not limited to binary alloys. Ternary or quaternary alloys which contain three or four elements respectively, can be grown for a continuously adjustable range of semiconductor properties. For example, by adjusting the $\mathrm{Ai}$ content $x$, of an $A l_{x} \mathrm{Ga}_{1-x} A s$ alloy, the bandgap at $300 \mathrm{~K}$ can be adjusted from $1.43 \mathrm{eV}$ to $1.65 \mathrm{eV}(0.75$ to $0.87 \mu \mathrm{m})$ as $x$ varies from 0 to 0.3 . Thus, the wavelength of emitted photons from $A l_{x} \mathrm{Ga}_{1-x} A s$ LED's can be compositionally tuned. Figure 1.1 shows the optical and near ir 


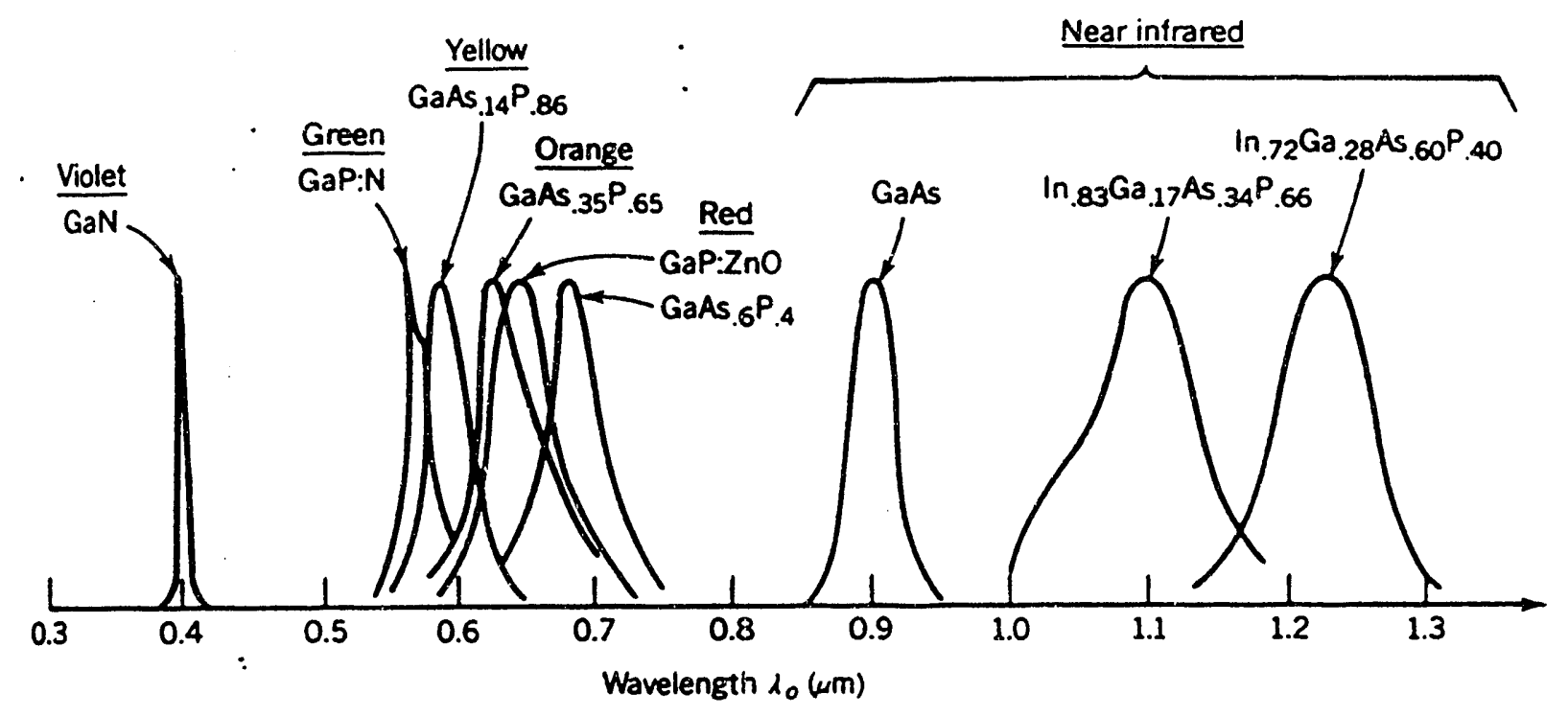

FIG. 1.1 Spectral density of emitted light versus wavelength for semiconductor LED's. Peak intensities have been normalized to the same value. (Adapted from (Sze 1981))

emission bands for a variety of compound semiconductors illustrating the wide range of wavelengths which can be covered. It should be mentioned that an important quaternary system for fiber optic communications is $\ln _{x} G_{a_{1-x}} A s_{y} P_{1-y}$. The tunable emission range $(1.1-1.6 \mu \mathrm{m})$ is well matched to silica optical fibers which have zero dispersion (propagation velocity is independent of wavelength) at $\lambda=1.3 \mu \mathrm{m}$ and a minimum attenuation at $\lambda=1.55 \mu \mathrm{m}$. Thus with the same semiconductor and optical fiber materials, one can take advantage of low dispersion for high bandwidth, high-speed communications or one can take advantage of the low attenuation for long distance communication. 


\subsubsection{Hetero-epitaxy}

Probably the single most important feature of compound semiconductor technology, in contrast to silicon technclogy, is the ability to combine different III-V alloys into a single-crystalline structure. Such structures are called hetero-structures. The prototypical example of a heterostructure device is the double heterojunction laser. It achieved the first continuous wave (cw) laser action in a semiconductor. This device is now found commonly in peoples pockets, contained in their compact disk player. The laser is used to probe the optical information contained on the disk. Applications of heterostructures are not just limited to lasers. Heterostructure bipolar transistors (HBT's) are expected to belong to the fastest future semiconductor devices. Other novel devices including the avalanche photodiode (APD) and high electron mobility transistor (HEMT) are based on heterostructures.

The application of heterojunctions in devices is often referred to as "Bandgap Engineering." The central idea behind bandgap engineering is that bandgap of the semiconductor is no longer fixed, but becomes a spatially variable design parameter. Offsets in the conduction and valence band edges can be used as barriers to confine carriers. Graded heterojunctions in HBT's permit the independent control of the distribution and flow of electrons and holes. This level of control allows for fabrication of devices unobtainable in straight $\mathrm{Si}$ or GaAs systems. Many working on this topic believe that the future of compound semiconductor devices lies in the realm of heterostructure devices and not GaAs metal-semiconductorfield-effect transistors (MESFET's). Why pay 10-100 times more for 
a GaAs MESFET based integrated circuit (IC) which only has slightly better speed and power advantages over it's Si IC counterpart? Compound semiconductor growth and device companies are banking on the future of unique heterostructure devices for burgeoning satellite communication applications and high definition television (HDTV).

The importance of heterostructures is not limited to devices. They have proved instrumental as probes of quantum effects. The confinement of a two dimensional electron gas at a $\mathrm{Si}_{-} \mathrm{SiO}_{2}$ heterojunction led to the discovery of the integer quantum Hall effect by von Klitzing in 1980 (1980). The Nobel prize was awarded for this discovery in 1985. Artificial structures, such as quantum dots and lines are being fabricated to test theories.

The ability to grow structures of such crystalline perfection and control at the atomic scale is a true wonder of human achievement. Several epitaxial growth techniques have been developed for the fabrication of heterostructures. They include liquid phase epitaxy (LPE), vapor phase epitaxy (VPE), and molecular beam epitaxy (MBE). Numerous variations of these three basic epitaxial growth techniques have been developed. The use of different starting gasses, called precursors, for VPE has been developed to take advantage of different growth chemistry. Chloride VPE (CIVPE) makes use of $\mathrm{AsCl}_{3}$ and related chloride compounds. Hydride VPE (HVPE) uses $\mathrm{AsH}_{3}$ and related hydride compounds. Organometallic VPE (OMVPE, MOVPE, or MOCVD depending in which research institute the growth occurred) employs $\left(\mathrm{CH}_{3}\right)_{3} \mathrm{Ga}_{3}$ and related methyl, ethyl or other organic based metallic compound 


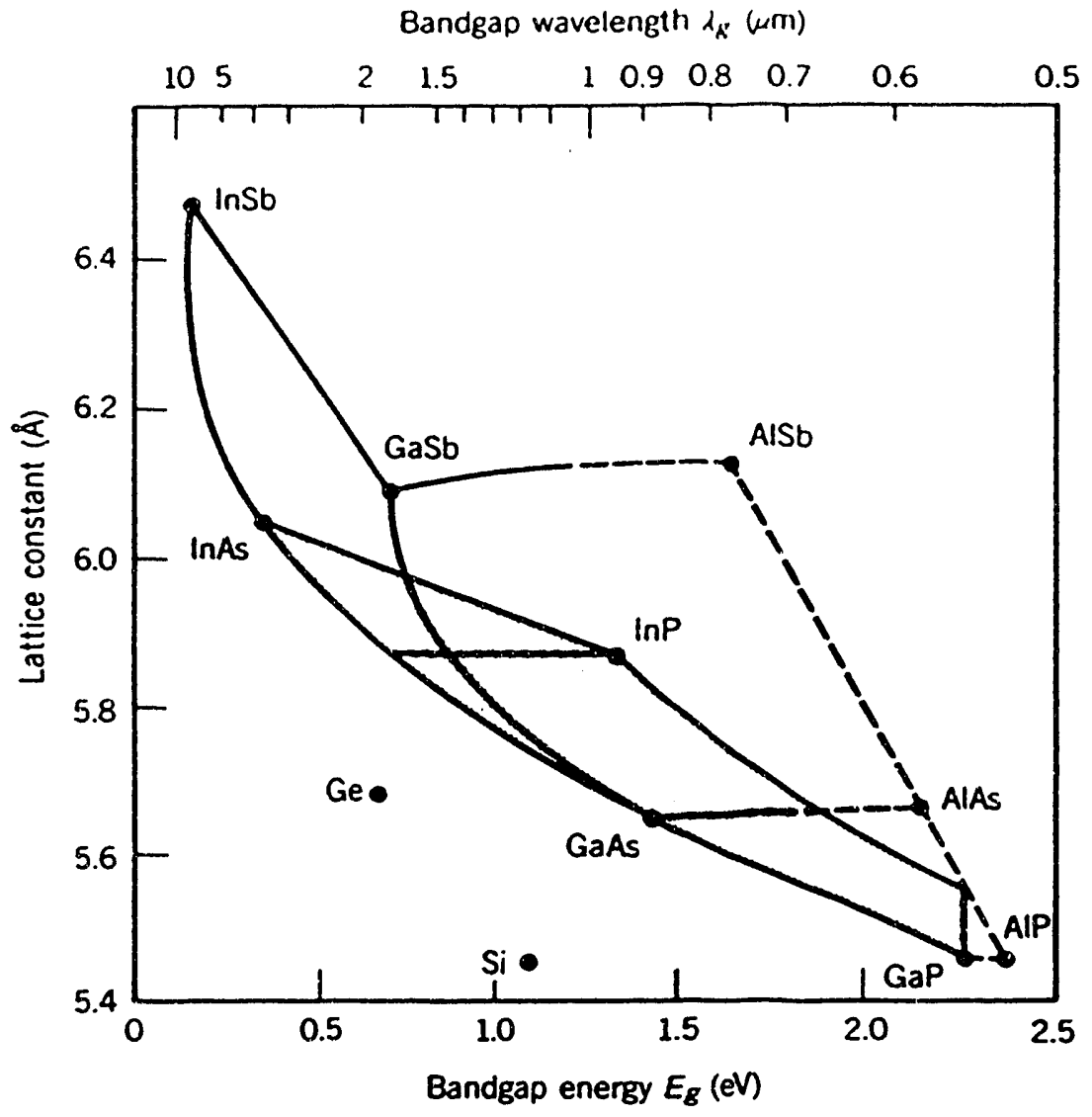

FIG. 1.2 Lattice constant vs bandgap energies or wavelengths for $\mathrm{Si}, \mathrm{Ge}$, and several III-V binary compounds are shown as points $(\bullet)$. Values for ternary compounds are represented by lines connecting the two constituent binary compounds. Solid and dashed lines represent direct and indirect bandgaps respectively. Quaternary alloys fall in the shaded area bounded by ternary compound lines, and binary compound vertices. The quaternary alloy $\ln _{1}$ ${ }_{x} G a_{x} A s_{2-y} P_{y}$ is represented by the cross hatched area bounded by the InAs, GaAs, GaP, and InP vertices (Saleh and Teich 1991).

precursor. A related variation of the MBE technique is metallorganic MBE (MOMBE) in which gas instead of solid sources are used. A wide range of growth techniques is available. Choice of the proper technique depends on the ability to grow the desired compound, spatial control of compositional and doping changes, required flexibility of growth conditions, throughput, toxicity, and cost. 
The best heterostructures are obtained when the epitaxial layers are grown on closely lattice matched substrates. Otherwise, in lattice mismatched systems dislocation relax the strain caused by the mismatch. A great deal of effort is currently spent to overcome these problem defects and grow GaAs on Si (Nozaki, et al. 1990, Uen and Sakawa 1991). Satisfactory results are yet to be obtained. Figure 1.2 shows the handgap vs. lattice constant for several III-V systems. It can be considered to be a crystal growers road map. The two most important systems involve the horizontal lines at the lattice constant of GaAs and InP since these are the two best bulk grown substrates. The ternary $A l_{x} G_{a_{1-x} A s}$ system is lattice matched to GaAs. The quaternary $\ln _{x} G a_{1-x} A s_{y} P_{1-y}$ sytem is lattice matched to $\ln \mathrm{P}$ substrates.

\subsubsection{Defects and Devices}

A crystal can be regarded as a regular array of atoms forming a repetitive pattern. More precisely, in an idealized "perfect" crystal, all the positions of atoms can be described by the translation of a unit cell by an integral number of vectors defining the unit cell. However, to be thermodynamically stable a real crystal must contain point defects. Point defects can be divided into two categories, native and extrinsic defects. Native defects are an intrinsic flaw in the host crystal, with an atom in the wrong place (not described by a unit cell translation) or missing. An extrinsic defect is the inclusion of an impurity atom in the crystal. Similar elements from neighboring columns of the periodic table typically occupy substitutional sites forming shallow electronic levels near the band edges. In contrast, dissimilar elements such as 
transition metals occupy sites interstitially between host atoms and form deep electronic levels in the middle of the bandgap.

In an elemental semiconductor the principle native defects are the vacancy, where the atom is missing, and the interstitial where the atom occupies a site not described by the unit cell translation. In a compound semiconductor, the number of possible defects increases dramatically. Two possible vacancies exist, $V_{I I I}$ and $V_{V}$, on the group III and $\mathrm{V}$ sites respectively. At least two possible interstitials exist, $\mathrm{III}_{\mathrm{i}}$ and $\mathrm{V}_{\mathrm{i}}$, and more if the different interstitial sites are considered. Also, for compound semiconductors there is the new possibility to form antisite defects which cannot occur in elemental semiconductors. An antisite defect consists of an atom occupying the wrong sublattice, ie., a group III element occupies a group $\mathrm{V}$ site, IIIV, and vice versa, $\mathrm{V}_{\mathrm{III}}$. The number of possible native defects increases even further for ternary and quaternary alloys with more possible permutations to put an atom on the wrong site. Note, this is considering only isolated point defects. The possibility of defect complexes increases the number of defect configurations factorially.

Defects in semiconducting materials play a critical role in determining the material properties. Even a small concentration of defects can drastically alter material characteristics from the "perfect" crystalline state. Free carrier mobility and lifetime are strongly affected by the presence of defects, greatly changing optical and transport properties.

It would be incorrect, though, to make the general statement that all defects degrade device performance and lower fabrication 
yield. In actuality, many devices would not function without the presence of defects. The term "Defect Engineering" has been coined to describe the controlled introduction of native defects. Instead of trying to eliminate all defects, the goal of defect engineering is to introduce beneficial kinds of defect at specific locations and concentrations within the crystal analogous to the reintroduction of impurities as dopants to create devices.

One of the first broad technological applications of using impurities other than shallow dopants in semiconductors, dates back to early Si technology. Au (a deep level impurity) was introduced as a recombination center in $\mathrm{Si}$ diodes to increase switching rates by reducing minority carrier lifetimes. This might not exactly be considered Defect Engineering, since $A u$ is not a native defect, but the same principles apply. A more appropriate application of defect engineering is the comrnon practice to damage the backside of $\mathrm{Si}$ wafers producing large concentrations of structural defects which in turn getter transition metal impurities.

Defect engineering is being used to help realize the light emitting and carrier mobility advantages of GaAs over Si. In GaAs, AsGa-related antisite defects play a fundamental role in creating intrinsic-like insulating material. EL2, a defect associated with AsGa (and perhaps identical to $A s_{G a}$ ), is responsible for compensating shallow impurities and pinning the Fermi level at midgap to create semi-insulating (SI) GaAs (Holmes, et al. 1982, Ta, et al. 1982). Wafers of SI GaAs are the highly resistive substrates used to fabricate integrated circuits.

\subsubsection{LTMBE Buffer Layers}


An even more defective, but still useful, GaAs material is grown by molecular beam epitaxy (MBE) at low substrate temperatures (LTMBE). More than a decade ago it was proposed (Muratani, et al. 1978) that GaAs grown at low temperatures would make an excellent buffer layer for field effect transistors. This material contains defect concentrations three orders of magnitude greater than bulk SI GaAs and is exploited as a buffer layer between the SI substrate and the active device layer.

GaAs metal semiconductor field effect transistors (MESFETs) are typically fabricated on SI substrates. The active areas of the device are created by ion implantation into the substrate or by epitaxial deposition. Several problems with the MESFET performance are due to the SI substrate. These problems are backgating, hysteresis of the drain-source current $\left(I_{d s}\right)$ depending on the drain-source voltage, low source-drain breakdown voltage and short channel effects (Barrera 1975, Eastman and Shur 1979). Backgating is the most serious effect, degrading both analog and digital circuit performance.

Backgating can be defined as the drain-source current in the channel of one device being influenced by voltage applied to the gate of a neighboring device. Figure 1.3 shows a schematic cross section of a typical MESFET structure fabricated on SI GaAs illustrating the space charge regions (Chen, et al. 1989). For a typical MESFET, Ids of the n-type channel is reduced by a negative potential applied to a neighboring device or the substrate. A space charge region is formed at the interface between the active channel layer and the substrate. Backgating results since the substrate conducts a small but 


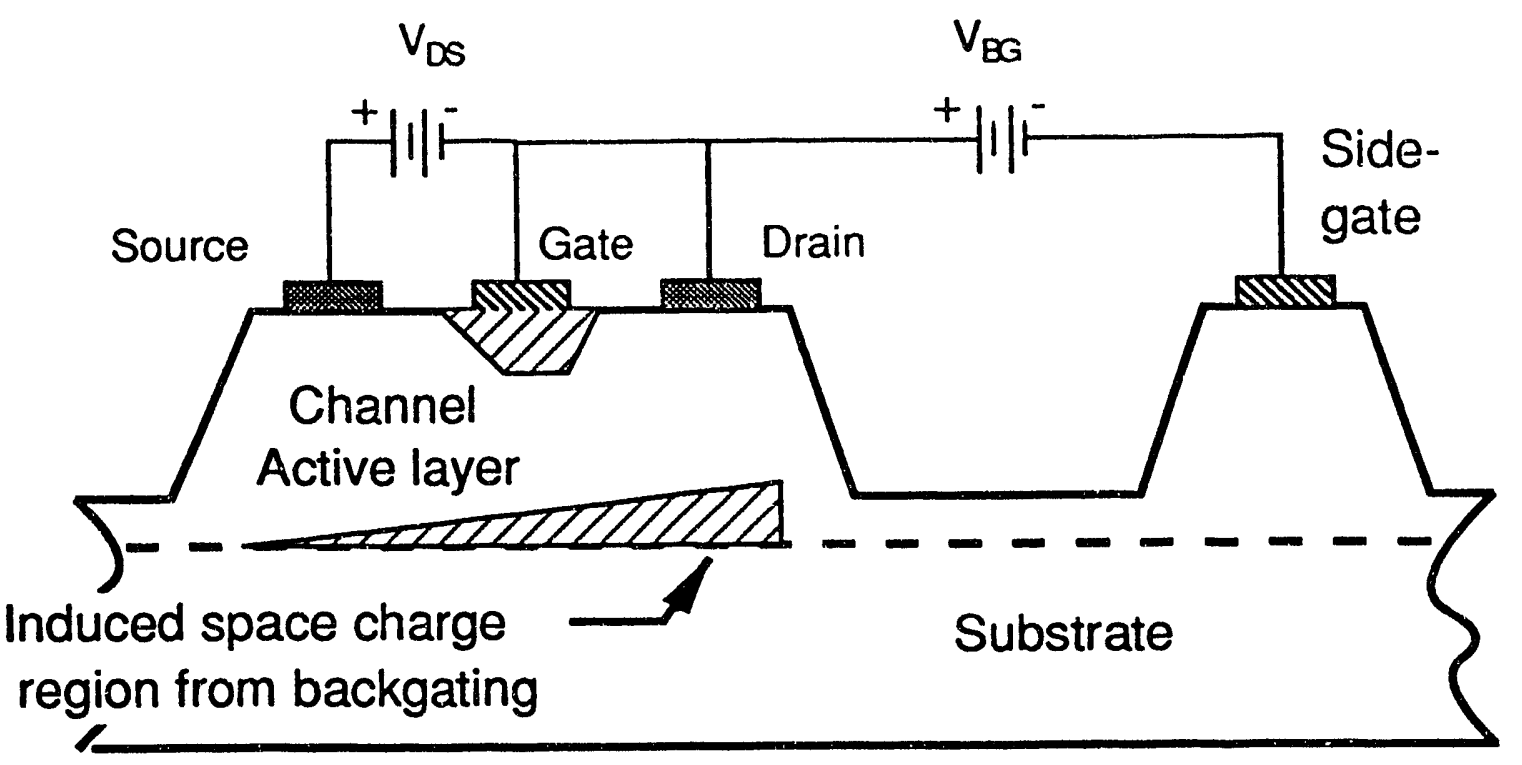

FIG. 1.3 Schematic cross section of a GaAs MESFET plus sidegate test structure. $V_{D S}$ is the applied drain source voltage generating a drain source current IDS. $V_{B G}$ is the applied backgate or sidegate voltage. The space charge region where backgating occurs.is shown, illustrating the undesirable pinch off of the channel.

sufficient current to change the space charge region and pinch off the channel, reducing Ids.

To reduce backgating a more resistive buffer layer is often inserted between the substrate and active layer. Several different layers have been proposed ranging from undoped GaAs, AlGaAs heterostructures and superlattices (Schaff, et al. 1984), to ion implantation damage isolation (Short and Pearton 1988). The success of these buffer layers in reducing backgating has been only marginal, until the recent development of GaAs buffers grown by MBE at low substrate temperatures, $200-300^{\circ} \mathrm{C}$. The LT MBE buffer layers have completely eliminated backgating (Smith, et al. 1987). Figure 1.4 shows the results of this backgating study, plotting lds versus the sidegate voltage for a variety of buffer material in light 


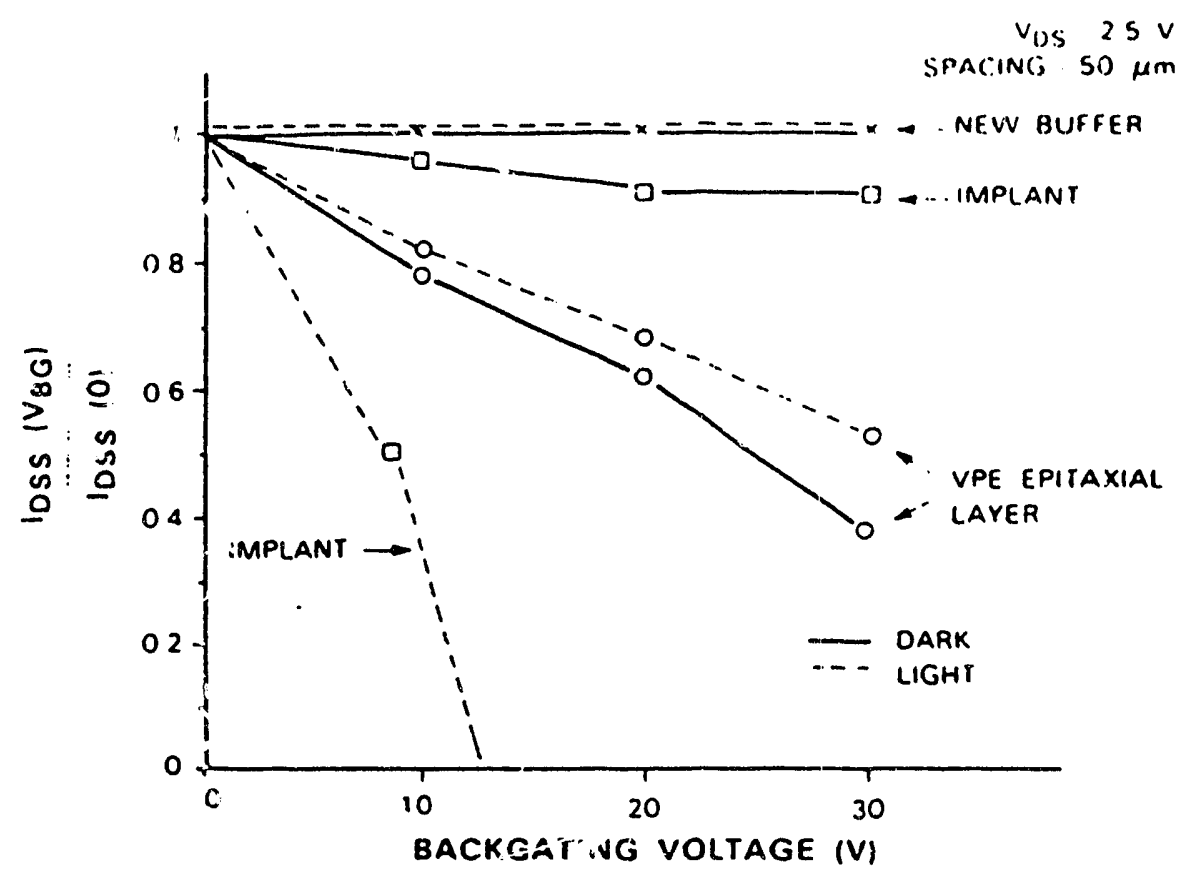

(a)

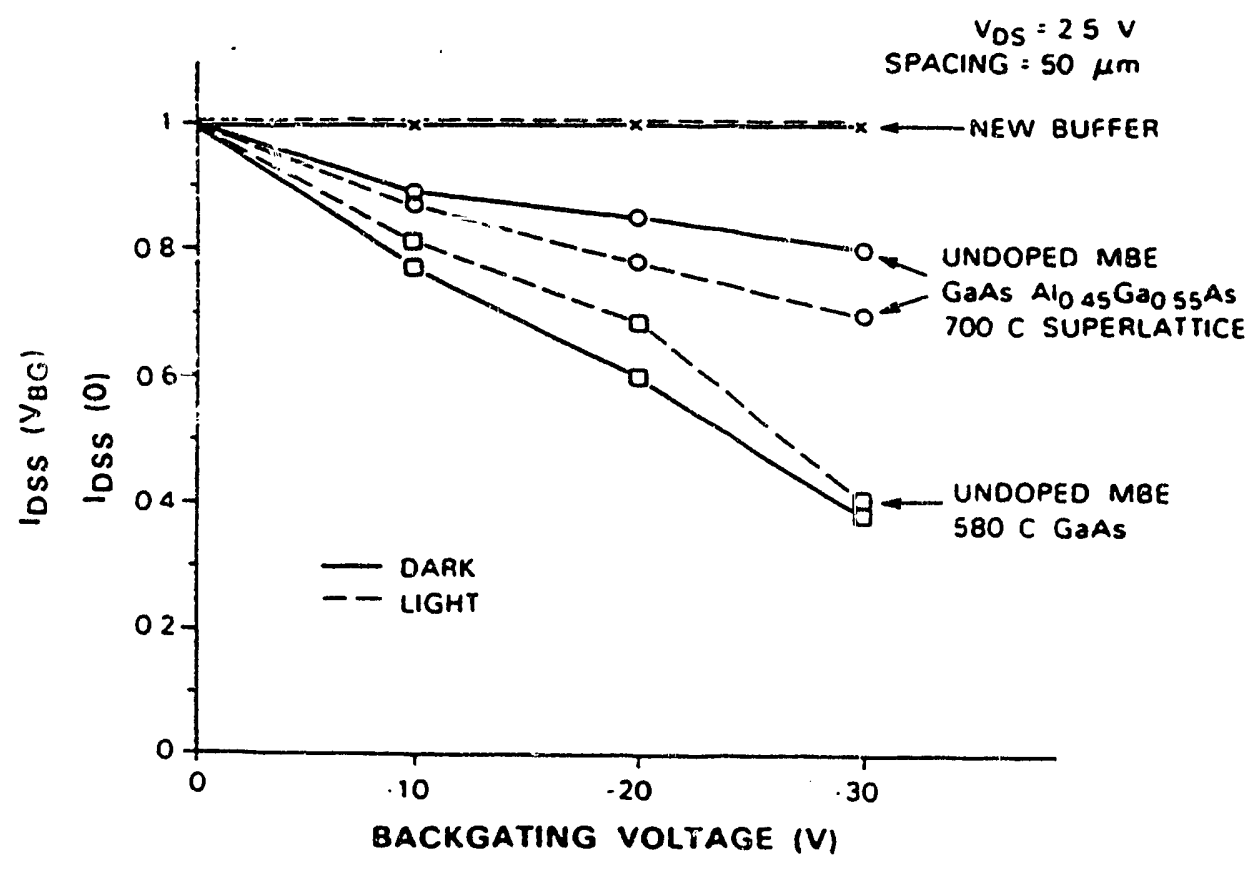

(b)

FIG. 1.4 Plot of MESFET Ids vs. sidegate voltage for a variety of GaAs materials. The solid lines indicate results obtained in the dark and dashed lines in the light. Note the elimination of sidegating effects when a LTMBE buffer layer is used between ihe substrate and active layer (Smith, et al. 1987). 
and dark conditions. For the LT MBE buffer layers, no reduction in $I_{d s}$ occurs for gate voltages up to $30 \mathrm{~V}$ in comparison to an ion implanted MESFET in SI GaAs which shows a $10 \%$ reduction. The elimination of backgating in GaAs integrated circuits by using LTMBE buffer layers is a significant technological achievement. Neighboring transistors can be fabricated at $1 / 3$ the distance resulting in a 10 fold increase in integration density.

\subsection{The MBE Technique}

Growth of materials by the Molecular-Beam Epitaxy (MBE) technique (Herman and Sitter 1988) is basically the deposition of a material by evaporation under ultra high vacuum (UHV) conditions. Fig. 1.5 shows a simplified schematic diagram of an MBE system. The main feature of the system is the large UHV chamber which can be pumped down to a base pressure of 10-10 torr. The walls of the chamber and hot sources are shrouded with a $L_{2} N_{2}$ cooled shield to limit contamination from the walls. Within the chamber are the substrate holder and heater, the various elemental sources, and substrate transfer mechanisms. The sources are resistively heated crucibles. The rate of evaporation is determined by the vapor pressure of the material which is determined by the source temperature. The sources can be mechanically shuttered to turn on or off the beam of neutral atoms or molecules. The evaporated atoms or molecules travel across the chamber with a thermalkinetic energy below $1 \mathrm{eV}$, eventually arriving at the substrate. The substrate is usually rotated to insure uniform coverage. The substrate is heated to enhance the surface diffusion so that the 


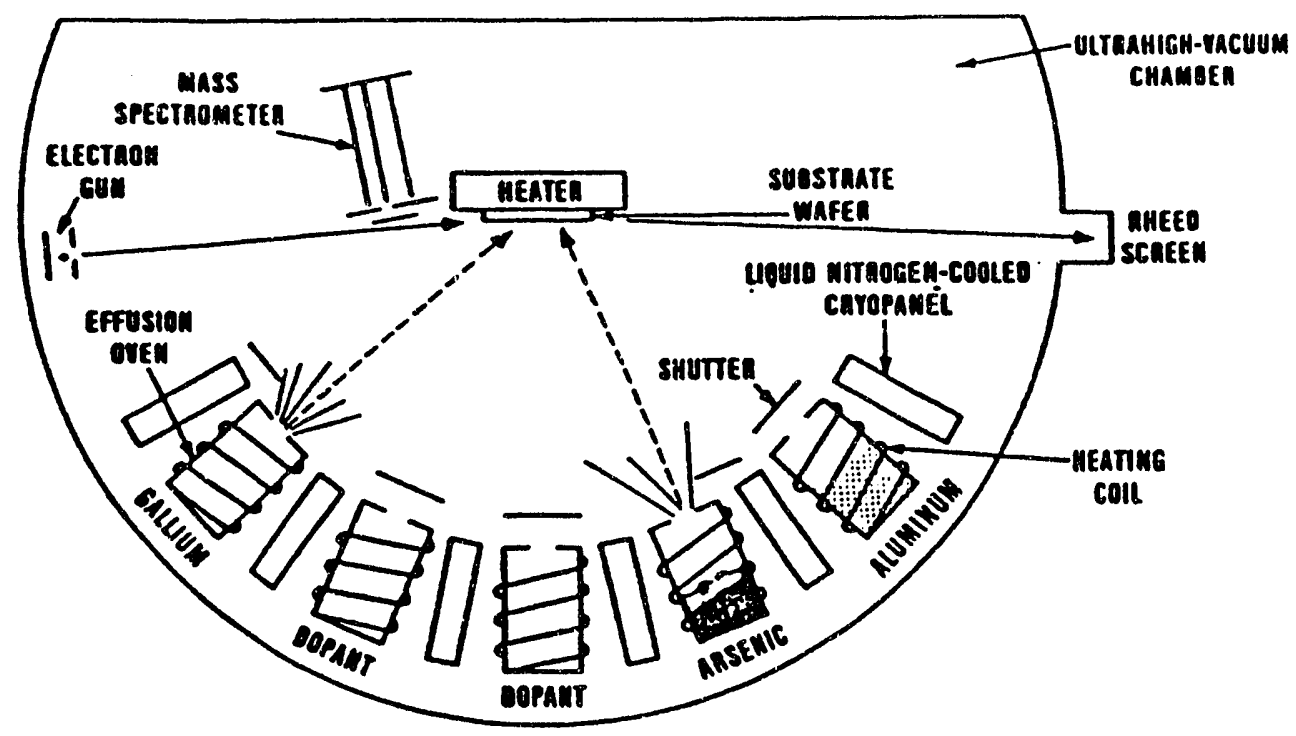

XBL. $8310-11946$

FIG. 1.5 Simplified schematic of a typical MBE system for GaAs growth.

arriving atoms can find a proper site for crystallization. The growth rate is low, on the order of $1 \mu \mathrm{m} / \mathrm{h}$ or $1 \mathrm{monolayer} / \mathrm{s}$, to ensure the migration of surface atoms to proper sites before being covered by arriving atoms and to allow layer by layer growth.

Since the growth is occurring in UHV conditions, various in situ electron beam techniques are used to characterize the growing layers. Reflection High Energy Electron Diffraction (RHEED) and Auger electron spectroscopy are the more commonly employed ebeam techniques. Optical techniques such as ellipsometry and photoreflectence difference spectroscopy (Colas, et al. 1991) are also used to monitor the growth. 
The ability to grow high quality GaAs by MBE is due to the unique properties of As. The sticking characteristics of As at the surface is most important. As arrives at the surface as an As4 tetramer, which is a tetrahedron with an As at each vertex. The tetramer will only break up and be incorporated into the film if $\mathrm{Ga}$ is present at the surface to which the As can bond. If the surface is completely passivated by As, the tetramer does not stick. This property of As makes it possible to grow stoichiometric GaAs under a wide range of conditions.

The advantages of MBE over other epitaxial growth techniques are several. The material has a high purity with background impurity concentrations in the $10^{14} \mathrm{~cm}^{-3}$ range. Atomically abrupt doping and compositional interfaces can be fabricated which are crucial for device applications. The MBE technique is flexible, regarding the composition of materials and layer sequences. Some problems exist with $P$ based compounds.. $N$-ny ternary and quaternary alloys can be grown as layers which can not be grown in bulk because they do not melt congruently and would phase separate.

\subsection{Growth of LTMBE GaAs}

The growth of the LTMBE GaAs buffer layers does not differ considerably from that of conventional MBE GaAs. The primary difference is the substrate temperature which ranges from 200$300^{\circ} \mathrm{C}$ as compared to approximately $600^{\circ} \mathrm{C}$ for conventional MBE GaAs. Like conventional MBE GaAs the new buffer is grown using Ga and $\mathrm{As}_{4}$ beams under an excess As flux which stabilizes the surface and a $\mathrm{Ga}$ flux which limits the growth rate to $1 \mu \mathrm{m} / \mathrm{h}$. The beam- 
equivalent-pressure (BEP) ratio of the $A s / G a$ beam is around 10 . The BEP is determined by inserting an ion gauge in the path of the molecular beam and measuring the pressure. This corresponds to a beam flux ratio, As $4 / G a$, of approximately 2 .

\subsection{Properties of LTMBE GaAs}

In this section, published structural, electrical and optical properties of LTMBE GaAs will be reviewed. Particular attention will be focussed on how the properties of LTMBE layers differ from standard MBE GaAs.

\subsubsection{Structural properties}

The structural properties LTMBE GaAs layers have been explored both during and after the growth by a variety of techniques. During growth at $200^{\circ} \mathrm{C}$, the observed reflected high energy electron diffraction (RHEED) patterns (Smith, et al. 1988a) reveal planar growth of single crystals. High resolution transmission electron microscopy (TEM) images and $x$-ray diffraction patterns (Kaminska, et al. 1989) confirm that good quality single crystals can be grown.

The $x$-ray experiments indicate that a considerable amount of strain is grown into the layers. This is intriguing considering the growth is homo-epitaxial, i.e., GaAs layers on a GaAs substrate. Usually strain in epitaxial layers is associated with lattice mismatch of hetero-epitaxial systems like InGaAsP on GaAs. Double crystal $x$-ray diffraction studies of unannealed layers show a $0.1 \%$ increase in lattice parameter for layers grown at $200^{\circ} \mathrm{C}$, but no increase for layers grown at $300^{\circ} \mathrm{C}$ (Kaminska, et al. 1989). Another study of layers grown between $180-210^{\circ} \mathrm{C}$ shows that the amount of 
TABLE 1.1 Dependence of the structural properties of LTMBE GaAs layers on the substrate growth temperature. Lattice expansion was measured by doublecrystal $x$-ray diffraction, critical thickness by TEM and excess As concentration by PIXE. No change to polycrystallinity observed in $2.7 \mu \mathrm{m}$ thick samples ( $\mathrm{Yu}$ and Liliental-Weber 1991).

\begin{tabular}{cccc}
\hline $\begin{array}{c}\text { Growth } \\
\begin{array}{c}\text { Temperature } \\
\left({ }^{\circ} \mathrm{C}\right)\end{array}\end{array}$ & $\begin{array}{c}\text { Lattice } \\
\text { expansion } \\
(\Delta \mathrm{a} / \mathrm{a} \%)\end{array}$ & $\begin{array}{c}\text { Critical } \\
\text { Thickness } \\
(\mu \mathrm{m})\end{array}$ & $\begin{array}{c}\text { Excess As } \\
\left(\mathrm{N}_{\mathrm{As}}-\mathrm{N}_{\mathrm{Ga}}\right) / 2 \mathrm{~N} \\
(\text { at. \%) }\end{array}$ \\
\hline 180 & 0.15 & 0.3 & 1.23 \\
190 & 0.13 & 1.6 & 0.74 \\
200 & 0.10 & $>2.7^{*}$ & 0.35 \\
210 & 0.097 & $>2.7^{*}$ & 0.25 \\
\hline \hline
\end{tabular}

lattice expansion depends strongly on the growth temperature ( $\mathrm{Yu}$ and Liliental-Weber 1991). The results of that study are summarized in Table 1.1. The lattice expansion increases as the growth temperature is lowered.

Although single crystalline layers can be grown at substrate temperatures below $200^{\circ} \mathrm{C}$, a critical thickness exists above which the crystallinity breaks down (Yu and Liliental-Weber 1991). See Table 1.1 for values of the critical thickness. Pyramidal defects begin to form rendering subsequently deposited material polycrystalline. The thickness at which the the layers become polycrystalline is reproducible for a particular growth temperature.

The mechanism responsible for layers becoming polycrystalline has not yet been determined. One suggestion is increasing surface roughness during the growth nucleates misoriented GaAs grains. Another interesting suggestion is strain induced dislocation formation. Similar to hetero-epitaxial systems, LTMBE GaAs grows pseudomorphically, i.e., the lattice constant of the growing layer is forced to that of the substrate. A tetragonal 
distortion occurs to conserve the specific volume and strain energy is accumulated within the layer. At a certain thickness the energy required to form a strain relieving misfit dislocation becomes less than the build up of strain energy and dislocations are formed at the surface. However, because the growth temperature is so low the dislocation cannot glide to the interface to relieve the strain. As a result, dislocations pile up at the surface nucleating misoriented growth. Another suggestion is that increasing surface roughness during the course of the growth eventually nucleates randomly oriented crystals.

It has been suggested that the critical thickness be used as a tool to calibrate the substrate temperature of MBE systems in this low temperature range (Calawa 1990). Accurately monitoring the substrate temperature during growth can be difficult. In the research MBE chamber at Lincoln Laboratory, substrates are mounted with indium to the heater giving an accurate temperature measurement. However, in a typical commercial MBE system the substrate is not mounted with indium and the temperature is measured with a thermocouple near the substrate. Since the substrate is not in intimate contact with the heater or the thermocouple, system to system temperature variations can be on the order of $50^{\circ} \mathrm{C}$. However, run to run variations in the same system are on the order of $5^{\circ} \mathrm{C}$. This makes it possible to calibrate the temperature via the critical thickness to the temperature of the Lincoln Laboratory machine. Tight temperature control is essential in order to compare results from layers grown in different MBE systems. Material properties are extremely temperature dependent. 
Substrate temperatures determined by this fashion have been sometimes referred to as degrees Calawa.

\subsubsection{Excess As}

The most significant feature of LTMBE GaAs, from which all the unique properties of the material are derived, is the presence of an extremely high concentration of excess As. Excess As is defined by the ratio $\left(N_{A s}-N_{G a}\right) /\left(N_{A s}+N_{G a}\right)$ where $N_{A s}$ and $N_{G a}$ are the number of $\mathrm{As}$ and $\mathrm{Ga}$ atoms per unit volume. Auger electron spectroscopy (AES) (Smith, et al. 1988b) and proton induced $x$-ray emission (PIXE) (Kaminska, et al. 1989) studies indicate $\sim 1-2$ at. \% excess As for material grown at $200^{\circ} \mathrm{C}$. Figure 1.6. shows the equilibrium phase diagram for GaAs. The existence region for solid
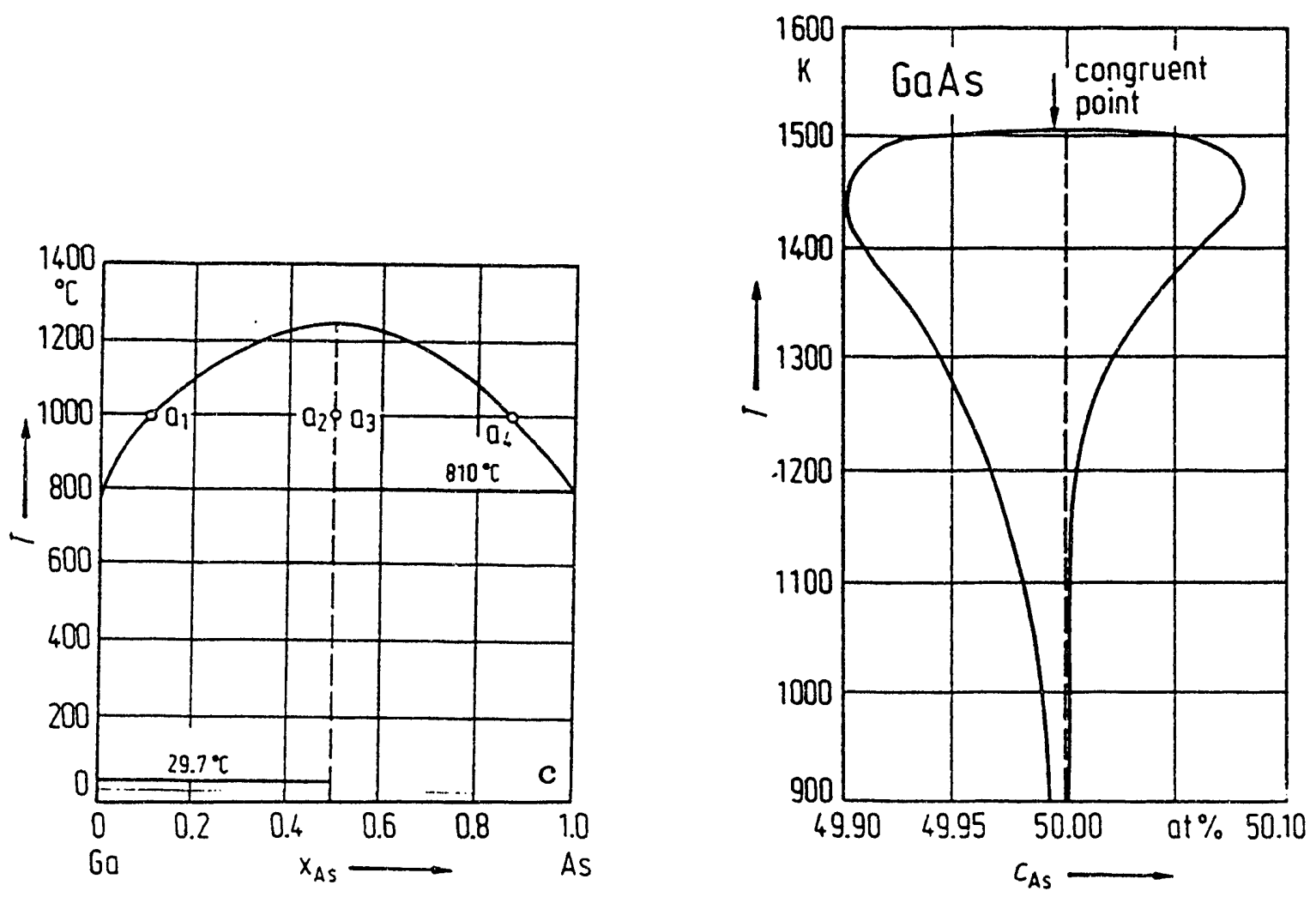

FIG. 1.6. Phase diagram for GaAs. The existence region for solid GaAs is expanded to show detail. 
GaAs is extremely narrow and has been expanded. Typical values for the maximum deviation from stoichiometry for bulk grown GaAs is $0.05 \%$ (Terashima, et al. 1986) or nearly 2 orders of magnitude less than for LTMBE GaAs. In the phase diagram of Fig. 1.6, LTMBE GaAs would lie about 1 meter to the right of the page.

In a study by Liliental et al. (1991) the dependence of the excess As concentration on growth temperature was investigated. Those results are summarized in Table 1.1. The amount of excess As increases as the growth temperature is lowered from 210 to $180^{\circ} \mathrm{C}$. Above $300^{\circ} \mathrm{C}$ no excess As can be detected (Kaminska, et al. 1989).

The reason for the incorporation of excess As at low growth temperatures can be explained intuitively. For standard MBE GaAs grown at $580^{\circ} \mathrm{C}$, an arriving As tetramer is thermally desorbed from an As passivated surface, if there is no $\mathrm{Ga}$ atom with which to bond. If there is an available $\mathrm{Ga}$ site, the As tetramer is broken up forming GaAs. As the growth temperature is lowered, the thermal energy becomes insufficient to desorb an unbonded As tetramer, generating an As rich layer.

\subsubsection{Native point defects}

How is the excess As contained in the LTMBE layers as grown? TEM micrographs show the material is single crystalline with some small precipitates (Liliental-Weber, et al. 1991). However, the amount of As contained in the precipitates is not sufficient to account for all the excess As as measured by PIXE. Most of the excess As exists in the form of point defects.

IR absorption spectroscopy of LTMBE GaAs layers reveals a broad absorption band at a wavelength of $1.2 \mathrm{eV}$ (Manasreh, et al. 
1990). A similar absorption band is seen for the EL2 defect in bulk LEC GaAs crystals (see Appendix A for a review of the properties of EL2). Like EL2, this absorption band can be photoquenched by white light illumination at low temperatures (Photoquenching will be covered in detail in the next section). However, unlike EL2 the absorption lacks a zero-phonon line $(Z P L)$ at $1.04 \mathrm{eV}$. Therefore, the defect observed in LTMBE GaAs is not assigned directly to EL2 but rather is referred to as EL2-like or AsGa-related. Because of the similarity of this defect to EL2 and the fact that most deep level defects in GaAs have an optical cross section of approximately $10^{-16} \mathrm{~cm}^{2}$, researchers have felt justified using the calibration determined by Martin for the optical absorption cross section of EL2० (Martin 1981). The concentration of neutral EL2-like defects in LTMBE has been determined to be on the order of $10^{19}-10^{20} \mathrm{~cm}^{-3}$ depending on the growth temperature and conditions.

\subsubsection{Metastability of AsGa-related defects}

A fraction of the ir absorption at $\lambda=1 \mu \mathrm{m}(1.2 \mathrm{eV})$ in LTMBE GaAs can be photoquenched (also referred to as photobleaching) by illumination with $1 \mathrm{eV}$ photons at low temperature, $\mathrm{T}<77 \mathrm{~K}$ (Manasreh, et al. 1990). The thermal recovery of the photobleached metastable state occurs when the sample is warmed above $140 \mathrm{~K}$ similar to EL2. For layers grown at $200^{\circ} \mathrm{C}$, the fraction of photoquenchable (PQ) absorption is about $10 \%$ of the total absorption at $1.2 \mathrm{eV}$.

Metastability of deep level defects has been observed in a wide range of compound semiconductors (Lang, et al. 1978, Wolk, et al. 1992). In general, metastability has been interpreted in terms of a 
large lattice relaxation (LLR) of the defect (Lang, et al. 1978). A LLR is the rearrangement of the atom(s) forming a defect when the charge state is changed or an electronic excitation occurs. A "large" relaxation would be an atomic displacement on the order of $1 \AA$. Metastability occurs because an energetic barrier exists for the atoms to return to the equilibrium position. A simple mechanical analog to LLR metastability is a hinge with an overcenter spring shown in Fig. 1.7 There are two locally stable positions for the hinge, (a) and (b). Position (b) is the metastabie position since the overcenter spring is more extended than for position (a). To move the hinge from (b) to (a) the energy barrier of further extending the overcenter spring before it can contract, must be overcome. The position of the hinge represents the position of atoms in the defect system. The springs represent the binding forces of valence electrons. In a typical defect system the energy barrier is a fraction of an $\mathrm{eV}$. Excitation to the metastable state is usually done optically at low temperature. Relaxation back to the stable configuration is accomplished by warming the sample.

In a well known paper (Chadi and Chang 1988), Chadi and Chang proposed a model for EL2 in which the metastability involved a LLR of an isolated AsGa. Since the concentration of AsGa-related defects in LTMBE GaAs which show metastability is so high, novel experiments exploring the effects of a large number of defects undergoing a LLR have been undertaken.

One experiment exploring the effects of LLR in LTMBE GaAs layers was performed by Khachaturyan (1992). The influence of the LLR of defects on the elastic properties of GaAs was 


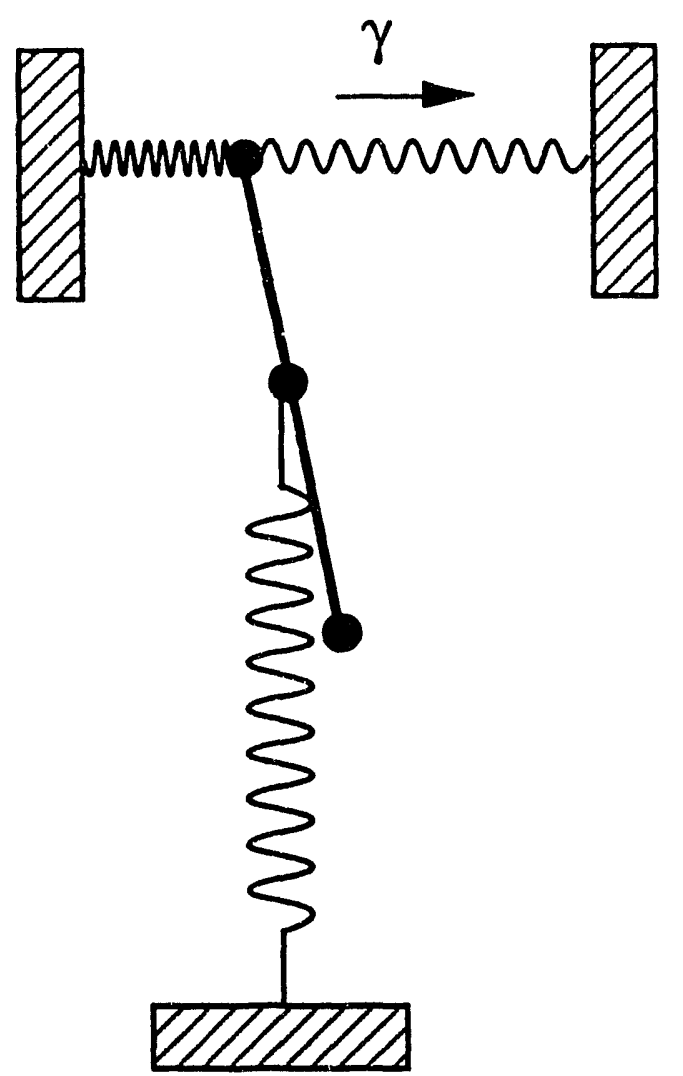

(a)

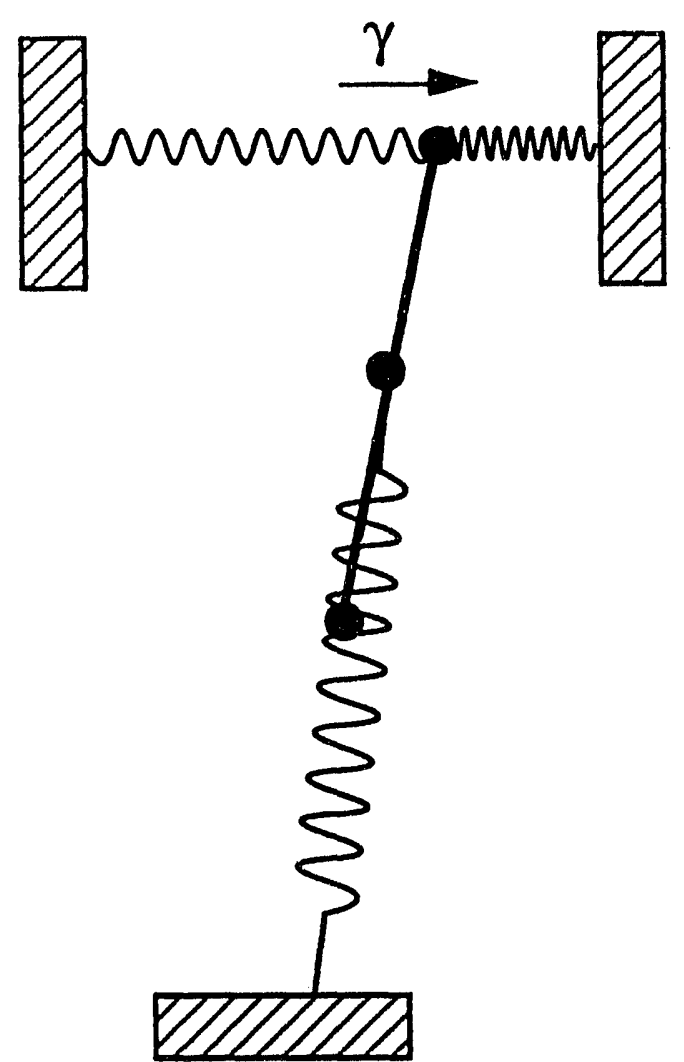

(b)

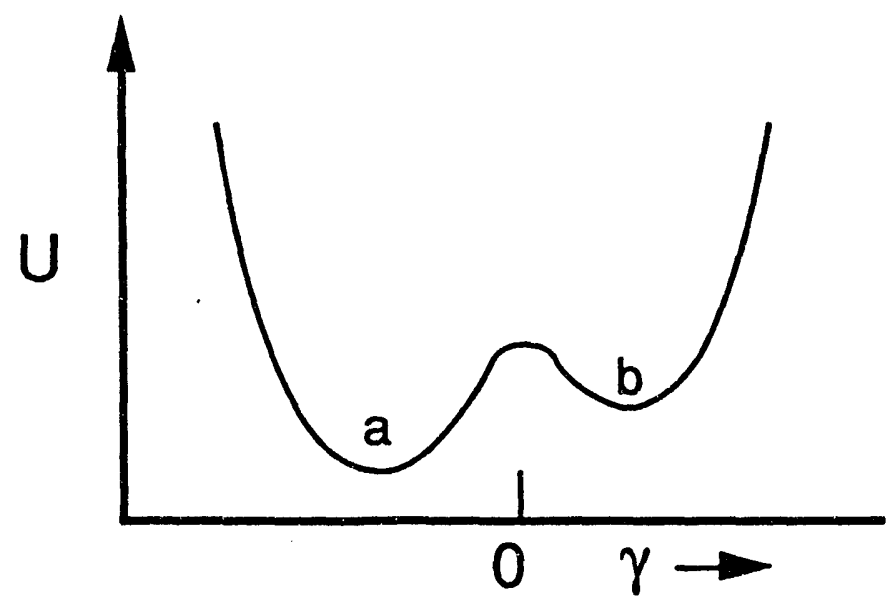

(c)

FIG.17. Mechanical analog to metastability: Schematic of a hinge with an overcenter spring. (a) equilibrium position. (b) metastable position. (c) configurational coordinate diagram of system energy vs. hinge position, $\gamma$. 
determined by measuring the propagation velocity of surface acoustic waves (SAW's) before and after photoquenching of the EL2like defects. A persistent increase of the SAW velocity occurred after illumination at low temperatures. The initial velocity could be recovered after warming the sample to $140^{\circ} \mathrm{C}$. This is experimental proof that a LLR is responsible for the metastability of defects in LTMBE GaAs.

In a related experiment, the $X$-ray diffraction peak from an LTMBE layer was measured at low temperature before and after photoquenching EL2-like defects (Kowalski and Leszczynski 1991). A decrease in the lattice parameter was found, which could be reversibly recovered by warming to $140^{\circ} \mathrm{C}$.

\subsubsection{Electrical properties}

Electron paramagnetic resonance (EPR) measurements performed on LTMBE GaAs lavers (Kaminska, et al. 1989) have shown the well known quadruplet signal characteristic of AsGa defects (Wagner, et al. 1980). The quadruplet is only seen in material grown below $200^{\circ} \mathrm{C}$ and even then, it is only visible in some samples. The $\mathrm{g}$ factor, $g=2.04$ and hyperfine interaction parameter, $A=0.09$, are the same as identified with singly ionized $\mathrm{AsGa}^{+}$defects in bulk GaAs. Therefore the observed defect in LTMBE GaAs is associated with an AsGa with four As ligands. A concentration of paramagnetic spins of $5 \times 10^{18} \mathrm{~cm}^{-3}$ was found in $200^{\circ} \mathrm{C}$ grown layers. The existence of $\mathrm{AsGa}^{+}$implies that some unknown acceptor must be compensating the AsGa.

The Fermi level for LTMBE GaAs is pinned at midgap as established by the presence of AsGa-related defects in both the 
neutral and singly ionized charge states. As such, one would expect SI material with an intrinsic value for the resistivity on the order of $\rho \cong 10^{7} \Omega \mathrm{cm}$ at $300 \mathrm{~K}$. However, as-grown LTMBE GaAs is quite conductive with $\rho$ in the range $10-10^{3} \Omega \mathrm{cm}$. The measured mobility is very low, $\mu<1 \mathrm{~cm}^{2} \mathrm{~V}^{-1} \mathrm{~s}^{-1}$. The low mobility suggests the conductivity mechanism is due to carriers hopping between the deep EL2-like defects (Kaminska and Weber 1990, Look, et al. 1990).

Hopping conductivity is the motion of localized carriers from an occupied defect or impurity center to a nearby empty center. The hopping probability is proportional to the electron wave function overlap and the concentration of empty sites (which is given by compensating minority centers). Wave functions drop off exponentially with distance so that hopping can be observed at a concentration just below the metallic transition, which is before the defects form a conduction band of their own. Generally, the hopping phenomenon in semiconductors is associated with shallow levels with large Bohr orbits, on the order of $100 \AA$. In the case of Ge shallow levels, hopping can be seen at temperatures of $10 \mathrm{~K}$ and below. The observation of hopping conduction near room temperature in LTMBE layers, though unusual, is not unique. Hopping conductivity has been observed in this temperature range in neutron irradiated GaAs (Coates and Mitchell 1972) and InP doped with Mn, a deep level transition metal impurity (Kuznetsov, et al. 1984). The difference from shallow level hopping is that it is occurring over deep states which are more localized, $r<10 \AA$, than shallow levels.

Variable temperature measurements of the resistivity of LTMBE layers show three regions of distinct behavior (Look, et al. 
1990). Above 350K, the conductivity is dominated by parallel conduction in the substrate and the electron excitation to the conduction band of the layer. Between 250-350K nearest neighbor hopping conductivity prevails and a linear Arrhenius temperature dependence is seen. Below 200K variable range hopping is the predominate mechanism. The temperature dependence of the resistivity obeys a T-1/4 law. Note however the temperatures delineating these regions are not very abrupt and can vary widely depending on the growth conditions.

The electrical measurements reported thus far, are that the resistivity increases as the substrate temperature during growth is lowered.

\subsubsection{Optical properties}

The IR absorption spectra show a broad absorption band around $1 \mu \mathrm{m}$ due to AsGa-related defects. Refer to section 1.4.3 discussing native defects in LTMBE GaAs for a descriptien of the ir absorption.

Photoluminescence (PL) optical characterization results of $L T$ MBE buffer layers have been reported in the literature (Smith, et al. 1988a). There is an order of magnitude decrease in the total photoluminescence intensity recorded at $5 \mathrm{~K}$ for layers grown at $300^{\circ} \mathrm{C}$ as compared to conventionally grown layers. The band gap PL decreases while deep level bands appear at 1.2, 1.3 and $1.5 \mu \mathrm{m}$. These broad bands have been attributed to various deep levels. The layers grown at $200^{\circ} \mathrm{C}$ are optically inactive, showing no $\mathrm{PL}$ signal.

The reduced PL signal in LTMBE GaAs is attributed to short minority carrier lifetimes due to the high concentration of nonradiative recombination centers. Room temperature lifetimes in 
LTMBE GaAs are on the order of 400 is (Gupta, et al. 1991), while for normal GaAs lifetimes are on the order of tens of nanoseconds. If one considers simple Schockley-Read-Hall recombination theory for the trapping mechanism at defects, then using a trap density of $\mathrm{N} \sim 1018 \mathrm{~cm}^{-3}$ (the material has been annealed), a capture cross section of $\sigma=10^{-13} \mathrm{~cm}^{2}$ (typical value for deep levels in GaAs), and a room temperature thermal velocity, $v_{\mathrm{th}}=1.6 \times 10^{7} \mathrm{~cm} \mathrm{~s}^{-1}$, the carrier lifetime $\tau=1 /\left(N \sigma v_{t h}\right)$ for annealed LTMBE GaAs is $\tau \equiv 0.6$ ps.

\subsubsection{Other applications}

As pointed out earlier, LTMBE GaAs is effective as a buffer layer isolating MESFET's in GaAs IC's. Buffer layer applications utilize the high resistivity of the material. Another type of application is based on utilizing the short lifetime of minority carriers in LTMBE GaAs to make fast photoconductive detectors (Smith, et al. 1989) and switches (Motet, et al. 1991). The operating principle of such a device is very simple. A voltage is applied across to contacts on a LTMBE layer. When a laser pulse illuminates the region between the contacts the voltage is shorted and a pulse is generated. The rise time of the pulse is limited by the propagation time across the gap, which is determined by the gap spacing and the carrier mobility. The decay time of the pulse is limited by the carrier lifetime. Therefore, to get the shortest possible pulses it is important to have material with good mobility and short lifetimes. Since LTMBE GaAs is single crystalline it has a higher mobility advantage over amorphous and ion damaged material used in the past. The extremely short lifetimes and high breakdown voltage ensure 
that high voltage $(\sim 500 \mathrm{~V})$ pulses can be switched in 1.5 ps. LTMBE GaAs photoconductive switches are the fastest reported to date.

\subsubsection{Annealing characteristics}

When using LTMBE GaAs as a buffer layer, the subsequent growth of a "normal" MBE GaAs active layer at $580^{\circ} \mathrm{C}$ anneals the underlying buffer layer. Considering that as-grown LTMBE GaAs is only slightly resistive, $\rho=10-10^{3} \Omega \mathrm{cm}$, and becomes $\mathrm{SI}$ after heat treatment above $500^{\circ} \mathrm{C}$, exposing the buffer layer to the higher growth temperature is fortuitous. Annealing is a critical step in achieving desired materials properties of LTMBE GaAs. In this section the published annealing characteristics of LTMBE GaAs will be reviewed.

Critical structural properties such as the lattice constant and excess As incorporation are affected by annealing. The $0.1 \%$ lattice expansion of as-grown layers is completely relaxed by a $2 \mathrm{~min}$. heat treatment at $600^{\circ} \mathrm{C}$ (Kaminska, et al. 1989). PIXE measurements indicate that the 1 at. $\%$ excess As is still present after annealing (Yu and Liliental-Weber 1991), which means no As is lost from the layer.. Instead, annealing alters the form in which $A s$ is incorporated. TEM studies reveal the formation of As precipitates 2-5 nm in diameter (Liliental-Weber, et al. 1991, Melloch, et al. 1990, Warren, et al. 1990) after annealing at $600^{\circ} \mathrm{C}$. The microstructure of these As precipitates has been extensively studied by Liliental-Weber et al. (1991). The smaller precipitates, 2-3 $\mathrm{nm}$ in diameter, are found to have cubic symmetry and to be coherent with GaAs lattice. A cubic phase of free solid As has never been observed. Therefore, the cubic phase of As in a LTMBE GaAs 
matrix is a result of lowering the surface energy to minimize the total energy of small precipitates. As the size of the precipitates increases over $4 \mathrm{~nm}$, the common hexagonal phase of $A s$ is observed exclusively. In the larger precipitates, even though the interface is incoherent, the surface contribution to the total energy is less important than the volume contribution so the more stable hexagonal phase of As forms.

One must be careful when quoting typical values for the size and density of precipitates (and other material properties too!) in LTMBE GaAs since they strongly depend on growth conditions and can vary widely. For GaAs grown at $200^{\circ} \mathrm{C}$ and annealed at $600^{\circ} \mathrm{C}$, the precipitate diameters generally fall in the range 20-100 $\AA$ and the concentration of precipitates ranges from $10^{16}-1017 \mathrm{~cm}^{-3}$ (Warren, et al. 1990).

The annealing characteristics of the AsGa-related defects have been studied by ir absorption (Manasreh, et al. 1990). In this study the photoquenchable (PQ) and non-photoquenchable (NPQ) portions of the ir absorption were monitored. It was observed that NPQ AsGarelated defects begin to anneal at $300^{\circ} \mathrm{C}$. In contrast, the EL2-like $P Q$ absorption was observed to increase slightly up to an annealing temperature of $450^{\circ} \mathrm{C}$. At $500^{\circ} \mathrm{C}$ the total $P Q$ and $N P Q$ absorption decreased sharply. Annealing of AsGa-related defects in LTMBE GaAs in this temperature range is very similar to that in irradiation damaged and plastically deformed GaAs (Haga, et al. 1988, Weber, et al. 1982). The fact that the annealing temperature is much lower than that for EL2 in LEC GaAs $\left(1100^{\circ} \mathrm{C}\right)$ has led to speculation that AsGa-related defects in LTMBE GaAs are different from EL2 
(Manasreh, et al. 1990). The lower temperature annealing of AsGarelated defects has been interpreted as vacancy assisted diffusion of AsGa (Bliss, et al. 1992) and will be considered in chapter 3.

Annealing away AsGa-related defects drastically increases the resistivity of LTMBE layers. The room temperature (300K) resistivity increases from $\sim 100 \Omega \mathrm{cm}$ to $>10^{7} \Omega \mathrm{cm}$. This increase can be understood in terms of elimination of the hopping conductivity of electrons over the localized defect levels. The probability of a hopping transition is proportional to the defect wavefunction overlap which decays exponentially with distance away from the defect. As the concentration of defects decreases due to annealing, the separation increases which reduces the hopping probability exponentially.

Another novel explanation for the extremely high resistivity of annealed LTMBE layers has been suggested by Warren et. al (1990). Their idea is that the SI properties of LTMBE GaAs are due to buried depletion regions around As precipitates. The origin of the depletion regions is the pinning of the Fermi level at the As precipitate interface. The As precipitate is treated as a metal which forms a Shottky diode with the GaAs matrix. If the concentration of precipitates is high enough, then the depletion regions will overlap to fully deplete the GaAs and render it semi-insulating.

The actual mechanism which makes LTMBE GaAs semiinsulating is currently under debate. Although the buried Shottky model is intuitively appealing, its applicability to LTMBE GaAs is questionable in my opinion. If the Fermi level in the GaAs matrix is pinned near midgap already, no depletion regions will be formed 
around the precipitates. Also, material of such high resistivity has been generated by irradiation damage and plastic deformation. These materials are stoichiometric and do not contain As precipitates. Why invoke a new model to explain highly resistive behavior which has been observed before in GaAs material and has been understood without a buried Shottky model?

\subsubsection{Motivation}

Because of the practical importance of LTMBE GaAs as a buffer layer, most investigations have been of undoped materials. It has been reported in a number of papers that low temperature growth of highly non-stoichiometric GaAs renders any intentionally introduced shallow impurities ineffective. Thus, it has been shown in an early study (Muratani, et al. 1978) that the efficiency of Si donors is abruptly reduced for growth temperatures lower than $450^{\circ} \mathrm{C}$. Also, a recent study of the dependence of Si donor doping efficiency on the stoichiometry of GaAs MBE layers shows that any significant deviation from stoichiometric composition towards arsenic rich material always leads to reduced activity of Si donors (Winer, et al. 1991). Much less attention has been paid to the effects of growth temperature and non-stoichiometry on the efficiency of p-type doping (Duhamel, et al. 1981).

The aim of the present study is to shed light on the properties of defects and defect-impurity reactions in doped LTMBE GaAs. The thermal stability of defects in LTMBE GaAs doped in a wide concentration range is investigated. The annealing conditions required to activate dopants in LTMBE GaAs is determined. A model 
has been proposed which describes annealing of AsGa-related defects via gallium vacancy assisted diffusion.

\section{References}

Barrera, J., Proc. IEEE/Cornell Conference on High Speed Semiconductor Devices and Circuits (Ithaca, NY, 1975) pp. 135.

Bell, A.G. "Bell's Photophone." Nature 22, 15 (1880).

Bliss, D.E., W. Walukiewicz, J.W. Ager III, E.E. Haller and K.T. Chan. "Annealing Studies of Low Temperature Grown GaAs:Be." J. Appl. Phys. 71, 1699 (1992).

Braun, F. "Uber die Stromleitung durch Schwefelmetalle." Ann. Phys. Chem. 153, 556 (1874).

Calawa, A.R., Workshop on Low Temperature GaAs Buffer Layers (San Francisco, CA, 1990).

Chadi, D.J. and K.J. Chang. "Metastability of the isolated arsenic-antisite defect in GaAs." Phys. Rev. Lett. 60, 2187 (1988).

Chen, C.L., F.W. Smith, A.R. Calawa, L.J. Mahoney and M.J. Manfra. "Reduction of sidegating in GaAs analog and digital circuits using a new buffer layer." IEEE Trans. Electron Devices 36, 1546 (1989).

Coates, R. and E.W.J. Mitchell. J. Phys. C5, 113 (1972).

Colas, E., D.E. Aspnes, R. Bhat and A.A. Studna. "Insitu monitoring of crystal growth by reflectance difference spectroscopy." J. Cryst. Growth 107, 47 (1991).

Duhamel, N., P. Henoc, F. Alexander and E.V.K. Rao. Appl. Phys. Lett. 39, 49 (1981).

Eastman, L.F. and M.S. Shur. IEEE Trans. Electon Devices ED26, 1359 (1979).

Faraday, M., "Experimental Researches in Electricity." B. Quaritch, Ed., (London, 1839), vol. 1.

Gupta, S., M.Y. Frankel, J.A. Valdmanis, J.F. Whitaker, G.A. Mourou, F.W. Smith and A.R. Calawa. "Subpicosecond Carrier Lifetimel in GaAs Grown by moleculat Beam Epitaxy at low Terperatures." Appl. Phys. Lett. 59, 3276 (1991). 
Haga, T., M. Suezawa and K. Sumino. "Defects with deep levels in GaAs induced by plastic deformation and electron irradiation." Jap. J. Appl. Phys. 27, 1929 (1988).

Herman, M.A. and H. Sitter, "Molecular Beam Epitaxy Fundamentals and Current Status." M.B. Panish, Ed., Springer Series in Materials Science (Springer-Verlag, New York, 1988), vol. 7.

Holmes, D.E., T.T. Chen, K.R. Elliott and C.G. Kirkpatrick. Appl. Phys. Lett. 40, 46 (1982).

Kaminska, M., Z. Liliental-Weber, E.R. Weber, T. George, J.B. Kortright, F.W. Smith, B.-Y. Tsaur and A.R. Calawa. "Structural properties of As-rich GaAs grown by molecular beam epitaxy at low temperatures." Appl Phys. Lett. 54,1881 (1989).

Kaminska, M. and E.R. Weber, "Hopping conduction of low temperature GaAs within an arsenic antisite defect band." E.M. Anastassakis and J.D. Joannopouloss, ed., 20 th International Conference on the Physics of Semiconductors (ICPS) (World Scientific, Thessaloniki, Greece, 1990) vol. 1, pp. 473.

Kaminska, M., E.R. Weber, Z. Liliental-Weber, R. Leon and Z.U. Rek. "Stoichiometry-related defects in GaAs grown by molecular-beam-epitaxy at low temperatures." J. Vac. Sci. Technol. B7, 710 (1989).

Khachaturyan, K., E. Weber and R. White. "Surface-Acoustic-Wave Study of Defects in GaAs Grown by Molecular-Beam Epitaxy at 220-Degrees-C." Phys. Rev. B45, 4258 (1992).

Kowalski, G. and M. Leszczynski, Materials Research Society (Boston, MA, 1991).

Kuznetsov, V.P., M.A. Messerer, E.M. Omelhanovsky and A.J. Polyakov, "The evidence for the Existance of the Hopping Conductivityvia Deep Impurity States: InP:Mn." J.D. Chadi and W.A. Harrisons, ed., $17^{\text {th }}$ international Conference on the Physics of Semiconductors (ICPS) (Springer Verlag, San Francisco, CA, USA, 1984) pp. 643.

Lang, D.V., R.A. Logan and M. Jaros. Phys. Rev. B 19, 1015 (1978).

Liliental-Weber, Z., A. Claverie, J. Washburn, F.W. Smith and A.R. Calawa. "Microstructure of Annealed Low-Temperature-Grown GaAs Layers." Appl. Phys. A 53, 141 (1991).

Look, D.C., D.C. Walters, M.O. Manasreh, J.R. Sizelove, S. C.E. and K.R. Evans. "Anomalous Hall-effect results in low-temperature molecular-beam-epitaxial GaAs: Hopping in a dense EL2-like band." Phys. Rev. B42, 3578 (1990). 
Manasreh, M.O., D.C. Look, K.R. Evans and I .E. Stutz. "Infrared absorption of deep defects in molecular-beam-epitaxial GaAs layers grown at $200^{\circ} \mathrm{C}$ : Observation of an EL2-like defect." Phys. Rev B. 41, 10272 (1990).

Martin, G.M. "Optical assessment of the main electron trap in bulk semiinsulating GaAs." Appl. Phys. Lett. 39, 747 (1981).

Melloch, M.R., N. Otsuka, J.M. Woodall and A.C. Warren. "Formation of arsenic precipitates in GaAs buffer layers grown by molecular beam epitaxy at low substrate temperatures." Appl. Phys. Lett. 57, 1531 (1990).

Motet, T., J. Neew, S. Williamson and G. Mourou. "1.4 ps rise-time high-voltage photoconductive switching." Appl. Fhys. Lett. 59, 1455 (1991).

Muratani, T., T. Shimanoe and S. Mitsui. "Growth Temperature Dependence in Molecular Beam Epitaxy of GaAs." J. Cryst. Growth 45, 302 (1978).

Nozaki, S., N. Noto, T. Egawa, A.T. Wu, T. Soga, T. Jimbo and M. Umeno. "Effect of growth temperature and V/III ratio on MOCVD-grown GaAs-on-Si." Jap. J. Appl. Phys. 29, 138 (1990).

Pfann, W.G. and K.M. Olsen. "Zone-Melting." Bell Syst. Tech. J. 33, 201 (1955).

Saleh, B.E.A. and M.C. Teich, "Fundamentals of Photonics." Wiley series in pure and applied optics (John Wiley and Sons, 1991).

Schaff, W.J., L.F. Eastman, B. Van Rees and B. Liles. J. Vac. Sci. Technol. B2, 265 (1984).

Short, K.T. and S.J. Pearton. J. Electrochem. Soc. 135, 2835 (1988).

Smith, F.W., A.R. Calawa, C.L. Chen, M.J. Manfra and L.J. Mahoney, $45^{\text {th }}$ Annual Device Research Conf. (Santa Barbara, CA, 1987).

Smith, F.W., A.R. Calawa, C.L. Chen, M.J. Manfra and L.J. Mahoney. "New MBE Buffer Used to Eliminate Backgating in GaAs MESFETs." IEEE Electron Device Lett. 9, 77 (1988a).

Smith, F.W., C.L. Chen, G.W. Turner, M.C. Finn, L.J. Mahoney, M.J. Manfra and A.R. Calawa, Presented at 1988 IEDM (1988b).

Smith, F.W., H.Q. Le, V. Diadiuk, M.A. Hollis, A.R. Calawa, S. Gupta, M.Y. Frankel, D.R. Dykaar, G.A. Mourou and T.Y. Hsiang. "Picosecond GaAsbased photoconductive Optoelectronic detectors." Appl. Phys. Lett. 54, 890 (1989).

Smith, W. "The Action of Light on Selenium." Journal of the Society of Telegraph Engineers 2, 21 (1873). 
Sze, "Physics of Semiconducting Devices." (Wiley Interscience, 1981).

Ta, L.B., H.M. Hobgood, A. Rohatgi and R.N. Thomas. J.Appl. Phys. 53, 5771 (1982).

Terashima, K., O. Ohmori, A. Okada, M. Watanabe and T. Nakanisi, "Lattice Parameter Variation Depending on Stoichiometry of Undoped LEC GaAs." (OHM North-Holland, 1986) pp. 187.

Uen, W.Y. and S. Sakawa. "Comparative study of amorphous and crystalline buffer layers in MBE growth of GaAs on Si." J. Cryst. Growth 115, 122 (1991).

von Klitzing, K., G. Dorda and M. Pepper. Phys. Rev. Lett. 45, 494 (1980).

Wagner, R.J., J.J. Krebs, G.H. Strauss and A.M. White. Solid State Commun. 36,15 (1980).

Warren, A.C., J.M. Woodall, J.L. Freeouf, D. Grischkowskky, D.T. . Mcinturff, M.R. Melloch and N. Otsuka. "Arsenic precipitates and the semi-insulating properties of $\mathrm{GaAs}$ buffer layers grown by low-temperature molecular beam epitaxy." Appl. Phys. Lett. 57, 1331 (1990).

Weber, E.R., H. Ennen, U. Kaufmann, J. Windscheif, J. Schneider and T. Wosinski. "Identification of $\mathrm{As}_{\mathrm{Ga}}$ antisites in plastically deformed GaAs." J. Appl. Phys. 53, 6140 (1982).

Winer, K., M. Kawashima and Y. Horikoshi. Appl. Phys. Lett. 58, 2818 (1991).

Wolk, J., W. Walukiewicz and E.E. Haller. "Discovery of a New DX Center in InP Under Large Hydrostatic Pressure." (submitted to Phys. Rev. Lett. 1992).

Yu, K.M. and Z. Liliental-Weber. "Lattice site locations of excess arsenic atoms in gallium arsenide grown by low-temperature molecular beam epitaxy." Appl. Phys. Lett. 59, 3267 (1991). 


\section{Chapter 2. Experimental Results: As-grown LTMBE GaAs}

This chapter covers the results of the structural, electrical and optical characterization of as-giown LTMBE GaAs layers. In addition to the investigation of undoped material, the characterization of layers intentionally doped with Be acceptors and Si donors is reported. First, a brief review of the characterization technique is given with the results on undoped material. Then the results of measurements on doped LTMBE layers are presented and interpreted. Many of the characterization techniques used are the same as reported in the literature which was reviewed in the introduction. The present results are critically compared to those already reported.

The two main aspects of studying doped material were first to understand the compensation mechanism producing highly resistive material and second to determine what influence the Fermi level position or impurities have on the formation of defects.

\subsection{Growth Conditions:}

Samples were grown in a Varian Gen II MBE system by Dr. Kam Chan at the Hewlett-Packard Co. Microwave Technology Division, Santa Rosa, CA. The substrates were (100) oriented SI LEC GaAs wafers. The substrate temperature was monitored by a thermocouple in proximity to the substrate heater. The growth cycle 
consisted of two steps. First $100 \AA$ of conventional MBE GaAs was grown at $580^{\circ} \mathrm{C}$. Then the temperature was ramped down $300^{\circ} \mathrm{C}$ to grow a $3 \mu \mathrm{m}$ thick layer of LTMBE GaAs. The surface was As stabilized with an $\mathrm{As}_{4} / \mathrm{Ga}$ beam equivalent pressure ratio of 10 . The growth rate for the layers was $1 \mu \mathrm{m} \mathrm{hr}^{-1}$. Be and Si concentrations of $10^{16}, 10^{17}, 10^{18}$, and $10^{19} \mathrm{~cm}^{-3}$ were introduced in a series of samples.

\subsection{Characterization of undoped LTMBE GaAs}

\subsubsection{Structural Properties}

The significant structural properties of LTMBE layers to characterize relate to the crystallinity, stoichiometry, lattice constant, point defects and As precipitation. To get answers about these structural issues, a variety of characterization techniques were employed. The stoichiometry was analyzed by particle induced $X$-ray emission (PIXE). X-ray diffraction was used to detarmine the crystallinity and the lattice constant of the layers. Transmission electron microscopy (TEM) addressed issues of crystallinity and the presence of As precipitates. AsGa point defects were investigated by IR absorption and electron paramagnetic resonance (EPR). Positron annihilation was used to identify the presence of $V_{G a}$. The results of these characterization techniques on undoped LTMBE layers follow.

PIXE

PIXE measurements were performed by $\mathrm{Dr}$. Kin Man $\mathrm{Yu}$ on the van de Graaff accelerator at Lawrence Berkeley Laboratory. A 1.2 


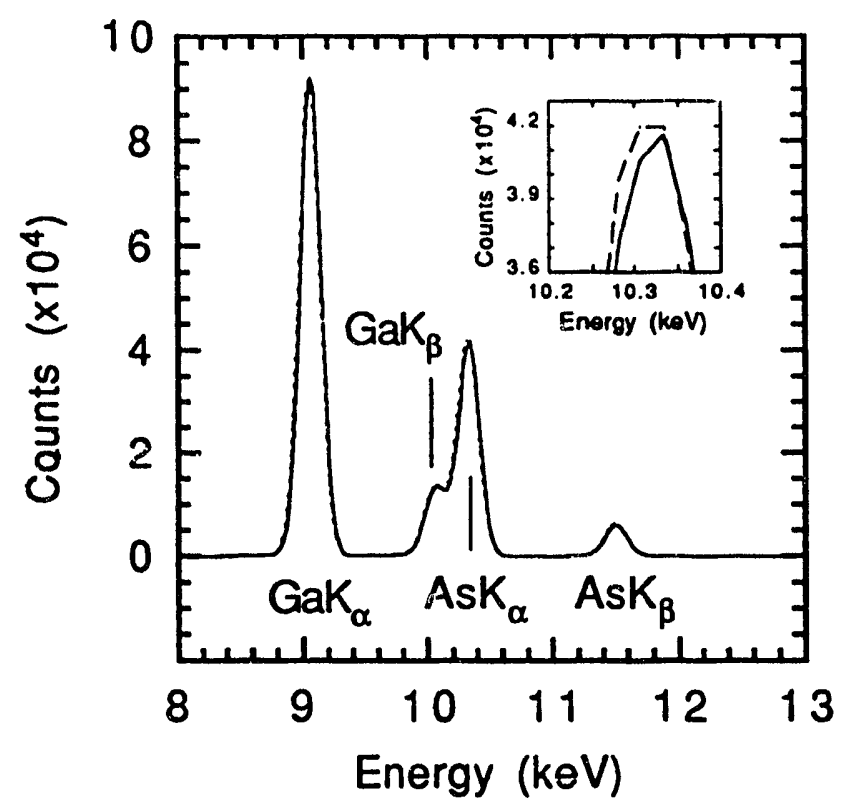

FIG. 2.1 Results of particle induced $x$-ray emission (PIXE) experiments: $x$ ray fluorescence spectra of undoped LEC GaAs (solid line) and LTMBE GaAs:Si $10^{19} \mathrm{~cm}^{-3}$ (dashed line). Inset shows the difference in AsK $K_{\alpha}$ counts between the substrate and LT layer revealing excess As in the LT layer.

$\mathrm{MeV} \mathrm{H}^{+}$beam was used to excite the layers. The mean penetration depth of a $1.2 \mathrm{MeV} \mathrm{H}+$ beam in GaAs is approximately $8 \mu \mathrm{m}$. The samples were tilted $60^{\circ}$ to the beam to increase excitations in the layer and minimize background counts from the substrate. The ernitted $x$-rays were detected by a $\mathrm{Li}$ drifted silicon, $\mathrm{Si}(\mathrm{Li})$, detector.

Figure 2.1 shows the $x$-ray spectrum of an undoped LEC GaAs substrate and the LT GaAs:Si layer doped at $10^{19} \mathrm{~cm}^{-3}$. The GaK $\alpha$ peaks have been normalized. The two spectra are nearly identical. Careful inspection of the AsK $\alpha$ peak, as seen in the inset of Fig. 2.1 reveals a slight excess in the LT GaAs layer which is statistically significant. The relative composition of the layer, $[\mathrm{As}] /[\mathrm{Ga}]$ can be 
quantitatively determined from the ratio of GaKa and AsK $\alpha$ x-ray counts for the layer and normalized by that taken for the substrate: $[\mathrm{As}] /[\mathrm{Ga}]=\left(\left[\mathrm{AsK}_{\alpha}\right] /\left[\mathrm{GaK}_{\alpha}\right]\right)_{\text {layer }}\left(\left[\mathrm{GaK}_{\alpha}\right] /\left[\mathrm{AsK}_{\alpha}\right]\right)_{\text {substrate. }}$. The detection limit of the PIXE technique is $0.2 \%$ (Yu and Liliental-Weber 1991) as determined by Poisson statistics of $x$-ray counts under the peaks. For the samples grown at $300^{\circ} \mathrm{C}$, the relative composition was determined to be $[\mathrm{As}] /[\mathrm{Ga}]=1.007 \pm .004$. This corresponds to an excess-As stoichiometry ([As]-[Ga])/([As]+[Ga]) $=0.35 \pm 0.2 \%$

\section{$X$-ray Diffraction}

Double crystal $x$-ray diffraction studies utilizing the (400) diffraction peak were made on the LTMBE GaAs layer. Only a single $x$-ray peak, the same as for the substrate, could be observed. Therefore, no expansion of the lattice has occurred due to the $300^{\circ} \mathrm{C}$ temperature growth.

IEM

TEM was performed with the assistance of In Chin $\mathrm{Wu}$ and $\mathrm{Dr}$. Alain Claverie at the National Center for Electron Microscopy. Micrographs of the as-grown layers could not detect the presence of As precipitates in the as-grown material. Precipitates with a diameter greater than $5 \mathrm{~nm}$ would have been detected if present.

The main conclusion to be drawn from the results of structural measurements is that growih at $300^{\circ} \mathrm{C}$ incorporates far less excess As than growth at $200^{\circ} \mathrm{C}$. Hence the material is more structurally perfect than material grown at lower temperatures. Extended defects in the $200^{\circ} \mathrm{C}$ material associated with excess As, such as pyramidal defects, polycrystallinity above a critical thickness, As precipitates and an expanded lattice, are not observed for $300^{\circ} \mathrm{C}$ 
grown material. With very few detectable extended defects the electrical properties should be dominated by the presence of a high concentration of poin $\ddagger$ defects.

\subsubsection{Optical properties}

IR absorption of EL2O-like defects

It is well known that neutral EL20, which is the AsGa-related defect in bulk grown LEC GaAs, exhibits a characteristic intracenter IR absorption.t This characteristic absorption was used to investigate the presence of ASGa-related defects in LTMBE layers. The IR absorption was measured using a CARY model 2300 grating spectrophotometer. An important feature of the CARY is that the light path strikes the diffraction grating before the sample so the sample is illuminated by monochromatic light and not white light. This is critical for the measurement of EL2-like defects which could be unintentionally photoquenched by white light during the measurement at low temperatures. An evacuated cryostat with a liquid $\mathrm{He}$ cold finger was used for the low temperature $(\sim 10 \mathrm{~K})$ measurements.

To prepare the samples for IR absorption measurements, the backside of the substrate had to be polished. Samples were mounted with wax on a polishing jig with a piece of lens paper in between to protect the LTMBE layer. The samples were polished for 15 minutes on a felt pad with a solution of $1: 1: 1$, Syton: $\mathrm{H}_{2} \mathrm{O}_{2}: \mathrm{H}_{2} \mathrm{O}$.

tSee appendix A for a review of the properties of EL2 in GaAs. 


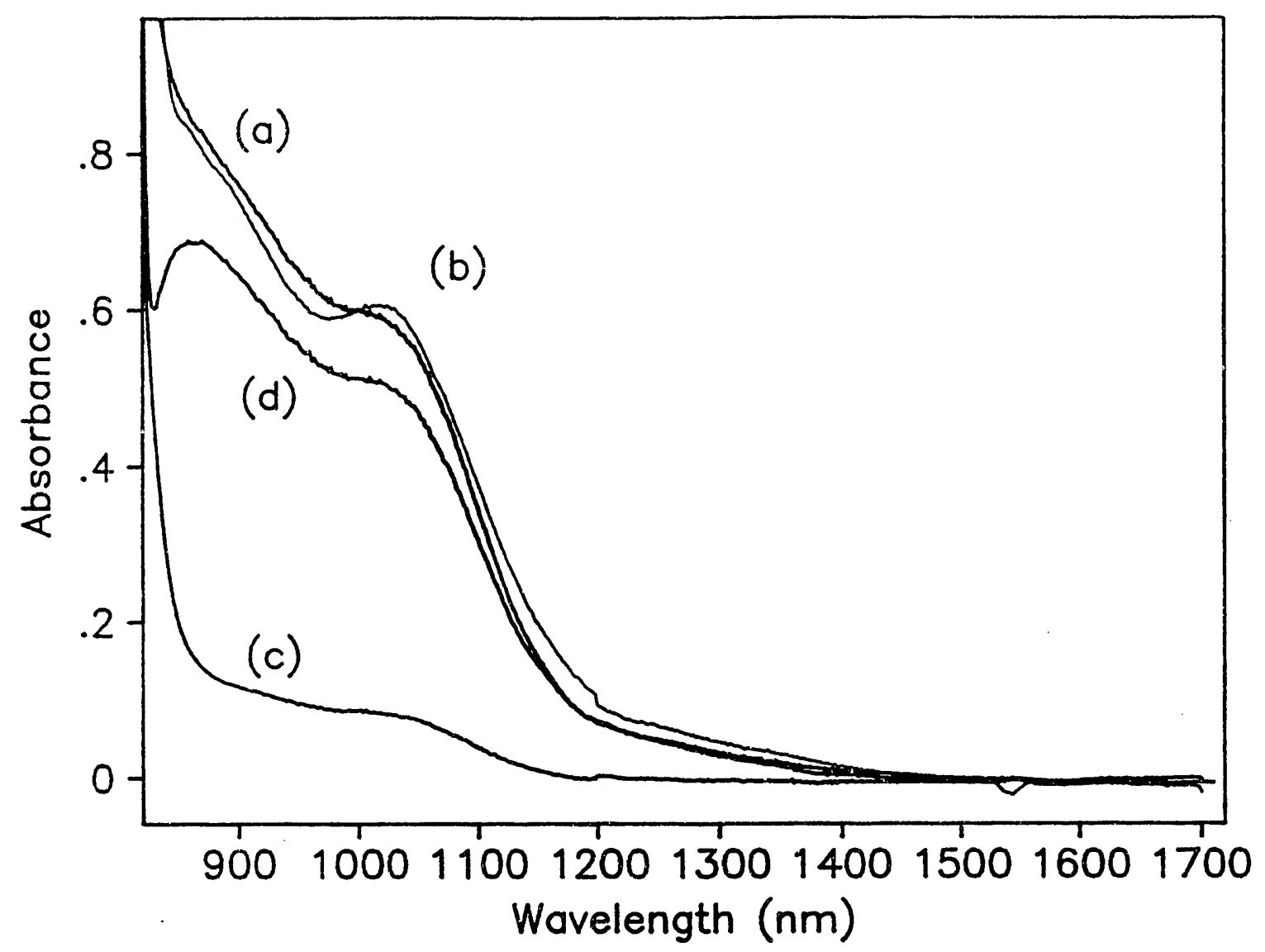

FIG. 2.2 IR absorption spectra of an undoped LTMBE GaAs layer corrected for substrate absorption and LEC GaAs for comparison. (a) $3 \mu \mathrm{m}$ undoped LTMBE GaAs layer, (b) SI L CC GaAs, (c) undoped layer after $30 \mathrm{~min}$. white light illumination, (d) PQ absorption of undoped layer given by the ratio of spectra taken before and after illumination.

Figure 2.2 shows the IR absorption spectra recorded at $10 \mathrm{~K}$ of the undoped LTMBE layer (a) and an SI-LEC GaAs wafer (b) for comparison. The vertical axis is the absorbance, $A=-\log _{10}(T)$, where $T$ is the transmission. The horizontal axis is the photon wavelength, $\lambda(\mathrm{nm})$. Background absorption due to the substrate was measured separately and has been subtracted. The LTMBE spectrum (a) shows the characteristic broad absorption around $1000 \mathrm{~nm}$ similar to EL2 in spectrum (b). Spectrum (b) has been normalized so 
that the two spectra nearly overlay each other displaying the similarity of the two spectra. Although the two spectra are virtually identical, a difference which should be pointed out is the dip in the EL2 absorption just below the broad absorption peak. The SI-LEC wafer shows a well formed EL2 intracenter peak at $1000 \mathrm{~nm}$ and then a dip at $980 \mathrm{~nm}$. The LTMBE layer in contrast, shows no dip. The slope of the absorption never changes sign. Instead it approaches zero, becoming almost flat, and then increases again at the bandedge. The lack of a dip indicates either another defect with an absorption band in the same region could be washing out the EL2like absorption peak or that the peak itself is broadened. This issue will be considered further in the following section on the photoquenching properties.

Another difference between the spectra is the lack of a zero phonon line near $1200 \mathrm{~nm}$ for the LTMBE layer. Figure 2.3 shows the spectra for the LTMBE layer (a) and SI-LEC wafer (b) taken at the best resolution and sensitivity settings of the CARY. The ZPL cannot be detected in the LTMBE layer, but it is clearly visible for the SILEC sample. Because of the lack of ZPL a direct assignment of the defect to EL2 is not made. Rather, it is referred to as EL2-like or AsGa-related.

Martin has shown that the neutral EL2 ${ }^{\circ}$ concentration can be calibrated against the absorption coefficient at $\lambda=1.0 \mu \mathrm{m}$ (Martin 1981, also see appendix A). From his data the optical cross section of EL2O is determined to be $1.3 \times 10^{-16} \mathrm{~cm}^{2}$. Since the AsGa-related, EL2-like defects in LTMBE GaAs are very similar to EL2 a similar estimation of the neutral defect concentration is made. 


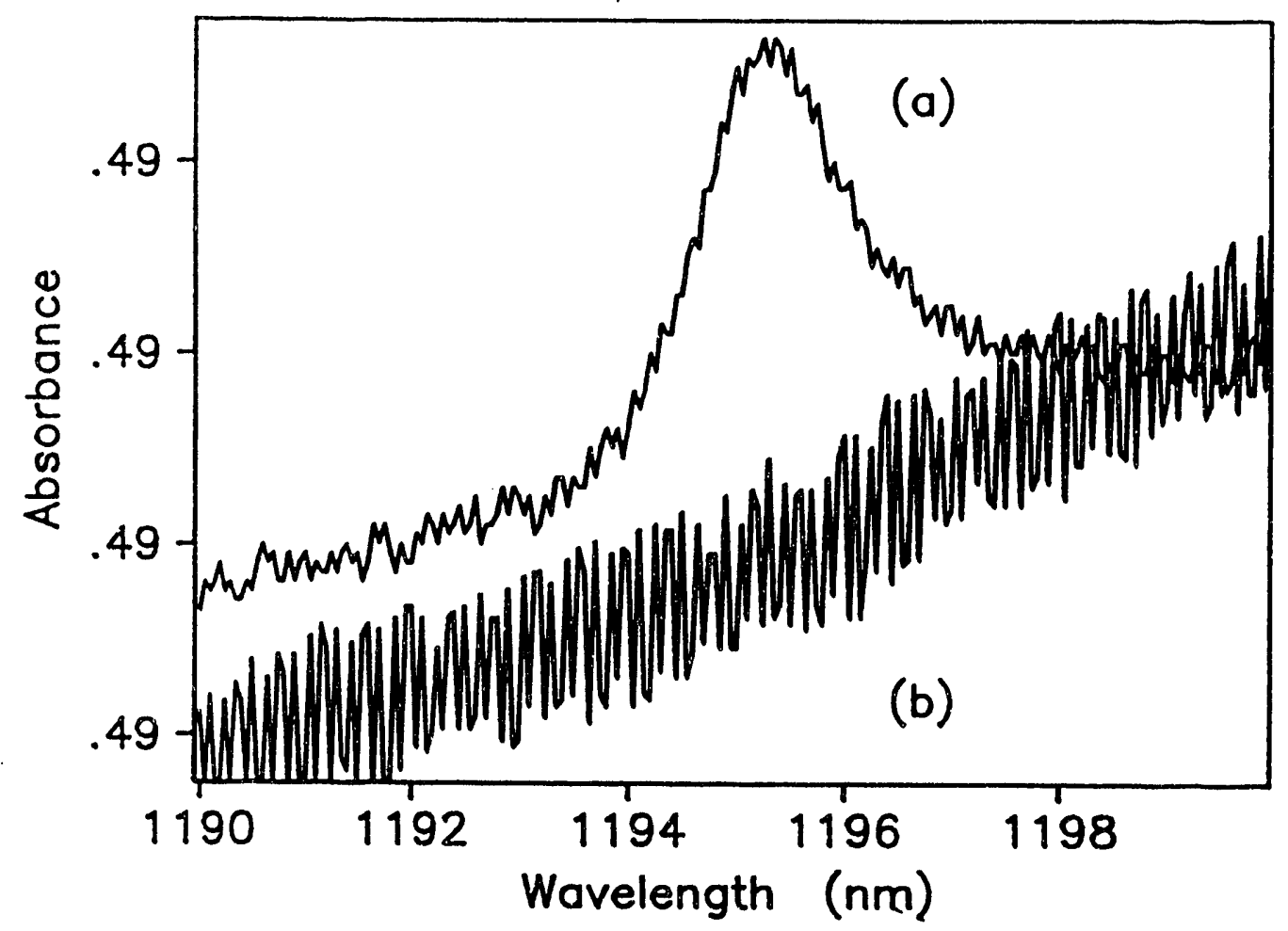

FIG. 2.3 IR absorption spectrum of the Zero Phonon Line (ZPL) of EL2 in (a) SI-LEC GaAs. No ZPL is visible in spectrum (b) of the undoped LTMBE GaAs layer.

However, because the EL2-like defects do not show the exact same spectral features, specifically the lack of a ZPL, a rounded off value of $1 \times 10^{-16} \mathrm{~cm}^{2}$ for the optical cross section will be used to estimate the concentration of EL2-like defects, until an exact calibration has been made.

The absorption at $\lambda=1.0 \mu \mathrm{m}$ is measured relative to absorption at $\lambda=1.7 \mu \mathrm{m}$ which is taken as the background zero reference. The concentration of EL2 ${ }^{\circ}$-like defects in the undoped LTMBE layer determined from the absorption is $5 \times 10^{19} \mathrm{~cm}^{-3}$. This is a typical 
concentration when compared to values of $10^{20} \mathrm{~cm}^{-3}$ (Kaminska 1991) and $4 \times 10^{19} \mathrm{~cm}^{-3}$ (Manasreh, et al. 1990) found by other researchers.

Photoquenching and thermal recovery

The photoquenching studies were performed with white light illumination from a $250 \mathrm{~W}$ projection lamp. Samples were illuminated for 30 minutes. Figure 2.2 (c) and (d) show the results of photoquenching experiments on the undoped layer. Spectrum (c) is the IR absorption after illumination. Most of the absorption at $1 \mu \mathrm{m}$ is quenched by the illumination.

The fraction of $P Q$ defects in the undoped layer is $85 \%$. This is a considerably higher fraction of metastable defects as compared to previously reported results (Manasreh, et al. 1990) which found only $10 \% \mathrm{PQ}$ defects. A higher growth temperature was used for our layers, $300^{\circ} \mathrm{C}$ instead of $200^{\circ} \mathrm{C}$. The strain associated with lower temperature growth could be responsible for the decreased metastability of the AsGa-related defects. Hoinkis and Weber et al. (1989) have proposed that strain in bulk GaAs materials reduces the metastability of EL2. X-ray diffraction studies by Kaminska et al. (1989) of LTMBE layers grown at $200^{\circ} \mathrm{C}$ show these layers to be strained. However, no detectable lattice expansion could be found in their layers grown at $270^{\circ} \mathrm{C}$ or in our layers grown at $300^{\circ} \mathrm{C}$. This is consistent with the observation of a lower concentration of extended and point defects in our layers. Therefore, the reduced strain in layers grown at higher temperatures could lead to a greater $P Q$ fraction of AsGa-related defects. 
Spectrum (d) is the difference between spectra taken before and after illumination showing the shape of the photoquenchable (PQ) absorption. The shape of the $P Q$ absorption (d) is the same as shape of the non-photoquenchable absorption (NPQ) after illumination (c). This indicates that the defects which give rise to the $P Q$ and NPQ $1 \mu \mathrm{m}$ absorption band are closely related. Because the shape of the PQ and NPQ are the same, it also implies that the lack of a dip at $980 \mathrm{~nm}$ is due to broadening of the EL2-like peak and not due to absorption from another defect washing out the EL2-like absorption. We see that whatever mechanism prevents the metastability, does not affect the intracenter transition to an excited state. Rather, it must prevent the relaxation of the excited state into the metastable state. Varying strain fields in the vicinity of defects could be a possible mechanism broadening the peak. It is known that the position of the broad peak for EL2 is a function of pressure (Baj and Dreszer 1989). They found the pressure derivative of the EL2 absorption peak to be $-26.4 \mathrm{meV} \cdot \mathrm{GPa}-1$.

The metastable state of EL2 can be thermally recovered by warming the sample above $130 \mathrm{~K}$. Figure 2.4 shows the IR absorption at $1 \mu \mathrm{m}$ versus the sample temperature. The increase in absorption at $130 \mathrm{~K}$ indicates that EL2-like defects in LTMBE GaAs recover at the same temperature as EL2. A general rule of thumb to estimate the energy barrier for a thermally activated process involves dividing the recovery temperature, $T_{0}(K)$ by 0.4 to get the activation energy $E_{a}$ in meV, 


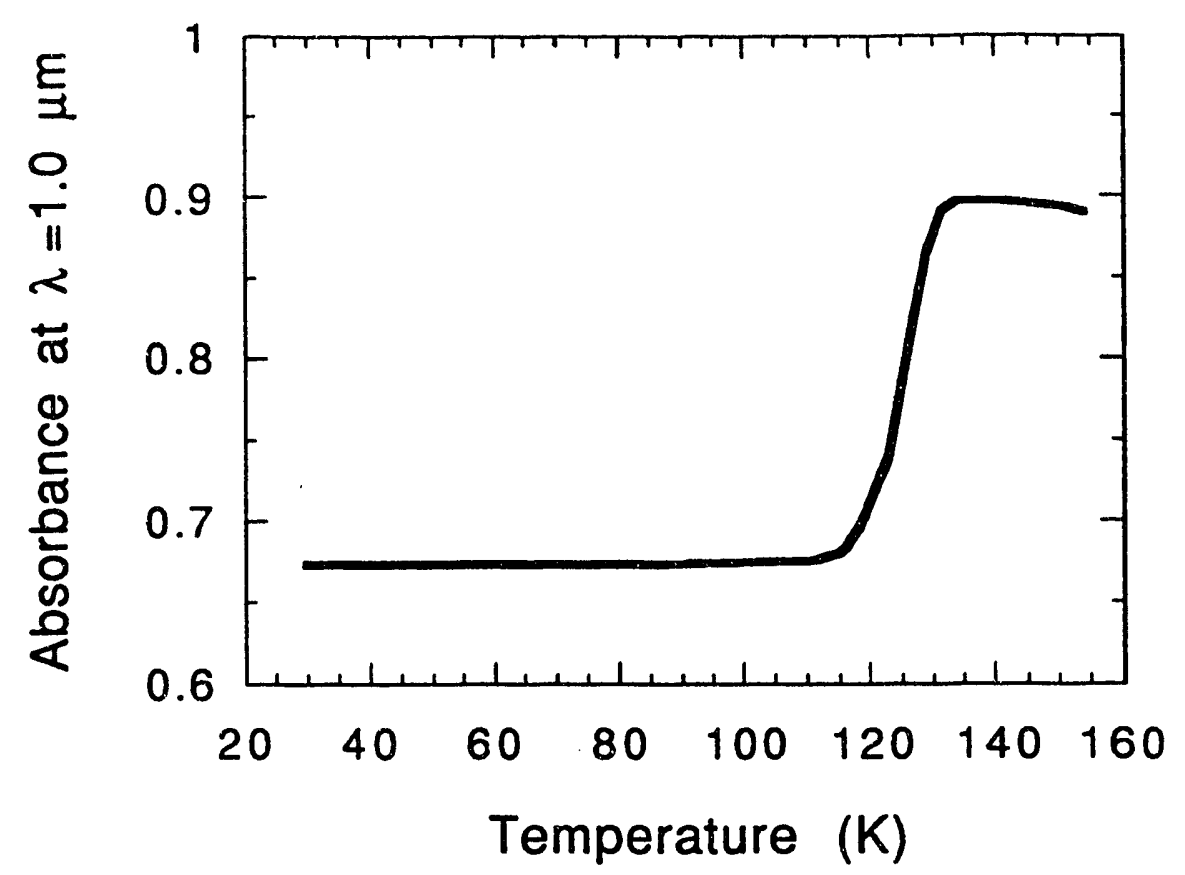

FIG. 2.4 IR absorption at $\lambda=1 \mu \mathrm{m}$ versus temperature illustrating the thermal recovery of photoquenched EL2-like defects. Recovery is complete after reaching a temperature of $140 \mathrm{~K}$.

$$
E_{a}=\frac{T_{0}}{0.4}
$$

A value of $0.33 \mathrm{eV}$ is obtained for the energy barrier for the recovery of EL2-like defects from the metastable state.

Positron Annihilation

Another point defect in GaAs which can accommodate excess As, besides $A s G a$, is $V_{G a}$. Unlike EL2, $V_{G a}$ does not have an identifiable electrical or optical characteristic which could be utilized to determine its concentration. It is well known, however, that the rate of annihilation of positrons in GaAs is sensitive to the concentration of negatively charged $\mathrm{V}_{\mathrm{Ga}}$. This fact has been used to estimate $\left[V_{G a}\right]$ from the positron lifetime in bulk crystals (Dlubek 
and Kruse 1987, See e.g. Fig. 14). Due to a large penetration depth of the high energy positrons from a radioactive source, this type of study is not appropriate for thin layers. In this case one has to resort to the use of moderated, lower energy, "slow" positrons. The positrons from the radioactive source are moderated by scattering in a thin tungsten foil, and then extracted by an applied potential. Once the positron has been moderated, however, it is no longer possible to determine its lifetime. Instead, one must analyze the spectrum of $\gamma$-rays emitted by the annihilation of the slow positron. The concentration of $\left[\mathrm{VGa}_{\mathrm{Ga}}\right.$ can be related to the $S$ parameter of the $\gamma$ ray spectrum. The spectrum of $\gamma$-rays shows two characteristic features, a sharp peak due to annihilations with conduction electrons and a broad background due to annihilations with core electrons. The broad background is a result of Doppler broadening by the higher momentum core electrons. In a crystal containing neutral or negatively charged vacancies which can trap positrons, the annihilation will occur selectively with conduction electrons since there are no core electrons at a vacancy. Thus there will be more $\gamma$-ray counts in the central portion of the spectrum. The $S$ parameter, which is convenient to think of as the "Sharpness" parameter, is the ratio of the central $511 \mathrm{keV} \gamma$ ray counts (accumulated between 510.5 to $511.5 \mathrm{keV}$ ) to the total number of counts. It will be correspondingly larger in the material containing $V_{G a}$. Thus, the $S$ parameter, an inverse measure of the Doppler broadening, is a measure of the $V_{G a}$ concentration. More detailed discussions of slow positron annihilation measurements and Doppler 
broadened $\gamma$-ray spectroscopy can be found in the literature (Lee, et al. 1990).

Slow positron annihilation experiments were performed in collaboration with Prof. Shoichiro Tanigawa's group at the Institute of Materials Science, University of Tsukuba, Japan. The results of the slow positron annihilation experiments are shown in Fig. 2.5. The S-parameter versus positron implantation energy is shown for the LEC substrate and undoped as-grown LTMBE GaAs layer. The implantation energy controls the penetration depth of the positron. The dependence of the mean penetration depth $\bar{x}(\mathrm{~nm})$ on the acceleration energy $E(\mathrm{keV})$ is given by the empirical expression

$$
\bar{x}=\left(\frac{40}{\rho}\right) E^{1.6}
$$

where $\rho$ is the material density $\left(5.31 \mathrm{~g} \cdot \mathrm{cm}^{-3}\right.$ for GaAs). At the highest implantation energy, $E=10 \mathrm{keV}$, the penetration depth is $\sim 0.4 \mu \mathrm{m}$, so that the positrons annihilate well within the $3 \mu \mathrm{m}$ layer.

For positrons implanted to a shallow depth, at energies below $5 \mathrm{keV}$, the S-parameter increases as the energy tends to zero (see Fig. 2.5). This is a surface effect and is seen in all samples. Positrons not trapped at defects can diffuse through the crystal before annihilating. A positron near the surface can diffuse and be trapped at the surface where it is more likely to annihilate with an electron other than a core electron. The S-parameter is therefore inflated in the near surface region. To illustrate this effect, 


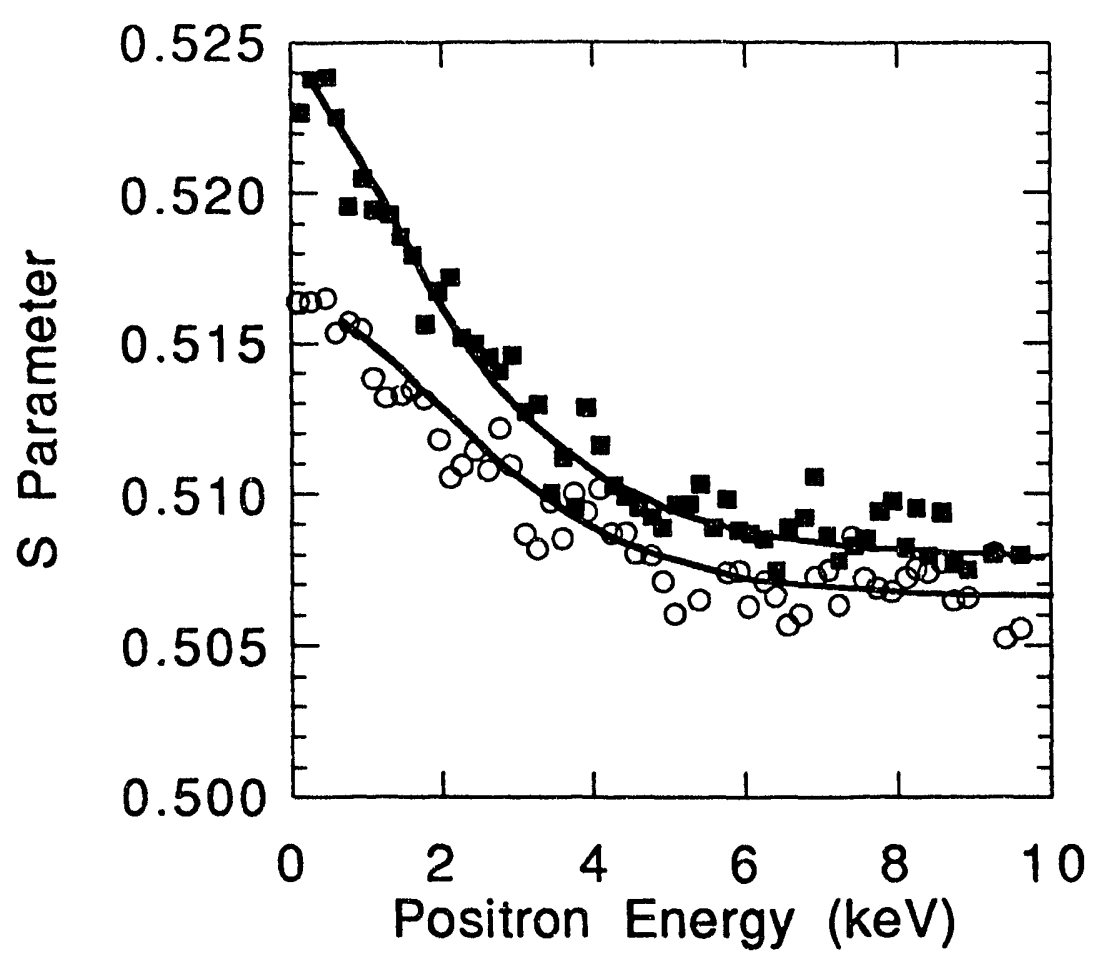

FIG. 2.5 Results of slow positron annihilation investigations of undoped LTMBE GaAs layers. The S-parameter is plotted vs. the positron implantation energy. (0) LEC substrate. (E) undoped LTMBE GaAs layer. The solid lines are the best fit to the data using eq. 2.3.

consider the following simple calculation. The diffusion length of free positrons in GaAs can be roughly estimated by $\sqrt{D \tau}$ where $D$ is the diffusivity and $\tau=10-10 \mathrm{~s}$ is the free positron lifetime. The Einstein relation, $D=\mu k T / e$, is used to get $D$ from the mobility, $\mu$. The mobility for positrons will be roughly the same as for electrons since they see the same periodic atomic potential. Using a value of $\mu=10^{3} \mathrm{~cm}^{2} \mathrm{~V}^{-1} \mathrm{~s}^{-1}$ gives a diffusion length of $0.4 \mu \mathrm{m}$ at $300^{\circ} \mathrm{C}$. This value will depend of course on the presence of electric fields near the surface, but it is of the right magnitude. A more detailed description of the diffusion of positrons to the surface, including references can be found in the review by Dlubek et al. (1987). 
The LTMBE layer exhibits a larger S-parameter than the LEC substrate which indicates that a larger concentration of $\mathrm{V}_{\mathrm{Ga}}$ are present in these layers. To get an estimate of the extra concentration of $V_{G a}$ in the LTMBE layer the energy dependence of $S(E)$ profile will be analyzed in a more detailed fashion. As pointed out previously the $S(E)$ response is the sum of the annihilation at the surface, $S_{s}$, and in the bulk $S_{b}$, expressed as

$$
S(E)=S_{s} F_{s}(E)+S_{b}\left[1-F_{s}(E)\right]
$$

where $F_{S}(E)$ is the fraction of positrons annihilating at the surface. $F_{S}(E)$ is given by the expression

$$
F_{S}(E)=\int_{0}^{\infty} P(x, E) \exp \left[\frac{-x}{L}\right] d x
$$

where $P(x, E)$ is the implantation profile of positrons (Schulz and Lynn 1988) and $L$ is the positron diffusion length. The concentration of $V_{G a}$ is obtained from the value of $L$. The relation $L=\sqrt{D_{\downarrow} / \kappa_{\text {eff }}}$ is the expression for the diffusion length in terms of the positron diffusivity, $D_{+}$, and the time the positron can diffuse $\tau=1 / \kappa_{\text {eff }}$. The net escape rate, $\kappa_{e f f}$, is the loss of positrons from the freely diffusing state. Note, $\kappa_{\text {eff }}$ is not the inverse of positron lifetime. It is due to both the annihilation of positrons and the trapping of positrons by defects and can be expressed as

$$
\kappa_{\text {eff }}=\lambda_{f}+\mu_{d} C_{d}
$$


TABLE 2.1 Summary of the fitted parameters for slow positron annihilation indicating the bulk $S$ parameter, $S_{b}$, the surface $S$ parameter, $S_{S}$, the diffusion length, $L$, and the excess concentration of $V_{G a}$ derived from $L$.

\begin{tabular}{lcccc}
\hline \multicolumn{1}{c}{ Sample } & $S_{b}$ & $S_{s}$ & $L(\AA)$ & $\begin{array}{c}\Delta\left[V_{G a l}\right. \\
\times 10^{19}\left(\mathrm{~cm}^{-3}\right)\end{array}$ \\
\hline $\begin{array}{l}\text { LEC GaAs } \\
\text { LTMBE GaAs: }\end{array}$ & 0.50618 & 0.51654 & 361 & $\ldots$ \\
Undoped & 0.50748 & 0.52419 & 317 & 1.18 \\
\hline
\end{tabular}

where $\lambda_{f}$ is the free positron annihilation rate, $\mu_{d}$ is the positron trapping probability by the defect and $C_{d}$ is the defect concentration. Even though the positron lifetime can become longer once it is trapped, this is not important. What is meaningful in this analysis is the trapping time so that the positron can no longer diffuse.

Figure 2.5 show the $S$ parameter vs. positron energy for the SILEC substrate and undoped LTMBE GaAs layer. The solid curves represent the best fit using eq. 2.3. $S_{b}$, the bulk annihilation $S$ parameter is determined by averaging the $S$ values in the energy range from 15 to $30 \mathrm{keV}$. The parameters obtained by this fit are summarized in Table 2.1 for the undoped LTMBE layer.

The $V_{G a}$ concentration is estimated from the fitted values of $L$ by using eq. 2.5. The main error of approximately one order of magnitude is due to the fact that the exact value of $\mu_{d}$ is unknown. Theoretical calculations (Puska, et al. 1990) suggest values for the trapping by neutral and negatively charged vacancies of $4.4 \times 10^{14}$ and $2.5 \times 10^{15} \mathrm{~s}^{-1}$ respectively. For this analysis the value of $2.5 \times$ $1015 \mathrm{~s}^{-1}$ was used to estimate the concentration of negatively charge $V_{G a}$-related defects. 
One point to consider is that the positron diffusion length measured for our SI-LEC GaAs substrate $(360 \AA)$ is shorter than reported in the literature for defect free bulk GaAs (1000-3000 $)$ (Lee, et al. 1992). This is low value is disconcerting since it implies the presence of a high $\mathrm{V}_{\mathrm{Ga}}$ concentration in the substrate already. However, any mechanism which prevents positrons from annihilating at the surface will reduce the measured diffusion length. Most likely the shorter diffusion length is due to oxidation of the surface. The samples had been grown and exposed to air for one year before the positron annihilation experiments. It is known that surface oxides from prolonged exposure of GaAs to air (10 days or more) can influence the $S(E)$ response, (Lee, et al. 1990) reducing the measured positron diffusion length. Lee attributes the effect to the formation of defects at the surface which can trap positrons. A shift in the Fermi level pinning position at the surface will alter the surface electric fields and also influence the diffusion of the charged positron. If the surface pinning level moves higher in the bandgap, positrons will be repelled from the surface decreasing the apparent diffusion length. Because of the difference between the measured positron diffusion length and values reported in the literature, we can only make comparisons amongst our samples. Therefore, the difference in vacancy concentration, $\Delta\left[V_{G a}\right]$, between the substrate and the LTMBE layers is calculated. The excess gallium vacancy concentration, $\Delta\left[\mathrm{V}_{\mathrm{Ga}}\right]$, obtained for the undoped LTMBE layer is on the order of $10^{19} \mathrm{~cm}^{-3}$.

Although this is a rather involved means to get an order of magnitude estimate of the $V_{G a}$ concentration, it is so far the only 
way to obtain any indication of the existence of vacancies in these LTMBE layers. The fact that the LTMBE layer exhibits a larger Sparameter than the LEC substrate over the entire energy range and that the positron diffusion length is shorter, indicates that a larger concentration of $\mathrm{V}_{\mathrm{Ga}}$ are present in the LTMBE layers. Even though it is difficult to be quantitative about the concentration of $\mathrm{V}_{\mathrm{Ga}}$, positron annihilation experiments provide important qualitative information about the presence of excess $V_{G a}$ in LTMBE GaAs.

\subsubsection{Electrical properties}

The electrical properties of the LTMBE layers were measured by variable temperature Hall effect with the sample in the van-derPauw configuration. As grown, the layer is more conductive than the SI substrate so parallel conductivity in the substrate is not a problem. Figure 2.6 shows results of variable temperature Hall effect and resistivity measurements of the undoped LTMBE GaAs layer. The temperature dependence of the resistivity shows two regions where it depends linearly on the inverse temperature. The temperature dependence of the resistivity $\rho$ can be expressed as (Shklovskii and Efros 1984)

$$
\frac{1}{\rho}=\frac{1}{\rho_{1}} \exp \left(-\frac{\varepsilon_{1}}{\mathrm{kT}}\right)+\frac{1}{\rho_{3}} \exp \left(-\frac{\varepsilon_{3}}{\mathrm{kT}}\right)
$$

where $k$ is Boltzmann's constant and $T$ is the temperature. $\rho_{1}$ and $\varepsilon_{1}$ are the extrapolated infinite temperature intercept and the activation energy respectively of the resistivity above room 


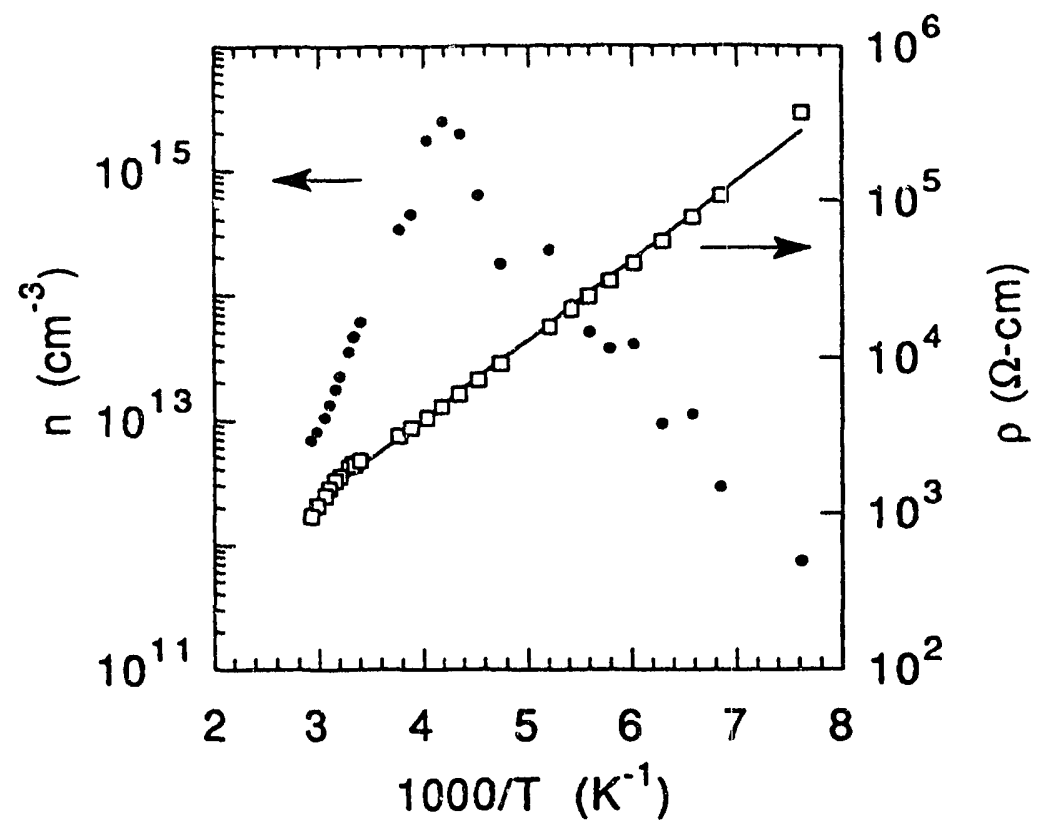

FIG. 2.6 Plot of varia.ole temperature Hall effect and resistivity for an undoped LTMBE GaAs layer.

temperature $\left(1000 / T<3.3 \mathrm{~K}^{-1}\right)$ and correspondingly $\rho_{3}$ and $\varepsilon_{3}$ describe the resistivity below room temperature.

Above room temperature the resistivity decreases rapidly as the temperature is increased. The slope corresponds to an activation energy $\varepsilon_{1}=0.75 \mathrm{eV}$. This is just normal free carrier conduction due to the thermal excitation of electrons into the conduction band from the Fermi level positioned at midgap. One must consider that the LTMBE layer and the SI substrate are conducting in parallel. The value of the sheet resistance calculated for the $3 \mu \mathrm{m}$ layer above room temperature is the same as the sheet resistance of the $0.5 \mathrm{~mm} \mathrm{SI} \mathrm{subitrate.} \mathrm{This} \mathrm{leads} \mathrm{us} \mathrm{to} \mathrm{believe} \mathrm{that}$ above room temperature we are actually measuring the electrical properties of the substrate and not the layer. 
Below room temperature the resistivity of the layer is much less than the SI substrate so that the electrical conduction occurs only in the layer. In this temperature range, the resistivity is thermally activated with an activation energy $\varepsilon_{3}$. As the temperature is decreased further below room temperature, the mobility drops below $1 \mathrm{~cm}^{2} \mathrm{~V}^{-1} \mathrm{~s}^{-1}$ and the Hall concentration measurement becomes unreliable (see Fig. 2 6). This type of behavior is indicative of electrons hopping from one localized state to another (Shklovskii and Efros 1984). The exponential dependence of the resistivity on inverse temperature is characteristic of nearest neighbor hopping conduction as opposed to variable range or Mott hopping conduction. The activation energy for this process is due to the dispersion of the hopping center energy levels by the random Coulomb interactions of hopping centers. The magnitude of the activation energy $\varepsilon_{3}$ reflects the energy difference between the Fermi level and the unperturbed energy level of the defect as shown in Fig. 2.7. In other words, for a lightly compensated hopping level, $\varepsilon_{3}$ is the amount of spreading or dispersion of the hopping level. For the LTMBE layers the hopping centers would be the partially occupied AsGa-related defects. We find the activation energy $\varepsilon_{3}=99 \pm 3 \mathrm{meV}$

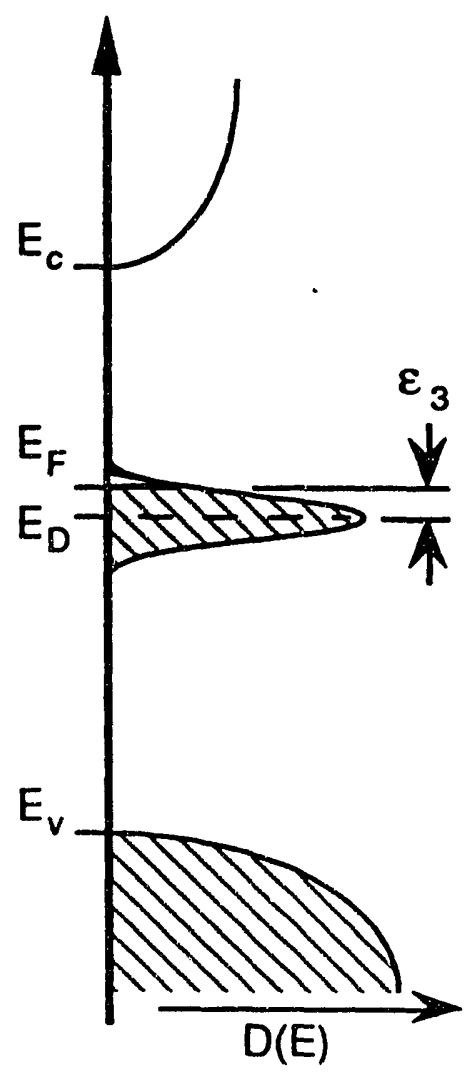

FIG. 2.7 Schematic diagram of the density of states for a defect level in the bandgap indicating the activation energy $\varepsilon_{3}$ for hopping conductivity. 
It has been shown by others (Kaminska and Weber 1990, Look, et al. 1990) that in LTMBE GaAs grown at even lower temperatures the resistivity showed a temperature dependence $\rho=\rho_{0} \exp \left[\left(T / T_{0}\right)^{-}\right.$ 1/4] which is characteristic for variable range hopping conduction. This behavior was observed for temperatures lower than $150 \mathrm{~K}$. In our measurements we do not clearly see any indication that variable range hopping plays any role in our undoped layers.

Hopping conduction via localized defect states is not unique to LTMBE GaAs and has been observed in high energy particle damaged GaAs. Particularly careful studies of the electrical properties of GaAs as function of damage created by various implanted ions were performed by Kato et al. (1974). Extensive studies of neutron irradiation damaged GaAs have been reviewed by Coates and Mitchell (Coates and Mitchell 1975).

\subsubsection{Hydrogen plasma passivation}

Many electronic levels in semiconductors, from shallow donors and acceptors to deep level impurities and defects, can be passivated by hydrogen. Specifically, it has been documented that both shallow donors (Pearton, et al. 1986) and acceptors (Johnson, et al. 1986) in GaAs are passivated by hydrogen. Of great technological importance is the passivation of dangling bonds in amorphous and polycrystalline Si (Paesler, et al. 1989) for inexpensive solar cell applications. It has also been observed by DLTS that the deep defect level EL2 in LEC GaAs can be passivated by a hydrogen plasma (Lagowski, et al. 1982). With this in mind we set out to observe by IR absorption, the hydrogen passivation of EL2-like defects in LTMBE GaAs layers but were unsuccessful. 
Samples were hydrogen plasma passivated in collaboration with Noble Johnson at Xerox PARC. The plasma passivation chamber is designed to isolate the sample from energetic hydrogen ions in order to avoid damage to the sample. The layers were heated to $300^{\circ} \mathrm{C}$ and exposed to the hydrogen plasma for 1 hour. In addition, a control sample was heated to $300^{\circ} \mathrm{C}$ in the chamber, but not exposed to a hydrogen plasma. The temperature is elevated to enhance the diffusion of the hydrogen into the sample. Under these conditions hydrogen penetrates about $1 \mu \mathrm{m}$ in bulk LEC GaAs.

IR absorption spectra of the intracenter absorption at wavelengths around $\lambda=1 \mu \mathrm{m}$ were taken before and after plasma treatment. No difference could be observed in either the control or the plasma treated sample. Thus we see no evidence at this point for the passivation of EL2-like defects. To determine if the hydrogen is actually diffusing into the LTMBE GaAs layers, one sample was passivated with deuterium. Deuterated samples are necessary for SIMS measurement because the hydrogen background of the system is too high. An undoped LTMBE layer was maintained at a temperature of $350^{\circ} \mathrm{C}$ and was exposed to a deuterium plasma for 60 minutes. The SIMS profile of the deuterated sample is shown in Fig. 2.8. The deuterium does not appear to have been able to diffuse into the layer, having penetrated only a few hundred $\AA$. One possible explanation for the observed lack of passivation is that the hydrogen is being effectively trapped by defects and cannot diffuse into the sample. Formation of molecular hydrogen in the near surface region may further slow down hydrogen diffusion. This suggests that it might be possible to passivate the defects if only hydrogen can be 


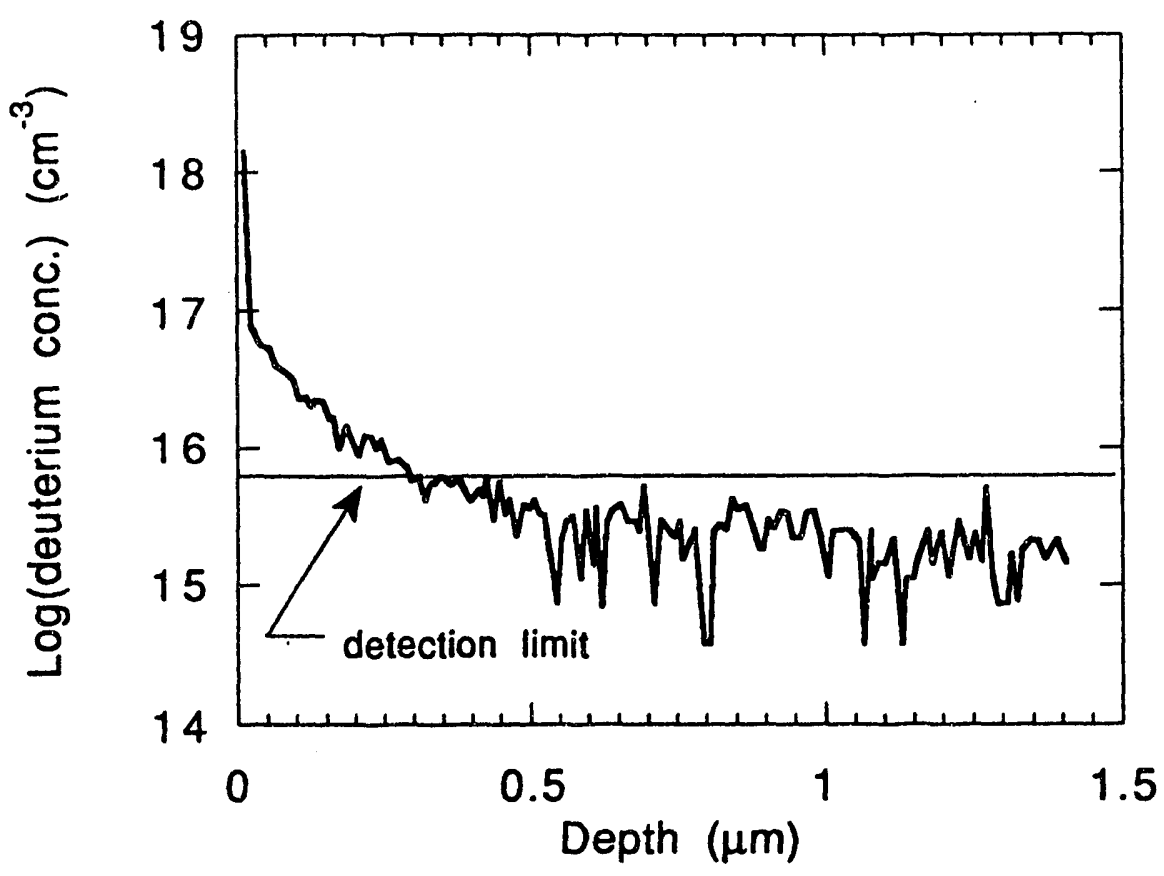

FIG. 2.8 SIMS profile of a deuterium plasma treated undoped LTMBE

GaAs layer. The sputter rate and secondary ion yield have been calibrated to provide a plot of the Logarithm of the deuterium concentration vs. depth .

introduced into the sample. In fact defects near the surface responsible for trapping the hydrogen are being passivated. Similar trapping effects limiting the diffusion of hydrogen have been observed in $\alpha$-Si passivation (Jackson and Tsai 1992).

\subsection{Characterization of doped layers}

\subsubsection{Growth conditions}

$\mathrm{Be}$ and $\mathrm{Si}$ were introduced in the layers during growth in a wide range of concentrations at $10^{16}, 10^{17}, 10^{18}$ and $10^{19} \mathrm{~cm}^{-3}$. To vary the dopant concentration the flux of atoms was adjusted by controlling the MBE evaporation source temperature. There was some concern that the substrate might be heated by IR radiation from the doping source. This does not appear to have been a problem, though. The concentration AsGa-related defects remained essentialiy constant or increased in the highest $\mathrm{Be}$ and $\mathrm{Si}$ doped 
layers, respectively. If the samples had been heated the excess As concentration would have been expected to decrease.

\subsubsection{Secondary Ion Mass Spectrometry (SIMS)}

SIMS measurements were made to determine the total concentration of dopants incorporated in the layer. SIMS measurements can detect an impurity element in a matrix without regard to how it is incorporated as long as the concentration is above the detection limit. All atoms, whether substitutional, interstitial or in precipitates are observed. In brief, a SIMS measurement consists of sputtering away the sample with an ion beam. The ejected sample material which is ionized is then scanned in a mass spectrometer to determine its content. Although simple in principle, SIMS measurements require expertise in order to obtain meaningful results. Choice of the primary ion sputtering species, the secondary ion detection species, acceleration and extraction potentials, careful depth and yield calibration, plus many other parameters are critical to an accurate SIMS measurement.

SIMS measurements were performed by Charles Evans Associates. A $\mathrm{Cs}^{+}$beam was used to sputter the sample. The

TABLE 2.2 A summary of the Be and Si concentrations in intentionally doped LTMBE GaAs as determined by SIMS measurements. Calibration of the concentrations from ion implanted standards are accurate to within a factor of two.

\begin{tabular}{cc}
\hline $\begin{array}{c}\text { Intended Doping } \\
\left(\mathrm{cm}^{-3}\right)\end{array}$ & $\begin{array}{c}\text { Concentration } \\
\text { measured by SIMS } \\
\left(\mathrm{cm}^{-3}\right)\end{array}$ \\
\hline Be $10^{18}$ & $3.0 \times 10^{18}$ \\
Be $10^{19}$ & $3.0 \times 10^{19}$ \\
Si $10^{18}$ & $1.5 \times 10^{18}$ \\
Si $10^{19}$ & $2.0 \times 10^{19}$ \\
\hline
\end{tabular}


detected species were the $\mathrm{Si}+$ ion and the charged $\mathrm{Be}-\mathrm{As}^{+}$pair for the $\mathrm{Be}$ doped layers. The sputter yield of $\mathrm{Be}-\mathrm{As}$ pairs is greater than for isolated $\mathrm{Be}$ ions, thereby providing a more accurate concentration measurement. The count rate was calibrated against a known $\mathrm{Be}$ ion implanted sample and is accurate to within a factor of two. Table 2.2 summarizes the SIMS results, confirming that $\mathrm{Be}$ and $\mathrm{Si}$ is incorporated in the film at the intended concentrations.

\subsubsection{PIXE}

The results of PIXE measurements on the doped layers to determine the non-stoichiometry are summarized in Table 2.3. The ratio $[A s] /[G a]=\left(\left[A K_{\alpha}\right] /\left[G a K_{\alpha}\right]\right)$ layer $\left(\left[G a K_{\alpha}\right] /\left[\right.\right.$ AsK $\left.\left._{\alpha}\right]\right)$ substrate and the corresponding excess As percentage are tabulated. The amount of excess $A s$ is just above the detection limit except for the $10^{19} \mathrm{~cm}^{-3}$ $\mathrm{Si}$ doped layer. In this sample the amount of excess As is significantly greater. Why the excess $A s$ is incorporated into the heavily Si-doped layers is an interesting question which will be considered later in this chapter.

TABLE 2.3 Excess As in undoped, Be doped and Si doped LTMBE GaAs as determined by PIXE measurements.

\begin{tabular}{ccc}
\hline $\begin{array}{c}\text { Sample Doping } \\
\left(\mathrm{cm}^{-3}\right)\end{array}$ & $\begin{array}{c}{[\mathrm{As}] /[\mathrm{Ga}]} \\
( \pm 0.003)\end{array}$ & $\begin{array}{c}\text { Excess As } \\
( \pm 0.2 \%)\end{array}$ \\
\hline Undoped & 1.0069 & 0.34 \\
Be $10^{17}$ & 1.0027 & 0.14 \\
Be $10^{19}$ & 1.0021 & 0.11 \\
Si $10^{17}$ & 1.0024 & 0.12 \\
Si $10^{19}$ & 1.0173 & 0.86 \\
\hline
\end{tabular}




\subsubsection{IR Absorption}

IR absorption measurements were performed on the Be doped samples in order to determine the concentration of of neutral AsGarelated defects. Figure 2.9 shows the IR absorption spectra and photoquenching (PQ) properties of all the Be doped layers. Spectrum (a) is of a SI-LEC wafer showing the absorption of EL2 as a comparison. Again, like the undoped layer, the shape of the absorption in the Be doped material does not show as pronounced a dip before the broad EL2-like absorption around $1000 \mathrm{~nm}$. Table 2.4 lists the values for the concentration of neutral AsGa-related defects. The highest concentration is $3 \times 10^{19} \mathrm{~cm}^{-3}$ for the 1017 $\mathrm{cm}^{-3}$ doped sample. Increased $\mathrm{Be}$ doping reduced the concentration

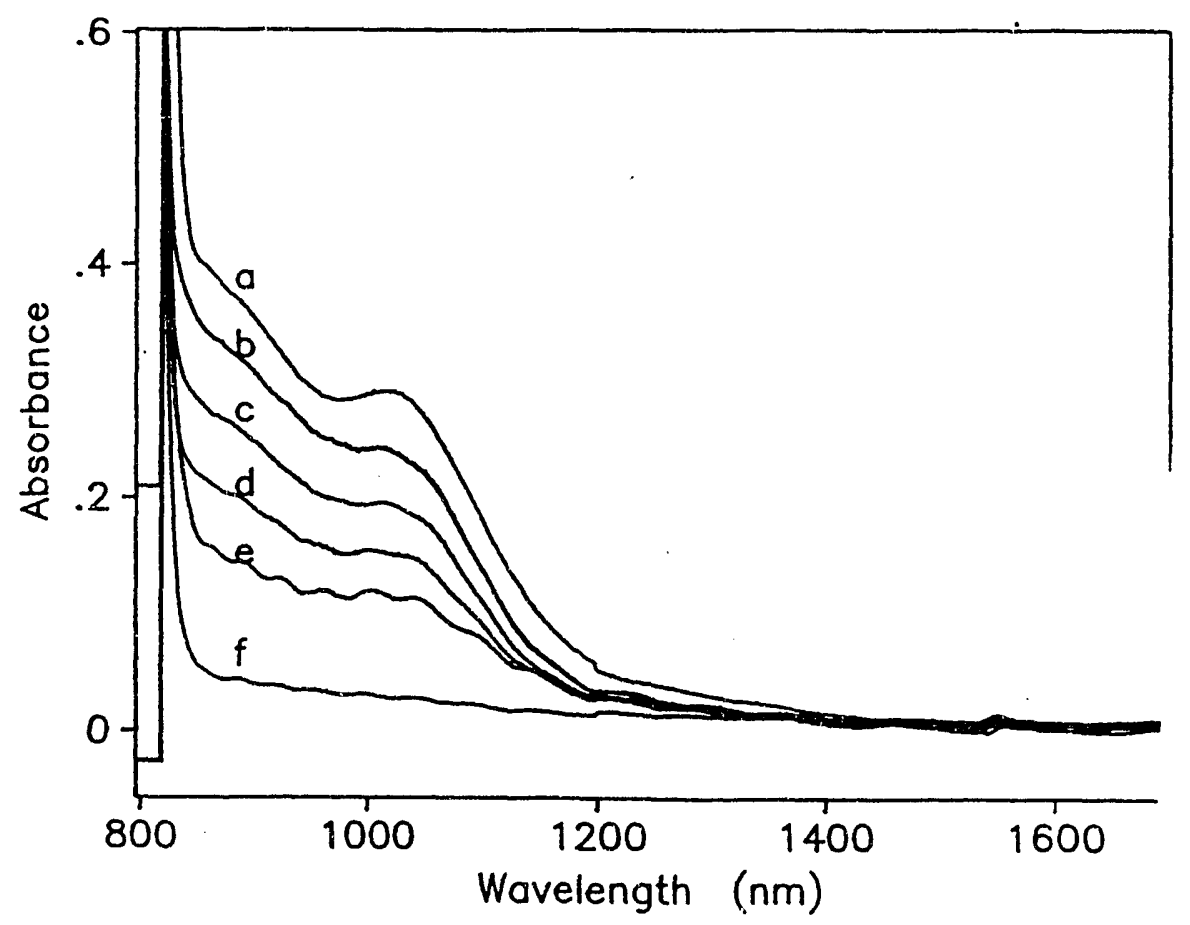

FIG. 2.9 IR absorption spectra of LTMBE GaAs:Be layers corrected for substrate absorption. (a) SI-LEC substrate, (b) $10^{17} \mathrm{~cm}^{-3}$ doped layer, (c) $10^{16}$ $\mathrm{cm}^{-3}$, (d) $10^{18} \mathrm{~cm}^{-3}$, (e) $10^{19} \mathrm{~cm}^{-3}$, (f) $10^{18} \mathrm{~cm}^{-3}$ doped layer after 30 min. white light illumination. 
of neutral AsGa-related defects to $1.5 \times 10^{19} \mathrm{~cm}^{-3}$. If we consider the donor like AsGa-related defects to be compensated by $\mathrm{Be}$, then the total concentration $\left[A \mathrm{AGa}_{\mathrm{a}}\right.$ ] for the $[\mathrm{Be}]=1019 \mathrm{~cm}^{-3}$ doped sample would be $2.4 \times 10^{19} \mathrm{~cm}^{-3}$. This indicates that the total concentration of AsGa remains roughly constant and does not depend on the Be concentration.

Spectrum (f) shows the photoquenching properties of the 1018 $\mathrm{cm}^{-3}$ doped sample indicating that nearly all of the absorption can be photoquenched. The $P Q$ fraction of defects ranged from $56 \%$ to $86 \%$. Comparing our IR absorption results of LTMBE GaAs:Be to previously reported results (Manasreh, et al. 1990) on undoped layers, we find a very significant difference in the $P Q$ fraction of AsGa-related defects. Similar to our undoped material, the Be-doped layers contain a much higher fraction, about $85 \%$, of photoquenchable defects. This can be contrasted against a $10 \%$ fraction of $P Q$ defects in other undoped LTMBE material (Manasreh, et al. 1990). The PQ fraction in our measurements does not depend on the $\mathrm{Be}$ concentration except for the $[\mathrm{Be}]=10^{19} \mathrm{~cm}^{-3}$ doped sample where it decreases to $56 \%$. However, considering that many of the AsGarelated defects are compensated by the $10^{19} \mathrm{~cm}^{-3} \mathrm{Be}$ acceptors, $t$ the actual $P Q$ fraction is more likely to be greater than $75 \%$. The $P Q$ fraction of defects does not depend on the concentration of $\mathrm{Be}$ and is similar to the $P Q$ fraction of the undoped layer. These two facts lead us to believe that the higher growth temperature $\left(300^{\circ} \mathrm{C}\right.$

T EPR measurements discussed later in this section show that the AsGa-related defect is compensated primarily by the Be shallow acceptors. 
instead of $200^{\circ} \mathrm{C}$ ) and not the presence of $\mathrm{Be}$, is responsible for the

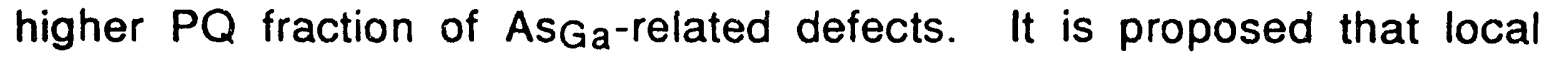
strains from nearby defects in the LTMBE layers reduce the metastability of EL2-like defects by disrupting the the large lattice relaxation.

Figure 2.10 shows IR absorption spectra for the Si doped samples and for a SI-LEC GaAs wafer for comparison. All the LTMBE samples show a strong absorption before the bandgap. The shape of the absorption, which is similar to the spectra of the Be doped samples, differs slightly from the shape of the EL2 shown in spectrum 2.18a. The dip after the broad absorption at $1.0 \mu \mathrm{m}$ is missing. Instead, the slope of the absorption decreases slightly before increasing at the bandgap. The lack of a dip in the Si doped samples is even more pronounced than for the Be doped samples.

TABLE 2.4 Concentration of EL20-like defect in LTMBE GaAs:Be.based on IR absorption measurements. $P Q$ fraction is determined after $30 \mathrm{~min}$. white light illumination.

\begin{tabular}{ccc}
\hline $\begin{array}{c}\text { Be } \\
\text { concentration } \\
\left(\mathrm{cm}^{-3}\right)\end{array}$ & $\begin{array}{c}\text { EL2-like defect } \\
\text { concentration } \\
\left.(\times 10)^{-3} \mathrm{~cm}^{-3}\right)\end{array}$ & $\begin{array}{c}\text { Photo- } \\
\text { quenchable } \\
\text { fraction }(\%)\end{array}$ \\
\hline $1 \times 10^{16}$ & 2.4 & 86 \\
$1 \times 10^{17}$ & 2.7 & 85 \\
$1 \times 10^{18}$ & 1.8 & 84 \\
$1 \times 10^{19}$ & 1.4 & 56 \\
\hline \hline
\end{tabular}




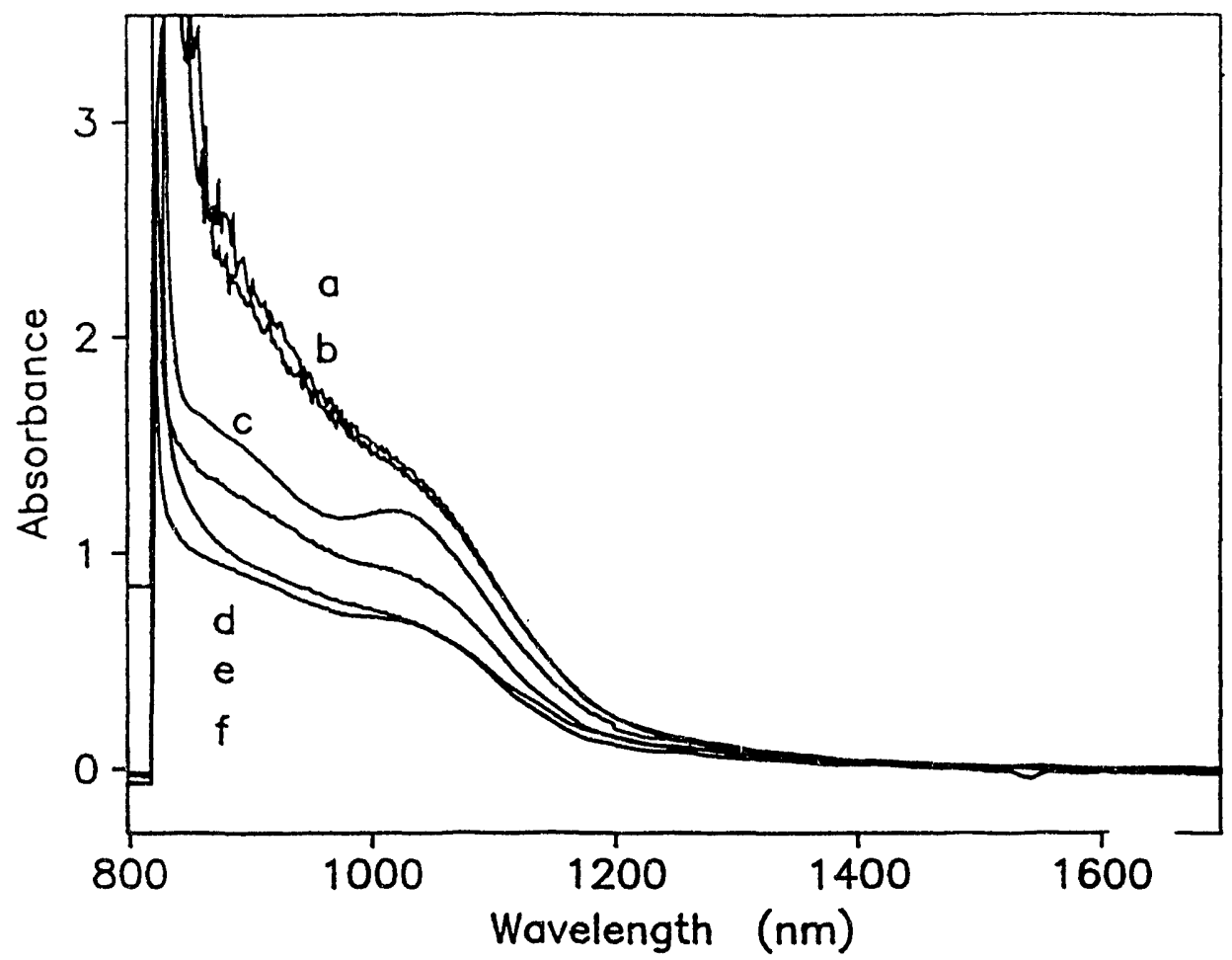

FIG. 2.10 IR absorption spectra of LTMBE GaAs:Si layers corrected for substrate absorption. (a) $10^{17} \mathrm{~cm}^{-3}$ doped layer, (b) $10^{16} \mathrm{~cm}^{3}$, (c) $10^{18} \mathrm{~cm}^{-3}$, (d) $10^{19} \mathrm{~cm}^{-3}$, (e) $10^{18} \mathrm{~cm}^{-3}$ doped layer after $30 \mathrm{~min}$. white light illumination, (f) $\mathrm{PQ}$ absorption of $10^{17} \mathrm{~cm}^{-3}$ doped layer given by the ratio of spectra taken before and after illumination.

The differences in the absorption spectra between LTMBE and LEC GaAs could possibly be due to othei defects or it could be broadening of the EL2 lineshape. Just because a GaAs material shows a strong absorption before the gap does not mean that it is necessarily due to EL2. Many deep level defects give rise to a similar absorption and distinguishing between them by shape alone can be difficult. Generally the accepted way of distinguishing between EL2 and other deep level defects is to check the photoquenching properties. If it is photoquenchable then it is due to EL2. Even this technique is not perfect though. If by pumping EL2 to 
the metastable state the Fermi level is changed then the charge state of other defects can be changed persistently until the normal state of EL2 is recovered. Such changes in charge state could incorrectly be perceived as metastability. This is not likely to be the case in the Si doped material, however. If any of the $\mathrm{Si}$ is active and acts as a shallow donor, then most of the AsGa-related should be occupied and in the neutral charge state (EPR measurements, discussed in section 2.3, indicate this to be true). Therefore pumping it to the metastable state should not change the charge state of it or any other defects. The shape of the PQ portion of the the spectra is the same as the NPQ portion of the spectra (see Fig. 2.10). The concentration of AsGa-related defects determined from IR absorption of the Si-doped layers is summarized in Table 2.5.

It is further justified assigning the IR absorption to EL2-like defects if we compare the amount of absorption to the concentration of ASGa-related defects required to account for the nonstoichiometry observed by PIXE. Table 2.6 lists the concentration of EL2-like defects determined by IR absorption and the according concentration of AsGa-related defects for the observed non-

TABLE 2.5 Concentration of EL20-like defect in LTMBE GaAs:Si.based on IR absorption measurements. $P Q$ fraction is determined after $30 \mathrm{~min}$. white light illumination.

\begin{tabular}{ccc}
\hline \hline $\begin{array}{c}S i \\
\text { concentration } \\
\left(\mathrm{cm}^{-3}\right)\end{array}$ & $\begin{array}{c}\text { EL2-like defect } \\
\text { concentration } \\
\left(\times 10^{19} \mathrm{~cm}^{-3}\right)\end{array}$ & $\begin{array}{c}\text { Photo- } \\
\text { quenchable } \\
\text { fraction }(\%)\end{array}$ \\
\hline $1 \times 10^{16}$ & 5.4 & 70 \\
$1 \times 10^{17}$ & 4.2 & 56 \\
$1 \times 10^{18}$ & 12.0 & 51 \\
$1 \times 10^{19}$ & 11.0 & 46 \\
\hline \hline
\end{tabular}


TABLE 2.6 Comparison of the concentration of AsGa-related defects in LTMBE GaAs based on IR absorption measurements and the equivalent concentration corresponding to the non-stoichiometry measured by PIXE.

\begin{tabular}{|c|c|c|}
\hline \multirow{2}{*}{$\begin{array}{l}\text { Sample doping } \\
\left(\mathrm{cm}^{-3}\right)\end{array}$} & \multicolumn{2}{|c|}{$\begin{array}{l}\text { AsGa-related defect } \\
\text { concentration }\left(\times 10^{19} \mathrm{~cm}^{-3}\right)\end{array}$} \\
\hline & IR absorption & PIXE equivalent \\
\hline undoped & 5 & $8( \pm 4)$ \\
\hline [Be] $10^{17}$ & 3 & 3 \\
\hline [Be] $10^{19}$ & $2^{*}$ & 2 \\
\hline [Si] 1017 & 7 & 3 \\
\hline [Si] $10^{19}$ & 11 & 19 \\
\hline totals & 28 & 35 \\
\hline
\end{tabular}

stoichiometry. The observed concentration of EL2-like defects nearly accounts for all the non-stoichiometry. The statistical error of the PIXE measurement is on the order of the EL2-like defect concentration of the Be doped samples, $4 \times 10^{19} \mathrm{~cm}^{-3}$. Adding up the columns of Table 2.6, we see that the EL2-like defects account for $80 \%$ of the excess As non-stoichiometry. This seems quite reasonable and gives us confidence in the value of the cross section used $\left(\sigma=10^{-16} \mathrm{~cm}^{2}\right)$ for the calibration of the IR absorption. It is also reasonable to expect that the remaining $20 \%$ of the excess As is in the form of other point defects such as $V_{G a}$ and $A s$ I or possibly small As clusters also.

The implicaticn is that the presence of $\mathrm{Si}$, or more importantly the presence of a higher concentration of AsGa-related defects in the Si doped samples, broadens the EL2-like absorption peak and reduces the metastability. It has been suggested that neighboring defects

\footnotetext{
- Total EL2-like defect concentration, corrected for compensation by $10^{19} \mathrm{~cm}^{-3} \mathrm{Be}$ acceptors.
} 
can disrupt the metastability of EL2-like defects. The metastability two neighboring defects could be disrupted through the interaction of strain fields or interaction of electronic energy states. In the first case a neighboring defect could cause the other defect to favor one direction as a lattice relaxation direction. If there is no energetic barrier along this direction then there can be no metastability. In the second case, as two AsGa-related defects are brought nearer together the electronic states can begin to interact. It is known that the metastability of EL2 occurs through an excited electronic state also know as an intra-center transition. If the excited state is perturbed through the interaction with the neighboring defect, then metastability can also be lost. These brief descriptions are not meant as definitive explanations for the elimination of metastability but rather are given as suggestive examples for how the presence of neighboring defects could conceivably disrupt metastability.

We will only consider for now the possibility of other AsGarelated defects disrupting the metastability since AsGa exist in the highest concentration of any defect. Consider now the probability an AsGa-related defect will have a neighbor within some specified distance. $t$ This is the case when not all the available Ga sublattice sites are occupied by a Ga atom,

$$
P_{\text {n.n. }} \text { (AsGa) }=1-P_{\text {all }}\left(G_{a G a}\right) \text {, }
$$

t After working out this problem I discovered a general treatment of the distribution of nearest neighbors in a random distribution of particles worked out by Chandrasekhar (1943). He notes that the problem was first considered by Hertz (1909). 
where $P_{\text {all }}\left(G_{G_{a}}\right)$ is the probability that all the sites are occupied by Ga atoms. That probability is

$$
P_{\text {all }}\left(G_{G a}\right)=\left[P\left(G_{a G a}\right)\right]^{N} \text {, }
$$

where $N$ is the total number of Ga sublatice sites within the specified distance. In terms of the concentration of AsGa, the probably a particular site is occupied by an $\mathrm{As}$ or $\mathrm{Ga}$ atom is respectively,

$$
\begin{aligned}
& P\left(A s_{G a}\right)=\frac{\left[A s_{G a}\right]}{2.2 \times 10^{22}}, \\
& P\left(G_{a_{a}}\right)=1-P\left(A s_{G a}\right) .
\end{aligned}
$$

Substituting eq's. 2.10, 2.9, and 2.8 back into 2.7 the expression for the probability an AsGa has a neighbor within a specified distance becomes,

$$
P_{n . n .}\left(A s_{G a}\right)=1-\left(1-\frac{\left[A s_{G a}\right]}{2.2 \times 10^{22}}\right)^{N}
$$

If we specify the distance in terms of nearest neighbor separations on the $\mathrm{Ga}$ sublattice (this would be next nearest neighibors of the diamond lattice) then the number of sites within that nearest neighbor shell can be counted. Values for $\mathrm{N}$ are summarized in Table 2.7. The number of sites for the third nearest neighbor shell was 
TABLE 2.7 A summary of the number of Ga sublattice sites within the sphere of specified nearest neighbor spacings.

\begin{tabular}{cc}
\hline $\begin{array}{c}\text { n.n. distance on the } \\
\text { Ga sublattice }\end{array}$ & \# of sites \\
\hline 1 & 12 \\
2 & 54 \\
3 & $\sim 160$ \\
\hline
\end{tabular}

estimated by calculating the number of sites within a sphere of radius of $3 / \sqrt{2} a_{0}$, with 4 sites per $\left(a_{0}\right)^{3}$. A plot of the probability of encountering a neighboring $A S G a$ is shown in Fig. 2.11 for the first 3 nearest neighbor separations. Small probabilities are proportional to the concentration of defects yielding a straight line while higher probabilities tend to saturate. Also plotted in Fig. 2.11 is the NPQ

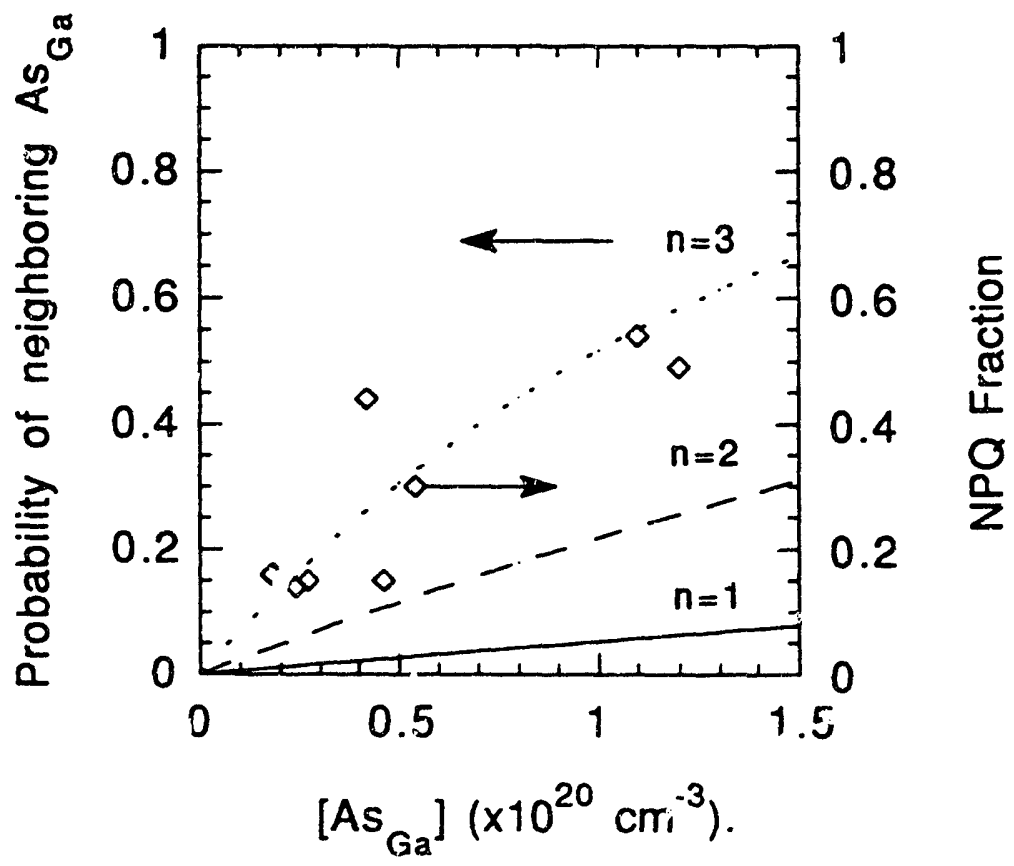

FIG. 2.11 Dual plot of the non-photoquenchable fraction of El2-like defects vs. the EL2-like defect concentration $(0)$ and the probability of a neighboring defect within a specified nearest neighbor separation:

$\longrightarrow$ n.n. $=1, \ldots-n . r_{1}=2, \cdots n . n .=3$. 
fraction of defects of all the $\mathrm{Be}-, \mathrm{Si}-$ and undoped layers as a function of AsGa-related defect concentration. If we assume the defects are incorporated randomly in the crystal, then it appears that a defect can influence the metastability of its neighbor at a distance of approximately 3 nearest neighbor separations on the $\mathrm{Ga}$ sublattice. This corresponds to a defect separation of $12 \AA$.

In collaboration with Dr. Krambrock at the University of Paderborn in Germany, optically detected magnetic resonance experiments (ODMR) were performed on these samples (Krambrock, et al. 1992). ODMR measurements are more sensitive than standard EPR measurements to defects with a large spin-lattice coupling, such as a defect with a neighbor. The neighboring defect breaks the local symmetry of the crystal, easing the selection rules for coupling to phonons, hence increasing the spin-lattice relaxation. These measurements find AsGa-related defects with multiple environments confirming the presence of other defects near antisites.

The IR absorption spectra will continue to be interpreted in terms of an EL2-like defect, particularly the $P Q$ portion of the absorption. The similarities in properties between the AsGa-related defects in LTMBE GaAs and EL2 outnumber the differences. No evidence so far has conclusively led to its interpretation as another defect.

\subsubsection{Electrical properties}

One striking aspect of electrical measurements of the doped LTMBE GaAs layers is the lack of strong $n$ - or p-type conductivity. Neither the shallow acceptor Be, nor the shallow donor Si supplies 
free carriers to its respective band. For the Be doped layers, Fig. 2.12 shows results of variable temperature Hall effect and resistivity measurements for the as-grown sample doped with $[\mathrm{Be}]=$ $10^{16} \mathrm{~cm}^{-3}$. Unexpectedly, both this and the sample doped with $[\mathrm{Be}]=$ $1017 \mathrm{~cm}^{-3}$ exhibit $n$-type conductivity. Unfortunately, in the two more highly doped samples $\left([\mathrm{Be}]=10^{18}\right.$ and $10^{19} \mathrm{~cm}^{-3}$ ), about $10 \mathrm{~nm}$ of standard GaAs were inadvertently Be doped. After growing the undoped standard GaAs, the Be shutter was opened before the substrate had fully cooled to the lower growth temperature. The $\mathrm{Be}$ incorporated during growth at the elevated temperature was electrically active. This created a shunting layer at the interface and made the study of the LTMBE layers with electrical measurements impossible. It did not, however, affect optical

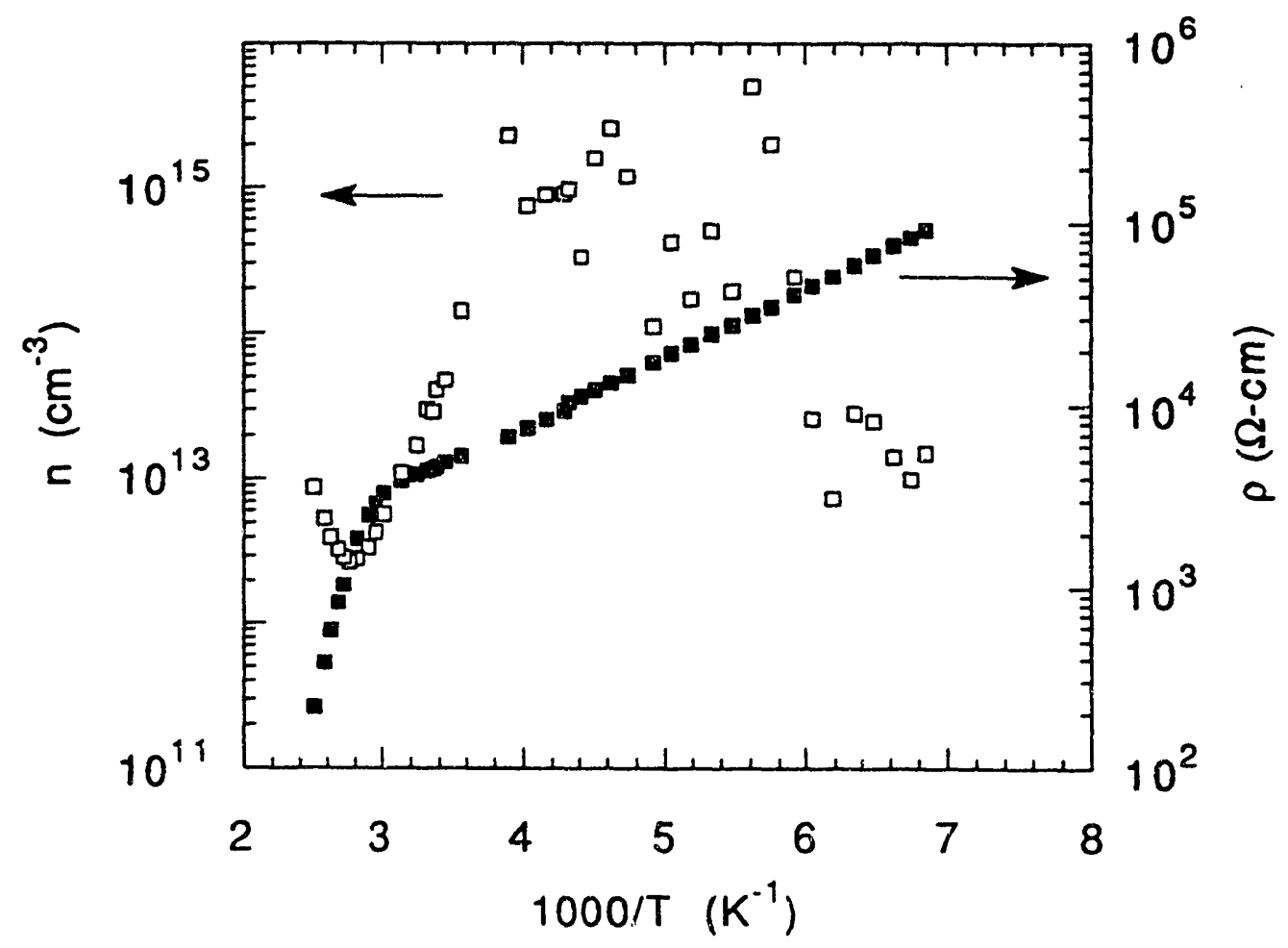

FIG. 2.12 Plot of variable temperature Hall effect and resistivity for a Be doped (1016 $\left.\mathrm{crn}^{-3}\right)$ LTMBE GaAs layer. 


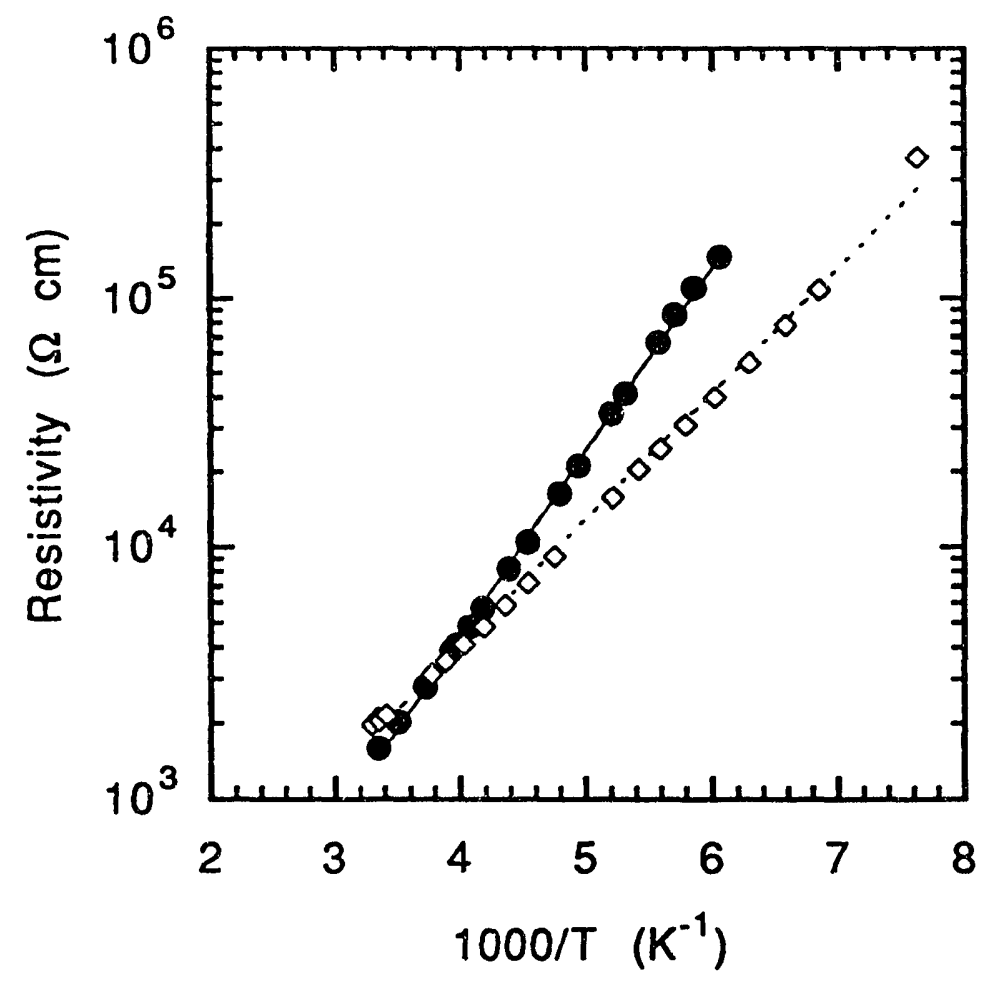

FIG. 2.13 Plot of variable temperature resistivity data for LTMBE GaAs layers: $\Delta$ - undoped, $-[\mathrm{Si}]=10^{16} \mathrm{~cm}^{-3}$. Solid line is exponential fit to resistivity data.

measurements of these layers.

Results of variable temperature resistivity measurements on the Si doped LTMBE GaAs layers are shown in Fig's. 2.12 and 2.13. Again, the conductivity is n-type but not strongly. It is surprising that the Si dupped layers are not highly conductive. Si is a shallow donor. One would expect that the AsGa-related deep donors would not be able to compensate the shallow donor levels and that strong $n$ type conductivity should be observed. The lack of electrical activity implies that either,

1. the dopants do not occupy substitutional sites but rather sit interstitially, 
2. the $\mathrm{Be}(\mathrm{Si})$ is compensated by donor- (acceptor-) like defects and is unable to supply holes (electrons) to the valence (conduction) band,

3. the dopants are passivated by a nearby defect forming a defect-impurity complex which has a deep level or is electrically inactive.

Similar to the undoped layers, the conductivity of the doped material is dominated by a hopping mechanism. Electrical conduction occurs by the hopping of carriers via the deep-defect levels indicating the Fermi level is pinned at midgap. Like the undoped sample, the conductivity of the doped layers can be fit using eq. 2.6 to determine the activation energy $\varepsilon_{3}$ for hopping. Table 2.8

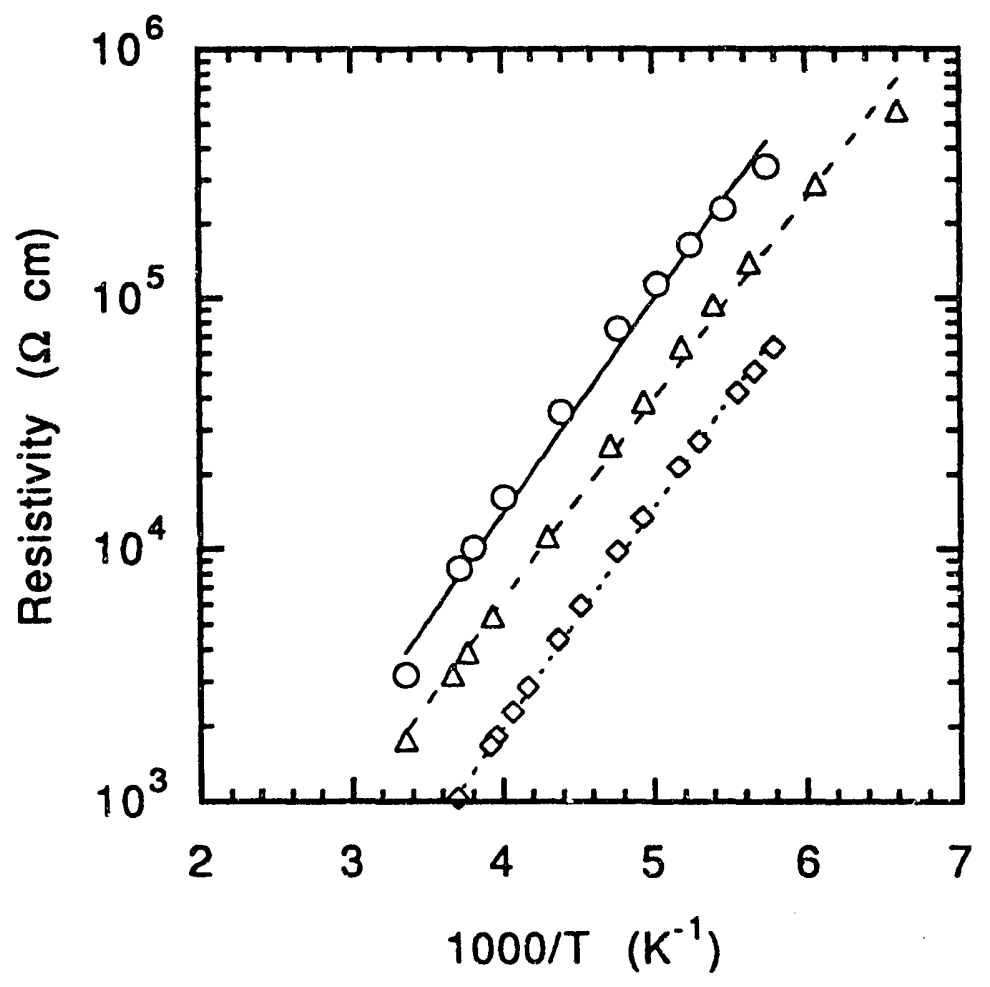

FIG. 2.14 Arrhenius plot of the resistivity for LTMBE GaAs layers: $0-[\mathrm{Si}]=$ $10^{19} \mathrm{~cm}^{-3}, \Delta-[\mathrm{Si}]=10^{18} \mathrm{~cm}^{-3}, \Delta-[\mathrm{Si}]=10^{17} \mathrm{~cm}^{-3}$. Straight lines are exponential fits for determining the activation energy $\varepsilon_{3}$ summarized in Table 2.8 . 
summarizes the values of $\varepsilon_{3}$ determined for the undoped, $\mathrm{Be}$ and $\mathrm{Si}$ doped samples.

The width of the energy difference between the unperturbed hopping level and the Fermi level is described by $\varepsilon_{3}$ (see Fig. 2.7). Table 2.8 shows that $\varepsilon_{3}$ increases as the Be concentration is reduced or as the $\mathrm{Si}$ concentration is increased. This indicates that the Fermi level is moving higher in the hopping band and that possibly the width of the AsGa-related defect band is increasing also. In the latter case, the width of the AsGa-related defect band depends on the concentration of defects. A higher concentration of $A S_{a}$-related defects results in a wider band due to increased Coulombic interaction between the closer spaced centers. However, increasing the concentration of hopping centers while maintaining the same compensation ratio would result in a higher hopping probability due to the increased wave function overlap. Therefore the compensation ratio for the AsGa-related defect, defined as $\left[\mathrm{AsGa}^{+}\right] /\left[\mathrm{As} \mathrm{Ga}_{\mathrm{a}}\right]$, must be decreasing also. IR absorption measurements indicate a significantly higher concentration of AsGa only in the $10^{18}$ and $10^{19} \mathrm{~cm}^{-3} \mathrm{Si}$ doped samples while $\varepsilon_{3}$ is larger for all $\mathrm{Si}$ doped samples. Since $\varepsilon_{3}$ does not depend strongly on the concentration of AsGa related defects, this indicates the Fermi level must be higher up in the defect band of the Si doped layers. A small fraction of electrically active $\mathrm{Si}$ could be responsible for moving $E_{F}$ higher in the defect band. Conversely, the Be wili compensate the AsGa-related donors, moving $E_{F}$ lower in the defect band and reducing $\varepsilon_{3}$. 
TABLE 2.8 A summary of the electrical conductivity activation energies for undoped, Be doped and Si doped LTMBE GaAs.

\begin{tabular}{cc}
\hline $\begin{array}{c}\text { Sample Doping } \\
\left(\mathrm{cm}^{-3}\right)\end{array}$ & $\begin{array}{c}\varepsilon_{3} \\
(\mathrm{meV})\end{array}$ \\
\hline Undoped & 99 \\
Be $10^{16}$ & 75 \\
Be $10^{17}$ & 92 \\
Si $10^{16}$ & 150 \\
Si $10^{17}$ & 157 \\
Si $10^{18}$ & 162 \\
Si $10^{19}$ & 157 \\
\hline \hline
\end{tabular}

Figure 2.15 plots the resistivity versus $\mathrm{T}^{-1 / 4}$ of the layers doped $[\mathrm{Si}]=10^{17}, 10^{18}$ and $10^{19} \mathrm{~cm}^{-3}$. A straight line fits the $T^{-1 / 4}$ plot of the data better than for the Arhenius plot indicating a Mott type of variable range hopping. The temperature dependence of the resistivity can be expressed as (Mott 1968)

$$
\rho(T)=\rho_{0} \exp \left[\left(\frac{T_{0}}{T}\right)^{1 / 4}\right],
$$

where,

$$
T_{0}=\frac{\beta}{k g\left(E_{f}\right) a^{3}},
$$

$g\left(E_{f}\right)$ is the density of states at the Fermi level, a the localization radius of states near the Fermi level, and $\beta=21.2$ is a numerical coefficient which can be derived from percolation theory (Shklovskii and Efros 1984, pg. 206). The value of $T_{0}$ does not represent any real temperature (300 million Kelvin is quite hot!) but rather yields information about the density of states at the Fermi level $g\left(E_{f}\right)$ and 
also the average hopping length $\bar{r} \approx a\left(T_{0} / T\right)^{1 / 4}$. The values of $T_{0}$ determined from fits to the data and the derived values for $g\left(E_{f}\right)$ and $\bar{r}$ for a temperature of $200 \mathrm{~K}$ are summarized in Table 2.9. A value of $a=8 \AA$ was used for the localization radius. This value is supported by our annealing results in Chapter 3 and is supported by other researchers (Kaminska and Weber 1990, Look, et al. 1990). Values for $\bar{r}$ of approximately $300 \AA$ suggest that variable range hopping is occurring between quite distant defects, on the order of 10-15 defects away.

For hopping to occur, unoccupied levels must exist. The carrier then hops from its occupied level to the nearby unoccupied level. The probability of a hop is proportional to two quantities: 1. the wave function overlap between states which depends exponentially on the separation, and 2. the energy difference between states. For closely spaced hopping centers the energy separation is larger due to increased coulombic interactions. Thus at low temperatures, if the energy separation between neighboring centers is too large, hopping to more distant centers can become more probable even though the state function overlap is less. Maximizing the hopping probability at

TABLE 2.9 Summary of variable range hopping parameters determined for $\mathrm{Si}$ doped LTMBE GaAs. $T_{0}$ determined from a fit of eq. 2.12 to the resistivity data shown in Fig. 2.14. $g\left(E_{f}\right)$ is the density of states at the Fermi level. $\bar{r} 200 \mathrm{~K}$ is the average hopping length at $T=200 \mathrm{~K}$.

\begin{tabular}{cccc}
\hline $\begin{array}{c}\text { Si Doping } \\
\left(\mathrm{cm}^{-3}\right)\end{array}$ & $\begin{array}{c}T_{0} \\
\left(\times 10^{8} \mathrm{~K}\right)\end{array}$ & $\begin{array}{c}g\left(E_{f}\right) \\
\left(\times 10^{18} \mathrm{~cm}^{-3} \mathrm{eV}^{-1}\right)\end{array}$ & $\bar{r}_{200 \mathrm{~K}(\AA)}$ \\
\hline $10^{17}$ & 3.13 & 1.50 & 283 \\
$10^{18}$ & 3.84 & 1.25 & 298 \\
$10^{19}$ & 3.42 & 1.40 & 289 \\
\hline \hline
\end{tabular}




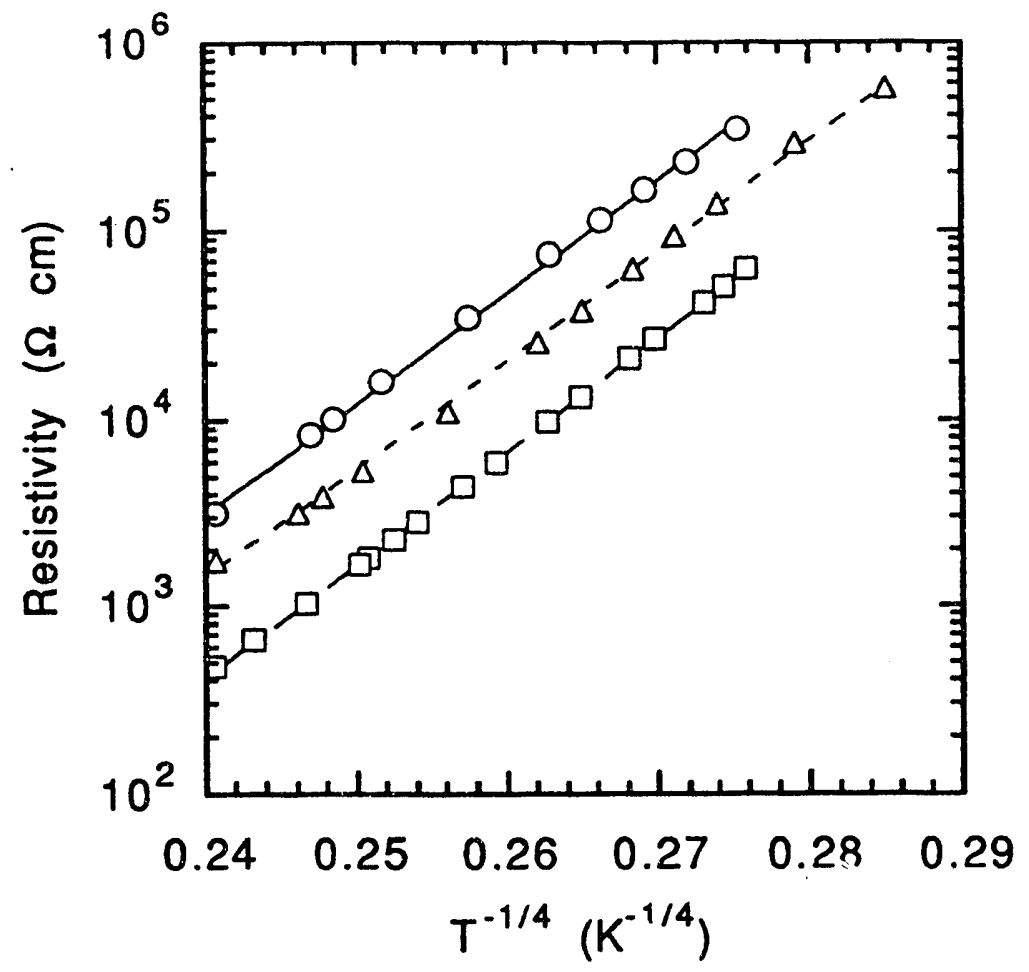

FIG. 2.15 Logarithmic plot of resistivity vs. T-1/4 for LTMBE GaAs layers:

$0-[\mathrm{Si}]=10^{19} \mathrm{~cm}^{-3}, \square-[\mathrm{Si}]=10^{18} \mathrm{~cm}^{-3}, \Delta-[\mathrm{Si}]=10^{17} \mathrm{~cm}^{-3}$. Straight lines are exponential fits indicating a Mott $\mathrm{T}^{-1 / 4}$ type of hopping mechanism.

a particular temperature yields an ideal energy band for hopping to occur. This ideal energy band is a function of temperature and results in :11e $T-1 / 4$ dependence of the activation energy (Shklovskii and Efros 1984, pg. 202). This can be thought of as a temperature dependent activation energy which decreases with decreasing temperature.

\subsubsection{Local Vibrational Modes}

It is well known that when a lighter impurity atom occupies the site of a heavier host atoin, it can oscillate at a frequency higher than the highest frequency phonons (LO, TO at $\Gamma$ ). These vibrations are localized to the impurity atom and do not propagate 
through the crystal. Hence, they are referred to as local vibrational modes (LVM's). LVM's give rise to absorption and Raman peaks in the mid IR. To address the issue of whether the Be impurities occupy substitutional or interstitial sites in as-grown layers, IR absorption and Raman measurements of the Be LVM were made on the LTMBE layers.

First, an attempt to measure the LVM by IR absorption was made. The reported LVM frequency for Be in GaAs is $482 \mathrm{~cm}^{-1}$ (Laithwaite, et al. 1975). Usually, when measuring the LVM of a shallow impurity species like $\mathrm{Be}$, the sample must be electron irradiation damaged to remove free carriers which absorb in this range of the infrared. Otherwise the free carrier absorption would mask the LVM. It seemed fortunate that the Be was not electrically active in LTMBE layers making irradiation unnecessary. Unfortunately, even if the $\mathrm{Be}$ atoms were substitutional, the LVM absorption from $3 \mu \mathrm{m}$ of the $10^{19} \mathrm{~cm}^{-3}$ doped material is near the detection limit.

Rarnan spectroscopy of LVM's can overcome the limitation of IR absorption which requires relatively thick samples containing a high concentration of shallow impurities. Also the need to compensate samples containing a high concentration of free carriers is alleviated This is because the depth of the probing light is reduced to the near surface region. The frobe depth, $d=1 / 2 \alpha$, where $\alpha$ is the absorption coefficient of the light (Sturge 1968), can be $500 \AA$ for a photon energy of $2.54 \mathrm{eV}$ in the case of an argon ion laser shining on GaAs $\left(\alpha=10^{5} \mathrm{~cm}^{-1}\right)$. The free carrier absorption over this thickness is negligible. Limitations of the Raman technique are 
that the spectral resolution of Raman is somewhat poorer than IR absorption, $2 \mathrm{~cm}^{-1}$ as compared to $.05 \mathrm{~cm}^{-1}$ and determination of the concentration from the scattered intensity is more complicated. Intensities must be compared to the strength of the nearby multiphonon peak to account for variations in the laser power, detector geometry, etc., but even then the scattering cross sections depend on the incident phonon energy. Resonance effects occur whereby the strength of phonon bands or the LVM can be greatly increased when the optical excitation matches the $E_{1}$ gap. This is known as resonant Raman scattering (Martin and Falicov 1983). Resonant enhancement of the Raman scattering can be used advantageously to increase sensitivity, but an absolute determination of the concentration becomes difficult.

This Raman study of the Be LVM was performed with the expert assistance of Dr. Joel Ager at LBL. A backscattering arrangement was used at room temperature. The Ar ion laser excitation energy was $2.54 \mathrm{eV}$ for a penetration depth of $500 \AA$. The polarization of the incident and scattered light was parallel to the [011] direction. This configuration gave a maximum LVM signal. Crossed polarizers in the $<001>$ directions would completely suppress the nearby multiphonon peak (2TO) but would have also cut down the LVM intensity.

Figure 2.16 shows the Raman spectrum of the as-grown LTMBE GaAs doped $10^{19} \mathrm{~cm}^{-3}$ with Be. A well resolved LVM peak is visible at $478 \mathrm{~cm}^{-1}$. The reported Raman LVM frequency is $482 \mathrm{~cm}^{-1}$ at 77K (Wagner, et al. 1980). Since our measurement was performed at room temperature, some softening of the mode is to be expected. Noise in the system sets a lower detection limit for $\mathrm{Be}$ at roughly 


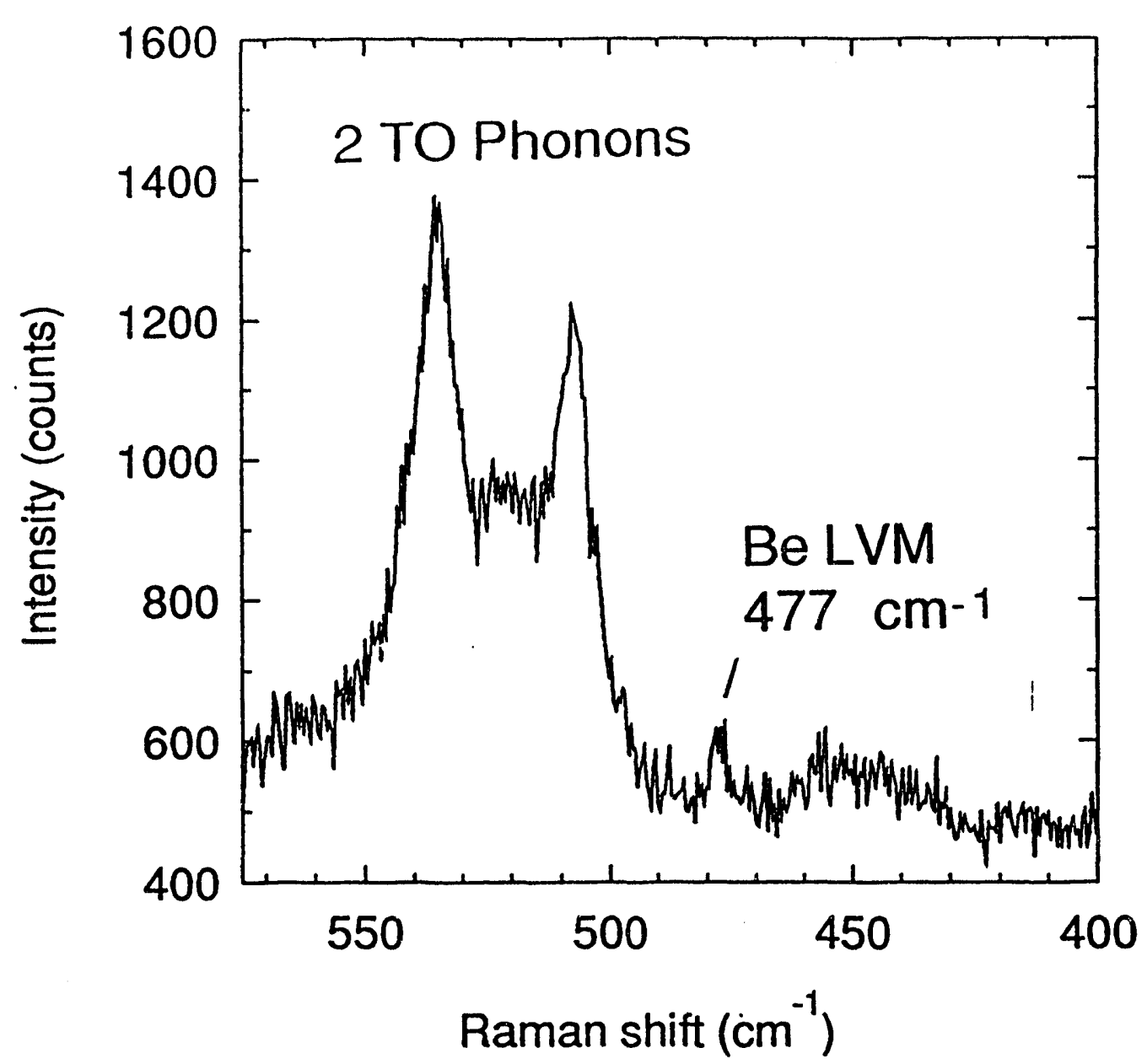

XBL 919-1921

FIG. 2.16 Raman spectrum of LTMBE GaAs:Be $10^{19} \mathrm{~cm}^{-3}$ taken at room temperature. The Be LVM is visible at $477 \mathrm{~cm}^{-1}$ to the right of the large 2TO phonon band.

$3 \times 10^{18} \mathrm{~cm}^{-3}$ using the calibration of Wagner et al. (1980). Our results indicate that a significant fraction of the total $\mathrm{Be}$, greater than $50 \%$, must occupy substitutional positions. Therefore, the $\mathrm{Be}$ must either be compensated by native defects or form complexes with them. The possibility of forming Be-defect complexes will be considered further in chapter 3 in the discussion of annealing results. 
Raman measurements were also performed to look for the $\mathrm{Si}$ LVM. The Si on Ga site LVM is reported at $384 \mathrm{~cm}^{-1}$. No Si LVM Raman scattering could be detected in the $10^{19} \mathrm{~cm}^{-3}$ doped sample even though SIMS analysis shows that the $\mathrm{Si}$ is incorporated in the layer at the intended concentrations. Similar observations have been made Manasreh et al. (1990) They measured the Si LVM by IR absorption as a function of growth temperature. They found that the Si LVM decreases in intensity as the growth temperature is lowered below $400^{\circ} \mathrm{C}$ and disappears completely below $250^{\circ} \mathrm{C}$. The fact that Si does not occupy substitutional sites explains the lack of strong n-type conductivity. The question then arises, how is the $\mathrm{Si}$ incorporated in the layers. What site does the Si actually occupy? Presently it is unknown. It would be interesting to know if the $\mathrm{Si}$ occupies an interstitial site, forms complexes with other defects, or forms clusteis.

\subsubsection{Electron paramagnetic resonance}

In order to determine the compensation of the AsGa-related level, electron paramagnetic resonance (EPR) measurements were performed to find the quadruplet signal of the singly ionized $\mathrm{AsGa}^{+}$. To increase the sensitivity and reduce the background signal from the substrate, the substrate was lapped to a thickness of $\sim 100 \mu \mathrm{m}$. To increase the total number of spins in the microwave cavity four of the $3 \mu \mathrm{m}$ layers were stacked together. The noise equivalent $\mathrm{AsGa}^{+}$spin concentration for the volume of LTMBE material $\left(\sim 1 \mathrm{~cm}^{2} \times\right.$ $3 \mu \mathrm{m}$ ) used was $\sim 5 \times 10^{17} \mathrm{~cm}^{-3}$. The quadruplet could only be observed in the $10^{18}$ and $10^{19} \mathrm{~cm}^{-3} \mathrm{Be}$ doped samples. The quadruplet spectrum is shown in Fig. 2.17. No temperature or 

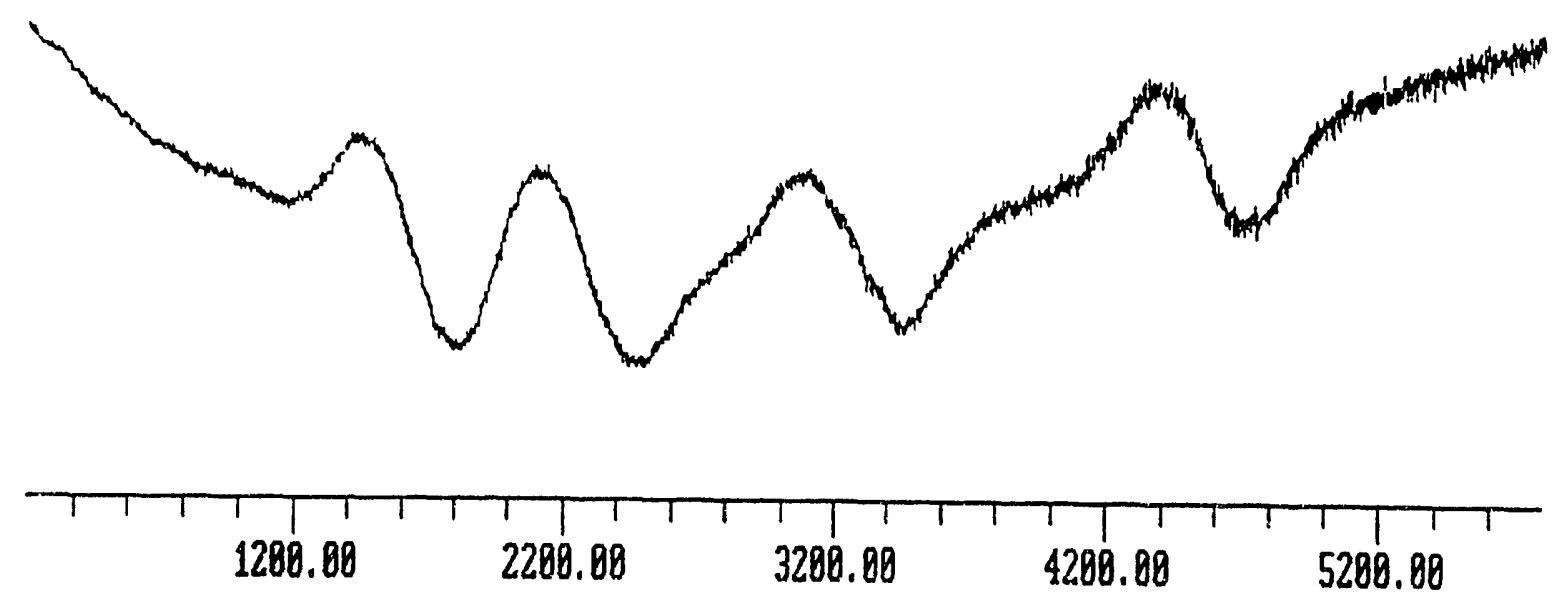

Magnetic field (Gauss)

FIG. 2.17 EPR spectrum of the LTMBE GaAs:Be $10^{19} \mathrm{~cm}^{-3}$ showing quadruplet signal characteristic of $\mathrm{AsGa}^{+}$. Measurement temperature is $\sim 12 \mathrm{~K}$.

microwave power saturation of the EPR signal were observed, consistent with previous reports for $\mathrm{ASGa}^{+}$signals in LTMBE GaAs.

The number of spins was calibrated against a known Si:P standard. Details of determining the spin concentration can be found in the test by Poole (1983). The measured concentration of spins was the same as the Be doping within experimental error, $1.2 \times 10^{18}$ and $1.0 \times 10^{19} \mathrm{~cm}^{-3}$ for the respective samples.

The implication of this result is that native defects do not compensate the the AsGa-related midgap level to any significant degree. For the Si-doped and undoped layers the compensation of the midgap level can not be detected and must therefore be below $1 \%$. For the layers doped $[\mathrm{Si}]=10^{18}$ and $10^{19} \mathrm{~cm}^{-3}$ the compensation must be less than $0.25 \%$. Only when $B e$ acceptors are introduced in a high concentration is the AsGa-related level compensated. 
Consider the gallium vacancy. Even though positron annihilation experiments suggest a concentration of negatively charged $\mathrm{VGa}^{-}$on the order of $10^{19} \mathrm{~cm}^{-3}$, they are not compensating the main deep donor defect. Several possible explanation for the lack of compensation by $V_{G a}$ exist. First the vacancies could be forming divacancy or other multiple vacancy complexes. The electronic levels of the divacancy are unknown but could be completely different than the isolated vacancy. Another explanation is that the vacancy can relax into another configuration which is donor-like. A defect which can act both as an acceptor or a donor depending upon its configuration is known as an amphoteric defect. This would be similar to the amphoteric nature of $\mathrm{Si}$ in GaAs which can be a donor on a Ga site, SiGa, and an acceptor on the As site, SiAs.

Theoretical predictions have indicated that an isolated $V_{G}$ a configuration should be a triple acceptor. The o/- transition is calculated to be in the lower half of the bandgap at an energy of $E_{v}+$

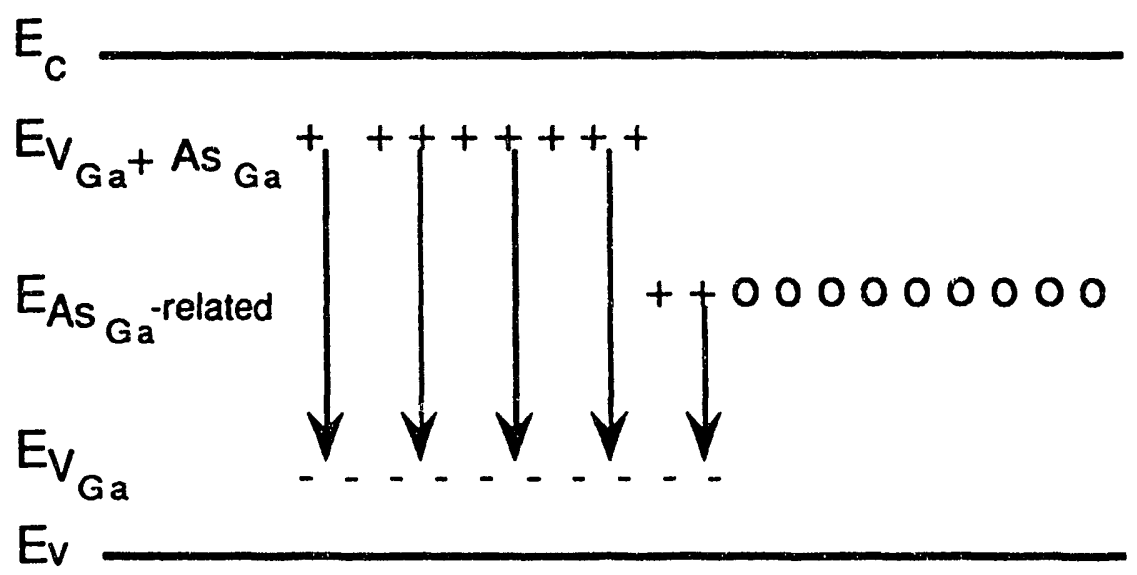

FIG. 2.18 Schematic energy diagram illustrating the amphoteric nature of a $V_{G a}$ defect resulting in the compensation of a relaxed $A s_{G a}-V_{G a}$ complex and not the AsGa midgap level. 
$300 \mathrm{meV}$ (Baraff and Schlüter 1985). A second configuration was predicted in which a neighboring $A s$ atom can relax towards the $V_{G a}$ to form a defect resembling a $V_{A s}-A s G a$ complex. Theoretical calculations of the electronic structure of the $V_{A s}-A s G$ a configuration predict a donor-like level in the upper half of the bandgap (Baraff and Schlüter 1986). It has been shown recently by Walukiewicz that under equilibrium conditions, concentrations of the two defects should be formed such that the Fermi level is stabilized near midgap (Walukiewicz 1989). Equal concentrations of donor and acceptor like configurations of the defect should compensate each other, as illustrated in Fig. 2.18, leaving the AsGarelated midgap level occupied by electrons. Thus the high concentration of $V_{G a}$ can contribute to trapping positrons without compensating the midgap level.

Positron annihilation

The results of slow positron annihilation experiments on the Be doped samples are shown in Fig. 2.19. The $S(E)$ response is shown for samples doped with $[\mathrm{Be}]=10^{16}$ and $10^{19} \mathrm{~cm}^{-3}$. The undoped sample is included as a reference. The solid lines represent the best fit of equation 2.3 to the $S(E)$ response. The parameters of that fit are tabulated in Table 2.10. We see that both the bulk $S$ parameter, $S_{b}$, and the diffusion length $L$ are suppressed somewhat in the layers containing $\mathrm{Be}$. At first glance, these two results give conflicting indications about the concentration of excess $V_{G a}$ but this can be resolved. A reduced $S_{b}$ indicates a lower $\left[V_{G a}\right]$ while the reduced $L$ apparently indicates an increased $\left[V_{G a}\right] . S_{b}$ is a more reliable indicator of the presence of $V_{G a}$. The reason being that our 
estimation of $\Delta\left[V_{G a}\right]$ from $L$ assumes the same positron diffusivity for all samples which might not be true. Analogous to an electron the positron diffusivity is,

$$
D_{+}=\frac{k_{B} T}{m^{\star}} \tau
$$

where $m^{*}$ is the positron effective mass and $\tau$ is the positron scattering time. The presence of a high concentration of $\mathrm{Be}$ should decrease the diffusivity by increasing the rate of ionized impurity scattering. Thus $D_{+}$is not a constant and the $\Delta\left[\mathrm{V}_{\mathrm{G} a}\right]$ will be

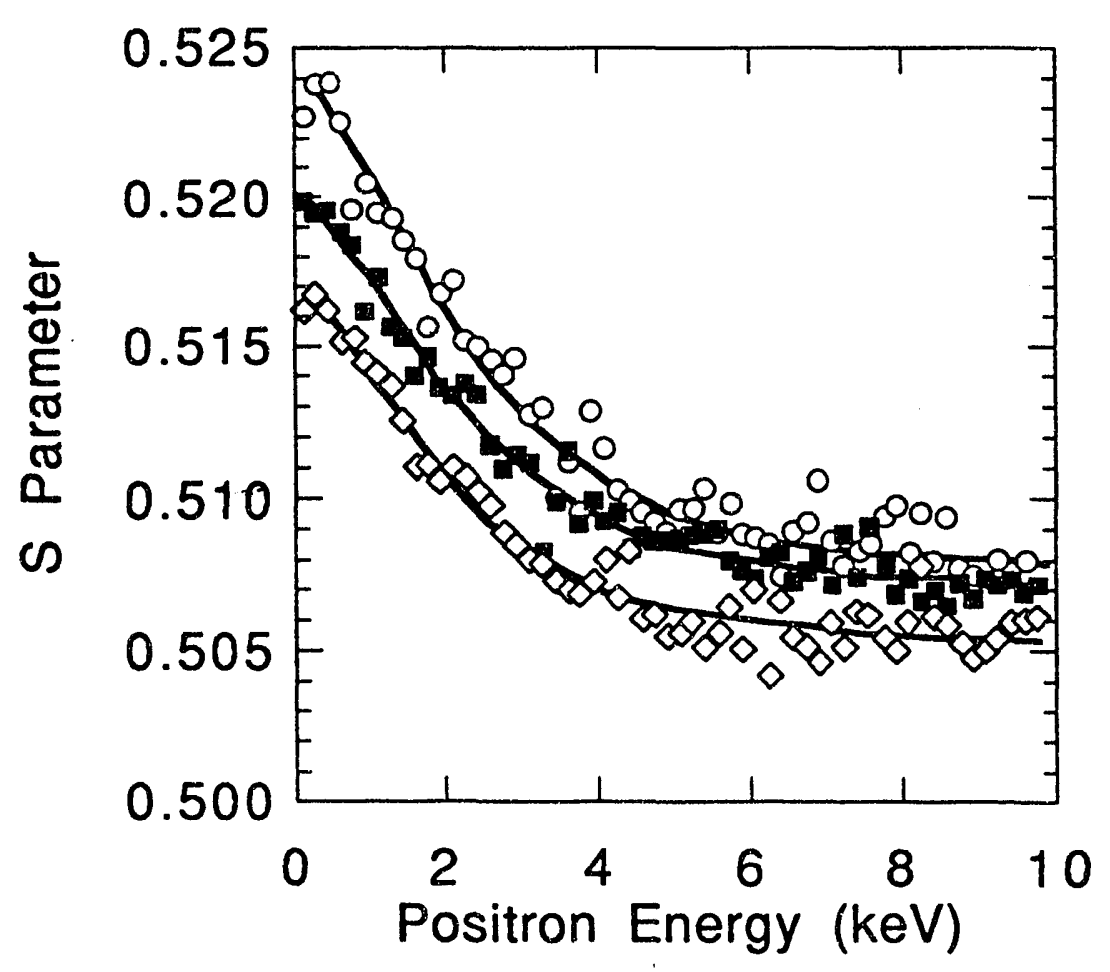

FIG. 2.19 Results of slow positron annihilation investigations of Be doped LTMBE GaAs layers. The S-parameter is plotted vs. the positron implantation energy. (0) undoped LTMBE GaAs, (घ) LTMBE GaAs:Be $10^{16} \mathrm{~cm}^{-3},(\diamond)$

LTMBE GaAs:Be $10^{16} \mathrm{~cm}^{-3}$. The solid lines are the best fit to the data using eq. 2.3. 


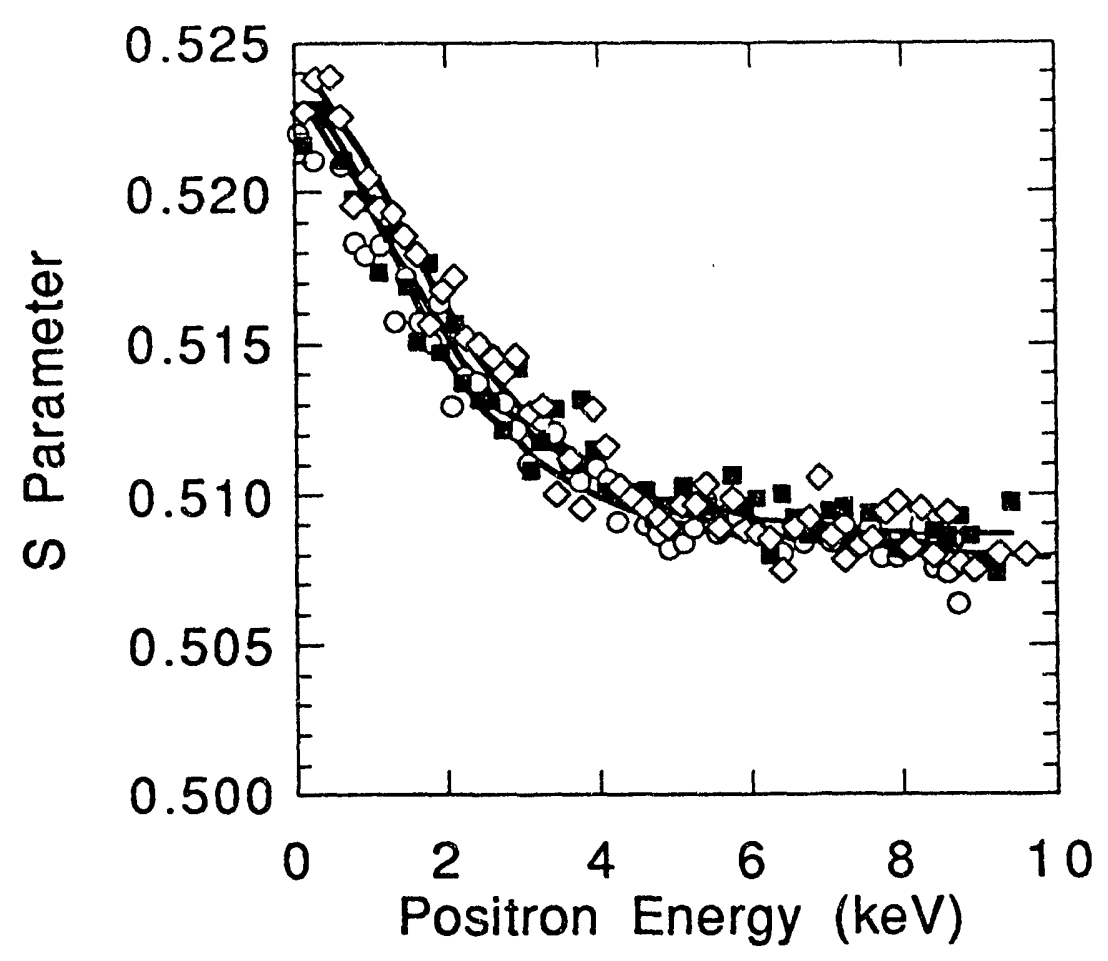

FIG. 2.20 Results of slow positron annihilation investigations of Si doped LTMBE GaAs layers. The S-parameter is plotted vs. the positron implantation energy. ( $\diamond)$ undoped LTMBE GaAs, (a) LTMBE GaAs:Si $1017 \mathrm{~cm}^{-3}$, (0)

LTMBE GaAs:Be $10^{19} \mathrm{~cm}^{-3}$. The solid lines are the best fit to the data using eq. 2.3.

overestimated for the heavily Be doped layers.

Thus, $S_{b}$ indicates a reduction of the $\left[\mathrm{V}_{\mathrm{Ga}}\right.$ ] in the $\mathrm{Be}$ doped layers as compared to the undoped layers. However, $S_{b}$ is still greater than for the LEC substrate indicating excess $V_{G a}$ are still incorporated in the $\mathrm{Be}$ doped layers. The fact that $\mathrm{Be}$ suppresses the $V_{G a}$ concentration in the LTMBE layer is not surprising. This trend is understandable as one expects lower concentrations of negatively charged [ $\mathrm{V}_{\mathrm{Ga}}$ ] in the presence of $\mathrm{Be}$ acceptors.

The results of slow positron annihilation experiments on the Si doped LTMBE layers are shown in Fig. 2.20. The $S(E)$ response of 
TABLE 2.10 Summary of the fitted parameters for slow positron annihilation indicating the bulk $S$ parameter, $S_{b}$, the surface $S$ parameter, $S_{S}$, the diffusion length, $L$, and the excess concentration of $V_{G a}$ derived from $L$.

\begin{tabular}{lcccc}
\hline \hline \multicolumn{1}{c}{ Sample } & $S_{b}$ & $S_{S}$ & $L(\AA)$ & $\begin{array}{c}\Delta\left[\mathrm{VGa}_{\mathrm{Ga}}\right. \\
\times 10^{19}\left(\mathrm{~cm}^{-3}\right)\end{array}$ \\
\hline LEC GaAs & 0.50618 & 0.51654 & 361 & $\ldots$ \\
LTMBE GaAs: & & & & \\
\cline { 1 - 1 } Undoped & 0.50748 & 0.52419 & 317 & 1.18 \\
Be $10^{16}\left(\mathrm{~cm}^{-3}\right)$ & 0.50690 & 0.51980 & 318 & 1.15 \\
Be $10^{19}\left(\mathrm{~cm}^{-3}\right)$ & 0.50508 & 0.51688 & 277 & 2.78 \\
Si $10^{17}\left(\mathrm{~cm}^{-3}\right)$ & 0.50836 & 0.52290 & 263 & 3.53 \\
Si $10^{19}\left(\mathrm{~cm}^{-3}\right)$ & 0.50785 & 0.52368 & 231 & 5.75 \\
\hline \hline
\end{tabular}

the undoped layer is included for comparison. The data, including scatter, appear to fall on top of each other indicating the concentration of $V_{G a}$ is the approximately the same in all cases. Analysis of the curves by fitting to eq. 2.3 is summarized in Table 2.10. Values obtained for $L$ would indicate, however, that the concentration of $V_{G a}$ increases with $S i$ doping. The values of $S_{b}$, though, are the same within error indicating the same concentration of $\mathrm{VGa}_{\mathrm{G}}$. Although $S_{b}$ can be a better indicator of the $V_{G}$ a concentration, it does tend to saturate at high vacancy concentrations. I would also hesitate to over analyze values obtained for the $L$ parameter in deriving $V_{G a}$ concentrations. In that derivation, parameters are assumed to be 'jonstant which may not be and unknown values derived from theory are used.

In summary of the positron annihilation results, all the LTMBE GaAs layers contain an excess concentration of $V_{G a}$ compared to bulk 
grown LEC GaAs. The concentration of excess $V_{G a}$ is on the order of $10^{19} \mathrm{~cm}^{-3}$. The concentration of $V_{G a}$ is higher in the $\mathrm{Si}$ doped layers than the Be doped layer. It is possible that the $[S i]=10^{19} \mathrm{~cm}^{-3}$ doped sample contains the highest concentration of $V_{G a}$ but this has not been shown conclusively.

\subsubsection{Constant photocurrent characterization}

In an effort to determine if other defect levels exist in LTMBE GaAs besides the dominant AsGa-related level at midgap, constant photocurrent spectra were taken (Grimmeiss and Ledebo 1975). As the name of the technique implies, the current generated across a photoconductive sample by illumination is kept constant as the wavelength is scanned. The intensity of the illumination is adjusted to maintain a steady state current. The quasi-Fermi level is fixed for a given current across a semiconductor. This means that the occupation of defect levels is also fixed so that transients due to the charging and discharging of levels can be avoided. Thus, interpretation of spectra is more simple and the spectra can be taken more quickly than if a constant intensity is used.

To show how this technique works consider a single deep level of energy $E_{T 1}$, plus a shallow level in the upper half of the bandgap as shown in Fig. 2.21. Under steady state conditions and for photon energies $h v<E_{T 1}-E_{v}$ detailed balance yields,

$$
I(h v) \sigma_{n 1}^{o}(h v) n_{T 1}-c_{T 1}\left(N_{T 1}-n_{T 1}\right) n=0
$$


where $I(h v)$ is the intensity of the exciting light, $\sigma_{n l}^{o}(h v)$ is the optical cross section for the exsitation of an electron from the deep level to the conduction band, $n_{T l}$ is the density of deep levels occupied by electrons, $c_{T 1}$ is the sum of the thermal and optical capture rates for electrons by the deep level, $N_{T 1}$ is the concentration of the deep level, and $n$ is the concentration of free electrons in the conduction band. By adjusting the intensity $I(h v)$ the steady-state photocurrent can be kept constant at all photon energies. A fixed photocurrent means a constant density of free electrons and a constant occupancy of the impurity level. From eq. 2.15 it follows that

$$
\sigma_{n 1}^{o}(h v) \alpha \frac{1}{I(h v)}
$$

Thus by plotting the inverse of the intensity of light to maintain a constant photocurrent versus the photon energy, the spectral dependence of the photoionization cross section is obtained. For

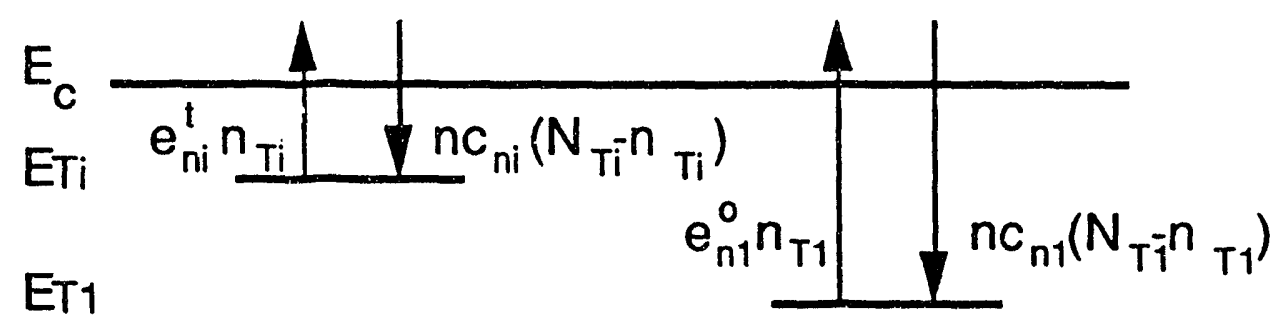

Ev

FIG. 2.21 Carrier generation and recombination via a deep donor level with binding energy $E_{T 1}$ and a shallow donor level. The temperature is such that thermal emission dominates the shallow emission and optical emission dominate the deep level emission. 
multiple deep levels the spectral dependence is the sum so that individual photoionization cross sections are superimposed on each other.

Constant photocurrent measurements are analogous to photocapacitive techniques. However, they are useful for material containing a high concentration of defects which would otherwise have too large a leakage current for photocapacitive measurements. The constant photocurrent technique has found useful applications to characterize amorphous and polycrystalline materials for photovoltaic solar energy conversion (Santos, et al. 1991).

Measurements of our LTMBE layers were performed at Xerox PARC with the assistance of Dr. Paulo Santos. Figure 2.22 shows the logarithm of the inverse photon intensity in arbitrary units versus photon energy $(\mathrm{eV})$ of several doped LTMBE GaAs layers and a SI-LEC substrate for comparison. In all cases, except for the sample doped with $[\mathrm{Be}]=10^{19} \mathrm{~cm}^{-3}$, we see an onset for photoionization at 0.73 $\mathrm{eV}$. For the SI-LEC sample this is just the excitation of electrons from EL2 to the conduction band. For the LTMBE layers we will call it the excitation of electrons from the neutral AsGa-related defect level to the conduction band. Note however, the details of the excitation above $0.73 \mathrm{eV}$ differ depending on the doping of the layer. It is not clear that a second photoionization cross section exists in any of the samples. Thus, we can only confirm the presence of the As Ga-related electronic level at midgap. The lack of an ionization level which might correspond to an isolated $V_{G a}$ suggests that the vacancies might form divacancies or complexes. However this deduction is by no means conclusive. 
The $10^{19} \mathrm{~cm}^{-3} \mathrm{Be}$ doped sample appears unique compared to the other samples. No photocurrent onset is seen at $0.73 \mathrm{eV}$. Only light having an energy equal to or greater than the bandgap has an effect on the photocurrent. A possible explanation for this effect is that the occupation of the AsGa-related deep donor is changed significantly by the $10^{19} \mathrm{~cm}^{-3} \mathrm{Be}$ acceptors. Capture processes dominate due to the decreased occupation of the deep level so we do not see the photogenerated carriers as a current. This effect was not studied further. The primary purpose of the constant photocurrent measurements was to find other defect levels in the gap. We could not find any other defect ionization levels so these measurements were not pursued further. 


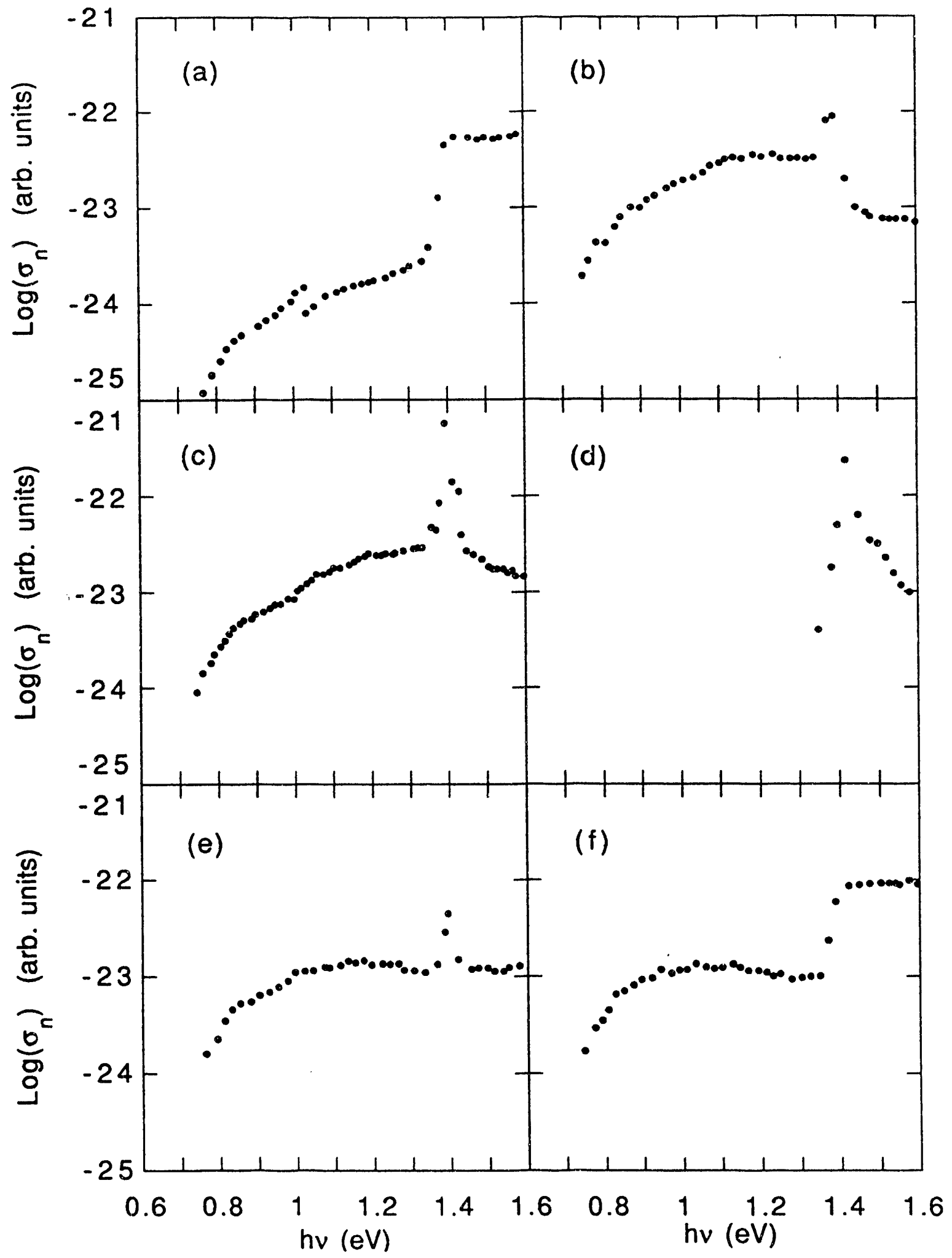

FIG. 2.22 Spectral dependence of the logarithm of the inverse light intensity while maintaining a steady-state photocurrent in LTMBE GaAs. The inverse intensity is proportional to the photoionization cross section. LTMBE GaAs samples are: (a) SI-LEC substrate for comparison, (b) undoped, (c) $[\mathrm{Be}]=10^{16}$ $\mathrm{cm}^{-3}$, (c) $[\mathrm{Be}]=10^{19} \mathrm{~cm}^{-3}$, (c) $[\mathrm{Si}]=10^{16} \mathrm{~cm}^{-3}$, (c) $[\mathrm{Si}]=10^{19} \mathrm{~cm}^{-3}$. 


\subsubsection{Photoluminescence}

Photoluminescence has proven to be very useful for the assessment of impurities and native defects in semiconductors. Energy levels can be detected at concentrations as low as about $1015 \mathrm{~cm}^{-3}$. Carriers are excited across the gap with a powerful laser and the light re-emitted due to the recombination of excited carriers is analyzed. Much like DLTS the presence of certain defects and impurities can be determined quickly by recognizing the spectral signatures in luminescence. It should be pointed out that lack of a peak does not imply that a level is not present in tho sample. All recombination pathways, whether luminescent or not, are competing in parallel. The lack of one particular peak might mean that another peak or mechanism is out-competing it, and not that the level is not present. Probably the largest unsolved problem of PL is the role of nonradiative recombination. There is no doubt that it is extremely important since all III-V compounds show a considerable enhancement of luminescence intensity upon being cooled. Nonradiative recombination centers certainly exist in LTMBE GaAs and are responsible for the lack of luminescence in $200^{\circ} \mathrm{C}$ grown material. For a review of the theory and application of PL to GaAs see the review by Bebb and Williams (Bebb and Williams 1971, Williams and Bebb 1971).

PL measurements were made on our LTMBE GaAs:Be layers looking particularly for peaks near the band edge associated with the $\mathrm{Be}$ and lower energy peaks which might be associated with recombinations to deep levels. 


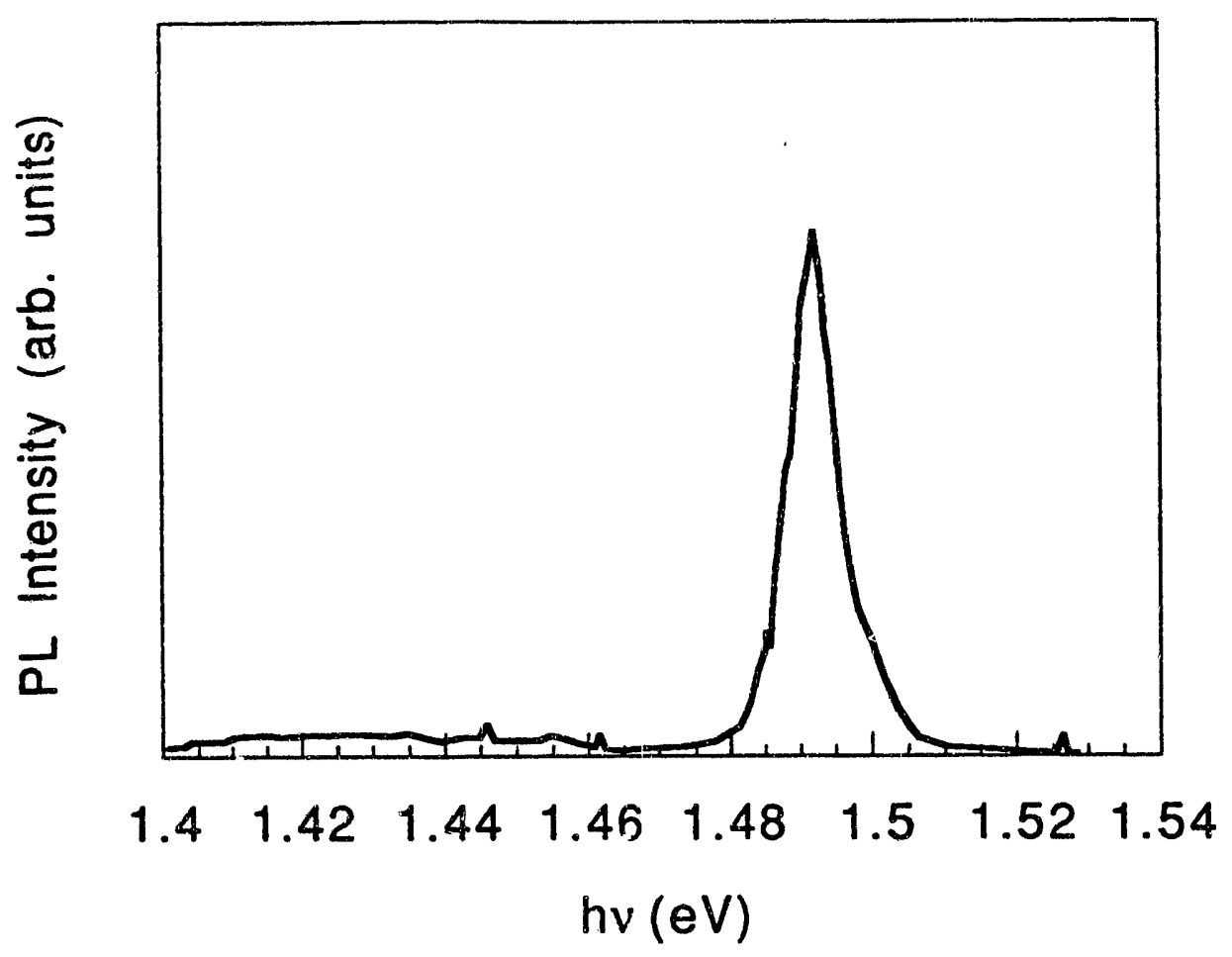

FIG. 2.23 Spectral dependence of the relative photoluminescence intensity in LTMBE GaAs:Be $10^{16} \mathrm{~cm}^{3}$. The peak at 1.492 is assigned to a $\left(\mathrm{Be}^{\circ}, \mathrm{e}\right)$ recombination and is typical for all the Be doped samples.

PL measurements were performed by Prof. Nancy Haegel at UCLA. The system was comprised of an Ar ion laser operating at 514 $\mathrm{nm}(2.41 \mathrm{eV})$ at $100 \mathrm{~mW}$ of power, a liquid He cryostat for low temperature $(T=4.2 \mathrm{~K}$ ) operation, a double grating monochromator and a Ge IR detector.

The results of PL measurements on the $1016 \mathrm{Be}$ doped samples are shown in Fig. 2.23. This spectrum is typical of the other $\mathrm{Be}$ doped samples. A peak at $1.4920 \mathrm{eV}$ is clearly visible. It corresponds to the recombination of an electron in the conduction band with a neutral acceptor. Subtracting the binding energy of a hole to a Be acceptor $(0.028 \mathrm{eV})$ from the energy of the bandgap $(1.5177 \mathrm{eV})$ the recombination energy is calculated to be $1.490 \mathrm{eV}$. 
TABLE 2.11 A summary of the relative $\left(\mathrm{Be}^{\circ}, \mathrm{e}\right) \mathrm{PL}$ intensity for $\mathrm{Be}$ doped LTMBE GaAs layers.

\begin{tabular}{cc}
\hline $\begin{array}{c}{[\mathrm{Be}]} \\
\left(\mathrm{cm}^{-3}\right)\end{array}$ & $\begin{array}{c}\text { Ge Detector Output } \\
(\mathrm{meV})\end{array}$ \\
\hline $10^{16}$ & 1.5 \\
$10^{17}$ & 2.5 \\
$10^{18}$ & 3.2 \\
$10^{19}$ & 4.7 \\
\hline
\end{tabular}

Given the linewidth of the peak $(\sim 10 \mathrm{meV})$ and the system resolution $(\sim 0.5 \mathrm{meV})$ the agreement is quite good. No exiton lines are seen, but they are not expected at $4.2 \mathrm{~K}$. The samples must be cooled further since the binding energy of exitons is approximately $1.5 \mathrm{meV}$ in GaAs.

Table 2.11 summarizes the relative intensity of the $1.492 \mathrm{eV}$ peak. Note that the intensity depends only slightly on the $\mathrm{Be}$ concentration, increasing approximately logarithmically with increasing $\mathrm{Be}$ concentration. The lack of a dependence of the PL intensity on $\mathrm{Be}$ concentration is not understood at this time.

\subsection{Summary and Concluding Comments}

This section will summarize and discuss what has been learned about defects and doping of as-grown LTMBE GaAs. The picture of the interplay between defects and impurities should be clearer when separated from the presentation of the characterization techniques.

The growth of GaAs at $300^{\circ} \mathrm{C}$ results in the incorporation of $0.5 \%$ excess As as measure by PIXE. This was not enough excess As to cause a measurable expansion of the lattice. The excess As is 
incorporated in the as-grown layers as a high concentration of point defects. The main defects identified are related to $\mathrm{ASG}_{\mathrm{a}}$ and to a lesser extent $V_{G a}$. The concentration of neutral $A s_{G a}{ }^{\circ}$-related defects as measured by IR absorption is on the order of $3 \times 10^{19}$ $\mathrm{cm}^{-3}$. No singly charged $\mathrm{AsGa}^{+}$could be detected by EPR. If they do exist it would be at a concentration below the detection limit of $5 \times 10^{17} \mathrm{~cm}^{-3}$. Similar to EL2, a large fraction, roughly $85 \%$, of the AsGa ${ }^{0}$-related defects can be photoquenched. The thermal recovery of the metastable state occurs around 130K. However, no ZPL at $1196 \mathrm{~nm}$ could be seen in the LTMBE layers. A possible explanation for the lack of an observable ZPL is that inhomogeneous strain in the layer and the presence of nearby defects broadens the ZPL peak.

Evidence for the existence of $V_{G a}$ on the order of $10^{19} \mathrm{~cm}^{-3}$ was found by slow positron annihilation studies. Although it is generally accepted that $V_{G a}$ has an acceptor level in the lower half of the bandgap, no compensation of the AsGa-related level could be measured. Possible explanations are that the $V_{G a}$ form clusters or, after Walukiewicz (1989), the $V_{G a}$ are amphoteric. Since defect charge neutrality tends to stabilize the Fermi level at midgap, the acceptor- and donor-like configurations of the $V_{G a}$ would tend to compensate one another. Or perhaps, $V_{G a}$ does not have an electrically active level in the bandgap. Even though theorists have calculated an acceptor level for $V_{G a}$, no concrete experimental evidence has been found to prove its existence.

The electrical conductivity of LTMBE layers is dominated by the hopping of electrons over the deep donor defect level. We found a nearest neighbor hopping type of behavior for the undoped layers as 
opposed to variable range hopping as reported by other researchers (Kaminska and Weber 1990, Look, et al. 1990). Nearest neighbor hopping is indicated by the straight exponential dependence of the conductivity on inverse temperature. No T-1/4 behavior which would be indicative of variable range hopping was observed.

Perhaps the most striking aspect of the electrical properties is that intentionally introduced ciopants do not generate any extrinsic free carriers. It was possible to introduce both $\mathrm{Be}$ and $\mathrm{Si}$ at a high concentration of $10^{19} \mathrm{~cm}^{-3}$ and the Fermi level remained pinned at midgap. Underlying the lack of free carriers is a fundamental difference between the $\mathrm{Be}$ and $\mathrm{Si}$ in the as-grown layers. The $\mathrm{Be}$ occupies a substitutional site as shown by Raman spectroscopy while the Si does not. It is seen by EPR that the Be does compensate the AsGa-related level, making it paramagnetic. The $\mathrm{Si}$ on the other hand appears electrically inactive. If the $\mathrm{Si}$ occupied an isolated substitutional $\mathrm{Ga}$ site, it should generate free electrons in the conduction band. It would not be possible for the AsGa-related deep donor level to compensate the shallow donor. No LVM could be detected either. It is not likely that the Si forms clusters. The low temperature growth limits the surface mobility. Instead, the Si either occupies an interstitial site or forms an unknown complex.

The behavior of $\mathrm{Si}$ and $\mathrm{Be}$ is different in regard to their influence on the native defects as well. Si drastically enhances the incorporation of excess As into the layers mainly in the form of AsGa-related defects. The Si appears to be very efficient at binding extra As: Five times more excess As, from $4 \times 10^{19} \mathrm{~cm}^{-3}$ to $2 \times 10^{20}$ 
$\mathrm{cm}^{-3}$ AsGa-related defects, is incorporated in the layer by the addition of Si. This corresponds to over 10 extra AsGa per additional $\mathrm{Si}$ atom. Be on the other hand tends to suppress the $V_{G}$ a concentration while having no effect on the total concentration of AsGa-related defects. At first glance the enhancement of the deep donor defect concentration by $\mathrm{Si}$ appears contrary the thermodynamics; an intentionally introduced donor should suppress the formation of donor-like defects. However the $\mathrm{Si}$ is not substitutional and should not influence the thermodynamic equilibrium as a shallow donor. Instead, the enhancement of the AsGa-related defect concentration is probably related to the effect of the Si on the surface during growth. One possible mechanism is that the $\mathrm{Si}$ catalyzes the breakup of arsenic tetramers arriving at the surface thereby incoporating more arsenic atoms. A second possible mechanism is the reduction of the diffusivity of ad-atoms on the surface. $\mathrm{Si}$ is a slow diffuser in the bulk and therefore a slow diffuser on the surface. Already the surface mobility of $\mathrm{Ga}$ and As atoms is limited by the low temperature growth and many defects are formed. With $\mathrm{Si}$ additionally limiting the surface mobility even more defects should be introduced into the growing layer.

The effect of $\mathrm{Be}$ on the suppression of the $\mathrm{V}_{\mathrm{Ga}}$ concentration can be understood in terms of thermodynamic arguments. That is, if the $V_{G a}$ can be considered to be an acceptor also. Charge neutrality and the law of mass action would imply that the intentional introduction of an acceptor should suppress the formation of acceptor-like defects in favor of donor-like defects and should have no effect on electrically inactive defects. This would be an 
argument in favor of an amphoteric model of the $\mathrm{V}_{\mathrm{Ga}}$. Addition of $\mathrm{Be}$ would drive the formation of $V_{G a}$ towards the donor-like configuration. Thus, a positively charged donor-like $V_{G a}$ would not be detected since it can not trap positrons.

This explanation of the interplay between principle native defects and shallow impurities forms a cohesive story of the experimental results of as-grown LTMBE GaAs layers. An interesting piece of future work would be to determine the atomic configuration of the $\mathrm{Si}$ is incorporated and to find the mechanism responsible for the enhancement of the $A s_{G a}$-related defect concentration.

\section{References}

Baj, M. and P. Dreszer. "EL2 Intracenter Absorption under Hydrostatic Pressure." Mat. Science Forum 38-41, 101 (1989).

Baraff, G.A. and M. Schlüter. Phys. Rev. Lett. 55, 1327 (1985).

Baraff, G.A. and M. Schlüter. Phys. Rev. B 33, 7346 (1986).

Bebb, H.B. and E.W. Williams, "Photoluminescence I: Theory." R.K. Williardson and A.C. Beer, ed. (Academic Press, New York, 1971) pp. 181.

Chandrasekhar, S. "Stochastic problems in physics and Astronomy." Rev. Modern Phys. 15, 1 (1943).

Coates, R. and E.W.J. Mitchell. Advan. in Phys. 24, 593 (1975).

Dlubek, G. and R. Kruse. "Positron Studies of Defects in III-V Semiconductor Compounds." Phys Stat. Sol. (a) 102, 443 (1987).

Grimmeiss, H.G. and L.-Å. Ledebo. "Spectral distribution of photoionization cross sections by photoconductivity measurements." J. Appl. Phys. 46, 2155 (1975).

Hertz, P. Math. Ann. 67, 387 (1909). 
Hoinkis, M. and E.R. Weber. "Differentiation of Electron-ParamagneticResonance signals of arsenic antisite defects in GaAs." Phys. Rev. B40, 3872 (1989).

Jackson, W.B. and C.C. Tsai. "Hydrogen transport in amorphous silicon." Phys. Rev. 845, 6564 (1992).

Johnson, N.M., R.D. Burnham, R.A. Street and R.L. Thornton. Phys. Rev. B 33, 1102 (1986).

Kaminska, M. (1991).

Kaminska, M., Z. Liliental-Weber, E.R. Weber, T. George, J.B. Kortright, F.W. Smith, B.-Y. Tsaur and A.R. Calawa. "Structural properties of As-rich GaAs grown by molecular beam epitaxy at low temperatures." Appl Phys. Lett. 54,1881 (1989).

Kaminska, M. and E.R. Weber, "Hopping conduction of low temperature GaAs within an arsenic antisite defect band." E.M. Anastassakis and J.D. Joannopouloss, ed., 20th International Conference on the Physics of Semiconductors (ICPS) (World Scientific, Thessaloniki, Greece, 1990) vol. 1, pp. 473.

Kato, Y., T. Shimada, Y. Shiraki and K.F. Komatsubara. J. Appl. Phys. 45, 104 (1974).

Krambrock, K., M. Linde, J.M. Spaeth, D.C. Look, D.E. Bliss and W. Walukiewicz. "Arsenic antisite-related defects in low-temperature grown GaAs." Semicond. Sci. Technol. 7, 1037 (1992).

Lagowski, J., M. Kaminska, J.M. Parsey Jr., H.C. Gatos and M. Lichtensteiger. "Passivation of the dominant deep level (EL2) in GaAs by hydrogen." Appl. Phys. Lett. 41, 1078 (1982).

Laithwaite, K., R.C. Newman and P.D. Greene. J. Phys. C: Solid St. Phys. 8, L77 (1975).

Lee, J.-L., A. Uedono and S. Tanigawa. J. Appl. Phys. 67, 6153 (1990).

Lee, J.-L., L. Wei, S. Tanigawa, T. Nakagawa, K. Ohta and J.Y. Lee. "The effects of point defects on the electrical activation of Si-implanted GaAs during rapid thermal annealing." IEEE Trans. Electron Devices 39, 176 (1992).

Lee, J.-L., L. Wei, S. Tanigawa, H. Oigawa and Y. Nannichi. "The effects of surface oxides on the creation of point defects in GaAs studied by slow positron beam." Jap. J. Appl. Phys. 30, L138 (1990). 
Look, D.C., D.C. Walters, M.O. Manasreh, J.R. Sizelove, S. C.E. and K.R. Evans. "Anomalous Hall-effect results in low-temperature molecular-beam-epitaxial GaAs: Hopping in a dense EL2-like band." Phys. Rev. B42, 3578 (1990).

Manasreh, M.O., D.C. Look, K.R. Evans and C.E. Stutz. "Infrared absorption of deep defects in molecular-beam-epitaxial GaAs layers grown at $200^{\circ} \mathrm{C}$ : Observation of an EL2-like defect." Phys. Rev B. 41, 10272 (1990).

Martin, G.M. "Optical assessment of the main electron trap in bulk semiinsulating GaAs." Appl. Phys. Lett. 39, 747 (1981).

Martin, R.M. and L.M. Falicov, "Resonant Raman Scattering." M. Cardona, ed. (Springer-Verlag, New York, 1983) vol. 8, pp. 80.

Mott, N.F. "Conduction in glasses containing transition metal ions." J. Non-Cryst. Solids 1,1 (1968).

Paesler, M., S.C. Agarwal and R. Zallen. "Proceedings of the $13^{\text {th }}$ International Conference on Amorphous and Liquid Semiconductors." J. Non-Cryst. Solids 114, (1989).

Pearton, S.J., W.C. Dautremont-Smith, J. Chevallier, C.W. Tu and K.D. Cummings. "Hydrogenation of shallow-donor levels in GaAs." J. Appl. Phys. 59, 2821 (1986).

Poole, C.P.J., "Electron Spin Resonance, 2nd Ed., A comprehensive Treatise on Experimental Techniques." (John Wiley \& Sons, Inc., New York, 1983).

Puska, J.J., C. Corbel and R.M. Nieminen. Phys. Rev. B41, 9980 (1990).

Santos, P.V., C.F.D. Graef and I. Chamboulevron. "Light-induced metastability in A-Ge-H." J. Non-Cryst. Solids 128, 243 (1991).

Schulz, P.J. and K.G. Lynn. "Interaction of positron beams with surfaces, thin films, and interfaces." Rev. Mod. Phys. 60, 701 (1988).

Shklovskii, B.I. and A.L. Efros, "Electronic Properties of Doped Semiconductors." M. Cardona, Ed., (Springer-Verlag, Berlin, 1984).

Sturge, M.D. Phys. Rev. 127, 768 (1968).

Wagner, R.J., J.J. Krebs, G.H. Strauss and A.M. White. Solid State Commun. $36,15(1980)$.

Walukiewicz, W. "Amphoteric native defects in semiconductors." Appl. Phys. Lett. 54, 2094 (1989).

Williams, E.W. and H.B. Bebb, "Photoluminescence II: Gallium Arsenide." R.K. Williardson and A.C. Beer, ed. (Academic Press, New York, 1971) pp. 321. 
Yu, K.M. and Z. Liliental-Weber. "Lattice site locations of excess arsenic atoms in gallium arsenide grown by low-temperature molecular beam epitaxy." Appl. Phys. Lett. 59, 3267 (1991). 


\section{Chapter 3. Annealing Characteristics of LTMBE GaAs}

\subsection{Introduction}

In general, annealing studies of defects in semiconductors are important both technologically and scientifically. From the technological viewpoint, the fabrication of devices often requires heat treatments at elevated temperatures. Depending on the process, whether it is the diffusion of dopants, activation of implanted ions, oxidation or alloying of contact metals etc., temperatures can range from just above room temperature to the melting point of the material which can be above $1000{ }^{\circ} \mathrm{C}$. It is essential to understand the effect of the heat treatment on the entire materials system: substrate, active region, insulator and metallization in order to create a functioning device. Defects can influence the processes which occur during annealing in several ways. Defects can be created by the annealing and directly influence the electrical properties of the active layer. Defects can anneal away and alter the compensation scheme of the substrate causing the conductivity to change. In addition, defects can influence materials properties indirectly. A particular defect might act as a gettering site for impurities. Vacancy-like defects are known to enhance the diffusion. Doped regions can spread to larger volumes 
than intended due to the enhanced diffusion. The metals might diffuse into the active regions ruining the device. Therefore it is essential to understand the annealing characteristics of the defects in the material.

This chapter covers the results of annealing studies of the LTMBE GaAs layers. The results of electrical and optical measurements of doped and undoped layers annealed isochronally and isothermally will be presented and interpreted. A model for the annihilation of ASGa defects in terms of vacancy enhanced diffusion of the defects to precipitates is presented. Activation enthalpies for the migration of $A s G a$ are derived. The interaction of the defects with the dopants $\mathrm{Si}$ and $\mathrm{Be}$ will be considered in light of the precipitation.

\subsection{Isochronal annealing \\ 3.2.1. Annealing conditions}

Samples were isochronally annealed at $330,370,415,470$, $550,625,700$ and $800^{\circ} \mathrm{C}$ for 20 minutes each. For $550^{\circ} \mathrm{C}$ and below, the samples were annealed in flowing $\mathrm{N}_{2}$. Above $550^{\circ} \mathrm{C}$ the sample was sealed in an evacuated ampoule with enough elemental As to maintain an overpressure of 2 atm. at the annealing temperature to prevent As loss. The amount of As was estimated from the ideal gas law assuming $\mathrm{As}_{4}$ was the primary gaseous species. The As is stored in a nitrogen dry box to prevent oxidation. It is only removed just before annealing to be weighed and added to the ampoule. When sealing the ampoule, the LTMBE samples and As are placed at the far end of the ampoule away from the plug to minimize any heating from 
the hydrogen torch. After sealing the As and samples are moved to opposite ends of the ampoule to prevent any direct contact during the anneal. The sample end of the ampoule is placed in the furnace first so when the ampul is removed afterwards, the As condenses on the end of the ampoule away from the sample. After annealing, the ampoule is carefully cut with a diamond saw to break the vacuum. The samples are rinsed in $30 \% \mathrm{HF}$ to remove any As condensed on the surface. The backside of the substrate is polished for optical measurements.

\subsubsection{IR absorption}

IR absorption spectra were taken after each annealing step to observe the concentration of neutral AsGa-related defects. The IR absorption was measured at a photon energy of $1.24 \mathrm{eV}(\lambda=1 \mu \mathrm{m})$ versus annealing temperature and is shown in Fig. 3.1 and Fig. 3.2 for the $\mathrm{Be}$ and $\mathrm{Si}$ doped samples respectively. The filled-in symbols connected by solid lines represent the total neutral defect absorption taken at $10 \mathrm{~K}$ before illumination. The open symbols connected by dashed lines represent the photoquenchable portion of the absorption determined from the ratio of the absorption before and after illumination. The obvious feature in both Fig's. 3.1 and 3.2 is the distinct annealing stage between 470 and $550^{\circ} \mathrm{C}$ corresponding to a large decrease in the total IR absorption. Another feature of the data is that the non-photoquenchable (NPQ) AsGarelated defects start annealing away at lower temperatures than the $P Q$ defects. This is deduced from the fact that the total ( $P Q+N P Q)$ IR absorption decreases more than the photoquenchable portion for 


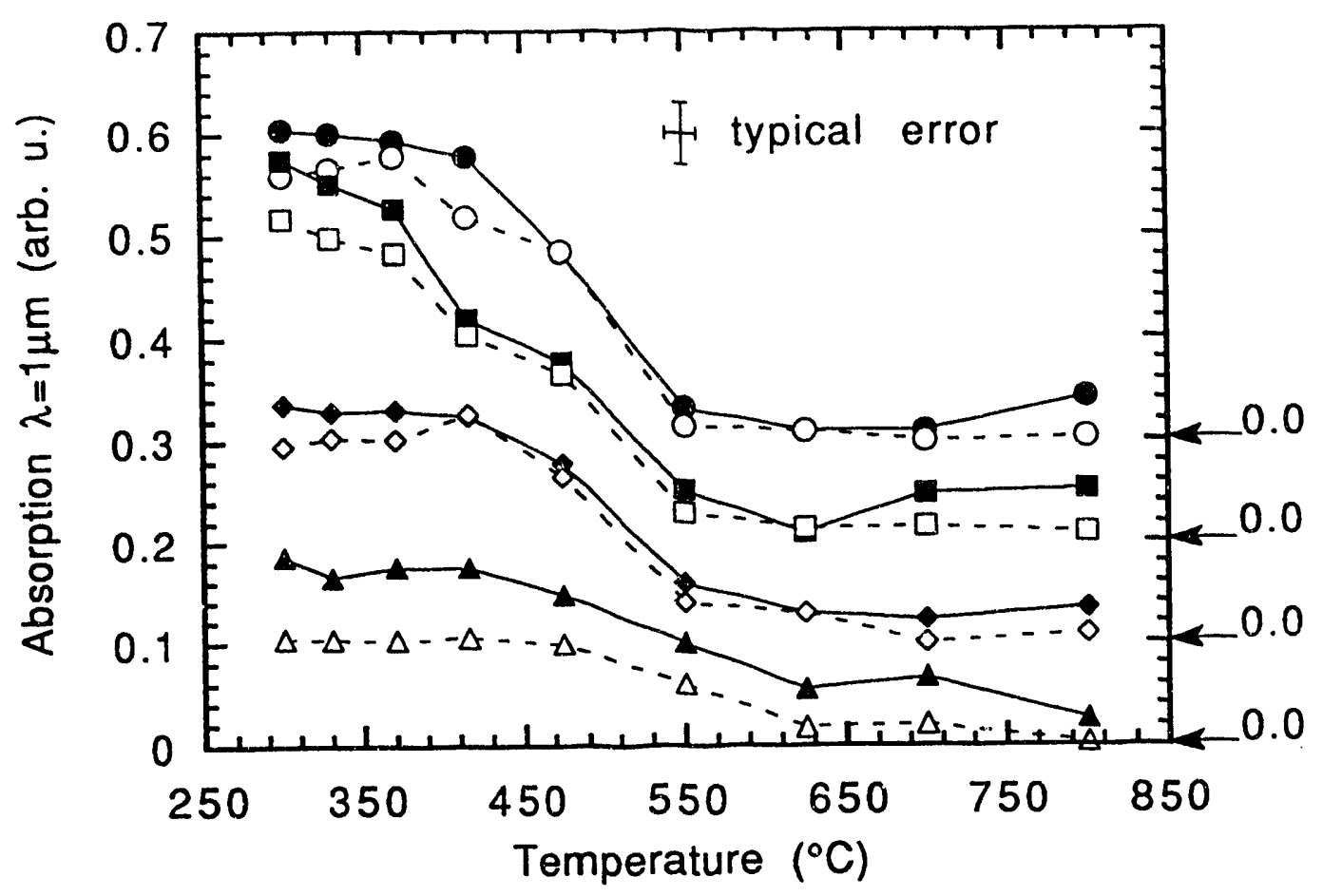

FIG. 3.1 IR absorption at a wavelength of $1 \mu \mathrm{m}$ and temperature of $10 \mathrm{~K}$ of isochronally annealed LTMBE GaAs:Be. The plots are offset for easier viewing. Absorption is proportional to neutral AsGa-related defect concentration. Filled in symbols connected with solid lines are for the total defect absorption before illumination. Open symbols connected with broken lines are the ratios of absorption before/after 30 minute white light illumination, representing the photoquenchable portion of the defect absorption $\left(\bullet-[B e]=10^{16} \mathrm{~cm}^{-3}\right.$, $\left.[\mathrm{Be}]=10^{17} \mathrm{~cm}^{-3},-[\mathrm{Be}]=10^{18} \mathrm{~cm}^{-3}, \Delta-[\mathrm{Be}]=10^{19} \mathrm{~cm}^{-3}\right)$.

annealing temperatures below $430^{\circ} \mathrm{C}$. This is more obvious in the Si-doped layers (see Fig. 3.2) which contain more NPQ defects and already begin to anneal at $330^{\circ} \mathrm{C}$, the lowest temperature heat treatment.

One can apply the simple rule of thumb (eq. 2.1) to convert the temperature of the annealing stage into an activation energy. The annealing of the $P Q$ defects at $450^{\circ} \mathrm{C}$ corresponds to an activation energy of approximately $1.8 \mathrm{eV}$. On the other hand, the less stable $\mathrm{NPQ}$ defects already begin to anneal away at $330^{\circ} \mathrm{C}$. Thus an upper 


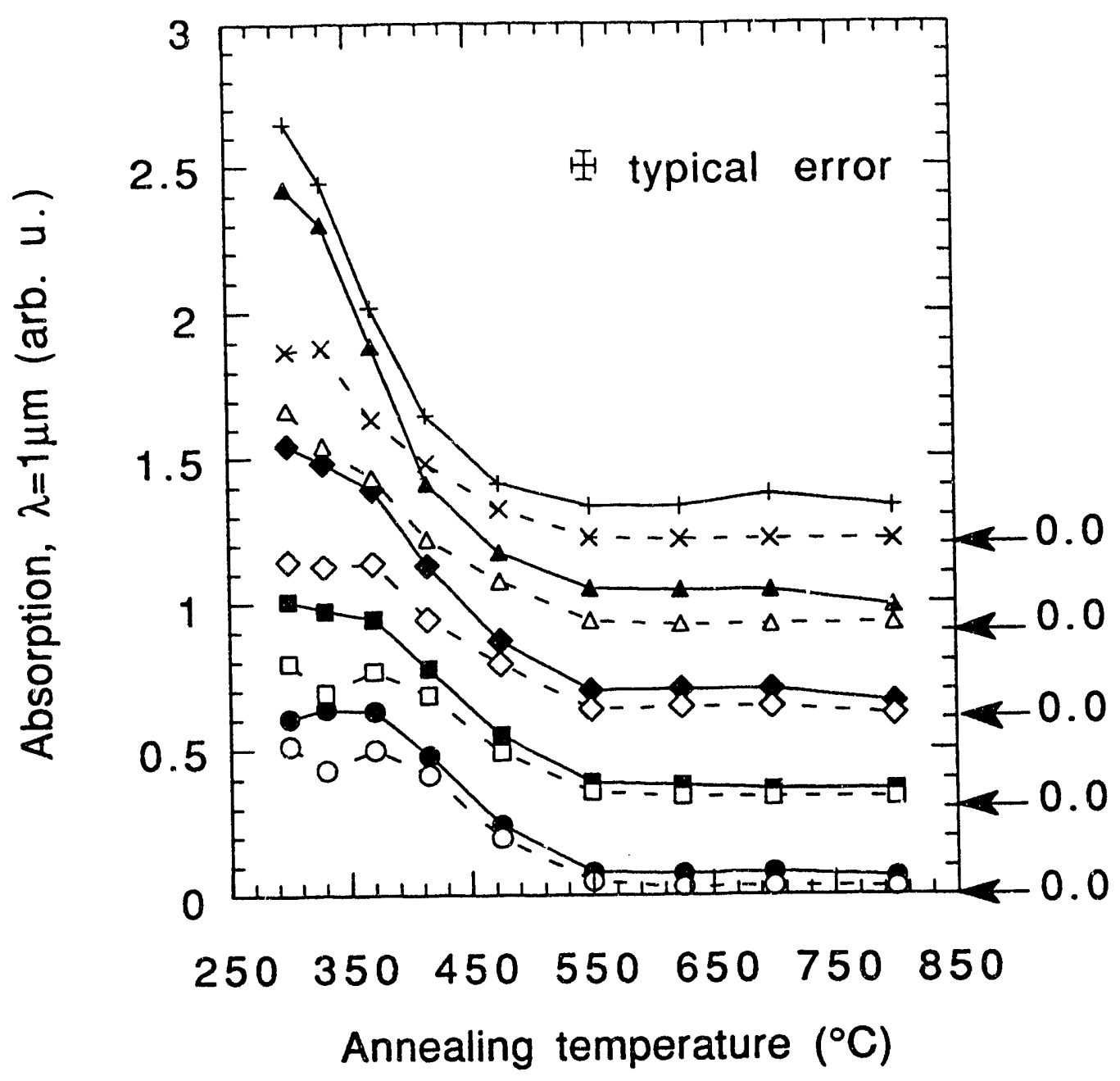

FIG. 3.2 IR absorption at a wavelength of $1 \mu \mathrm{m}$ and temperature of $10 \mathrm{~K}$ of isochronally annealed LTMBE GaAs:Si. The plots are offset for easier viewing. Zero points are indicated on the right-hand axis. Absorption is proportional to neutral AsGa-related defect concentration. Filled in symbols connected with solid lines are for the total defect absorption before illumination. Open symbols connected with broken lines are the ratio of absorption before/after 30 minute white light illumination, representing the photoquenchable portion of the defect absorption (- undoped, - - $[\mathrm{Si}]=10^{16} \mathrm{~cm}^{-3},-[\mathrm{Si}]=10^{17} \mathrm{~cm}^{-3}$, $\left.\Delta-[\mathrm{Si}]=10^{18} \mathrm{~cm}^{-3},+-[\mathrm{Si}]=10^{19} \mathrm{~cm}^{-3}\right)$.

limit can be placed on the activation energy for the annealing of the NPQ defects. It must be less than approximately $1.5 \mathrm{eV}$.

At this point a definitive explanation can not be given for the lower stability of NPQ versus PQ AsGa-related defects. The exact 
atomic structure of both defects is still unknown. Nevertheless, the resu!!ts in cirapter 2 which suggest that the lack of metastability of some AsGa-related defects is due to the presence of neighboring defects, appears to be consistent with the lower thermal stability of NPQ derects versus their PQ counterparts. It seems reasonable that two interacting defects in close proximity could combine more easily than isolated defects to form a complex. The more isolated $P Q$ AsGa-related defects must migrate further to find a sink or defect with which to complex and anneal away.

Without becoming involved in a lengthy discussion at this point, it is interesting to compare the lower stability of AsGarelated defects in LTMBE. GaAs to EL2 in bulk grown crystals which is stable up to $1000{ }^{\circ} \mathrm{C}$. Because of the difference in annealing characteristics, some researchers have proposed that the AsGarelated defects in LTMBE GaAs are different from EL2. I believe however that the AsGa-related defects are very similar if not identical to EL2. The lower annealing temperature for the defects is not ciue to an inherently less stable defect but rather the enhanced diffusivity of atoms in LTMBE GaAs. The lower temperature annealing of AsGa-related defects is die to defect enhanced diffusion of AsGa to precipitates. In this case, it is proposed that the presence of excess $V_{G a}$ assists the diffusion of $A s G a$. The implications of $V_{G a}$ aided diffusion of $A s G a$ will be considered in the discussion following the isothermal annealing results. 


\subsubsection{Electrical characterization}

Dependence of hopping conductivity on annealing temperature

The dependence of the electrical properties on the isochronal annealing temperature was investigated by high impedance Hall effect and resistivity measurements. The variable temperature resistance of the $[\mathrm{Be}]=10^{17} \mathrm{~cm}^{-3}$ doped sample is shown in Fig. 3.4 for annealing temperatures up to $550^{\circ} \mathrm{C}$. Above the annealing temperature of $550^{\circ} \mathrm{C}$ the resistivity of the LTMBE layer becomes so great that electrical measurements are dominated by parallel conductivity in the LEC substrate.

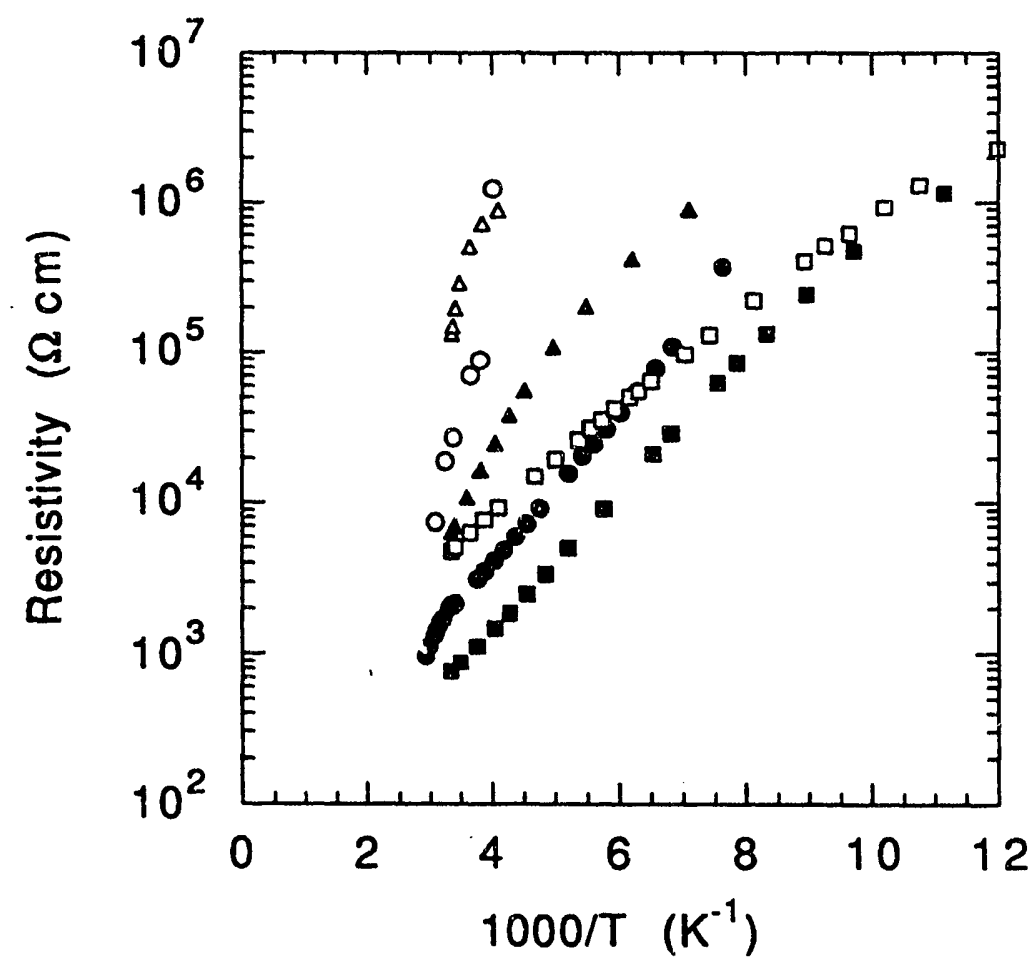

FIG. 3.3 Temperature dependent resistivity of isochronally annealed undoped LTMBE GaAs. The annealing time was 20 minutes. The initial steep slope above room temperature is due to the parallel conductivity of the substrate. The shallower low temperature slope is thermally activated hopping conduction in the layer. Hopping conductivity is reduced in annealed samples. Annealing temperatures were: - as-grown, $-330^{\circ} \mathrm{C},-370^{\circ} \mathrm{C}, \mathrm{O}-$ $415^{\circ} \mathrm{C}, \square-470^{\circ} \mathrm{C}, \Delta-550^{\circ} \mathrm{C}$. 
Similar to the as-grown Be-doped sample, the resistivity of the annealed samples shows two regions of thermally activated behavior which can be described by eq. 2.6 in section 2.2.3. For increasing annealing temperatures, the low temperature region described by the parameters $\rho_{3}$ and $\varepsilon_{3}$ moves towards higher resistivity indicating a reduction of hopping conductivity. The resistivity of the $1017 \mathrm{~cm}^{-3}$ sample at $250 \mathrm{~K}$ increases nearly 3 orders of magnitude after annealing at $550^{\circ} \mathrm{C}$. The defect concentration, however, decreases by only a factor of 8 for the same annealing temperature (see Fig. 3.1). This strong dependence of the resistivity on the defect concentration is further evidence for a hopping conduction mechanism. The hopping probability to an empty site is proportional to the electron wavefunction overlap which drops off exponentially with distance. As AsGa defects anneal away the concentration of hopping centers decreases and the mean separation between centers increases. Since the electron wavefunction overlap depends exponentially on the separation between the defects, the reduction in defect concentration leads to an exponential increase in resistivity. This process is analogous to an electron tunneling through a barrier and the width of the barrier is increased.

A model by Shklovskii and Efros (1984a) has been developed which treats hopping conduction over shallow impurities in lightly compensated semiconductors, $K=N_{A} / N_{D}<10^{-3}$. The model has had good success predicting the behavior of hopping conduction over shallow levels and over deep levels for the case of InP:Mn (Kuznetsov, et al. 1984). Attempts to analyze the temperature 


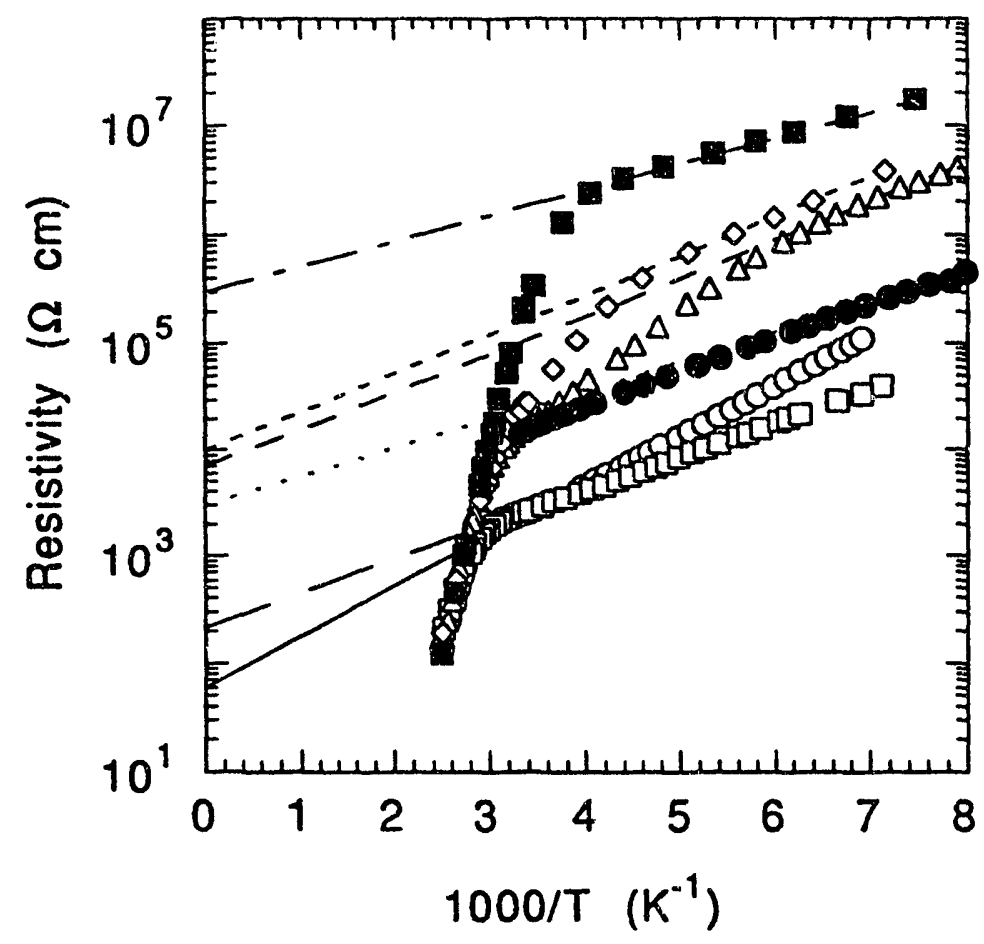

FIG. 3.4 Temperature dependent resistivity of isochronally annealed LTMBE GaAs:Be. The annealing time was 20 minutes. The initial steep slope above room temperature is due to the parallel conductivity of the substrate. The shallower low temperature slope is due to thermally activated hopping conduction in the layer. Hopping conductivity is reduced in annealed samples. Annealing temperatures were: 0 - as-grown, $\square-330^{\circ} \mathrm{C}, \Delta-370^{\circ} \mathrm{C}, \nabla-415^{\circ} \mathrm{C}$, $-470^{\circ} \mathrm{C},-550^{\circ} \mathrm{C}$. Straight lines are expone ual fits to the resistivity in the nearest neighbor hopping regime. Values of $p_{3}$ and $\varepsilon_{3}$ determined from this fit are summarized in Table 3.1.

dependent resistivity data of LTMBE GaAs in terms of this single level hopping model have only had limited success. The trends, however, are accurate and the analysis serves to illustrate that the annealing process involves more than one electrically active defect.

For hopping conduction between neighboring states, the resistivity obeys the relation, $\rho=\rho_{3} \exp \left\{\varepsilon_{3} / k T\right\}$. Shklovskii (1984b) uses percolation theory to derive expressions for the activation energy, $\varepsilon_{3}$, and the infinite temperature resistivity, $\rho_{3}$. The 
theoretical activation energy depends only on the concentration of the percolation level, the dielectric constant of the material, and the compensation ratio,

$$
\varepsilon_{3}=\frac{e^{2} N^{-1 / 3}}{\epsilon}\left(1-0.29 \mathrm{~K}^{1 / 4}\right)
$$

where $e$ is the electric charge, $N$ is the donor concentration, $\in$ is the dielectric constant and $\mathrm{K}$ is the compensation ratio. For the samples with a measured concentration of $E L 2,3 \times 10^{19} \mathrm{~cm}^{-3}$, the calculated activation energy is $30 \mathrm{meV}$. This is a factor of 2 to 3 too low compared to values of $\varepsilon_{3}$ in Table 3.1.

The solid lines in Fig. 3.3 are the fit to $\rho_{3} \exp \left\{\varepsilon_{3} / k T\right\}$ in the low temperature limit. Table 3.1. gives the values of $\rho_{3}$ and $\varepsilon_{3}$ along with the EL2 like defect concentration.

For the intercept term, $\rho_{3}$, percolation theory gives the relation

$$
\rho_{3}=\rho_{3}^{0} \exp \left[\alpha /\left(\mathrm{N}^{1 / 3} a\right)\right]
$$

where $\alpha=1.73 \pm 0.03$ (Shklovskii and Efros 1984b). According to equation 3.2, plotting the $\ln \left(P_{3}\right)$ versus $N^{-1 / 3}$ should yield a straight line of slope equal to $\alpha / a$, where $a$ is the state radius of the wavefunction decaying at large distances according to $\Psi(r) \propto$ $\exp (-r / a)$. Figure 3.5. plots $P_{3}$ versus $N^{-1 / 3}$ of the annealed samples taken from Table 3.1. The points do not lie on a straight line, but the trend is as predicted, the resistivity increases as the mean inter- 
impurity distance, $\mathrm{N}^{-1 / 3}$, increases. The dashed line corresponds to a localization radius, $a=8 \AA$. This small value determined for $a$ indicates that defects comprising the hopping level are much more localized than shallow hydrogenic levels. The value of $a$ is on the order of a bond length in GaAs, $2.45 \AA$ rather than the hydrogenic level Bohr radius, $\sim 160 \AA$. The wave function overlap is determined experimentally by electrons hopping between defects separated by distances corresponding to $N^{-1 / 3}=30 \AA$. Extrapolating the wave function overlap at great distances back to the central cell of the defect does not give a physically accurate picture of the defect structure. Rather, $a=8 \AA$ should be considered a parameter indicating that the EL2-like defect responsible for hopping conduction is highly localized.

Table 3.1 Values of $\varepsilon_{3}$ and $\rho_{3}$ taken from a low temperature fit of the resistivity data in Fig. 3.3 for LT MBE GaAs:Be $1017 \mathrm{~cm}^{-3}$ after annealing. EL2 like defect concentration is measured by IR absorption at $1 \mu \mathrm{m}$ as seen in Figure 3.1.

\begin{tabular}{cccr}
\hline $\begin{array}{c}\text { Anneal } \\
\begin{array}{c}\text { Temperature } \\
\left({ }^{\circ} \mathrm{C}\right)\end{array}\end{array}$ & $\begin{array}{c}\text { EL2 Like } \\
\text { Defect Conc. } \\
\left(\times 10^{19} \mathrm{~cm}^{-3}\right)\end{array}$ & $\begin{array}{c}\varepsilon_{3} \\
(\mathrm{meV})\end{array}$ & $\begin{array}{c}\rho_{3} \\
(\Omega \mathrm{cm})\end{array}$ \\
\hline As grown & $2.9 \pm 0.2$ & 93 & 60 \\
330 & $2.7 \pm 0.2$ & 63 & 215 \\
370 & $2.5 \pm 0.2$ & 65 & 7,000 \\
415 & $1.7 \pm 0.2$ & 71 & 10,000 \\
475 & $1.4 \pm 0.2$ & 61 & 3000 \\
550 & $0.4 \pm 0.2$ & 46 & 300,000 \\
\hline
\end{tabular}




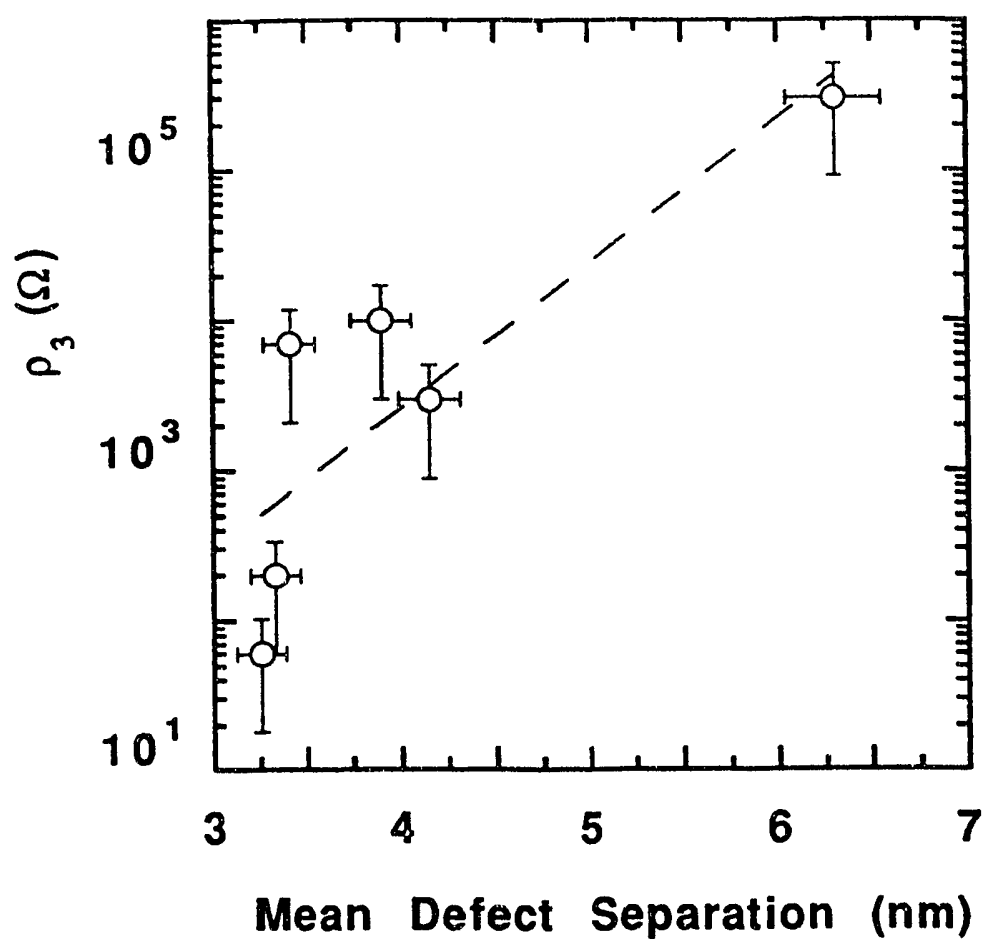

FIG. 3.5 Plot of $P_{3}$ versus the mean defect separation, $N^{-1 / 3}$. The dashed line corresponds to a localization radius of $8 \AA$.

If annealing had only reduced the concentration of levels responsible for hopping, in this case the $A s_{G_{a}}$-related level, then the resistivity curves should have fanned out smoothly. The slope, $\varepsilon_{3}$, and spacing, $\rho_{3}$, between successive curves should have increased proportional to $\mathrm{N}^{-1 / 3}$. Instead, it should be noted that the temperature dependent resistivity curves in Fig. 3.3 do not increase smoothly with annealing temperature. After the first anneal at $330^{\circ} \mathrm{C}, \varepsilon_{3}$ decreases initially. After the second anneal at $370^{\circ} \mathrm{C}$ the resistivity and slope increase dramatically but after the next anneal at $420^{\circ} \mathrm{C}$ the resistivity drops back down again. This crossing of the resistivity curves with annealing is reproducible in these samples and is not due to errors in annealing temperatures. Therefore, I 


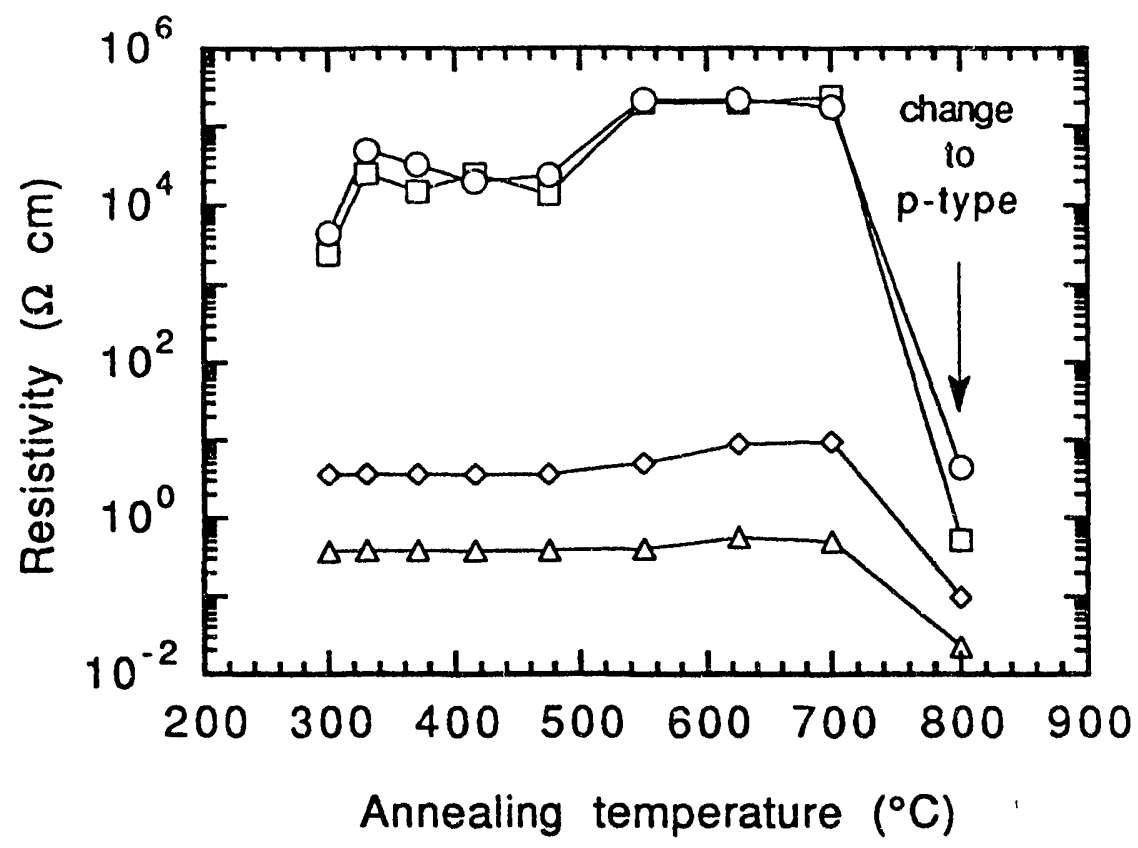

FIG. 3.6 Room temperature resistivity of isochronally annealed samples. Annealing time was 20 minutes. Resistivity increases initially as defects anneal away. The $2 \times 10^{5} \Omega \mathrm{cm}$ plateau is due to the parallel conductivity of substrate. Free isoles are activated to the valence band after annealing at $800^{\circ} \mathrm{C}$. $(0-$ $[\mathrm{Be}]=10^{16} \mathrm{~cm}^{-3}, \square-[\mathrm{Be}]=10^{17} \mathrm{~cm}^{-3}, \diamond-[\mathrm{Be}]=10^{18} \mathrm{~cm}^{-3}, \Delta-[\mathrm{Be}]=$ $10^{19} \mathrm{~cm}^{-3}$ ). Estimated errors are $\pm 10^{\circ} \mathrm{C}$ in temperature and $20 \%$ of the resistivity value.

believe other electrically active defects are annealing. away and changing the occupation of the hopping level. Changes in the compensation ratio, $\mathrm{K}$, will affect the activation energy $\varepsilon_{3}$ as described by eq. 3.1 . It is possible that annealing at $420^{\circ} \mathrm{C}$, the temperature at which the resistivity curves crossover, is an annealing stage of an acceptor-like native defect.

Figure 3.6 shows the room temperature resistivity versus isochronal annealing temperature for the 1016 and $1017 \mathrm{~cm}^{-3} \mathrm{Be}$ doped samples. The resistivity increases with annealing temperature until a maximum of $2 \times 10^{5} \Omega \mathrm{cm}$ is reached at $550^{\circ} \mathrm{C}$. We 
attribute the increase in resistivity to a reduction in the concentration of hopping centers as just described. The plateau of maximum resistivity is the point at which the parallel resistivity of the SI substrate is again less than the layer and dominates the measurement.

\section{Electrical activation of $\mathrm{Be}$}

After annealing at $800^{\circ} \mathrm{C}$ all the layers become strongly $\mathrm{p}$ type, including the shunted, highly doped layers. Table 3.2 summarized the Hall effect measurements on the $800^{\circ} \mathrm{C}$ annealed layers. The free hole concentration is roughly $60-80 \%$ of the intended Be doping. This is within the accuracy of both the doping during growth, $20 \%$, and the accuracy of the Hall measurement, $20 \%$. The fraction of Be activated appears to be constant from sample to sample. The anomalously high activation of the $1016 \mathrm{~cm}^{-3}$ doped sample is probably due to the fact that the doping is extremely hard to control at such a low concentration. Normally the crystal growers do not dope at such a low concentration, so their calibration could be in error.

A striking feature of the results shown in Fig. 3.6 is that even though the AsGa-related donors rapidly anneal off at $550^{\circ} \mathrm{C}$ we do not observe any electrical activity of $\mathrm{Be}$ acceptors below $700^{\circ} \mathrm{C}$. Since our Raman scattering experiments show that most of the $\mathrm{Be}$ atoms are substitutional we have to conclude that a large concentration of AsGa-related donor defects is stable up to $700^{\circ} \mathrm{C}$.

A possible explanation for the enhanced stability of the donors is that they are complexed with substitutional $\mathrm{Be}$ acceptors, 
TABLE 3.2 Summary of Be activation determined by room temperature Hall effect measurements. Samples were annealed at $800^{\circ} \mathrm{C}$ for $20 \mathrm{~min}$. in a sealed ampul with $\sim 2 \mathrm{~atm}$. As overpressure.

\begin{tabular}{cccc}
\hline $\begin{array}{c}\text { Intended } \\
\text { Be doping } \\
\left(\mathrm{cm}^{-3}\right)\end{array}$ & $\begin{array}{c}\rho \\
\left(\mathrm{cm}^{-3}\right)\end{array}$ & $\begin{array}{c}\rho \\
(\Omega \mathrm{cm})\end{array}$ & $\begin{array}{c}\mu \\
\left(\mathrm{cm}^{2} / \mathrm{Vs}\right)\end{array}$ \\
\hline $10^{16}$ & $4.2 \times 10^{16}$ & 4.42 & 33 \\
$10^{17}$ & $7.9 \times 10^{16}$ & 0.53 & 150 \\
$10^{18}$ & $6.4 \times 10^{17}$ & 0.098 & 100 \\
$10^{19}$ & $5.7 \times 10^{18}$ & 0.023 & 48 \\
\hline \hline
\end{tabular}

forming $\mathrm{As}_{\mathrm{Ga}}^{+}+\mathrm{Be}_{\mathrm{Ga}}^{-}$donor-acceptor pairs. lonized $\mathrm{As}_{\mathrm{Ga}}^{+}$defects tied up in the pairs would not contribute to IR absorption and therefore could not be detected in absorption measurements. The activation of $\mathrm{Be}$ acceptors at temperatures exceeding $700^{\circ} \mathrm{C}$ is associated with a break up of $\mathrm{As}_{\mathrm{Ga}}^{+}+\mathrm{Be}_{\mathrm{Ga}}^{-}$pairs and subsequent diffusion of AsGa to As precipitates. A simple calculation can give the electrostatic binding energy of the $\mathrm{As}_{\mathrm{Ga}_{a}}^{+}+\mathrm{Be}_{\mathrm{Ga}}^{-}$pair located on neighboring sites of the $\mathrm{Ga}$ sublattice. The electrostatic energy for two point charges is $E=e^{2 / \epsilon} r$, where $e$ is the charge of an electron, $\epsilon=13$ is the macroscopic d.c. dielectric constant and $r=a_{d} \sqrt{2}=4 \AA$ is the Ga-Ga separation which yields an electrostatic attraction of about $0.3 \mathrm{eV}$. This estimate gives a lower limit to the actual binding energy since the local dielectric constant for the closely spaced $\mathrm{As}_{\mathrm{G}_{a}}^{+}+\mathrm{Be}_{\mathrm{Ga}}^{-}$pair is less than the macroscopic dielectric constant. This is due to the presence of only a few atoms between the pair which would not be able to screen the field as effectively as a macroscopic field applied across the entire crystal. 
In any case, the actual binding energy of the $\mathrm{As}_{\mathrm{Ga}}^{+}+\mathrm{Be}_{\mathrm{Ga}}^{-}$pair would have to be calculated from the change in Gibbs free energy as the two atoms are brought together from an infinite separation. Such a determination would take into account the relaxation of the lattice around the defect (This would serve to increase the binding energy of the pair as well since the smaller $\mathrm{Be}$ atom and larger $\mathrm{As}$ atom would have opposite strain fields.) and the change in entropy (which is unknown) of the system by bringing the atoms together.

Using our simple calculation and applying the energytemperature rule of thumb, the annealing temperature necessary to break up electrostatically attracted $\mathrm{As}_{\mathrm{G}_{a}}^{+}+\mathrm{Be}_{\mathrm{Ga}}^{-}$pairs would have to be at least $150^{\circ} \mathrm{C}$ higher and probably more due to our low estimate of the binding energy. This is consistent with the observed activation of $\mathrm{Be}$ for annealing temperatures above $700^{\circ} \mathrm{C}$.

Electrical activation of $\mathrm{Si}$

Figure 3.7 shows the dependence of the variable temperature resistivity on annealing temperature of the sample doped with [Si]=1019 $\mathrm{cm}^{-3}$. The hopping conductivity of this sample is representative of the other $\mathrm{Si}$ doped samples. The x-axis of Fig. 3.7 is scaled as $\mathrm{T}^{-1 / 4}$ to emphasize the region of variable range hopping conductivity as a straight line. Although the Be-doped samples show a nearest neighbor hopping mechanism (Fig. 3.3) as opposed to variable range hopping in the Si-doped samples, both the $\mathrm{Si}$ - and $\mathrm{Be}$ doped samples exhibit a similar increase in resistivity with 


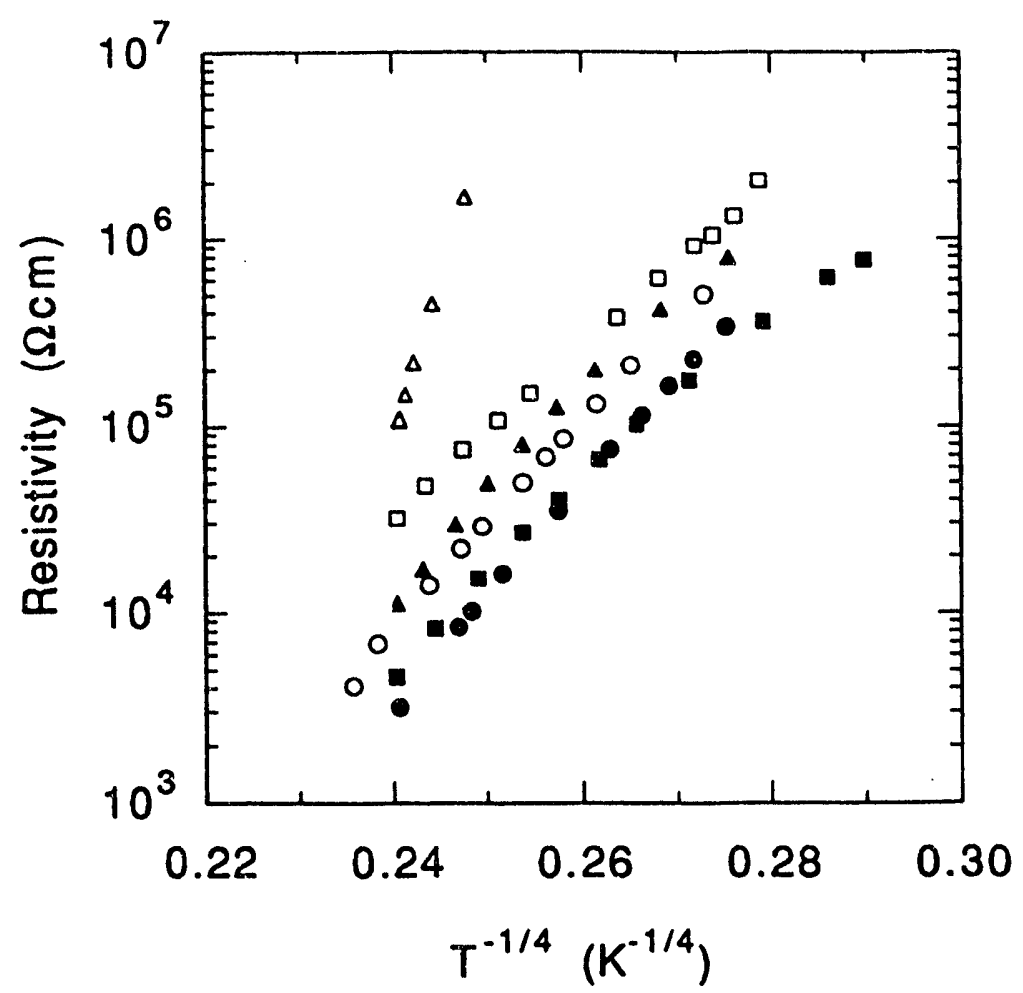

FIG. 3.7 Temperature dependent resistivity of isochronally annealed LTMBE GaAs:Si $10^{19} \mathrm{~cm}^{-3}$ plotted as a function of $\mathrm{T}^{-1 / 4}$ to illustrate variable range hopping. The annealing time was 20 minutes. Hopping conductivity is reduced in annealed samples. Annealing temperatures were: - as-grown, $\square-330^{\circ} \mathrm{C}, \triangle-370^{\circ} \mathrm{C}, 0-415^{\circ} \mathrm{C}, \square-470^{\circ} \mathrm{C}, \Delta-550^{\circ} \mathrm{C}$.

annealing temperature. Again, the hopping conductivity is reduced as the AsGa-related defects, which, are responsible for the hopping, anneal away. At $800^{\circ} \mathrm{C}$ the $\mathrm{Si}$ is only partially activated and the samples become more strongly n-type. As a point of comparison, $800-850^{\circ} \mathrm{C}$ is the annealing temperature range required to activate ion implanted dopant species. Thus it appears some fundamental process occurs in GaAs at these temperatures. In the case of the $\mathrm{Be}$, which we know to already occupy substitutional sites, the activation process is related to the annealing of defects or defectimpurity complexes. In the case of the $\mathrm{Si}$ it is not clear whether the 


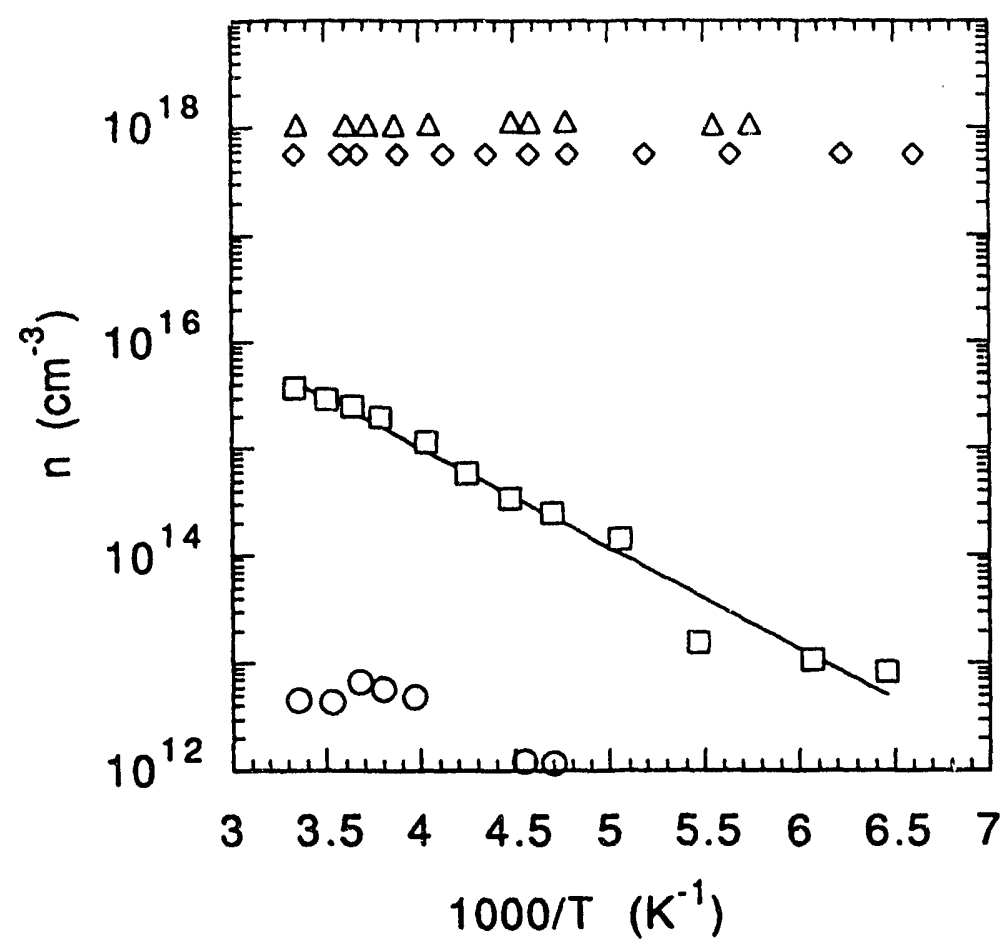

FIG. 3.8 Variable temperature Hall effect measurements of LTMBE GaAs:Si annealed at $800^{\circ} \mathrm{C}$ for 20 minutes. The Si is only partially activated. Symbols correspond to a Si concentration of: $0-10^{16} \mathrm{~cm}^{-3}, \square-10^{17} \mathrm{~cm}^{-3}$, $\Delta-10^{18} \mathrm{~cm}^{-3}, 0-10^{19} \mathrm{~cm}^{-3}$. The straight line is an exponential fit with an activation energy of $185 \mathrm{meV}$.

$800^{\circ} \mathrm{C}$ annealing stage is a similar defect annealing stage or if it corresponds to moving the $\mathrm{Si}$ to a substitutional site.

Figure 3.8 shows the variable temperature free carrier concentration determined by Hall effect measurements of the four $\mathrm{Si}$ doped layers annealed at $800^{\circ} \mathrm{C}$ for 20 minutes. The two more highly doped samples, $10^{18}$ and $10^{19} \mathrm{~cm}^{-3}$, exhibit free carrier conductivity characteristic of a degenerately doped shallow donor, i.e., a temperature independent free electron concentration. In the sample doped $1017 \mathrm{~cm}^{-3}$ the carriers freeze-out with an activation energy of approximately $186 \mathrm{meV}$. This corresponds to the Fermi energy being pinned to a level at $160 \mathrm{meV}$ below the conduction band 
edge when corrected for the $T^{3 / 2}$ dependence of the density of states. The sample doped with [Si] $=10^{16} \mathrm{~cm}^{-3}$ still exhibits hopping conductivity afte $i$ the $800^{\circ} \mathrm{C}$ anneal. The results of room temperature Hall effect and resistivity measurements on the $800^{\circ} \mathrm{C}$ annealed Si doped samples are summarized in Table 3.2.

The mobility of the samples annealed at $800^{\circ} \mathrm{C}$ is greater than for the the as-grown samples but it is not as high as in bulk material. The dominant free carrier scattering mechanism at room temperature is ionized impurity scattering. Walukiewicz et, al. (1979) has calculated the mobility as a function of free carrier concentration and compensation. Using the mobility calculations (Walukiewicz, et al. 1979, see Table II) the compensation ratio in our samples can be estimated as well as the total donor concentration. The results of this determination are summarized in Table 3.3. We see that the compensation of the donors is high, more than $50 \%$. This indicates that the total donor concentration is on the order of the intended $\mathrm{Si}$ doping to within the accuracy of the calculation. What this suggests is that residual acceptor defects compensate the shallow donors. The concentration of acceptors is determined to be greater than $1017 \mathrm{~cm}^{-3}$ since the [Si] $=10^{18} \mathrm{~cm}^{-3}$ but not the $[\mathrm{Si}]=10^{17} \mathrm{~cm}^{-3}$ shows the Fermi level to be near the band edge. Also the concentration of compensating accepiors appears tied to the $\mathrm{Si}$ concentration. The $[\mathrm{Si}]=10^{19} \mathrm{~cm}^{-3}$ doped sample contains more residual acceptors than the $[S i]=10^{18} \mathrm{~cm}^{-3}$ doped sample. 
TABLE 3.3 Summary of the room temperature electrical properties of $800^{\circ} \mathrm{C}$ annealed LTMBE GaAs:Si. $\kappa$ is the compensation ratio determined from the mobility as outlined by Walukiewicz et. al. (1979). $N_{d}$ is the calculated total donor concentration determined from $n$ and $\mu$. The $10^{16} \mathrm{~cm}^{-3}$ Si-doped layer exhibits hopping conductivity. Hence $n$ does not correspond to a free carrier concentration. The $10^{17} \mathrm{~cm}^{-3}$ Si-doped layer freezes out from room temperature hence $E_{\mathfrak{f}}$ is pinned to a level below the Si level.

\begin{tabular}{cccc|cc}
\hline $\begin{array}{c}\text { Si doping } \\
\left(\mathrm{cm}^{-3}\right)\end{array}$ & $\begin{array}{c}n \\
\left(\mathrm{~cm}^{-3}\right)\end{array}$ & $\begin{array}{c}\rho \\
(\Omega \mathrm{cm})\end{array}$ & $\begin{array}{c}\mu \\
\left(\mathrm{cm}^{2} / \mathrm{Vs}\right)\end{array}$ & $\kappa$ & $\begin{array}{c}\left.N_{\mathrm{d}}=\mathrm{n} / 1-\mathrm{k}\right) \\
(\mathrm{cm}-3)\end{array}$ \\
\hline $10^{16}$ & $4.6 \times 10^{12}$ & $1.3 \times 10^{4}$ & 102 & $\ldots \ldots \ldots$ & $\ldots \ldots \ldots$ \\
$10^{17}$ & $3.8 \times 10^{15}$ & 1.6 & 1050 & $\ldots \ldots$. & $\ldots \ldots \ldots$ \\
1018 & $5.6 \times 10^{17}$ & $6.0 \times 10-3$ & 1830 & 0.60 & $1.4 \times 10^{18}$ \\
1019 & $1.1 \times 10^{18}$ & $6.6 \times 10-3$ & 890 & 0.77 & $4.8 \times 10^{18}$ \\
\hline \hline
\end{tabular}

The fact that the $[S i]=10^{19} \mathrm{~cm}^{-3}$ shows only $10 \%$ activation after annealing at $800^{\circ} \mathrm{C}$ is not unexpected. It has been well documented from bulk doping, diffusion and ion implantation experiments that a saturation limit of approximately $3 \times 10^{18} \mathrm{~cm}^{-3}$ exists for the free electron concentiation. A mechanism has been proposed to explain this free carrier saturation phenomenon. It involves the transformation of amphoteric defects between donoror acceptor-like configurations depending on the position of the Fermi level relative to the defect stabilization level. In GaAs the defect stabilization level is positioned closer to the valence band than conduction band edge favoring the formation of acceptor-like native defects when the free electron concentration becomes large.

Overcoming this saturation limit for n-type doping would be a technological milestone for GaAs devices. The current saturation concentration is less than ideal for heavily doped regions of GaAs devices such as in the base of bipolar transistors and near contact regions. The doping restriction increases the resistivity and hence 
RC time constants of devices. Unfortunately, the incorporation of donor dopants during low temperature growth and subsequent annealing does not appear to be a successful means of exceeding this saturation limit at this point.

\subsection{Isothermal annealing experiments}

Once isochronal anneals have been completed, the temperature at which specific annealing stages occur is known. One can then perform isothermal anneals just below the transition temperature to study the kinetics of the annealing process. These studies were performed to better understand the kinetics of the AsGa annealing stage at around $500^{\circ} \mathrm{C}$. The annealing of AsGa-related defects will also be referred to as a recovery process. The reason being that the large excess concentration of defects are recovering to equilibrium concentrations during annealing of the layers. By analyzing the temperature dependence of the rate at which defect concentrations recover, much insight into the energetics and kinetics of defect transformations can be gained. The functional dependence of the defect concentration on time yields information on whether the recovery process is first order, second order or more complicated. An initial period of no detectable change, also known as an incubation period, might signal the need to form precipitates before a precipitation process starts. The dependence of the recovery rate on temperature can yield an activation enthalpy for the process. The concentration dependence of the annealing rate yields further information about possible recovery mechanisms. 


\subsubsection{Isothermal annealing conditions}

Prior to annealing, the backside of the substrate was polished for optical measurements. The Be-doped samples were isothermally annealed at 420,450 and $490^{\circ} \mathrm{C}$ in flowing $\mathrm{N}_{2}$. The Si-doped samples were annealed at slightly lower temperatures, 400,440 and $480^{\circ} \mathrm{C}$, The isochronal annealing of the Si-doped layers indicated that the annealing stage was at slightly lower temperatures. Annealing of the samples was interrupted at specific time intervals. The samples were removed to make room temperature IR absorption measurements in the CARY spectrometer and then placed back in the furnace. Before each absorption measurement samples were rinsed in $30 \% \mathrm{HF}$ to remove any arsenic deposits on the surface which might scatter light.

\subsubsection{Isothermal Annealing Results}

To determine the dependence of the AsGa-related defect concentration on annealing time, the IR absorption at $1 \mu \mathrm{m}$ was measured relative to the background absorption at $1.7 \mu \mathrm{m}$ while the sample was at room temperature. The $1 \mu \mathrm{m}$ absorption is due to the neutral charge state of the AsGa-related defect. Since we have shown that the AsGa-related defect is lightly compensated, the change in absorption represents the change in the total concentration of AsGa-related defects. Unfortunately, room temperature IR absorption measurements cannot distinguish between $P Q$ and NPQ AsGa-related defects. Low temperature IR absorption measurements could have separated the annealing of each type of defect, but the requirement to cool to liquid helium temperatures would have been prohibitively slow and expensive. 


\section{Undoped lavers}

For the undoped layers, the normalized concentration of neutral AsGa-related defects is plotted versus the annealing time in Fig's. 3.9 (a) and (b). Figure 3.9 (a) is a semi-log plot of the recovery transient at the three isothermal annealing temperatures, 400, 450 $490{ }^{\circ} \mathrm{C}$. The solid and dashed lines are fits to the data described in the following discussion section. The semi-log plot shows that the annealing transient is not a single exponential which would have been represented as a straight line extending from 1 to $-\infty$. Instead the annealing proceeds slower with time. Especially at the lowest annealing temperature, it appears that some fraction of the defects never anneal away. The saturation of the annealing process is indicated by the offset in the curve for long annealing times. It is intuitively apparent why the defect annealing rate slows. We have proposed that the annealing process is the recovery of a supersaturated concentration of AsGa-related defects. The recovery to equilibrium concentrations occurs via the $V_{G a}$ enhanced diffusion of AsGa-related defects to arsenic precipitates. However, in addition, the concentration of $V_{G a}$ which enable the diffusion is also supersaturated and must also recover. As the $V_{G a}$ concentration decreases, the diffusivity of the AsGa also decreases, resulting in progressively slower annealing. Eventually the concentration of $\mathrm{V}_{\mathrm{Ga}}$ decreases sufficiently that the AsGa diffusion comes to an effective halt. This is why the recovery does not go to completion and we see a non-zero residual concentration of AsGa-related defects after low temperature annealing. 

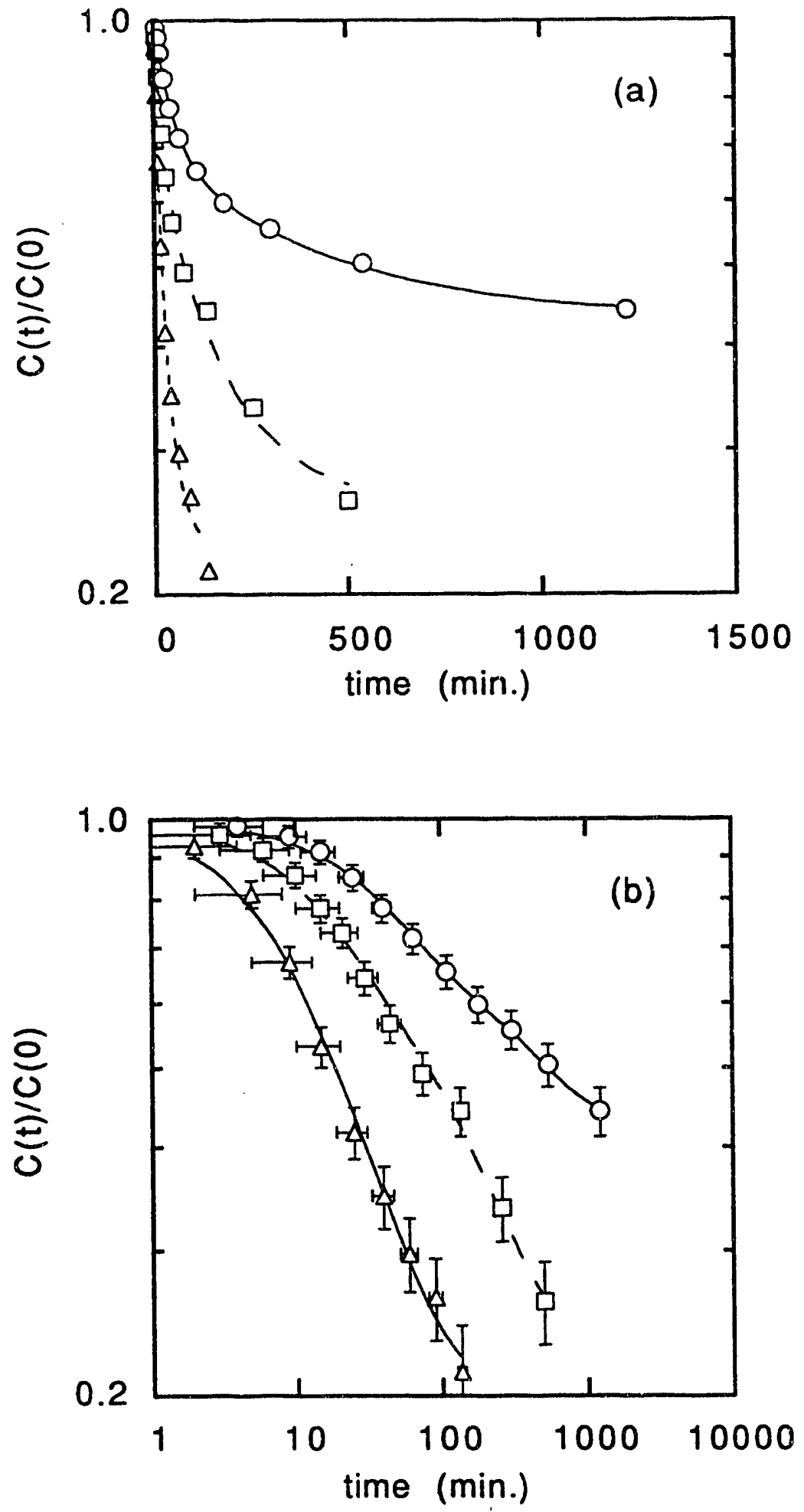

FIG. 3.9 Isothermal annealing transients for the undoped LTMBE GaAs layer. (a) is a semi-log plot of the normalized concentration of $A_{s_{G a}}$-related defect versus isothermal annealing time. (b) is a log-log plot. Annealing temperatures are: $0420^{\circ} \mathrm{C}, \square 450^{\circ} \mathrm{C}, \Delta 490^{\circ} \mathrm{C}$. Lines are fits to the experimental data using the diffusion limited precipitation model presented in the next section. 
Sources of errer in the recovery transient

The recovery transient for the undoped layer is replotted as a loglog plot in Fig. 3.9 (b). I have included it to separate the recovery transients for each annealing temperature and show the sources of error in the measurement. The error bars indicate a fixed error of $\pm 3 \%$ in measuring the relative $I R$ absorption at a wavelength of $\lambda=1 \mu \mathrm{m}$. Any error in our calibration of the absorbance to determine the defect concentration (errors could be incorrect cross section or sample thickness) have been eliminated by normalizing the concentration. This error becomes a more significant fraction of the concentration as the anneal progresses and the concentration drops. The x-error bars represent the uncertainty in the time of the anneal. The primary source of error in time is related to the uncertainty in the heating and cooling rates of the sample. The sample must be inserted and removed from the furnace for each IR absorption measurement. Without a thermocouple attached directly to the sample, the heating and cooling rate error can only be roughly estimated to be on the order of 1 minute per heating/cooling cycle. This error compounds itself with each successive measurement. One sees from Fig. 3.9(b) that errors in time can be significant at the beginning of the anneal while errors in measuring the concentration can be significant towards the end of the anneal.

Doped layers

Figures 3.10 and 3.11 similarly show the time dependence of the concentration of AsGa-related defects for the Be- and Si-doped layers. Figures 3.10 (a) and (b) are semilog plots for $[\mathrm{Be}]=10^{16}$ and $[B e]=10^{18} \mathrm{~cm}^{-3}$ doped layers respectively. The log of the defect 

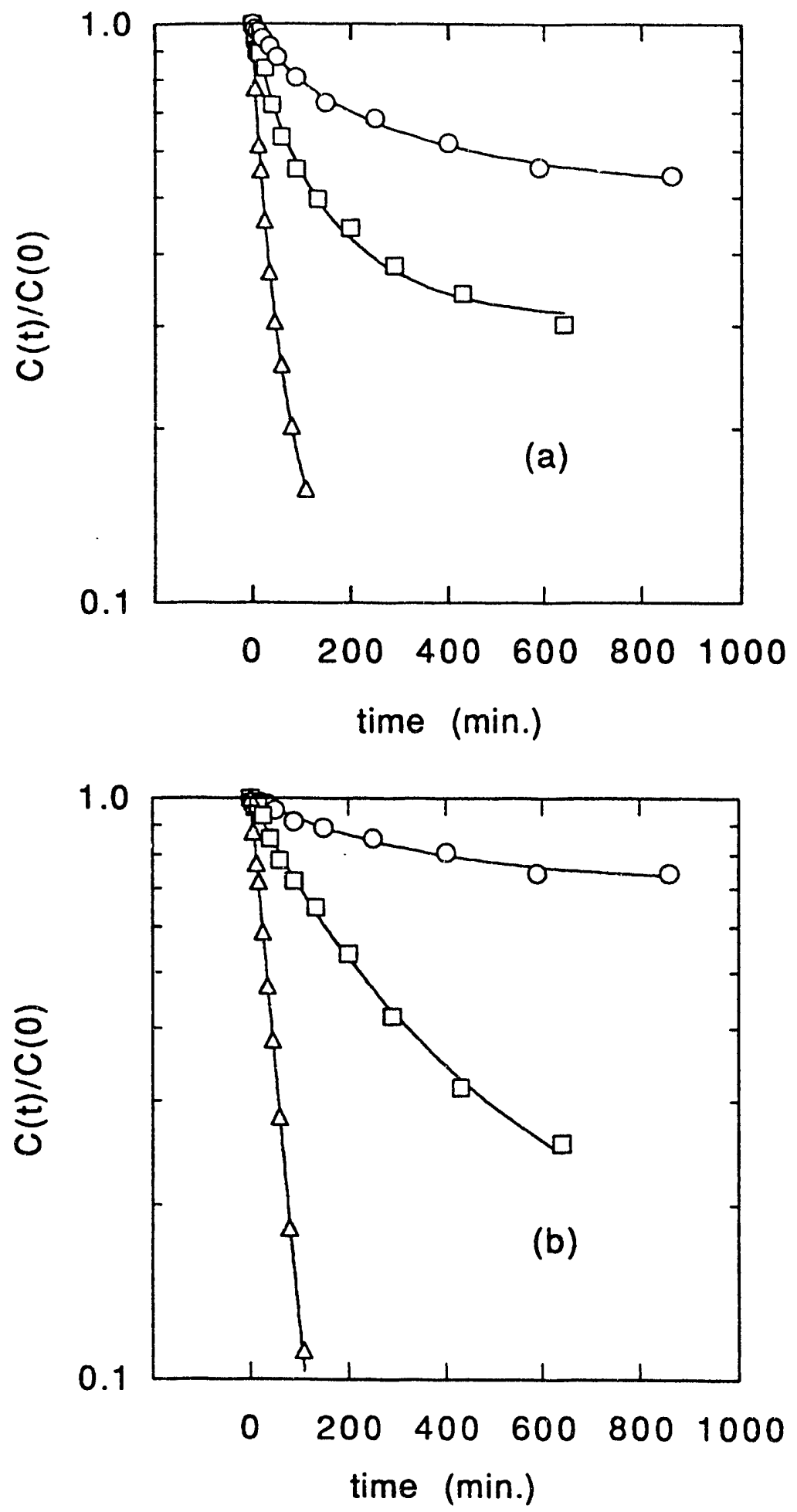

FIG. 3.10 Semi-log plot of the normalized concentration of AsGa-related defects versus isothermal annealing time for the Be-doped LTMBE GaAs layers. (a) $[\mathrm{Be}]=10^{16} \mathrm{~cm}^{-3}$ (b) $[\mathrm{Be}]=10^{18} \mathrm{~cm}^{-3}$. Annealing temperatures are: $0420^{\circ} \mathrm{C}, \square 450^{\circ} \mathrm{C}, \Delta 490^{\circ} \mathrm{C}$. Lines are fits to the experimental data using the diffusion limited precipitation model presented in the next section. 
concentration is shown on the y-axis. The solid lines are the fits to the annealing model to be discussed in the following section. Again, at low temperature the defect concentration saturates and does not tend to zero.

To check the stability of the residual AsGa-related defects after the isothermal annealing at $420{ }^{\circ} \mathrm{C}$, the $[\mathrm{Be}]=10^{16} \mathrm{~cm}^{-3}$ was re-annealed at $490{ }^{\circ} \mathrm{C}$. After 60 minutes of re-annealing at the higher temperature, the concentration of AsGa-related defects was reduced by only $10 \%$. This indicates that the annealing process depends on the past history of the sample. Had the annealing process not shown hysteresis, the concentration should have been reduced by $90 \%$ by re-annealing. The lower temperature anneals are able to stabilize the defects.

Figures 3.11 (a) and (b) are semilog plots of the isothermal annealing transients for the $[S i]=10^{17}$ and $[S i]=10^{19} \mathrm{~cm}^{-3}$ doped layers at the three annealing temperatures. The Si-doped layers also show a saturation of the annealing at low temperatures. In addition, another feature of the recovery transient, different from the undoped or Be-doped layers, is the presence of a much faster initial recovery. This is particularly apparent in the $[S i]=10^{19} \mathrm{~cm}^{-3}$ doped sample (see Fig 3.11b). The AsGa-related defect concentration decreases much more rapidly than for the $\mathrm{Be}$ - or undoped sample. Also the concentration at which the annealing saturates at low temperature is a much smaller fraction of the total defect concentration. The fast initial transient is due to the presence of a high concentration of less stable NPQ defects. 

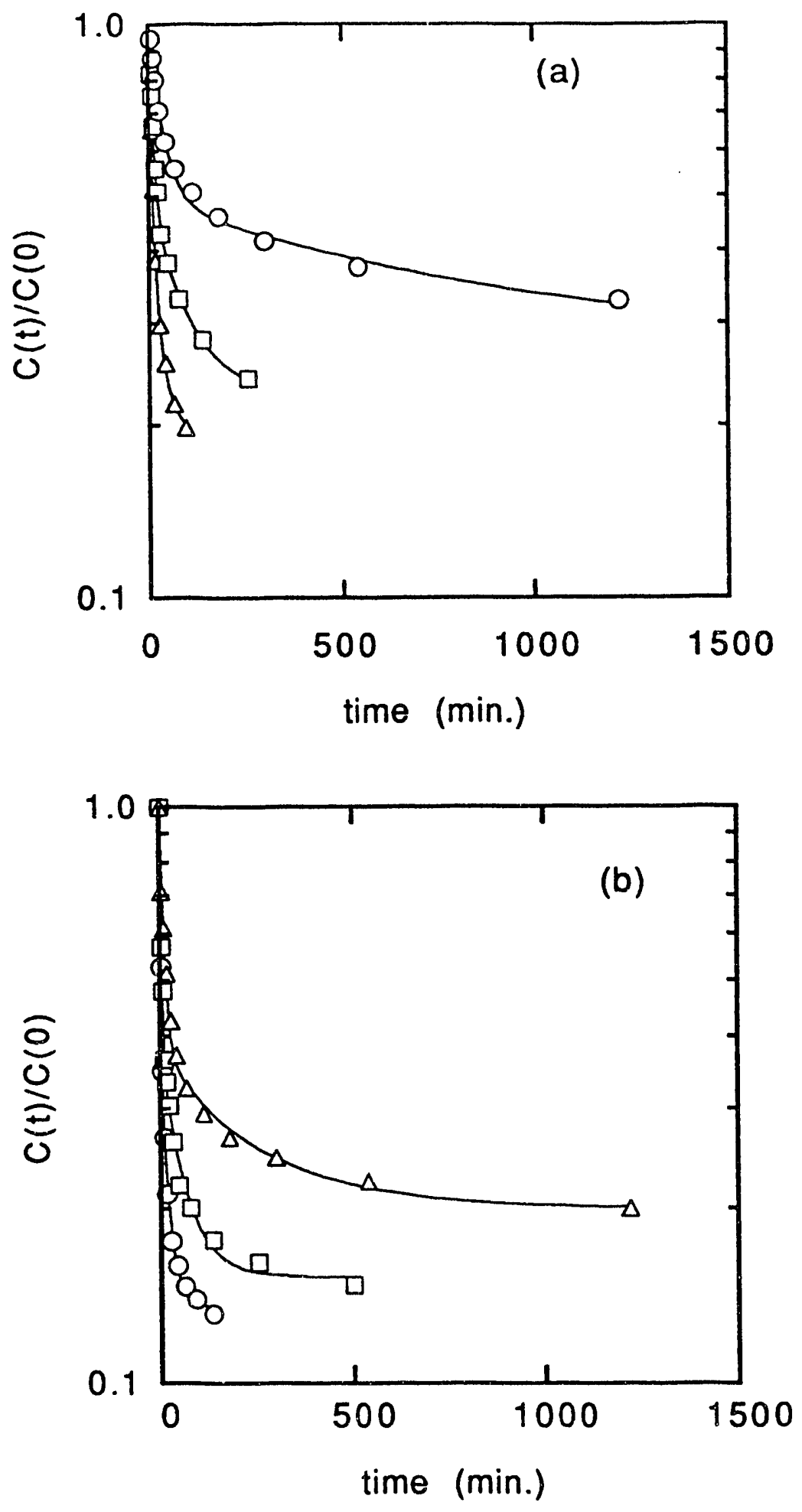

FIG. 3.11 Semi-log plot of the normalized concentration of AsGa-related defects versus isothermal annealing time for the Si-doped LTMBE GaAs layers. (a) $[\mathrm{Si}]=10^{17} \mathrm{~cm}^{-3}$ (b) $[\mathrm{Si}]=10^{19} \mathrm{~cm}^{-3}$. Annealing temperatures are: $0400^{\circ} \mathrm{C}$, $\square 440^{\circ} \mathrm{C}, \Delta 480^{\circ} \mathrm{C}$. Lines are fits to the experimental data using the diffusion limited precipitation model presented in the next section. 
3.3.3. Model of $V_{G a}$ enhanced diffusion of $A s_{G a}$ to precipitates

Constant diffusivity

In this section we show that the annealing characteristics of the PQ AsGa-related defects in LTMBE GaAs can be described in terms of time dependent gallium vacancy enhanced diffusion of AsGa to arsenic precipitates. As has been shown in the previous section, we find that in LTMBE GaAs most of the AsGa-related defects anneal very rapidly at about $500^{\circ} \mathrm{C}$. This behavior is similar to the annealing characteristics of EL2 in GaAs heavily damaged by plastic deformation (Weber and Schneider 1983), or by neutron irradiation (Coates and Mitchell 1975). In stark contrast, EL2 defects in asgrown bulk GaAs crystals are much more stable and anneal away only at temperatures higher than $1000^{\circ} \mathrm{C}$. The difference in the annealing behavior has led to suggestions that the EL2-like defects in heavily damaged GaAs are defect complexes rather than isolated AsGa defects. We would like to argue here that this may not be the only possibility.

Our positron annihilation results indicate the presence of excess $V_{G a}$ in LTMBE (Wei, et al. 1992). Also, it has been shown that large concentrations of $V_{G a}$ exist in neutron irradiated GaAs (Dlubek and Kruse 1987, see e.g. Fig. 14). Therefore, we propose generally for the class of damaged GaAs materials containing excess $V_{G a}$ that a low temperature $\left(\sim 500^{\circ} \mathrm{C}\right)$ annealing of AsGa-related defects is enhanced by the presence of $V_{G a}$ acceptors. Mobile $V_{G a}$ assist the diffusion of AsGa. The diffusion progresses as a site exchange process 


$$
A s_{G_{1}}+V_{G_{2}} \Leftrightarrow V_{G_{1}}+A s_{G_{2}}
$$

where the subscripts 1 and 2 denote the two neighboring $\mathrm{Ga}$ sublattice sites. The specific details of the actual mechanism for the exchange of an atom and vacancy over 2 nearest neighbor distances is open to question. One can imagine mechanisms in which the next nearest neighbor atom moves interstitially to the vacancy or utilizes the intermediate nearest neighbor As site. Another possible mechanism involves the simultaneous concerted exchange of 4 atoms in the vicinity of the vacancy to produce a vacancy hop. What the specific microscopic mechanism might be, however, is not a concern of the present work. This experiment does not give the exact details of the diffusion mechanism but rather information on

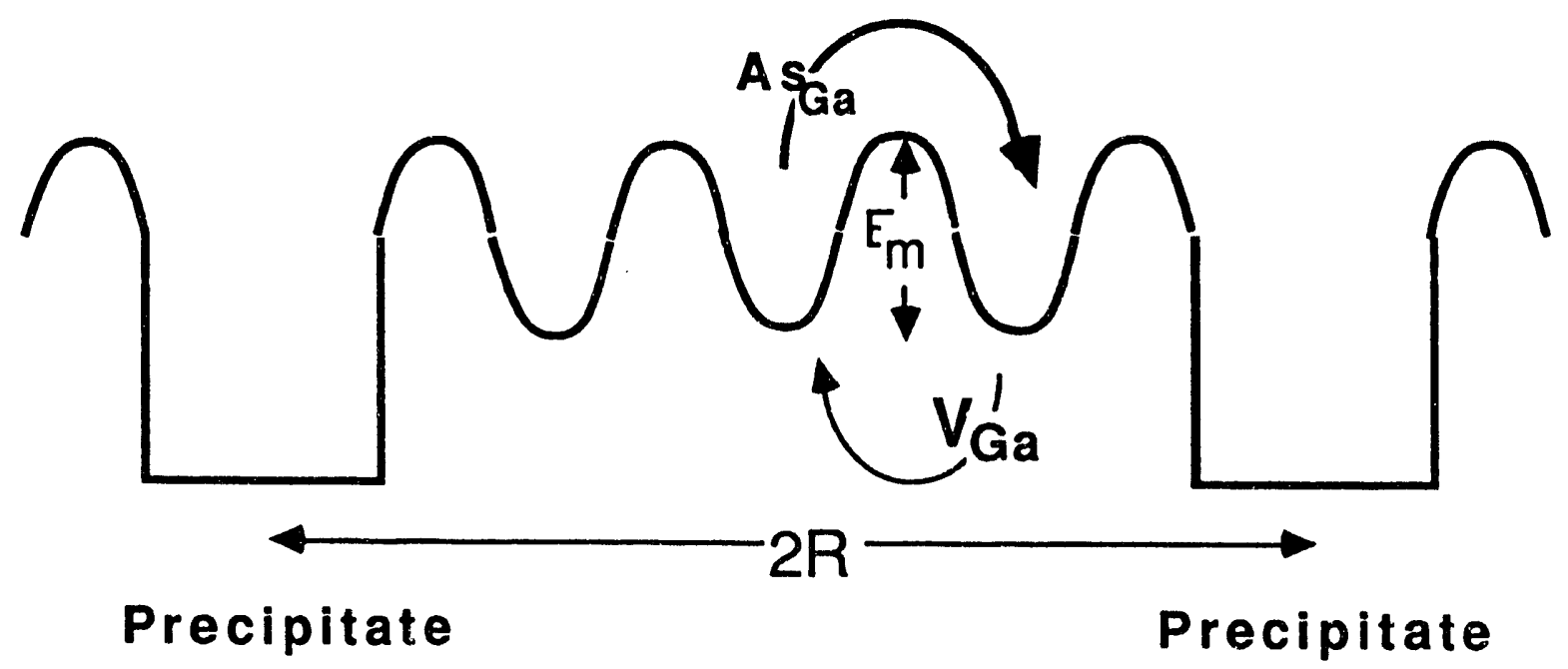

FIG. 3.12 Schematic energy diagram of $V_{G a}$ enhanced diffusion of $A s_{G a}$ to arsenic precipitates. $E_{m}$ is the migration energy of $A s_{G a}$ defect . 
the overall energetics of the diffusion limited annealing process. Without advocating any particular site exchange mechanism, it is sufficient to claim that the mobile $V_{G a}$ in LTMBE GaAs facilitate the diffusion of AsGa defects towards precipitates acting as As sinks. Since the $V_{G a}$ already exist in the material, only the energy of migration, $E_{m}$ must be overcome for $A s G a$ diffusion to occur. $A$ schematic representation of the energetic process to overcome the migration energy is shown in Fig. 3.12.

In contrast to LTMBE GaAs layers, the concentration of $V_{G a}$ in bulk GaAs grown under equilibrium conditions is quite low. For diffusion to occur in these materials, $V_{G a}$ must be created first. Theoretical calculations (Zhang and Northrup 1991) estimate the [V $\mathrm{Va}_{\mathrm{G}}$ ] in stoichiometric undoped $\mathrm{SI}$ material to be around $5 \times 10^{14}$ $\mathrm{cm}^{-3}$. Both the energy of migration, $E_{m}$, and now the formation energy, $E_{f v}$ for $V_{G a}$ must be overcome for diffusion to occur. The value for the enthalpy of formation of $V_{G a}$ in As rich SI GaAs given by Zhang and Northrup (1991) is $\Delta H_{f v}=2.5 \pm 0.5 \mathrm{eV}$. If we assume the activation energy overcome for the annealing of AsGa-related defects during the isochronal anneal is the migration energy, then using the rule of thumb the $450{ }^{\circ} \mathrm{C}$ annealing stage corresponds to $E_{m}$ $=1.8 \pm 0.5 \mathrm{eV}$. The sum, ignoring an entropy term, gives an activation energy for diffusion of AsGa in bulk GaAs of roughly $4.3 \pm 1 \mathrm{pl}$. Therefore the diffusion of AsGa in bulk material would require an annealing temperature on the order of the melting point, much higher than for damaged materials which already contain excess $V_{G a}$. This is observed experimentally. EL2, the As-related defect in bulk materials is very stable up to temperatures of $1100^{\circ} \mathrm{C}$. 
To better understand the diffusion kinetics, I have developed a semi-empirical diffusion model describing $V_{G a}$ assisted diffusion of AsGa to As precipitates. Given experimentally known quantities about the size and concentration of precipitates as well as the concentration of AsGa-related defects, the model describes the dependence of the AsGa concentration on the concentration of $V_{G a}$. The main result of the model is the determination of activation enthalpies for the diffusion of $A s G_{a}$ and $V_{G a}$. It also estimates the concentration of $V_{G a}$ necessary to promote diffusion and the rate at which $V_{G a}$ are lost from the crystal. The essence of the model is to solve the spherically symmetric problem of $A s G$ a diffusion to a finite sized precipitate acting as an infinite sink.

Consider first how $V_{G a}$ can influence the diffusivity of $A s G a$.

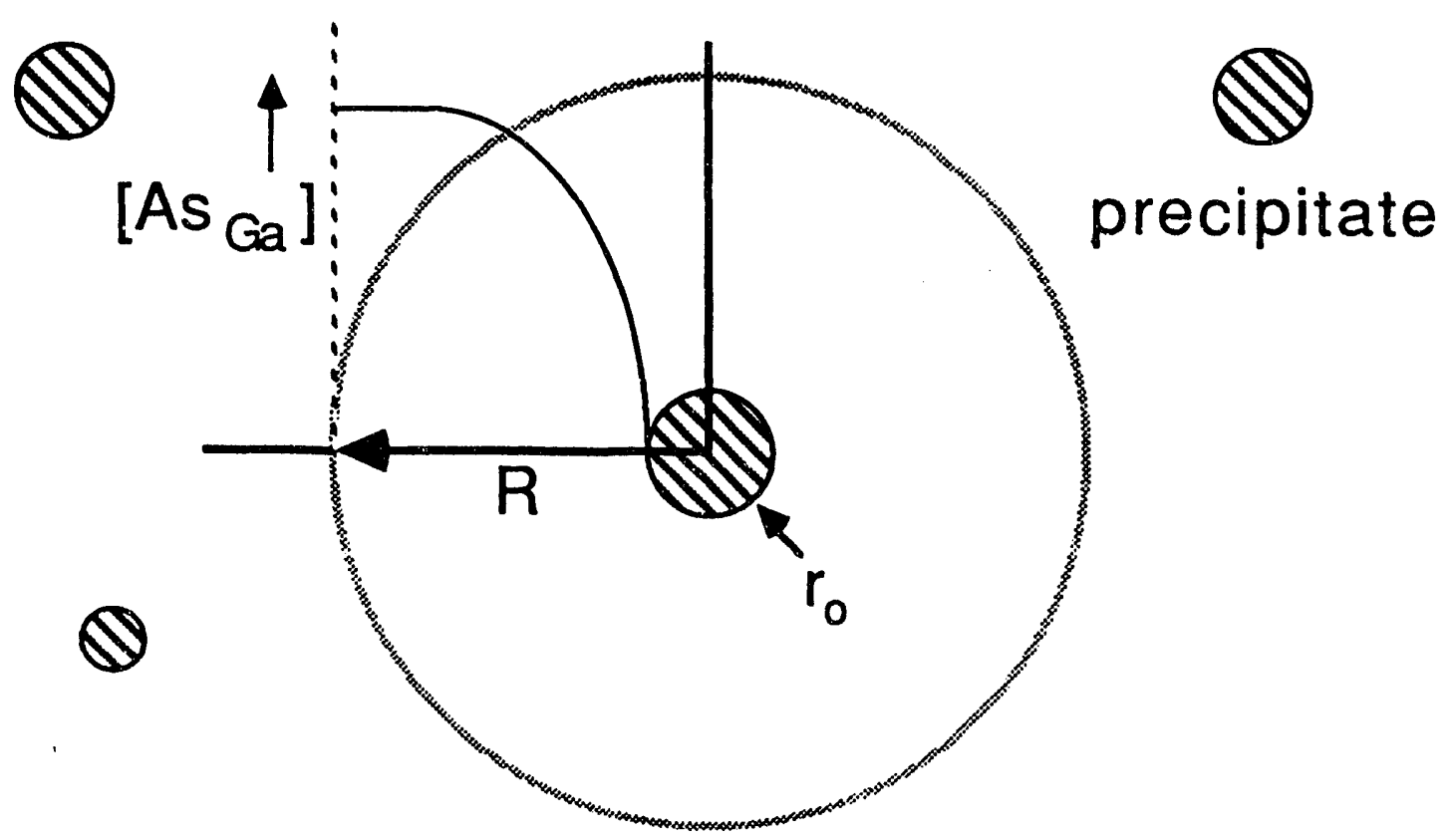

FIG. 3.13 Schematic representation of diffusion problem solved for the annealing of AsGa-related defects. Shown are arsenic precipitates with a mean radius $r_{0}$ and a mean inter-separation $2 R$. A diffusion profile is superimposed on the sphere influenced by the precipitate at the center. 
For the zinc blende lattice, where the $A s G$ a jumps from $\mathrm{Ga}$ site to $\mathrm{Ga}$ site the diffusivity can be expressed as

$$
D=\frac{1}{6} a_{0}^{2} \Gamma_{j}
$$

where $a_{o}$ is the lattice parameter and $\Gamma_{j}$ is jump frequency. The jump frequency can be expressed as the product of three quantities: 1) The probability an $A s_{G a}$ has sufficient energy at temperature $T$ to overcome the barrier to migration, $\mathrm{E}_{\mathrm{m}}=\Delta \mathrm{H}_{\mathrm{n}}-\mathrm{T} \Delta \mathrm{S}_{\mathrm{m}}$, where $\Delta \mathrm{H}_{\mathrm{m}}$ and $\Delta S_{\mathrm{m}}$ are the enthalpy and entropy of migration, 2) The probability a neighboring site is occupied by a vacancy, $x_{V}=\left[V_{G a}\right] /[G a$ sites $]$, and 3) The attempt frequency, taken as the highest optical phonon frequency, $v_{0}=8 \times 10^{12} \mathrm{~s}^{-1}$. Thus, the diffusivity is directly proportional to the concentration of $V_{G a}$ as given by 2). The jump frequency is then,

$$
\Gamma_{j}=x_{v} v_{o} \exp \left(\frac{\Delta S_{m}}{k}\right) \exp \left(-\frac{\Delta H_{m}}{k T}\right) .
$$

The problem is now to solve the spherically symmetric diffusion equation. Figure 3.13 shows a schematic representation of this diffusion problem. The mean radius of the precipitates is $r_{0}$. The diffusion volume is the sphere of influence around a precipitate of radius $R$ which is defined as

$$
\frac{4}{3} \pi R^{3} C_{p}=1
$$


This definition implies that the entire volume of the crystal is accounted for so that all the antisites can diffuse to an arsenic precipitate. Given these conditions, solutions for the average concentration as a function of time are derived in appendix $B$ and have the form

$$
\bar{C}(t)=C_{0} \exp \left(-D \lambda_{n}^{2} t\right),
$$

where $D$ is the diffusivity and $\lambda_{n}$ is a characteristic inverse length parameter derived from the geometry of the problem and boundary conditions (see Appendix B). The time constant for the exponential decay would be,

$$
\tau=1 / D \lambda_{1}{ }^{2} .
$$

\section{Time dependent diffusivity}

The experimental annealing transients are not simple exponentials as seen in Fig's. 3.9 - 3.11. The solution in Eq. 3.7 assumes a constant diffusivity which obviously does not apply. The diffusivity of antisites is proportional to the $V_{G a}$ concentration which is no: constant. Like the concentration of AsGa-related defects, the concentration of $V_{G a}$ is also supersaturated and will recover towards an equilibrium concentration. Therefore, both the $V_{G a}$ concentration and hence the diffusivity are functions of time. Ideally we would like to be able to monitor the $V_{G a}$ concentration during the isothermal annealing. Unfortunately, it would be extremely difficult if not impossible at this point, to measure the $V_{G a}$ concentration as a function of time. Positron annihilation 
experiments are very time consuming and only semi-quantitative at best. Therefore we will assume an exponential time dependence for the $V_{G a}$ concentration and hence diffusivity,

$$
\begin{aligned}
& {\left[V_{G a}\right]=V_{G_{a}}^{0} \exp \left[\frac{-t}{\tau_{v}}\right],} \\
& D=D_{v}^{0} \exp \left[\frac{-t}{\tau_{v}}\right],
\end{aligned}
$$

where $\tau_{v}$ is the time constant for the loss of $V_{G a}$ diffusing to vacancy sinks. These could be for example voids (vacancy precipitates), defect complexes or interfaces which include the sample surface, the layer interface and the arsenic precipitate interface. Implicit in this assumption is that the spatial variation of the $V_{G a}$ concentration is not correlated to the AsGa concentration. In other words the $V_{G a}$ concentration can be treated as spatially uniform and therefore depends only on time. Solutions to the diffusion equation (B.1) including the time dependent diffusivity (Eq. 3.10) have been derived in appendix $B$. The solutions for the average concentration of AsGa-related defects take the form of an exponential of an exponential,

$$
\bar{C}(t)=C_{0} \exp \left(\frac{-\tau_{v}}{\tau_{A s}}\right) \exp \left[\frac{\tau_{v}}{\tau_{\text {As }}} \exp \left(\frac{-t}{\tau_{v}}\right)\right] .
$$

Normalizing the concentration and taking the logarithm, as the data are plotted in Fig. 3.9, Eq. 3.11 becomes an exponential with an offset, 


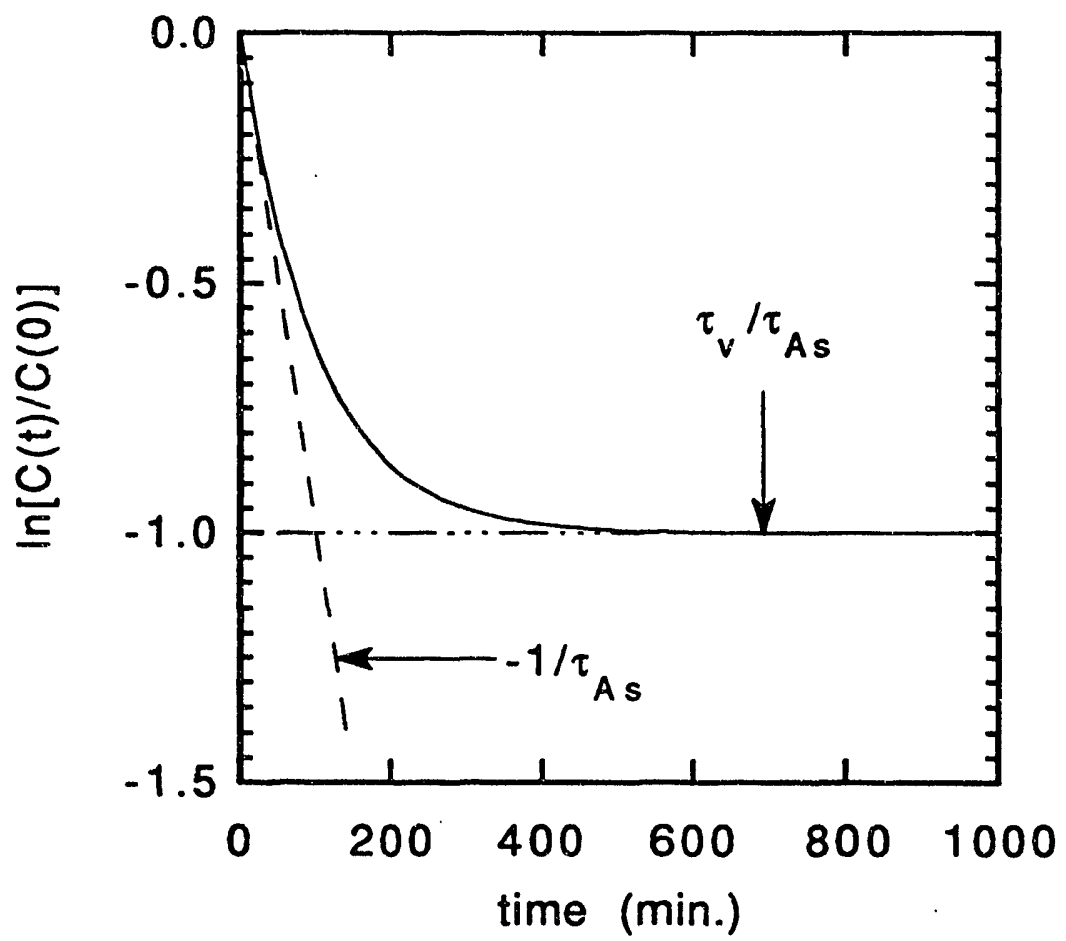

FIG. 3.14 Semilog plot of Eq. 3.11 describing the average AsGa concentration of a diffusion limited precipitation problem with an exponentially time dependent diffusivity. Limiting behavior is indicated by the dashed lines. $\tau_{v}=\tau_{A s}=100 \mathrm{~min}$.

$$
\ln \left(\frac{\bar{C}(t)}{C_{0}}\right)=\frac{\tau_{v}}{\tau_{A s}}\left[\exp \left(\frac{-t}{\tau_{v}}\right)-1\right]
$$

The limits of Eq. 3.12 at short times and infinite time are informative. For $t \ll \tau_{v}$, the exponential can be approximated by $\exp \left(-t / \tau_{v}\right) \approx 1-t / \tau_{v}$ and the AsGa concentration is,

$$
\bar{C}(t) \approx C_{0} \exp \left(\frac{-t}{\tau_{A s}}\right) \text {. }
$$

which is the same as Eq. 3.7 for a constant diffusivity. Thus for $t \ll \tau_{v}$, the initial slope of a semilog plot of the AsGa-related defect concentration is $-1 / \tau_{\mathrm{As}}$ as shown in Fig. 3.14. In the other extreme, 
the limit of Eq. 3.12 as $t \rightarrow \infty$ is just $\tau_{v} / \tau_{A s}$. If $\tau_{v} \gg \tau_{A s}$, the limit of very long lived vacancies, then Eq. 3.12 again becomes the same as Eq. 3.7 for a constant, time independent diffusivity.

\section{Fitting the diffusion model to the data}

One additional correction to Eq. 3.11 must be made before all the annealing transients can be accurately described for all times. The fast initial transient of the less stable NPQ must be accounted for, particularly for the Si-doped samples which contain a large fraction of NPQ defects. Initially, the defect recovery shows a larger and much faster transient before saturating than Eq. 3.11 predicts. As seen in the isochronal anneals (see Fig. 3.11) the NPQ defects are less stable than the $P Q$ defects. Since we can not distinguish between NPQ and $P Q$ defects at room temperature the recovery transient will be the sum of both responses. However, the initial fraction of NPQ defects is known from the low temperature IR absorption measurements. This fact can be used account for the effect of the NPQ defects. By including a fast exponential recovery of the NPQ fraction of defects in parallel with the PQ fraction, Eq. 3.11 is modified so that the average AsGa-related defect concentration is

$$
\bar{C}(t)=C_{0}\left\{(1-f) \exp \left(\frac{-\tau}{\tau_{\mathrm{NPQ}}}\right)+(f) \exp \left(\frac{-\tau_{v}}{\tau_{\mathrm{As}}}\right) \exp \left[\frac{\tau_{v}}{\tau_{\mathrm{As}}} \exp \left(\frac{-t}{\tau_{v}}\right)\right]\right\},
$$

where $f$ is the fraction of PQ defects, and $\tau_{N P Q}$ is the time constant for the annealing of the NPQ defects. This modification introduces one more free parameter, $\tau_{\mathrm{NPQ}}$. However, $\tau_{\mathrm{NPQ}} \ll \tau_{\mathrm{As}}$ or $\tau_{\mathrm{v}}$. Hence it 
does not mix strongly with $\tau_{A s}$ or $\tau_{v}$ and only slightly weakens the confidence in determining $\tau_{A s}$ and $\tau_{v}$ accurately. Actually, $\tau_{A s}$ and $\tau_{v}$ are quite sensitive to the choice of $f$. Fits of Eq. 3.14 to the data for the Be-doped, undoped and Si-doped layers are shown in Fig's. 3.9, 3.10 and 3.11. One can see that Eq. 3.14 describes all the annealing curves exceedingly well for the entire annealing time. I am encouraged that this is a proper treatment of the annealing transients by the fact that if $f$ is allowed to become a free parameter also, a Chi-squared fitting routine settles in on a value very close to that determined by the low temperature IR absorption photoquenching experiment. The values of $f$ determined by the fit are typically within $3-5 \%$ of the IR absorption values at the lowest annealing temperature. The fit to the defect recovery at the lowest annealing temperature is the most sensitive to the choice of $f$. This is because at the lowest annealing temperature as $t \rightarrow \infty$, the saturation concentration of defects is most clearly defined (see Fig. $3.9 a$ for instance). On a log scale, the final saturation concentration, $C_{f}$, in the limit as $t \rightarrow \infty$ is now

$$
\ln \left(\frac{C_{f}}{f C_{0}}\right)=\frac{\tau_{v}}{\tau_{A s}}
$$

If $f$ is not chosen properly the fit misses badly in this limit. For the two higher annealing temperatures, the fitting procedure was run with $f$ fixed at the value which had given the lowest Chi-squared at the lowest annealing temperature.

This method of choosing $f$ is crucial to obtaining meaningful results from the fitting process. Later on, when determining 
activation enthalpies, the values can vary by as much as $\pm 0.5 \mathrm{eV}$ due to a $10 \%$ change in the choice of $\mathrm{f}$. Determinations of the photoquenchable fraction by photobleaching with white light at liquid $\mathrm{He}$ temperatures are typically reproducible to only $\pm 5-10 \%$ and could therefore introduce a significant error in determining the activation enthalpy. What we see is that the annealing recovery is a sensitive measure of the photoquenchable fraction of neutral defects to within $\pm 1 \%$.

The isothermal annealing of the AsGa-related defects in all the LTMBE GaAs layers has been analyzed using Eq. 3.14. The lines in Fig's. 3.9, 3.10, and 3.11 are best fits to the data using Eq. 3.14. Table 3.4 summarizes all the values for $t, \tau_{A s}, \tau_{v}$ and $\tau_{N P Q}$ determined by the Chi-squared non-linear least squares fit to the data. The corresponding activation enthalpies and pre-exponentials determined from an Arrhenius plot (see Fig's. 3.15 and 3.16) of the time constant parameters is also included in Table 3.4.

Electronic energy level of NPQ AsGa-related defect

As an aside, the fact that IR absorption measurements are sensitive to AsGa-related defects in the neutral charge state is important in the following context. For the $10^{18}$ and $10^{19} \mathrm{~cm}^{-3} \mathrm{Be}-$ doped samples $f=1.0$. This suggests that the electronic level for the NPQ defects lies above that for the $P Q$ defects. The $B e$ has been shown to be electrically active, but compensated in the as-grown layers. "The concentration of NPQ defects in the Be-doped layers is on the order of $10^{18} \mathrm{~cm}^{-3}$. The fact that we can no longer detect the NPQ defects when the Be-doping is greater than $1018 \mathrm{~cm}^{-3}$ indicates that the NPQ defects are being compensated by the Be before the PQ 
defects. For this to occur the NPQ defect electronic level must lie above the $P Q$ defect level.

TABLE 3.4 Summary of isothermal annealing time constants of AsGa-related defects in LTMBE GaAs. Values of the parameters $\tau_{A s}, \tau_{v}, \tau_{\mathrm{NPQ}}$ are determined by a Chi-squared, non-linear, least squares fit of the data to Eq. 3.14. The corresponding pre-exponential, $\tau_{0}$, and activation enthalpy $E_{a}$ were obtained from the Arrhenius plots in Fig's. 3.15 and 3.16.

\begin{tabular}{|c|c|c|c|c|c|c|}
\hline \multirow{2}{*}{\multicolumn{2}{|c|}{$\begin{array}{l}\text { Be Doping } \\
\left(\mathrm{cm}^{-3}\right)\end{array}$}} & \multicolumn{3}{|c|}{ Time Constants (min.) } & \multirow{2}{*}{$\begin{array}{c}\tau_{0} \\
(\min .)\end{array}$} & \multirow{2}{*}{$\begin{array}{c}E_{a} \\
\pm 0.3 \mathrm{eV})\end{array}$} \\
\hline & & \multirow{2}{*}{$\frac{420^{\circ} \mathrm{C}}{859}$} & \multirow{2}{*}{$\frac{450^{\circ} \mathrm{C}}{204}$} & \multirow{2}{*}{$\frac{490^{\circ} \mathrm{C}}{33.4}$} & & \\
\hline$[\mathrm{Be}]=10^{16}$ & $\tau_{A s}$ & & & & $3.5 \times 10^{-13}$ & 2.1 \\
\hline \multirow{2}{*}{$f=0.86$} & $\tau_{v}$ & 543 & 267 & 72.7 & $1.5 \times 10^{-7}$ & 1.3 \\
\hline & $\tau_{\mathrm{NPQ}}$ & 60.5 & 22.2 & $3.1+$ & $1.9 \times 10^{-9}$ & 1.4 \\
\hline \multirow{3}{*}{$\begin{array}{c}{[\mathrm{Be}]=10^{17}} \\
f=0.81\end{array}$} & $\tau_{A s}$ & 822 & 168 & 32.1 & $3.8 \times 10^{-13}$ & 2.1 \\
\hline & $\tau_{v}$ & 485 & 209 & 65.8 & $1.7 \times 10^{-7}$ & 1.3 \\
\hline & $\tau_{\mathrm{NPO}}$ & $\begin{array}{r}43.4 \\
\end{array}$ & 23.3 & $3.1 t$ & $1.3 \times 10^{-4}$ & 0.9 \\
\hline \multirow{2}{*}{$\begin{array}{c}{[\mathrm{Be}]=10^{18}} \\
f=1.0\end{array}$} & $\tau_{A s}$ & 1366 & 272 & 44.8 & $7.6 \times 10^{-14}$ & 2.2 \\
\hline & $\tau_{v}$ & 636 & 614 & 516 & $6.5 \times 10^{1}$ & 0.1 \\
\hline \multirow{2}{*}{$\begin{array}{c}{[\mathrm{Be}]=10^{19}} \\
f=1.0\end{array}$} & $\tau_{A S}$ & 1093 & 258 & 37.5 & $1.2 \times 10^{-13}$ & 2.2 \\
\hline & $\tau_{v}$ & 177 & 436 & 95.4 & $1.4 \times 10^{-1}$ & 0.4 \\
\hline \multicolumn{2}{|c|}{$\begin{array}{l}\text { Si Doping } \\
\left(\mathrm{cm}^{-3}\right)\end{array}$} & $\begin{array}{r}\text { Time } \\
400^{\circ} \mathrm{C}\end{array}$ & $\begin{array}{l}\text { onstants } \\
440^{\circ} \mathrm{C}\end{array}$ & $\begin{array}{l}(\min .) \\
480^{\circ} \mathrm{C}\end{array}$ & $\begin{array}{c}\tau_{0} \\
\text { (min.) }\end{array}$ & $\begin{array}{c}E_{a} \\
\pm 0.3 \mathrm{eV}\end{array}$ \\
\hline undoped & $\tau_{A s}$ & 1548 & 234 & 38.4 & $1.3 \times 10^{-12}$ & 2.0 \\
\hline \multirow[t]{2}{*}{$f=0.65$} & $\tau_{v}$ & 741 & 236 & 41.5 & $1.6 \times 10^{-9}$ & 1.6 \\
\hline & $\tau_{\mathrm{NPO}}$ & 51.0 & 21.1 & 10.4 & $1.6 \times 10^{-5}$ & 0.9 \\
\hline$[S i]=10^{17}$ & $\tau_{A S}$ & 1065 & 108 & 27.2 & $8.8 \times 10^{-13}$ & 2.0 \\
\hline \multirow[t]{2}{*}{$f=0.53$} & $\tau_{v}$ & 599 & 90.4 & 27.2 & $1.2 \times 10^{-10}$ & 1.7 \\
\hline & $\tau_{\mathrm{NPO}}$ & 28.9 & 9.4 & 6.3 & $6.0 \times 10^{-8}$ & 1.2 \\
\hline$[S i]=10^{19}$ & $\tau_{A s}$ & 239 & 51.3 & 15.3 & $1.4 \times 10^{-9}$ & 1.6 \\
\hline \multirow{2}{*}{$f=0.42$} & $\tau_{v}$ & 164 & 50.6 & 17.1 & $1.0 \times 10^{-7}$ & 1.4 \\
\hline & $\tau_{\mathrm{NPQ}}$ & 9.2 & $2.8 \dagger$ & $1.5 t$ & $6.2 \times 10^{-9}$ & 1.2 \\
\hline
\end{tabular}

t These values of the time constants are below the resolution limit of the annealing experiment. 


\section{Enthalpy of migration}

The slope of an Arrhenius plot of the time constants $\tau_{A s}$ and $\tau_{v}$ will yield the activation enthalpy for the annealing of $A s_{G a}$ and $V_{G a}$ respectively. Figures 3.15 and 3.16 are Arrhenius plots of the time constants for the Be- and Si-doped samples respectively. Over the range of annealing temperatures, $\tau_{A s}$ and $\tau_{v}$ are comparable in magnitude indicating that the $V_{G a}$ and $A s G a$ anneal away at similar rates. The activation enthalpy for the annealing of AsGa-related defects in LTMBE GaAs is consistently between 2.0 to $2.2 \mathrm{eV}$, with only the activation enthalpy for the $[S i]=10^{19} \mathrm{~cm}^{-3}$ layer falling below this range at $1.6 \mathrm{eV}$. The activation enthalpy of the $V_{G a}$ is somewhat less, ranging typically from 1.3 to $1.7 \mathrm{eV}$, with the exception of the two highest Be doped samples which exhibit an activation enthalpy near zero. The activation enthalpy for the annealing of the NPQ defects is yet lower, about half of that for the $\mathrm{PQ}$ defects ranging from 0.9 to $1.4 \mathrm{eV}$.

The mechanism of an AsGa hop is equivalent to a $V_{G a}$ hop in the opposite direction, in effect, swapping sites. $V_{G a}$ must be present for the AsGa to move. The opposite is not true for $V_{G a}$ diffusion. The $V_{G a}$ does not require $A s_{G a}$. In fact for most hops, the $V_{G a}$ is swapping sites with $\mathrm{Ga}$ atoms. Thus we conclude that the difference in the enthalpies of migration is due to the difference in energy between a As atom and a Ga atom hopping into the vacancy, $\Delta H_{m}^{A s}=2.0 \pm 0.3 e V$ versus $\Delta H_{m}^{v}=1.5 \pm 0.3 \mathrm{eV}$ respectively. It is energetically more favorable for the $V_{G a}$ to swap places with a $G a$ atom than with an $A s$ atom on a $\mathrm{Ga}$ sublattice site. Thus there must be either a slightly 


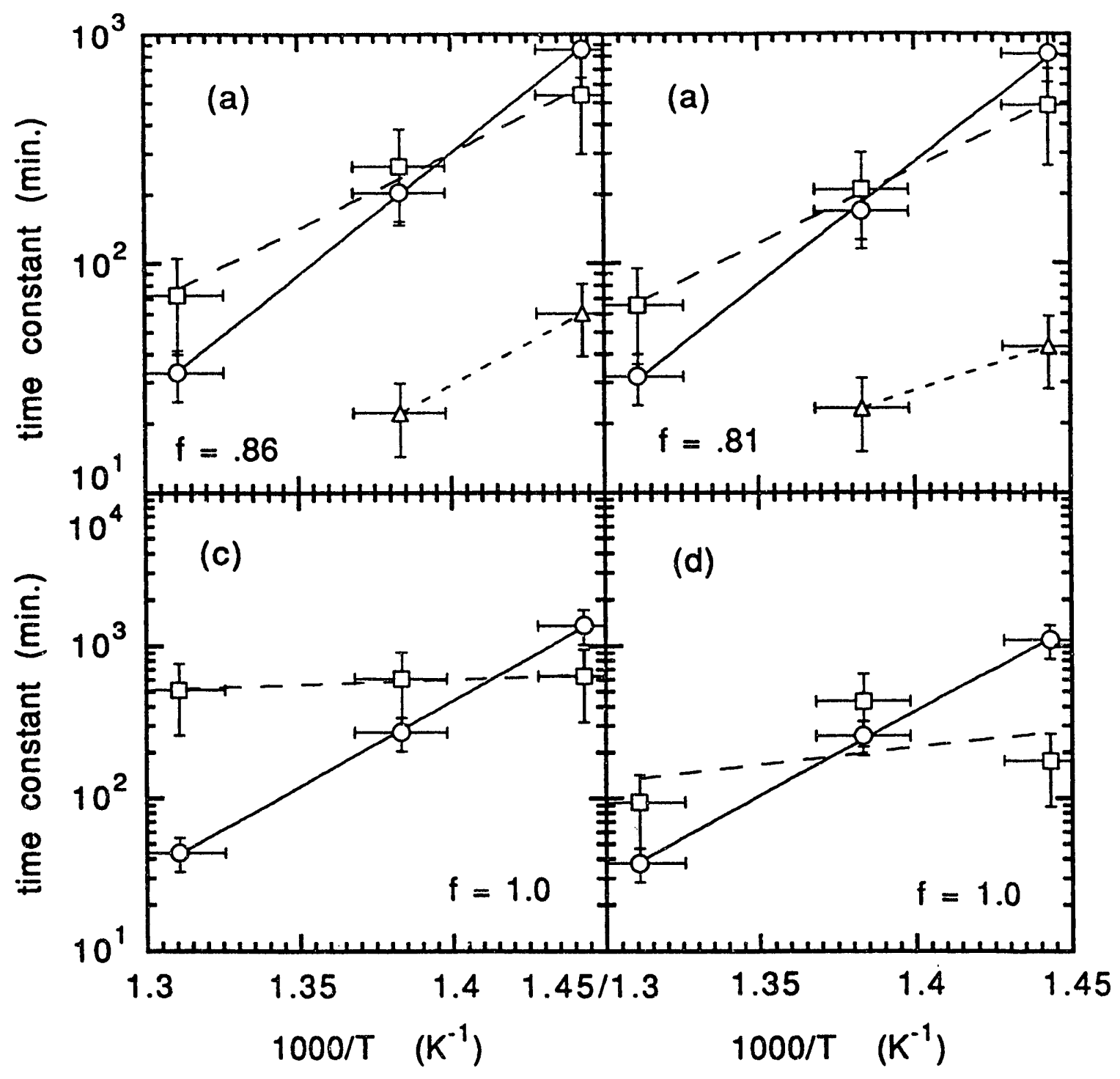

FIG. 3.15 Arrhenius plot of $\tau_{A s}, \tau_{v}, \tau_{\mathrm{NPO}}$ and for the Be-doped layers, determined from the Isothermal annealing transient of the AsGa-related defect concentration. Symbols are the parameters obtained by a Chi-squared fit to Eq. 3.14: (o) $\tau_{A s}$, (D) $\tau_{v},(\Delta) \tau_{N P Q}$. Sample doping is (a) $[B e]=10^{16} \mathrm{~cm}^{-3}$, (b) $[B e]=10^{17} \mathrm{~cm}^{-3}$, (c) $[\mathrm{Be}]=10^{18} \mathrm{~cm}^{-3}$, (d) $[\mathrm{Be}]=10^{19} \mathrm{~cm}^{-3}$. Lines are exponential fits to the parameters. $f$ is the photoquenchable fraction of defects used in the fitting process to get the time constants. The corresponding activation enthalpies are summarized in Table 3.4. 


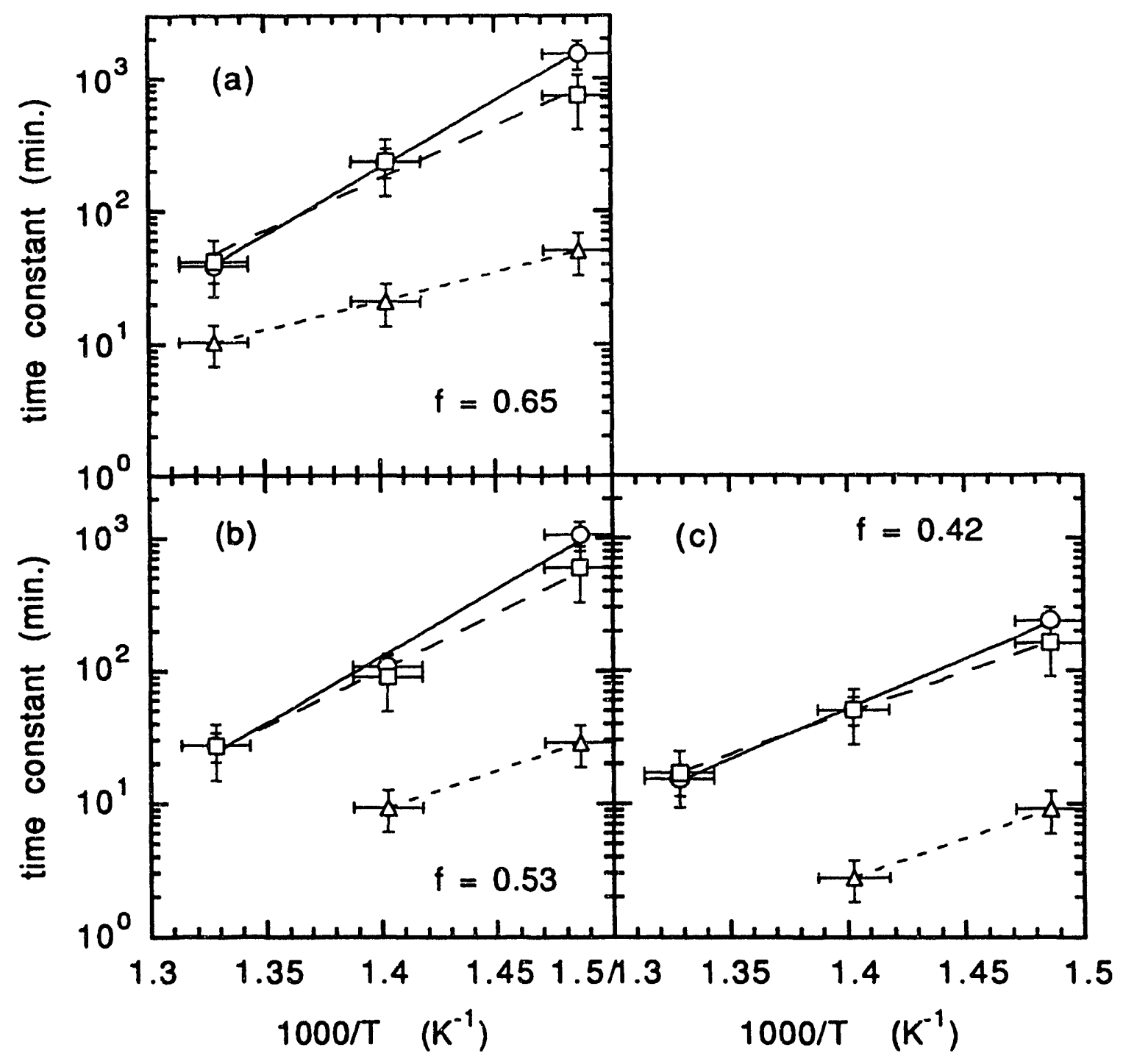

FIG. 3.16 Arrhenius plot of $\tau_{\mathrm{As}}, \tau_{\mathrm{v}}$ and $\tau_{\mathrm{NPQ}}$ for the Si-doped layers, determined from the isothermal annealing transient of the $A s_{G a}$-related defect concentration. Symbols are the parameters obtained by a Chi-squared fit to Eq. 3.14: (o) $\tau_{A s,}(\square) \tau_{v},(\Delta) \tau_{\mathrm{NPQ}}$. Sample doping is (a) undoped (b) $[S i]=10^{17}$ $\mathrm{cm}^{-3}$, (c) [Si] $=10^{19} \mathrm{~cm}^{-3}$. $f$ is the photoquenchable fraction of defects. Lines are exponential fits to the parameters. The corresponding activation enthalpies are summarized in Table 3.4 . 
repulsive or attractive potential between the $A s_{G a}$ and $V_{G a}$ which hinders them from approaching or moving away from each other. It is my opinion that the latter case is true. As isolated defects they both have opposite charge states and opposite strain fields, thereby attracting each other.

This interaction between the defects explains why annealing "soaks" at low temperature for long times stabilize the AsGarelated defects (see section 3.3 .2 on the isothermal annealing transient of the $[\mathrm{Be}]=10^{16} \mathrm{~cm}^{-3}$ sample). At low temperatures, the $V_{G a}$ can still diffuse through the crystal finding sinks, while the temperature is not sufficient to activate the AsGa diffusion. Once the $\mathrm{V}_{\mathrm{Ga}}$ are eliminated, the $A s_{G a}$-related defects can no longer diffuse. Even when the temperature is elevated to $500^{\circ} \mathrm{C}$ after a low temperature soak, which would have significantly reduced the AsGa concentration, very little change in the concentration occurs.

This effect, which I refer to as the "stabilization of AsGarelated defects" could be very important for technological applications of the material, particularly for fast optical switches and pulse generators. In these devices it is important to switch on quickly and also to switch off quickly. High concentrations of defects are required to act as recombination centers for free carriers and switch the device off quickly. However, the need to switch off quickly must be balanced against the ability to hold off large electric fields across the device without excessive leakage current. Hopping conductivity over the defect levels could be a problem if the concentration of defects becomes too great. By 
tuning the annealing temperature and time, the proper concentration of defects could be stabilized for optimum device performance.

Introducing a higher concentration of $\mathrm{Be}$ influences the annealing of the $\mathrm{V}_{\mathrm{Ga}}$ while increased $\mathrm{Si}$ doping influences the annealing of the AsGa. In both cases the effect is to reduce the enthalpies of migration. At this point 1 do not fully understand this effect. It is not necessarily a Fermi level effect since the only defects to change charge states by doping are the NPQ defects.

The activation enthalpy for the annealing of the NPQ AsGarelated defects is $1.1 \pm 0.3 \mathrm{eV}$. It is not clear why it is so low, nearly half that of the $P Q$ defects. Also, it is not possible to stabilize the NPQ defects with low temperature anneals. In Chapter 2, we have argued that the NPQ defects are PQ defects with another defect nearby. The lower activation enthalpy for the annealing of the NPQ defects suggests that it easier for the defect and its neighbor to combine. It is very likely that the resulting defect complex is the nucleus for arsenic precipitation. However, the number of $A s G a$ defects required to form the precipitate nucleus is uncertain but probably lies in the range of 2-10 AsGa. Figure 3.17 shows a schematic representation of the difference in migration enthalpies for this scenario. An attractive interaction between the NPQ defect and the defect or precipitate nucleus could cause an effective lowering of the barrier. The magnitude of an energy field which could cause an $0.9 \mathrm{eV}$ lowering of the barrier would be approximately equal to the energy difference divided by the site spacing or approximately $2 \times 10^{7} \mathrm{eV} \mathrm{cm}^{-1}$. 


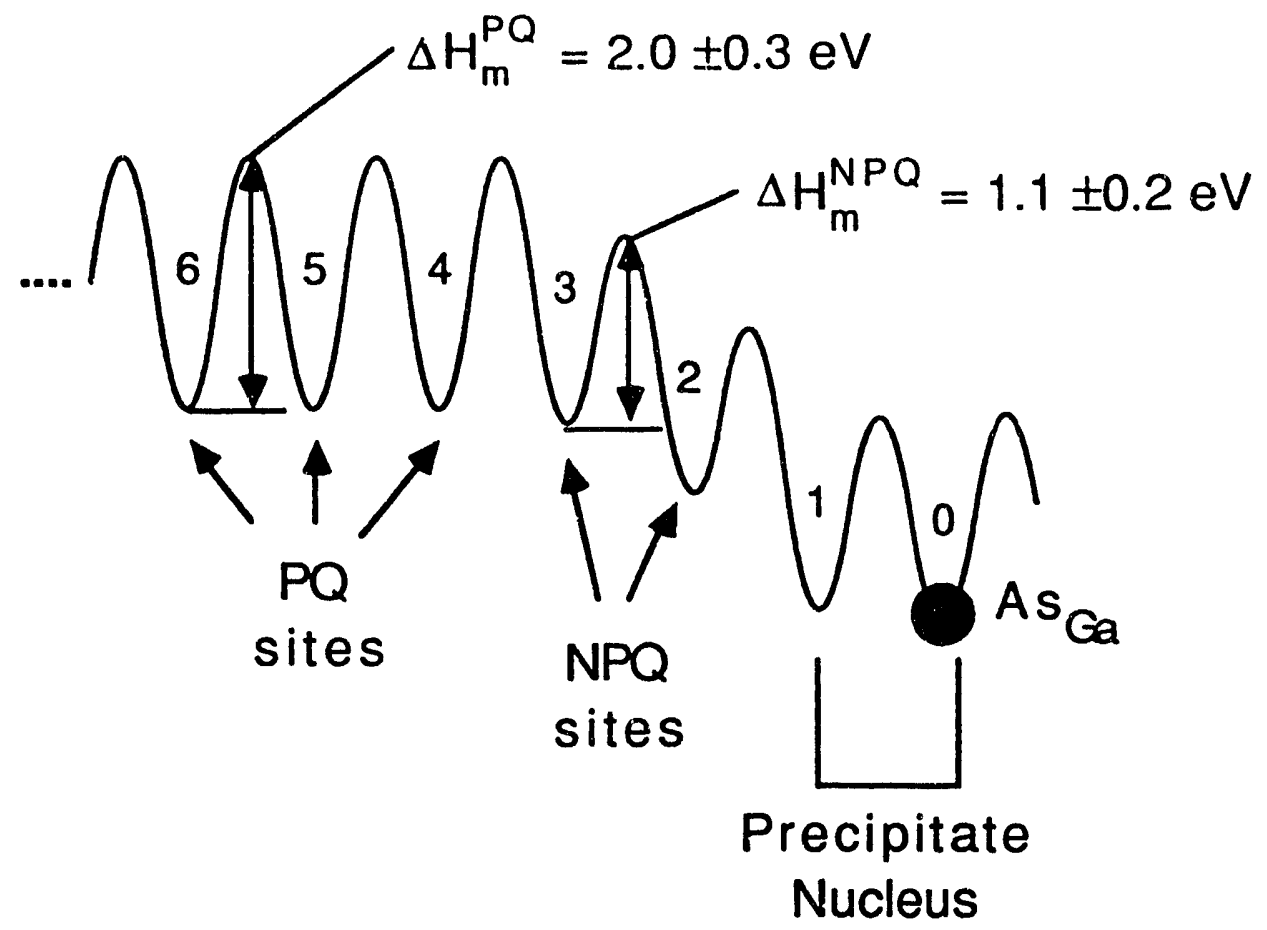

FIG. 3.17 Schematic representation of the possible difference between photoquenchable (PQ) and non-photoquenchable (NPQ) As Ga-related defects. Numerals signify nearest neighbor (n.n.) shells on the Ga-sublattice. NPQ sites are within 3 n.n. shells of another $\mathrm{AsGa}_{\mathrm{Ga}}$ defect or arsenic precipitate. PQ defects lie at distances greater than 3 n.n.

One possible origin for the lowering of the migration barrier could be the interaction of the strain fields of the precipitate nucleus and the NPQ defect. In related studies, the effect of the strain associated with introducing the isovalent impurity indium in GaAs was considered on the formation energy of $V$ (Rytova and Soloveva 1986). In this work the formation energy of $V_{G a}$ was found to be lowered by $1 \mathrm{eV}$ due to the presence of In. Vacancy formation and migration energies are of similar magnitude as both involve the breaking of bonds. If the strain associated with the introduction of In lowered the formation energy of $V_{G a}$ by $1 \mathrm{eV}$, then it does not seem unreasonable that the strain associated with an arsenic precipitate nucleus could likewise reduce the migration energy by 
$0.9 \mathrm{eV}$. The manifestation of the lowering of the $\mathrm{V}_{\mathrm{Ga}}$ migration energy is the lower migration enthalpy of NPQ defects assisted by the $V_{G a}$ near the precipitates.

Overall, I am encouraged by the activation enthalpies found for the annealing of defects in LTMBE GaAs. They compare favorably to activation energies found in other GaAs materials. Activation enthalpies of 1.2 and $1.6 \mathrm{eV}$ were found for the annealing of defects in electron-irradiated GaAs (Farmer and Look 1980). Also an interesting study of $\mathrm{V}_{\mathrm{Ga}}$ enhanced mixing of multilayers (Rouviere, et al. 1992) supports the activation enthalpy for $V_{G a}$ determined in our work. They obtained a similar value for the enthalpy of migration $\left(\Delta H_{m}^{V}=1.7 \mathrm{eV}\right)$ of $V_{G a}$ but by a very different method. By analyzing the depth dependence for the intermixing of an AlGaAs layer in GaAs by TEM, they were able to determine the diffusivity of $V_{G a}$ from the surface. The fact that a similar value has been obtained by a very different technique gives us confidence that the value for the enthalpy of migration is accurate to within a few tenths of an $\mathrm{eV}$.

Concentration dependent diffusion

Although similar activation enthalpies for all the samples are obtained, the time constants of the various samples depend on the AsGa-related defect concentration. To illustrate the concentration dependence of the defect annealing rate, the time constants taken at a temperature of $450^{\circ} \mathrm{C}\left(440^{\circ} \mathrm{C}\right.$ for the Si-doped layers) are plotted versus the EL2-like defect concentration in Fig. 3.18 as a $\log -\log$ plot. Although the concentration of defects does not cover a wide 


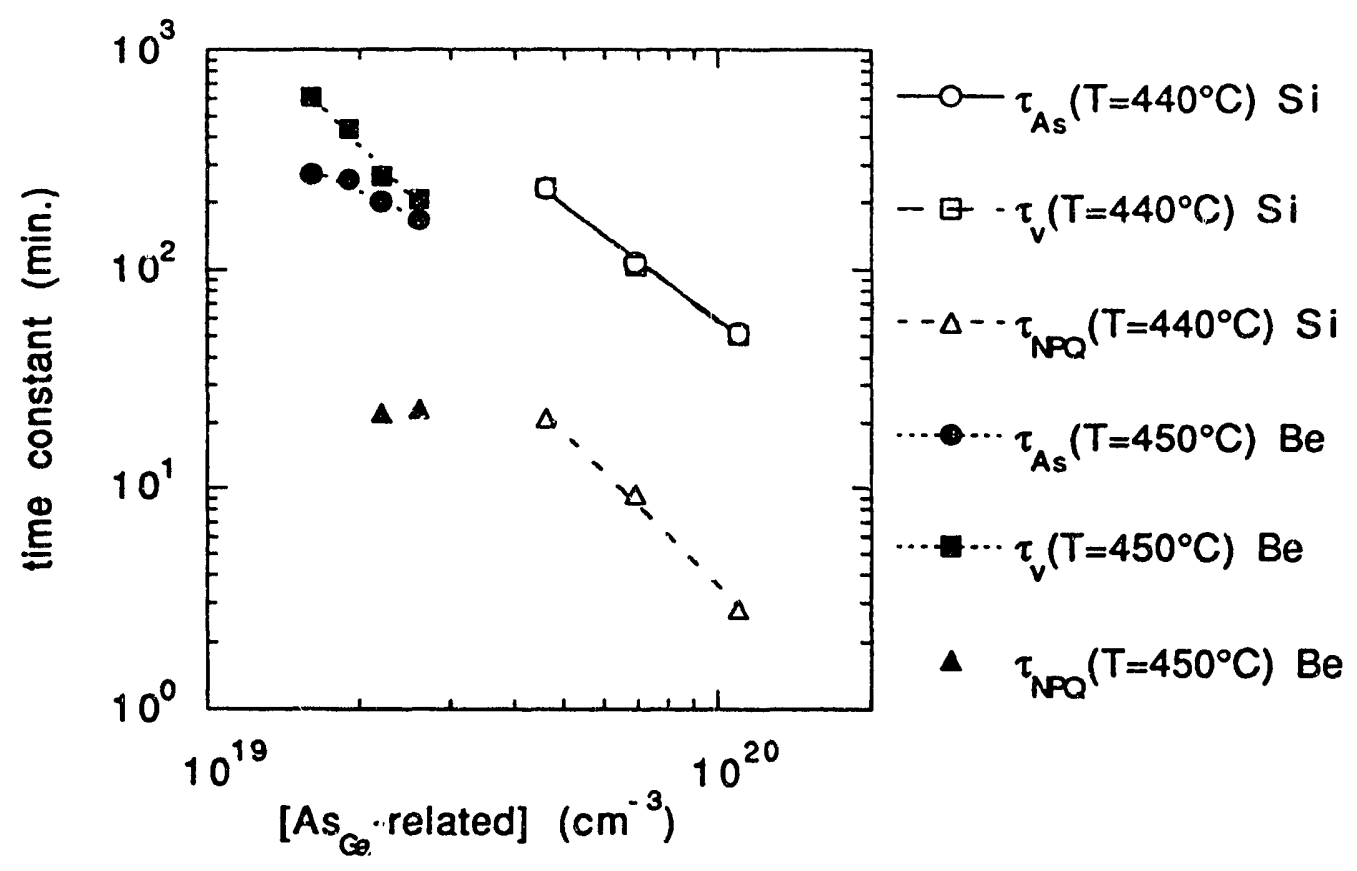

FIG. 3.18 Plot of the the isothermal annealing time constants, $(0) \tau_{A s},(\square) \tau_{v}$, $(\Delta) \tau_{\mathrm{NPO}}$, versus the AsGa-related defect concentration. Filled symbois are the time constants for the Be-doped layers annealed at $450^{\circ} \mathrm{C}$. Open symbols are the time constents for the Si-doped layers annealed at $440^{\circ} \mathrm{C}$. The slope of the log-log plot indicates that all the annealing rates depend approximately on the square of the AsGa-related defect concentration.

range, the consentration dependence of the annealing rate is clearly evident:

$$
\tau^{-1} \propto\left[A s_{G a}\right]^{\alpha}
$$

wher $\alpha=2.0 \pm 0.3$.

The dependence of $\tau_{A s}$ on the concentration of AsGa-related defects can be resolved within the framework of the diffusion model. Annealing of the defects progresses faster with increased defect concentration. To understand the concentration dependence of the time constant, consider eq. $3.8, \tau^{-1}=\lambda^{2} D$. The constant $\lambda$ is 
determined by solving the boundary conditions which give the transcendental equation, $\operatorname{Tan}\left[\lambda\left(R-r_{0}\right)\right]=\lambda R$. Using the series expansion of $\operatorname{Tan}(x)=x+x^{3} / 3+\ldots$, an approximate solution for $\lambda$ is then,

$$
\lambda^{2}=\frac{3 r_{0}}{R^{3}}\left[1+\frac{3 r_{0}}{R}+\ldots\right]
$$

Using the first term of Eq. 3.17, since $\frac{r_{0}}{R} \ll 1$, the expression for the time constant becomes

$$
\tau^{-1}=\frac{3 r_{0} D}{R^{3}}
$$

In terms of the precipitate concentration, $\frac{4}{3} \pi R^{3} C_{p}=1$, the time constant is,

$$
\tau^{-1}=4 \pi C_{p} r_{0} D
$$

It was recently brought to my attention (Gösele 1992) that this is the same expression for $\tau$ as put forth by Smoluchowski (1916) nearly 70 years ago for diffusion limited "coaguiation."

To obtain the concentration dependence of $\tau$ we must postulate on the relationship between the AsGa-related defect concentration and both the concentration of $\mathrm{V}_{\mathrm{Ga}}$ and precipitate nuclei. In the first case we propose that the $V_{G a}$ concentration is proportional to the AsGa-related defect concentration. The justification for this is that as the growth temperature is lowered, more excess As is 
incorporated in the layers as point defects. Since the temperature is low and the defect concentrations are far above equilibrium values no equilibrium is being established between the defects either. Therefore an increased concentration of excess As results in a proportionally higher concentration of both $V_{G a}$ and $A s G a$. This would be analogous to the process of defect introduction in irradiation damaged material. The greater the total incident flux, the more damage, hence the more defects of all kinds are produced. However, instead of irradiating for longer times to create more damage, for LTMBE layers, the growth temperature is lowered increasing the amount of excess As. This implies that the [excess As] $\propto\left[A s_{G a}\right] \propto\left[V_{G a}\right]$. Since the diffusivity depends on the number of available $V_{G a}$ sites to move into, the diffusivity is also proportional to $A s G_{a}$ concentration, $D \propto\left[V_{G a} \propto\left[A s_{G a}\right]\right.$.

We will also argue that the concentration of precipitate nuclei is proportional to the AsGa concentration. A stable precipitate nucleus which can grow must have a critical radius and therefore contain a specific number of As atoms. As the concentration of AsGa-related defects increases, the number of available As atoms from which to form stable nuclei also increases. Therefore $C_{p} \propto$ $\left[A s G_{a}\right]$. Thus, the model predicts that $\tau_{A s^{-1}} \propto\left[A s_{G a}\right]^{2}$. This is in good agreement with the experimentally determined value of $\alpha=2.0 \pm 0.3$.

Note, both $D$ and $C_{p}$ are proportional to the initial concentration of AsGa-related defects. It is important to recognize that this is not the same as $D$ and $C_{p}$ being proportional the instantaneous concentration of AsGa. If this were the case then D could not be separated out of the gradient term of the diffusion 
equation, making it non-linear in spherical coordinates. $C_{p}$ also is proportional to the initial concentration of precipitate nuclei. Once formed, the precipitate concentration should not change drastically. For higher annealing temperatures around $800{ }^{\circ} \mathrm{C}$ this is not true. Arsenic can be released from the precipitates so that some will grow quite large and the smaller precipitates will disappear.

Concentration of $V_{G_{a}}$ and the entropy of migration

Now we will try to answer the question of what concentrations of $\mathrm{VGa}_{\mathrm{Ga}}$ are necessary to promote the observed diffusion. The time constant pre-exponential, $\tau_{\text {As }}^{0}$, can be related to concentration of $\mathrm{V}_{\mathrm{Ga}}$ through the diffusivity. Substituting Eq. 3.5 into Eq. 3.4 into Eq. 3.19 and doing some simple algebraic rearrangement, the following expression is obtained for the concentration of $\mathrm{V}_{\mathrm{Ga}}$ in terms of the annealing pre-exponential,

$$
\left[V_{G a}\right]=\frac{[\text { Ga-sites }]}{\tau_{A s}^{0} 4 \pi C_{p} r_{0} \frac{1}{6} a_{0}^{2} v_{0} \exp \left(\frac{\Delta S_{m}}{k}\right)} .
$$

The experimentally determined quantities such as $\tau_{A s}^{0}, C_{p}$ and $r_{0}$ have been measured or limits can be placed on their values. Other quantities such as $v_{0}, a_{0}$ and [Ga-sites] are well known properties of GaAs. However, theoretical or experimental studies of $\Delta S_{m}$ in III-V semiconductors are few and the values widely scattered. For example, in alkali halides, $\Delta S_{m} / k \sim 1-3 k$ (Varotsos and Alexopoulous 1977). In Si and Ge $\Delta S_{m} / k \sim 4 k$ has been estimated (VanVechten 1975). In an elegant study of heterojunction intermixing (Rouviere, 
et al. 1992) the sum of the formation and migration entropiestd has been estimated to be $\Delta S_{m}+\Delta S_{f} \sim 33 \mathrm{k}$. In another review paper (Tan, et al. 1991) of point defect diffusion the migration and formation entropies are not mentioned, but hidden in the pre-exponential term corresponding to $\Delta S_{m}+\Delta S_{f} \sim 40 \mathrm{k}$. Other theoretical work (Laks, et al. 1991) has placed limits on the entropy of formation of $\Delta S_{f} \leq 10 k$. If we choose the maximum theoretically allowed $\Delta S_{f}=10 \mathrm{k}$, then $\Delta S_{m}$ $\sim$ 20-30 k. Such a wide rage of migration entropies gives a latitude of 13 orders of magnitude in determining the $V_{G a}$ concentration! This does not make for a convincing argument.

Instead I will try a different approach. Given that we know that $\sim 1019 \mathrm{~cm}^{-3} \mathrm{~V}_{\mathrm{Ga}}$ exist from positron annihilation experiments, what value of $\Delta S_{m}$ will yield this concentration from the annealing transient? Rearranging Eq. 3.20 to obtain an expression for $\Delta S_{m}$ in terms of $\tau_{\text {As }}^{\circ}$ and the other experimentally determined quantities,

$$
\Delta S_{m}=-\ln \left[\frac{2 \pi a_{0}^{2} v_{o}}{3[\text { Ga-sites }]} \tau_{A s}^{0} C_{p} r_{0}\left[V_{G a}\right]\right] .
$$

Now the problem lies in estimating $C_{p}$ and $r_{0}$. Unfortunately, in our layers grown at $300^{\circ} \mathrm{C}$ and annealed at $\sim 450{ }^{\circ} \mathrm{C}$, the precipitates are too small to be observed by TEM. The concentration of precipitates necessary to account for the initial concentration of AsGa can be estimated. The TEM detection limit for precipitates is a diameter of approximately $15 \AA$ (Liliental-Weber, et al. 1991). The

$t$ In the paper the formation and migration entropy terms are lumped together and called the entropy of formation. 
microstructure of precipitates less than $30 \AA$ in diameter is a "pseudocubic" phase of arsenic coherent with the GaAs lattice (Liliental-Weber, et al. 1991). The number of excess As atoms per precipitate, $N_{p}^{A s}$ is,

$$
N_{p}^{A s}=\left(\frac{4}{3} \pi r_{0}^{3}\right)[\text { Ga-sites }]
$$

taking the same lattice constant as GaAs for the precipitate. For the TEM detection limit, $r_{0}=8110^{-8} \mathrm{~cm}$, each precipitate contains approximately 50 extra As atoms. To account for an initial concentration of $\sim 311019 \mathrm{~cm}^{-3}$ AsGa-related defects the concentration of precipitates would be $C_{p} \sim 511017 \mathrm{~cm}^{-3}$. Using these values for $r_{0}$ and $C_{p}$ the average value of $\Delta S_{m}=10.8 \mathrm{k}$. The uncertainties of the estimated quantities are $r_{0}$ (factor of 3$), C_{p}$ (factor of 5) and [ $\mathrm{VGa}_{\mathrm{Ga}}$ (factor of 4) to yield $\Delta S_{m}=10.8 \pm 4 \mathrm{k}$.

To decide if this is a reasonable value of $\Delta S_{m}$ we must consider what the entropy of migration for an AsGa means. It is essentially the change in the number of available states of the system due to the AsGa hopping into the vacancy. The states of the system are comprised mainly of the phonons associated with the AsGa atom. The entropy of migration relates to the change in the population of phonons locally around the atom. By hopping, the $V_{G a}$ breaks the local symmetry of the crystal allowing more phonons modes than the available Bloch modes. The entropy of migration is not the entropy of mixing the positions of all the atoms in the crystal. The manifestation of the entropy of migration is the temperature dependent lowering of the energy of migration, i.e., 
$\Delta S_{m}=\frac{\partial E_{m}}{\partial T}$. The entropy of similar processes in GaAs which depend on the presence of phonons is revealed by a comparison of the temperature dependence of the energy barriers.

One fundamental property of semiconductors is the bandgap energy. It is coupled to phonons via the deformation potential. The temperature dependence of the bandgap for GaAs between the temperatures of $500-1000 \mathrm{~K}$ is $5.3110^{-4} \mathrm{eV} \mathrm{K}^{-1}$ (Panish and Casey 1969). This corresponds to an entropy of $6.2 \mathrm{k}$. Another fundamental process is the formation of the crystal. Calorimic measurements of the entropy of fusion in GaAs yield a value of $\Delta S_{\text {fusion }}=16.6 \mathrm{cal}$ mole-1 K-1 $^{-1} 8.3 \mathrm{k}$ (Lichter and Sommelet 1969). This should be a good upper limit to the entropy of any single process in GaAs since the maximum disorder created in a solid should be by melting. Thus our value of $10.8 \pm 4 k$ lies within this range of intuitively accessible entropies.

\section{Sinks for $V_{G a}$}

The pre-exponential of the time constant for the annealing of $V_{G a}$ is significantly larger than for the $A s G a$ by typically 3 to 5 orders of magnitude. This indicates fewer sinks exist for the $V_{G a}$ than for the AsGa. The expression for the diffusivity of the $V_{G a}$ is the same as Eq. 3.4 the antisites but the jump frequency is different,

$$
\Gamma_{j}^{v}=v_{0} \exp \left(\frac{\Delta S_{m}^{v}}{k}\right) \exp \left(\frac{-\Delta H_{m}^{v}}{k T}\right) .
$$

Every neighboring site is available for the $V_{G a}$ to move as long as it is not already occupied by a $V_{G a}$. The $V_{G a}$ hops from site to site until 
it is annihilated. The average number of jumps, $N_{j}$, the $V_{G a}$ makes before it is lost is related to the time constant pre-exponential by,

$$
\left(\begin{array}{ll}
\tau_{0}^{v} & { }^{-1}
\end{array}\right)^{-\frac{v_{0}}{N_{j}}} \exp \left(\frac{\Delta S_{m}^{v}}{k}\right) \text {. }
$$

Since diffusion is a random walk process the average root-meansquare (RMS) distance traveled by a vacancy will be,

$$
\left\langle r^{2}\right\rangle^{\frac{1}{2}}=\left(N_{j} \frac{a_{o}^{2}}{2}\right)^{\frac{1}{2}} .
$$

If a specific crystallographic direction is chosen ( $z$ for instance), then the RMS distance along that direction is

$$
\left\langle z^{2}\right\rangle^{\frac{1}{2}}=\left(N_{j} \frac{a_{0}^{2}}{6}\right)^{\frac{1}{2}} .
$$

Using the same value of $\Delta S_{m}=10.8 \mathrm{k}$ as determined for the AsGa diffusion, the average number of jumps and the root-mean-squared distance traveled by the vacancy are tabulated in Table 3.5 .

With the exception of the $[\mathrm{Be}]=10^{18} \mathrm{~cm}^{-3}$ and $[\mathrm{Be}]=10^{19} \mathrm{~cm}^{-3}$ samples, the root-mean-square distance along the z-direction is on the order of the thickness of the wafer which is $0.5 \mathrm{~mm}$. The diffusion in the two highly Be-doped could be influenced by the presence of the conducting layer at the interface. Without claiming 
Tabie 3.5 Values of the pre-exponential, $\tau_{0}^{V}$, for the annealing of $V_{G a}$ and the calculated values for the average number of hops, $\mathrm{N}_{\mathrm{j}}$, and the root-meansquare distance $\left\langle z^{2}\right\rangle^{\frac{1}{2}}$.

\begin{tabular}{cccc}
\hline $\begin{array}{c}\text { Sample } \\
\text { Doping } \\
\left(\mathrm{cm}^{-3}\right)\end{array}$ & $\tau_{0}^{v}(\mathrm{~min})$. & $N_{j}$ & $\left\langle\mathrm{z}^{2}\right\rangle^{\frac{1}{2}}(\mathrm{~cm})$ \\
\hline$[\mathrm{Be}]=10^{16}$ & $1.5 \times 10^{-7}$ & $3.5 \times 10^{12}$ & $4.3 \times 10^{-2}$ \\
{$[\mathrm{Be}]=10^{17}$} & $1.5 \times 10^{-7}$ & $4.0 \times 10^{12}$ & $4.6 \times 10^{-2}$ \\
{$[\mathrm{Be}]=10^{18}$} & 65 & $1.5 \times 10^{21}$ & 900 \\
{$[\mathrm{Be}]=10^{19}$} & 0.14 & $3.3 \times 10^{118}$ & 42 \\
undoped & $1.6 \times 10^{-9}$ & $3.7 \times 10^{10}$ & $4.4 \times 10^{-3}$ \\
{$[\mathrm{Si}]=10^{17}$} & $1.5 \times 10^{-10}$ & $2.8 \times 10^{-9}$ & $1.2 \times 10^{-3}$ \\
{$[\mathrm{Si}]=10^{19}$} & $1.0 \times 10^{-7}$ & $2.4 \times 10^{12}$ & $3.6 \times 10^{-2}$ \\
\hline \hline
\end{tabular}

to have found a new means to measure the wafer thickness (calipers will still suffice) this suggests that the wafer surfaces are acting as sinks for the $V_{G a}$. It appears that internal sinks such as the layer interface, the arsenic precipitates, or voids are not the dominant gettering sites for vacancies.

One point to address then is the relative stability of $V_{G a}$ in LTMBE layers as compared to electron irradiated GaAs. It has been observed that $\mathrm{VGa}_{\mathrm{G}}$ are not stable at room temperature in electron irradiated GaAs (Jia, et al. 1992). It is important not to confuse the stability of $V_{G a}$ with the stability of interstitials. Irradiation damage of materials generates a high concentration of Frenkel pairs. When the high energy particle knocks out an atom it generates an interstitial leaving behind the vacancy. It is weil known that 
interstitials in semiconductors, whether self-interstitials or transition metals, are highly mobile even at room temperature. $t$ The highly mobile interstitial can easily find the vacancy to recombine. LTMBE GaAs, however, contains very little interstitials as compared to irradiation damaged GaAs. Therefore the $\mathrm{V}_{\mathrm{Ga}}$ should be more stable. An anaolgy can be drawn. In LTMBE GaAs, the more mobile $V_{G a}$ make the less mobile AsGa defects appear less stable, while in electron irradiated material the more mobile interstitials make the $V_{G a}$ appear less stable.

Also, it has been demonstrated that $\mathrm{Zn}$ diffusion is strongly enhanced in LTMBE GaAs (Sin, et al. 1991). Again this result can be understood in terms of $\mathrm{V}_{\mathrm{Ga}}$ facilitated diffusion of $\mathrm{Zn}$ atoms residing on the $\mathrm{Ga}$ sites. Such a process provides a new diffusion channel in addition to the commonly considered substitutional-interstitial diffusion of group II acceptors in GaAs (Gösele and Morehead 1981, Rajeswaran, et al. 1991).

\subsection{Summary and Conclusions}

The annealing of AsGa-related defects in LTMBE GaAs occurs at a temperature significantly below that required to anneal away EL2, the AsGa-related defect in bulk grown crystals. AsGa-related defects in LTMBE GaAs anneal away in two temperature ranges. The first stage relate to the non-photoquenchable AsGa-related defects. It starts at a temperature of $330^{\circ} \mathrm{C}$, just above the growth temperature. The second stage occurs at $450-500^{\circ} \mathrm{C}$ and

$t$ This is why transition metals are such "killers" of semiconductor devices. They can easily diffuse into the active region of devices ruining the electrical properties. 
corresponds to the loss of photoquenchable AsGa-related defects. It is characterized by a rapid decrease in the hopping conductivity as well as a decrease in the intra-center IR absorption at a photo 1 wavelength, $\lambda=1 \mu \mathrm{m}$. These annealing stages are quite similar to the annealing of AsGa-related defects in heavily damaged, irradiated or plastically deformed GaAs. However, it is in contrast to EL2 in bulk GaAs which is stable up to $1100^{\circ} \mathrm{C}$.

We have proposed that the lower temperature annealing is due to defect enhanced diffusion of AsGa-related defects. The presence of a high concentration, $\sim 10^{19} \mathrm{~cm}^{-3}$, of $V_{G a}$ facilitates the diffusion of AsGa-related defects to arsenic precipitates present in the material. Like the AsGa-related defects the concentration of $\mathrm{V}_{\mathrm{Ga}}$ is supersaturated and decreases also. To account for the loss of $\mathrm{V}_{\mathrm{Ga}}$ we have worked out a model using a time dependent diffusivity. The model describes the annealing transients extremely well, using only one time constant parameter for each defect. The model is general and applies to the $V_{G a}$ enhanced diffusion of any atomic species in damaged GaAs materials which coniain a high concentration of $\mathrm{V}_{\mathrm{Ga}}$. An Arrhenius plot of the time constant parameter for each of the defects yields an activation enthalpy for migration of $1.1 \pm 0.3 \mathrm{eV}$ for the NPQ AsGa-related defects, is $1.5 \pm 0.3 \mathrm{eV}$ for the $V_{G a}$ and 2.0 $\pm 0.3 \mathrm{eV}$ for the PQ AsGa-related defects. The difference in migration enthalpy, $0.5 \mathrm{eV}$, between the $\mathrm{V}_{\mathrm{Ga}}$ and $P \cdot Q$ defects represents the difference between an $\mathrm{As}$ atom or a $\mathrm{Ga}$ atom hopping into the vacancy.

The NPQ defects anneal away much more easily with an activation enthalpy nearly half that of the $P Q$ defects. The identity 
of the non-photoquenchable defects is not certain. We have postulated that the non-photoquenchable defects have a neighboring defect within 3 nearest neighbor distances which disturb the metastability. We have argued that strain associated with the neighboring defect or nearby precipitate is capable of lowering the barrier to migration by approximately $1 \mathrm{eV}$. A more exact explanation will require detailed information about the identity of the non-photoquenchable defects.

The rate of annealing is concentration dependent and depends on the square of the concentration of AsGa-related defects. This can be accounted for in the model by assuming that the concentrations of antisites, vacancies and precipitate nuclei are all proportional to the excess As content. Analysis of the pre-exponential of the temperature dependence of the annealing time constants yields a migration entropy on the order of $10 \mathrm{k}_{\mathrm{B}}$ for the $\mathrm{V}_{\mathrm{Ga}}$. It also appears that the sink for the excess $V_{G a}$ is the sample surface.

For the intentionally introduced $n$ - and p-type shallow impurities, $\mathrm{Be}$ and $\mathrm{Si}$, free carriers can be activated after annealing at $800^{\circ} \mathrm{C}$. Typically, $800^{\circ} \mathrm{C}$ is the temperature used to activate ion implanted dopants. The Si, however, can only be partially activated. The fact that the dopants require annealing at $800^{\circ} \mathrm{C}$ to activate while the AsGa-related defects anneal away at $450^{\circ} \mathrm{C}$ leads us to believe that the dopants form defect-impurity complexes. Annealing at higher temperature is required to dissociate the pair. For the $\mathrm{Be}$ we attribute the activation to the break-up of $A s G_{a}-B e$ donoracceptor pairs. 
The origin of the partial activation of the $\mathrm{Si}$ is not clear. It could be due to Si-defect complexes or it could be due to the fact that the $\mathrm{Si}$ has not moved to a substitutional site. Further investigation of the annealed $\mathrm{Si}$ doped material with Raman spectroscopy could clarify this point. Also, it might be possible to activate a larger fraction of the $\mathrm{Si}$ by annealing at higher temperature. Often n-type ion implants are annealed at temperatures $50-100^{\circ} \mathrm{C}$ higher than for p-type implants. It would also be interesting to see if the lack of electrical activation also applies to group VI donors such as Te or Se. In addition it would be useful to study the heavier group IV donor $\mathrm{Sn}$ and attempt RBS channeling experiments to locate the position of the Sn.

On a final note, I would like to emphasize that besides nicely showing the difference in energy between an $\mathrm{As}$ atom and $\mathrm{Ga}$ atom motion on the group III sublattice, this work has technological applications. It can be used as a guideline for determining annealing conditions to obtain a desired concentration of thermally stable photoquenchable AsGa-related defects. By careful annealing at lower temperatures for longer time than is typically done for the preparation of LTMBE buffer layers, non-photoquenchable and other undesirable defects can be eliminated while stabilizing a desired concentration of photoquenchable EL2-like defects with a well defined electronic level at midgap. This type of annealing soak would be beneficial to fast optical switches and terrahertz pulse generators fabricated from LTMBE GaAs. 


\section{References}

Coates, R. and E.W.J. Mitchell. Advan. in Phys. 24, 593 (1975).

Dlubek, G. and R. Kruse. "Positron studies of defects in III-V semiconductor compounds." Phys Stat. Sol. (a) 102, 443 (1987).

Farmer, J.W. and D.C. Look. "Electron-irradiation defects in n-type GaAs." Phys. Rev. B. 21, 3389 (1980).

Gösele, U. (private communication 1992).

Gösele, U. and F. Morehead. J. Appl. Phys. 52, 4617 (1981).

Jia, Y.Q., H.J. von Bardeleben, D. Stievenard and C.J. Delarue. " Electronparamagnetic-resonance observation of gallium vacancy in electronirradiated p-type GaAs." Phys. Rev. B45, 1645 (1992).

Kuznetsov, V.P., M.A. Messerer, E.M. Omelhanovsky and A.J. Polyakov, "The evidence for the Existance of the Hopping Conductivityvia Deep Impurity States: InP:Mn." J.D. Chadi and W.A. Harrisons, ed., $17^{\text {th }}$ International Conference on the Physics of Semiconductors (ICPS) (Springer Verlag, San Francisco, CA, USA, 1984) pp. 643.

Laks, D.B., C.G. Van de Walle, G.F. Neumark and S.T. Pantelides. "Role of native defects in wide band gap semiconductors." Phys. Rev. Lett. 66, 648 (1991).

Lichter, B.D. and P. Sommelet. Trans. Metal. Soc. AIME 245, 1021 (1969).

Liliental-Weber, Z., A. Claverie, J. Washburn, F.W. Smith and A.R. Calawa. "Microstructure of annealed low-temperature-grown GaAs layers." Appl. Phys. A 53, 141 (1991).

Panish, M.B. and H.C. Casey. J. Appl. Phys. 40, 163 (1969).

Rajeswaran, G., K.B. Kahen and D.J. Lawrence. J. Appl. Phys. 69, 1359 (1991).

Rouviere, J.-L., Y. Kim, J. Cunningham, J.A. Rentschler, A. Bourret and A. Ourmazd. "Measuring Properties of Point Defects by Electron Microscopy: The Ga Vacancy in GaAs." Phys. Rev. Lett. 68, 2798 (1992).

Rytova, N.S. and E.V. Soloveva. Sov. Phys. Semicon. 20, 869 (1986).

Shklovskii, B.I. and A.L. Efros, "Electronic Properties of Doped Semiconductors." M. Cardona, Ed., (Springer-Verlag, Berlin, 1984a). 
Shklovskii, B.I. and A.L. Efros, "Electronic Properties of Doped

Semiconductors." M. Cardona, ed. (Springer-Verlag, Berlin, 1984b) pp. 139.

Sin, Y.K., Y. Hwang, T. Zhang and R.M. Kolbas. J. Electron. Mater. 20, 465 (1991).

Smoluchowski, M.v. "Drei Vorträge übar Diffusion, Brownsche Molekularbewegeng und Koagulation von Kolloidteilchen." Physik. Zeitschr. 17, 585 (1916).

Tan, T.Y., U. Gösele and S. Yu. "Point defects, diffusion mechanisms, and superlattice disordering in gallium arsenide-based materials." Crit. Rev. Solid State Mat. Sci. 17, 47 (1991).

VanVechten, J.A. J. Electrochem. Soc. 122, 423 (1975).

Varotsos, P. and K. Alexopoulous. Phys. Rev. B15, 2348 (1977).

Walukiewicz, W., L. Lagowski, L. Jastrzebski, M. Lichtensteiger and H.C. Gatos. "Electron mobility and free-carrier absorption in GaAs: Determination of the compensation ratio." J. Appl. Phys. 50, 899 (1979).

Weber, E.R. and J. Schneider. Physica 116B, 398 (1983).

Wei, L., Y. Tabuki, S. Tanigawa, D.E. Bliss, W. Walukiewicz and E.E. Haller. "Characterization of point defects in GaAs grown at low temperatures by molecular beam epitaxy." (submitted to Appl. Phys. Lett. 1992).

Zhang, S.B. and J.E. Northrup. "Chemical potential depencence of defect formation energies in GaAs - Application to Ga self-diffusion." Phys. Rev. Lett. 67, 2339 (1991). 


\section{Appendix A: $\quad$ Major properties of EL2 in GaAs}

\section{A.1. Introduction}

In this appendix the major properties of the defect in GaAs known as EL2 will be reviewed. EL2 is the main native deep level defect found in GaAs grown by bulk and vapor-phase-epitaxial (VPE) techniques under arsenic-rich conditions. It is of prominent technological importance for its role compensating shallow level impurities. The production of high quality substrates, using EL2 to produce semi-insulating properties is the foundation for an ion implantation fabricated, high speed, integrated circuit (IC) technology. The main scientific interest in EL2 stems from its metastable properties. In this regard, EL2 is closely related to the deep level $D X$ center in $A l_{x} G a_{1-x} A s$ associated with the introduction of donor impurities. This appendix will summarize the role of deep levels in semiconductors in general, present the major properties of EL2, such as electronic levels, photoquenching properties, and properties under pressure, and discuss the proposed models of EL2 and how they account for the metastability.

\section{A.2. Deep level defects}

Deep levels in general are of interest both fundamentally and technologically. Unlike shallow levels, whose excitation behavior is so well predicied by effective mass theory, theorists have yet to provice a general formalism which predicts the physical properties 
of deep centers. Experimental probing of these defects is further characterized by ambiguous results. It is understood that the presence of deep levels, even in minute quantities greatly affects the properties of semiconducting materials.

The most significant effects of deep levels are,

1) the degradation of free carrier lifetimes

2) the compensation of shallow level impurities

3 ) the influence of photo-efficiencies.

Take the case of free carrier lifetimes. Consider two energy levels in a material, one shallow and one deep. If the energy difference between the levels is greater than $0.4 \mathrm{eV}$, then at room temperature it can be shown (Grimmeiss 1977) that the lifetime of excess carriers will be determined by the concentration of the deeper level, even if it is $N_{D} \sim N_{S} \times 10^{-6}$. Thus the concentration of deep levels should be limited well below shallow impurity concentrations to fabricate efficient devices.

A case where deep levels would be desirable is if one were trying to produce semi-insulating (SI) substrates. By compensating shallow impurities, thus eliminating easily ionized free carriers, material having intrinsic-like properties can be produced. Analytically consider the system of three levels,

$N_{A}$ - concentration of shallow acceptors

ND - concentration of shallow donors

NDD - concentration of deep donors.

Without any deep donors the net shallow impurity concentration would have to be below the intrinsic carrier concentration, $\left|N_{D}-N_{A}\right|<n_{i}$, which for GaAs at room temperature is $\sim 2 \times 10^{6} \mathrm{~cm}^{-3}$. 
It is currently impossible for crystal growers to obtain such high purities. The purest solid available is ultra-high purity Ge with a background impurity concentration of $\sim 10^{10} \mathrm{~cm}^{-3}$. With a deep donor present, the limitation on the net acceptor concentration becomes $\left(N_{A}-N_{D}\right)<N_{D D}$. For a typical concentration of EL2, NDD $1016 \mathrm{~cm}^{-3}$. Thus, to the great relief of the crystal grower, the impurity limits have been eased 10 orders of magnitude.

It is a fortuitous phenomenon that a native defect is able to be used as a deep level at midgap. SI material can be created without introducing a deep level impurity, typically the transition metal chromium. Transition metals are notoriously fast diffusers, causing problems associated with migration and precipitation in the active regions of devices. Thus the existence of the native defect EL2 in GaAs is desirable, making it imperative to understand and control the defect concentration as opposed to just eliminating it.

\section{A.3. Properties of EL2}

\subsection{Electronic energy levels and the $\mathrm{CC}$ diagram}

EL2 is a deep double donor with two transition levels in the bandgap. Most of the electronic and optical transitions of EL2 can be summarized in a configurational coordinate (CC) diagram (see Fig.

A.1). A CC diagram shows the sum of the the electronic and elastic energies of a defect versus the configuration of the the defect expressed as some generalized coordinate. In a completely general sense, the configurational coordinate refers to the distortion of the defect. This distortion has many possibilities. It could be the linear displacement of an atom, a breathing-mode motion of atoms 
surrounding the defect or some other distortion of the lattice in the vicinity of the defect. The elastic contribution to the energy is represented as a parabola on the $\mathrm{CC}$ diagram. This assumes Hooks law applies: specifically a constant and symmetric force constant, $F=K Q$, where $F$ is the force, $Q$ is the configurational coordinate and $K$ is the force constant. The notation used in the CC diagram for EL2 is summarized as follows. The equilibrium positions are marked on the $x$-axis. The presence of $a^{*}$ within the brackets denotes the metastable state and the superscript outside the brackets denotes the charge state. For example, $\left(E L 2^{*}\right)^{\circ}$ denotes the neutral metastable state, while $(E L 2)^{+}$denotes the singly ionized normal state. The parabolas are labeled by the charge state of the defect and the electron occupancy, i.e., $(E L 2)++\dot{\theta}_{B}$ identifies the singly ionized normal state with the electron in the conduction band. Such an identification will always give a charge neutral label for each parabola. 


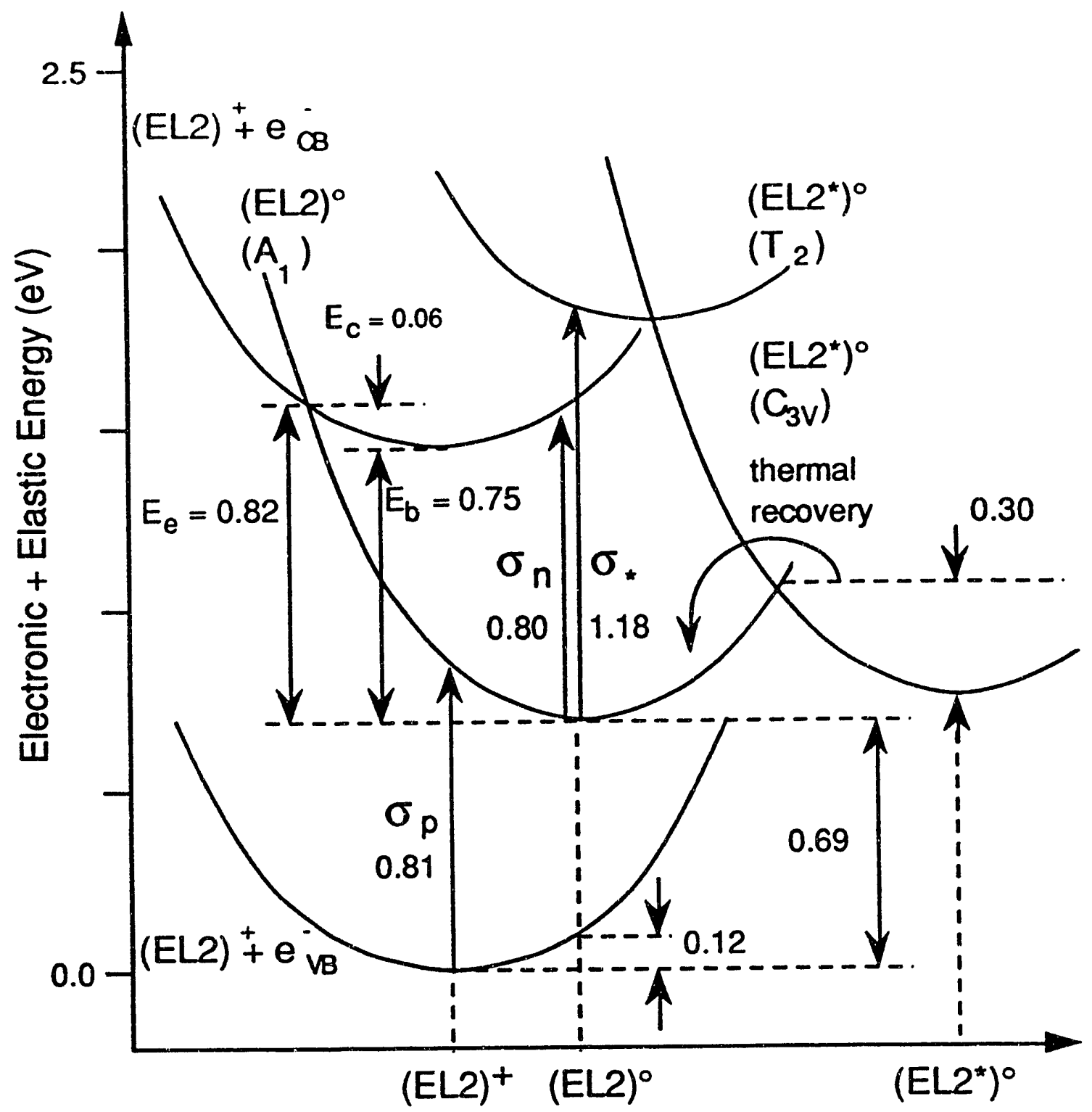

\section{Generalized Configurational Coordinate}

FIG. A.1 Configurational coordinate diagram for neutral and singly ionized EL2 in the normal state and the excited and metastable states, EL2*. The yaxis is the sum of the electronic state energy plus the elastic energy associated with the deformation of EL2 in units of $\mathrm{eV}$. Parabolas represent a simple Hook's law approximation of the elastic energy. Ground state configurations of each state are indicated on the $x$-axis. The generalized coordinate is believed to be a (111) displacement of the AsGa atom. Experimentally determined energies between various states are indicated within the diagram. Values are discussed and referenced in the text of appendix $A$. 


\subsection{Energy levels determined by Hall effect and DLTS}

The electron binding energy of the first ionization level of EL2 is $E_{b}=0.75 \mathrm{eV}$ and has been determined from the temperature dependent Hall effect measurements (Kitagawara, et al. 1986). In the $\mathrm{CC}$ diagram $E_{\mathrm{b}}$ corresponds to the energy difference between the minima of the $(E L 2)^{\circ}$ and $(E L 2)^{+}+\theta_{C B}^{-}$parabola. When the Fermi level is pinned to this level at midgap the intrinsic-like SI properties of GaAs are realized. Deep level transient spectroscopy (DLTS) measurements yield electron emission and capture barriers to and from the conduction band of $E_{e}=0.82 \mathrm{eV}$ and $E_{c}=0.06 \mathrm{eV}$ respectively (Lang and Logan 1975).

\subsection{Energy levels determined by optical means}

An excellent review of the optical properties of EL2 has been published by Kaminska (1988). The cross sections for the optical transitions of an electron to the conduction and valence bands, $\sigma_{n}$ and $\sigma_{p}$, were determined by photocapacitive techniques and were the first IR absorption measurements made of EL2 (Bois, et al. 1979). The characteristic IR absorption of EL2 (Martin 1981) in the range $1.0-1.3 \mathrm{eV}$ (labeled $\sigma_{*}$ in Fig. A.1) was discovered to be due to an intracenter transition by comparison of photoionization and IR absorption spectra (Kaminska, et al. 1983). The excess IR absorption as compared to the photoionization current is regarded as an $A_{1}$ to $T_{2}$ intracenter transition. The peak at $1.18 \mathrm{eV}$ corresponds to the "vertical" (no configurational change between initial and final states) transition $(E L 2)^{\circ}$ to $\left(E L 2^{*}\right)^{\circ}$.

\subsection{Zero-phonon-line}


Below the intracenter transition at an energy of $1.04 \mathrm{eV}$, is the zero-phonon-line ( $Z P L)$ with multiphonon replicas spaced above it at intervals of $11 \mathrm{meV}$ (Kaminska 1988). Measurements of the intensity of the ZPL and of the intracenter absorption show a direct proportionality to the EL2 concentration determined by DLTS measurements (Skowronski, et al. 1986). However, if the absorption at 1.18 is the vertical intracenter transition of the ZPL, the FranckCondon shift dFC is 1.18-1.04 $=0.14 \mathrm{eV}$. Also, if the $11 \mathrm{meV}$ replicas are due to the lattice relaxation phonon, then the Huang-Rhys facor $S H R=d F C / \hbar \omega$ is approximately 13 . Given these two suppositions, theory predicts a relative intensity for the ZPL of $e^{-13} \sim 10^{-6}$ which would make it unobservable. Either the lattice relaxation phonon energy is incorrect or the ZPL and intracenter absorption are not due to the same state. Further complicating the picture is the observation that under hydrostatic pressure, the ZPL is observed to move in the opposite direction to the intracenter absorption, riding up over the onset of the broad peak (Baj and Dieszer 1989). This ambiguity leaves these features open to various interperetations (Baraff 1990).

However, analysis of the splitting of the ZPL under uniaxial stress clearly indicates the center has full tetrahedral $\left(T_{d}\right)$ symmetry (Kaminska, et al. 1985). Recent piezo-spectroscopic measurements of the narrow luminescence lines under uniaxial stress confirm the $T_{d}$ symmetry (Nissen, et al. 1991).

\subsection{Magnetic properties}

Electron paramagnetic resonance (EPR) has been the key experiment linking EL2 to the AsGa defect (Wagner, et al. 1980, 
Weber, et al. 1982). For the singly ionized $\mathrm{AsGa}^{+}$defect, the hyperfine interaction between the unpaired spin of the remaining electron and the $I=3 / 2$ arsenic nucleus results in the characteristic "quadruplet" spectrum. The direct correlation between the quadruplet intensity and the EL2 concentration has lead to the identification of AsGa as the main component of the EL2 defect.

The photo-response of the EPR signal has also coincided with the electronic levels of EL2 in the gap. The second ionization level of EL2 has been identified with this technique and lies at $0.52 \mathrm{eV}$ (Baeumler, et al. 1985). Specially prepared p-type samples which also contain EL2 have made it possible to study the second ionization level by DLTS (Bliss, et al. 1990, Lagowski, et al. 1985).

\subsection{Metastability}

As an intellectual subject, the metastable properties of EL2 are perhaps the most interesting aspect of the defect. Theorists can ponder the mechanisms which produce metastable defects while for the experimentalist, the dynamic properties of a metastable defect open up many avenues of investigation as compared to a static defect. Although many mechanisms leading to metastabiiity have been previously proposed, it is generally accepted that metastability is the result of atomic motion in the vicinity of the defect. This motion is referred to as a large laitice-relaxation even if it does not involve atoms of the lattice. If an energetic barrier exists for the atom(s) to return to the equilibrium position(s) then the defect is metastable. A mechanical analog to metastability, the overcenter hinge with springs, is illustrated is Fig. 1.3. 
The metastable nature of EL2 is clearly seen by the photobleaching properties of the the IR absorption. Illumination by light in the range of the intracenter transition, 1.0-1.3 eV, at low temperatures eliminates the entire sub-bandgap IR absorption due to EL2 (Martin 1981, Vincent, et al. 1982). Thus the $T_{2}$ excited state of EL2 is thought to be the intermediate state which undergoes the lattice relaxation into the metastable state $\left(E L 2^{*}\right)^{\circ}$. Photocapacitive measurements indicate no change in charge in converting to the metastable state. Therefore the metastable state is also neutral.

The normal state of EL2 can be recovered by warming the sample. For SI GaAs the recovery occurs for temperatures above approximately $130 \mathrm{~K}$. The recovery temperature is reduced by the presence of free electrons (Mitonneau and Micea 1979, Vincent, et al. 1982). For n-type material the recovery temperature can be as low as $45 \mathrm{~K}$. Analysis of the thermally activated rate of recovery yield a barrier of $0.3 \mathrm{eV}$ (Vincent, et al. 1982) or $0.36 \mathrm{eV}$ (Trautman, et al. 1988). Analysis of the recovery under uniaxial stress indicate that the metastable state is trigonally distorted $\left(C_{3 v}\right)$ from the tetrahedral symmetry of the ground state (Trautman and Baranowski 1992).

\subsection{Microscopic models of EL2 structure}

A great deal is known about the optical and electronic properties of EL2 and can be summarized quite concisely in terms of the phenomenological CC diagram. However, knowledge of the precise structure of the EL2 defect, outside the fact that it must include an AsGa, is quite vague in comparison. None the less, models 
of EL2 which have gained acceptance fall into two categories; one involving the isolated $A s G a$ or the other involving an $A s G a$ plus another defect, generally considered to be an arsenic interstitial, As $_{\mathrm{i}}$. Distinguishing between the twso groups of models experimentally has proven to be díficult. Consequentially, discussions of the microscopic structure of EL2 can sometimes become rather rhetorical.

Any convincing microscopic model of EL2 must display the general characteristics of the defect and account for the metastability. To be more specific, it must be a deep double donor with $T_{d}$ symmetry and involve a trigonal distortion to the metastable state while remaining neutral. Also, the defect must be thermally stable up to a temperature of $1000{ }^{\circ} \mathrm{C}$ when not in the presence of $\mathrm{V}_{\mathrm{Ga}}$.

In calculating the energy levels of the theoretical model, generally local density theory or empirical tight binding approaches are used. The relative accuracy and strength of each theoretical technique has been discussed by Lannoo (1986). However, calculated theoretical energy levels which match experimental values should not be considered a priority in determining the validity of a model. What should be emphasized are the defect charge state and determination of whether the level is shallow or deep.

Historically, metastability in semiconductors has been associated with defect complexes such as interstitial iron-acceptor pairs and the interstitial carbon-substitutional carbon pair in silicon (Watkins 1988). It was believed that the intricacy of a defect complex was required to achieve more than one configuration 
yielding a local minimum in energy. Thus it was proposed that an $A s G a$ plus an $A_{s}$ at two interatomic distances from the $A_{s G a}$ formed the EL2 defect (Von Bardeleben, et al. 1985, Von Bardeleben, et al. 1986). This model is supported by the results of optically detected EPR and ENDOR measurements (Meyer, et al. 1986). These measurements suggest the presence of another distant defect with the antisite.

The major drawback of this model is that it predicts a shallow donor level associated with EL2. The extra electron on the interstitial should be easily ionized. No shallow level has yet been discovered for EL2 casting doubt about the accuracy of the AsGa $+\mathrm{As}_{\mathrm{i}}$ model. Also the Asi should be to diffuse away from the center very easily in contradiction to the observed thermal stability of EL2.

Other models for EL2 have been proposed in the category of an AsGa defect complex with variations on what the other defect should be. Another theme involves a $V_{G a}$ or the $V_{G a} V_{A s}$ pair in addition to the AsGa as proposed by Zou (1982) and followed up by Wager and Van Vechten (1987). However, these models do not describe all the main features of EL2. Divacancies are known to readily anneal away at $450^{\circ} \mathrm{C}$ so that the model does not show thermal stability of EL2. Also, the metastability is modeled to be an internal excitation of the $V_{G a}^{-}$in the complex $V_{A s}^{+} V_{G a}^{-} A_{G a}^{+}$. However this cannot be the case since the $\mathrm{As}_{\mathrm{Ga}}^{+}$would be in the paramagnetic state. It is observed experimentally that the photoquenching occurs in the neutral, diamagnetic state $\mathrm{As}_{\mathrm{Ga}}^{\circ}$. Also the presence of a nearby 
vacancy cannot explain the ENDOR data of the AsGa since the vacancy has no nucleus to interact with the electron spin.

The most promising model of EL2 thus far appears to be the isolated AsGa. It exhibits most of the main features of EL2. The electronic levels of the AsGa can be understood intuitively in a molecular fashion without resorting to the output of lengthy computer code. Arenic and gallium atoms are similar, only being separated by $\mathrm{Ge}$ in the periodic table. Hence they are very similar in size and differ in valence by 2 electrons. When an As atom replaces a $\mathrm{Ga}$ atom, the same covalent $\mathrm{sp}^{3}$ bonds are formed. Thus the two extra electrons result in the center being a double donor. However, As is more electronegative than $\mathrm{Ga}$ so that the antibonding states of the AsGa have a lower energy than those of GaGa. Antibonding states of $A_{1}$ symmetry are lowered out of the conduction band while the $T_{2}$ state remains resonant in the conduction band. The two extra electrons occupy the $A_{1}$ state resulting in the diamagnetic neutral ground state of EL2. Removal of one electron results a paramagnetic state tha: is EPR active.

Theoretical computer calculations have tried to estimate the energy positions of the $\mathrm{EL}_{2} \mathrm{O} /+$ and $\mathrm{EL}_{2}^{+/++}$transitions. and have ranged from midgap to the conduction band edge. However, all the calculated $A_{1}$ to $T_{2}$ transitions have been of the order of $1.0 \pm 0.1 \mathrm{eV}$. This value is very close to the intracenter excitation energy of EL2. One might claim this is only a coincidence. However, theoretical calculations are generally quite good at predicting the rolative energies of excited states while the absolute energy of a ground state is less accurate. The higher accuracy of calculating relative 
energies is very similar to the case of using effective mass theory to calculate the excited states of shallow hydrogenic impurities.

Analysis of the splitting of the ZPL under uniaxial stress is consistent with an $A_{1}$ to $T_{2}$ transition (Kaminska, et al. 1985) strengthening the belief that an isolated ASGa is responsible for EL2. Further analysis of the stress splitting demonstrated that only $T_{d}$ symmetry, and no other, could explain the observed results (Baraff 1989).

Perhaps the most important aspect supporting this identification of EL2 came with the realization that an isolated ASGa could demonstrate metastability. Chadi and Chang (1988) proposed a metastable interstitial configuration for the excited state of an AsGa. They based their claim on ab initio pseudopotential totalenergy calculations and allowed the As atom to move along the [111] direction. The two stable positions of the structure are shown in Fig. A.2. The first structure, with $T_{d}$ symmetry, is the stable state with the antisite forming $s^{3}$ bonds to the four nearest neighbor arsenic atoms. The second structure, with $\mathrm{C}_{3 v}$ symmetry, is the metastable state. The AsGa atom has broken one bond to a neighboring As atom and moved through the plane of the other three As atoms to a threefold coordinated interstitial position. The trigonal distortion created by the motion of the As atom is consistent with the uniaxial stress measurements of the metastable state by Trautman et. al. (1988). The bonding in the metastable state is comprised mainly of an $\mathrm{sp}^{2}$ hybridization. The $s p^{2}$ bonding is characteristic of the puckered-plane structure of group $V$ elements which include arsenic. Other theoretical calculations have 

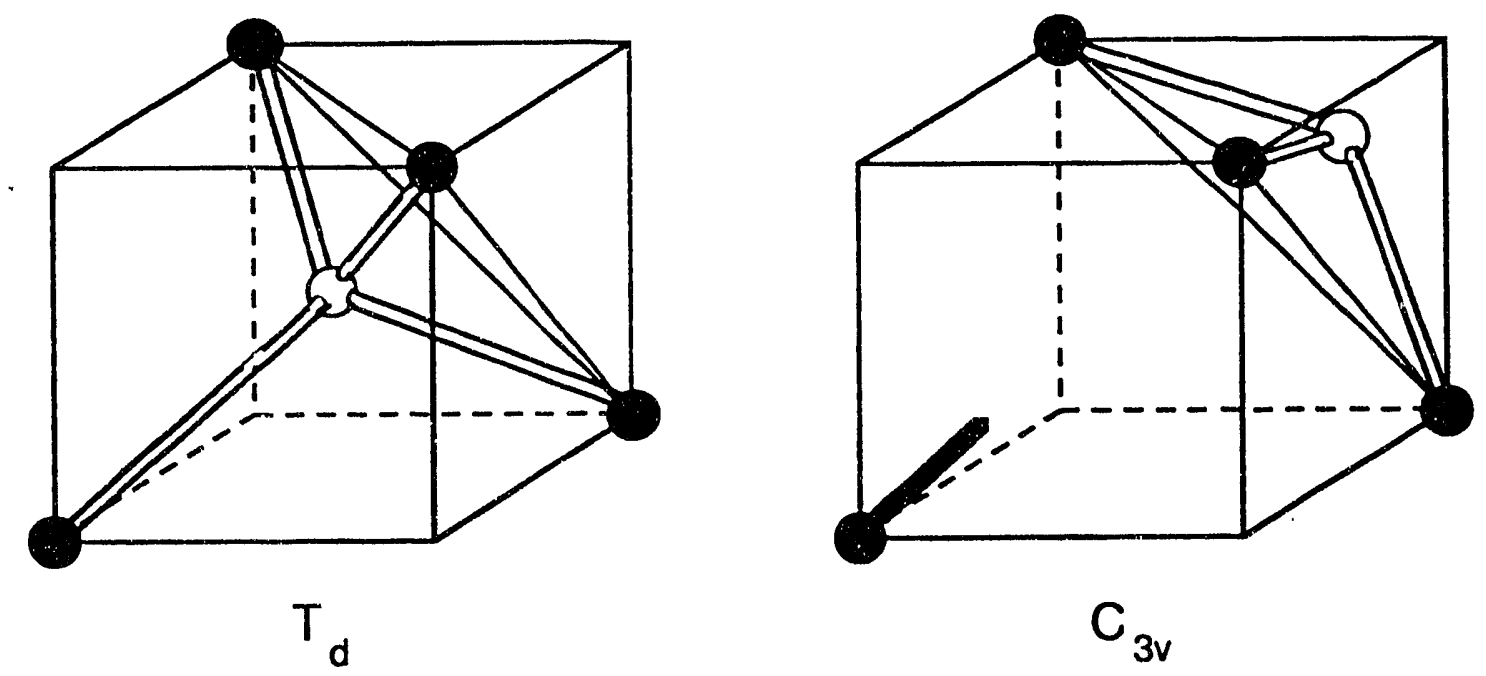

FIG. A.2 Model of EL2 metastability proposed by (Chadi and Chang 1988): The open circle represents the AsGa defect in the stable fourfold coordinated $T_{d}$ state and the metastable threefold coordinated $\mathrm{C}_{3 \mathrm{v}}$ state.

confirmed the metastability of this structure (Dabrowski and Scheffler 1989). Models of the isolated AsGa explain most of the experimentally observed properties of the normal and excited states, including the metastability of the EL2 defect center in GaAs.

\section{References}

Baeumler, M., U. Kaufmann and J. Windscheif. "Photoresponse of the As $\mathrm{Ga}$ antisite defect in as-grown GaAs." App. Phys. Lett. 46, 781 (1985).

Baj, M. and P. Dreszer. "EL2 Intracenter Absorption under Hydrostatic Pressure." Mat. Science Forum 38-41, 101 (1989).

Baraff, G.A. Phys. Rev. B 40, 1030 (1989).

Baraff, G.A. "Stress splitting of the EL2 zero-phonon line: Need for reinterpretation fo the main optical transition." Phys. Rev. B 41, 9850 (1990).

Bliss, D.E., W. Walukiewicz, D.D. Nolte and E.E. Haller. "Absolute pressure dependence of the second ionization level of EL2 in GaAs." Appl. Phys. Lett. 56, 1143 (1990). 
Bois, D., C. A., G. Vincent and A. Novailhat. Inst. Phys. Conf. Ser. 43, 295 (1979).

Chadi, D.J. and K.J. Chang. "Metastability of the isolated arsenic-antisite defect in GaAs." Phys. Rev. Lett. 60, 2187 (1988).

Dabrowski, J. and M. Scheffler. "Isolated arsenic-antisite defect in GaAs and the properties of EL2." Phys. Rev. B 40, 10391 (1989).

Grimmeiss, H.G. Ann. Rev. of Mat. Sci. 341 (1977).

Kaminska, M. “Optical properties of EL2." Rev. Phys. Appl. 23, 793 (1988).

Kaminska, M., M. Skowronski and W. Kuszko. "Identificatiion of the 0.82-eV electron trap, EL2, in GaAs, as an isolated antisite arsenic defect." Phys. Rev. Lett. 55, 2204 (1985).

Kaminska, M., M. Skowronski, J. Lagowski, J.M. Parsey and H.C. Gatos. Appl. Phys. Lett. 43, 302 (1983).

Kitagawara, Y., N. Noto, T. Takahashi and T. Takenaka, "Effects of annealing on resistivities of SI and n-type semi-conducting In-alloyed LEC GaAs," ed. (Shiva publishing, Ohmsha, 1986) pp. 273.

Lagowski, J., D.G. Lin, T.-P. Chen, M. Skowronski and H.C. Gatos. "Native hole trap in bulk GaAs and its association with the double-charge state of the arsenic antisite defect." Appl. Phys. Lett. 47, 929 (1985).

Lang, D.V. and R.A. Logan. "A study of deep levels in GaAs by capacitance spectroscopy." J. Electonic Mat. 4, 1053 (1975).

Lannoo, M., M. Stoneham, ed. (Adam Hilger, Bristol, 1986) vol. 2,.

Martin, G.M. "Optical assessment of the main electron trap in bulk semiinsulating GaAs." Appl. Phys. Lett. 39, 747 (1981).

Meyer, B.K., D.M. Hormann and J.-M. Spaeth, v. Bardeleben, ed. (Trans. Tech. Publ., 1986) vol. 10-12, pp. 311.

Mitonneau, A. and A. Micea. Phys. Rev. B 43, 2070 (1979).

Nissen, M.K., A. Villemaire and M.L.W. Thewalt. Phys. Rev. Lett. 67, 112 (1991).

Skowronski, M., J. Lagowski and H.C. Gatos. J. Appl. Phys. 59, 2451 (1986).

Trautman, P. and J.-M. Baranowski. "Evidence for trigonal symmetry of the metastable state of the EL2 defect in GaAs." Phys. Rev. Lett. 69, 664 (1992). 
Trautman, P., M. Kaminska and J.-M. Baranowski. Cryst. Res. Technol. 23, 413 (1988).

Vincent, G., D. Bois and C. A. "Photoelectric memory effect in GaAs." J. Appl. Phys. 53, 3643 (1982).

Von Bardeleben, H.J., D. Stievenard, J.C. Bourgoin and A. Huber. Appl. Phys. Lett. 47, 970 (1985).

Von Bardeleben, H.J., D. Stievenard, D. Deresme, A. Huber and J.C. Bourgoin. Phys. Rev. B 34, 7192 (1986).

Wager, J.F. and J.A. Van Vechten. Phys. Rev. B 35, 2330 (1987).

Wagner, R.J., J.J. Krebs, G.H. Strauss and A.M. White. Solid State Commun. 36, 15 (1980).

Watkins, G.D., "Defect metastability and bistability." International Conference on Defects in Semiconductors (Budapest, 1988).

Weber, E.R., H. Ennen, U. Kaufmann, J. Windscheif, J. Schneider and T. Wosinski. "Identification of $\mathrm{As}_{\mathrm{Ga}}$ antisites in plastically deformed GaAs." J. Appl. Phys. 53, 6140 (1982).

Zou, Y. Inst. Phys. Conf. Ser. 63, 591 (1982). 


\section{Appendix B: Solution of diffusion to a finite sphere}

Before embarking on the derivation of the diffusion model, 1 would like to refer the reader to an excellent discussion of the general class random walk problems to which diffusion belongs. That discussion is by the esteemed astrophysicist S. Chandrasekhar (1943).

In this appendix the mathematical solution to the problem of the diffusion of $A S G_{a}$ to a precipitate is worked out in detail. To simplify the problem we will consider the diffusion to an isolated sink of radius $r_{0}$, the radius of the precipitate. The problem is spherically symmetric so that the diffusion equation describing the concentration of $\mathrm{AsGa}_{\mathrm{G}}$ can be expressed as

$$
\frac{\partial C}{\partial t}=D\left(\frac{2 \partial C}{r \partial r}+\frac{\partial^{2} C}{\partial r^{2}}\right)
$$

where $D$ is the spatially uniform diffusivity. The diffusion volume around a particular precipitate is a finite sphere of radius $R . R$ is defined so that the entire volume of the crystal is accounted for by all the precipitates, i.e.,

\footnotetext{
- Note, in order to bring $D$ out of the gradient operator it must not depend on $r$ or any other spatial variable, but this does not mean it has to be constant. D can be a function of time. This will become important later in the derivation.
} 


$$
\frac{4}{3} \pi R^{3} C_{p}=1
$$

The boundary conditions for all times, $t$, are: 1.) the concentration of AsGa at the precipitate boundary equals a constant, which usually would be the concentration in equilibrium with the precipitate phase but in our case we shall assume it to be zero, $C\left(r_{0}, t\right)=0$ (ie., an infinite sink), and 2.) the first derivative of the AsGa concentration half way between precipitates also equals zero, $\partial C(R, t) / \partial r=0$. The homogeneous boundary conditions ensure that the solutions are separable and of the form

$$
C(r, t)=\Psi(r) T(t) .
$$

Substituting this solution into the diffusion equation, and using the short hand notation that $T^{\prime}$ or $\Psi^{\prime \prime}$ is the first or second derivative of the function with respect to the appropriate variable, $t$ or $r$ respectively, Eq. B. 1 becomes

$$
\Psi T^{\prime}=D\left(\Psi^{\prime \prime}+\frac{2}{r} \Psi^{\prime}\right) T .
$$

Eq. B.4 had solutions if the time and space varying parts are equal to a constant, i.e., $\lambda^{2}$, such that

$$
\frac{T^{\prime}}{\mathrm{DT}}=\frac{\Psi}{\left(\Psi^{\prime \prime}+\frac{2}{r} \Psi^{\prime}\right)}=\lambda^{2} .
$$

The time dependent solution, assuming that it does not blow up at $t=\infty$ is then 


$$
T(t)=T_{0} \exp \left(-D \lambda^{2} t\right)
$$

The spatially dependent solution including the inner boundary condition is then

$$
\Psi(r)=\frac{1}{r} \sum_{n=1}^{\infty} A_{n} \sin \left[\lambda_{n}(r-r d]\right.
$$

so that the combined solution is

$$
C(r, t)=\frac{1}{r} \sum_{n=1}^{\infty} A_{n} \exp \left(-D \lambda_{n}^{2} t\right) \sin \left[\lambda_{n}(r-r d)\right]
$$

The constants $A_{n}$ and $\lambda_{n}$ must be determined from the initial condition and the outer boundary condition. A uniform initial concentration of $A s_{G a}, C(r, t=0)=C_{0}$, results in

$$
A_{n}=2 C_{0} \frac{\left[r_{0}+\frac{1}{\lambda_{n}} \sin \left(\lambda_{n} \Delta\right)-R \cos \left(\lambda_{n} \Delta\right)\right]}{\left[\lambda_{n} \Delta-\sin \left(\lambda_{n} \Delta\right) \cos \left(\lambda_{n} \Delta\right)\right]}
$$

where $\Delta=\left(R-r_{0}\right)$. Solving the boundary conditions at $r=R$ yields the transcendental equation for $\lambda_{n}$,

$$
\tan \left(\lambda_{n} \Delta\right)=\lambda_{n} R
$$

Experimentally, it is the total defect concentration in the layer which is measured by IR absorption, so the solution, Eq. B.8, must be 
weighted by the volume element and integrated from $r_{0}$ to $R . \bar{C}(t)$, the average concentration of $A s_{G a}$ as a function of time is

$$
\bar{C}(t)=\sum_{n=1}^{\infty} C_{n} \exp \left(-D \lambda_{n}^{2} t\right)
$$

where,

$$
C_{n}=6 C_{0} \frac{\left[\lambda_{n} r_{0}-\lambda_{n} R \cos \left(\lambda_{n} \Delta\right)+\sin \left(\lambda_{n} \Delta\right)\right]^{2}}{\lambda_{n}^{3} \Delta^{3}\left[\lambda_{n} \Delta-\sin \left(\lambda_{n} \Delta\right) \cos \left(\lambda_{n} \Delta\right)\right]^{2}} .
$$

After working out this model on my own, I discovered that a very similar but more extensive treatment of diffusion limited precipitation had been published by Ham (1958). Instead of averaging the concentration profile, he determined the change in average concentration from the atom flux at the precipitate boundary, i.e.,

$$
j(r, t)=-D \nabla[C(r, t)] .
$$

Although this is a bit more elegant the result is the same.

Typical values of $r_{0}$ and $R$ determined from electron microscopy studies of precipitates in LTMBE layers are approximately $20 \AA$ and $100 \AA$ respectively. Solving the boundary conditions with these values gives $\lambda_{1}=6.0 \times 10^{5} \mathrm{~cm}^{-1}$ and $C_{1}=$ 0.98 . Since $C_{1} \cong 1$, only the first Fourier component of the spatial solution is significant. Therefore, the time dependence diffusion process and can be described by a single exponential, 


$$
\bar{C}(t)=C_{0} \exp \left(\frac{t}{\tau}\right)
$$

where $\tau_{A s^{-1}}=\lambda^{2} \mathrm{D}$ is the time constant for annealing of the AsGa.

These solutions assume that $D$ is constant during annealing. In the case of $V_{G a}$ enhanced diffusion of $A s G_{a}$, the concentration of $V_{G a}$ is not constant hence $D$ is not constant. Like the AsGa concentration the concentration of $V_{G a}$ is also supersaturated and will decrease towards equilibrium values as the annealing progresses. Thus, the annealing process will slow down with time. This can be accounted for by introducing a time dependent diffusivity into the diffusion equation, Eq. B.1. Assuming and exponential dependence of the $\mathrm{V}_{\mathrm{Ga}}$ concentration, the diffusivity becomes,

$$
D=D_{v}^{0} \exp \left[\frac{-t}{\tau_{v}}\right]
$$

where $\tau_{v}$ is the time constant for the loss of $V_{G a}$. The spatial aspect of the diffusion problem remains the same as before but now the time dependent solutions must be modified such that

$$
\frac{\partial \bar{C}(t)}{\partial t}=\lambda^{2} D_{v}^{0} \exp \left(\frac{-t}{\tau_{v}}\right) \bar{C}(t) .
$$

Recognizing that $\lambda^{2} D_{v}{ }^{0}=\tau_{A s}{ }^{-1}$, eq. B.16 has solutions of the form,

$$
\bar{C}(t)=C_{o} \exp \left(\frac{-\tau_{v}}{\tau_{A S}}\right) \exp \left[\frac{\tau_{v}}{\tau_{A S}} \exp \left(\frac{-t}{\tau_{v}}\right)\right] .
$$




\section{References}

Chandrasekhar, S. "Stochastic problems in physics and Astronomy." Rev. Modern Phys. 15, 1 (1943).

Ham, F.S. "Theory of diffusion-limited precipitation." J. Phys. Chem. Solids 6, 335 (1958). 

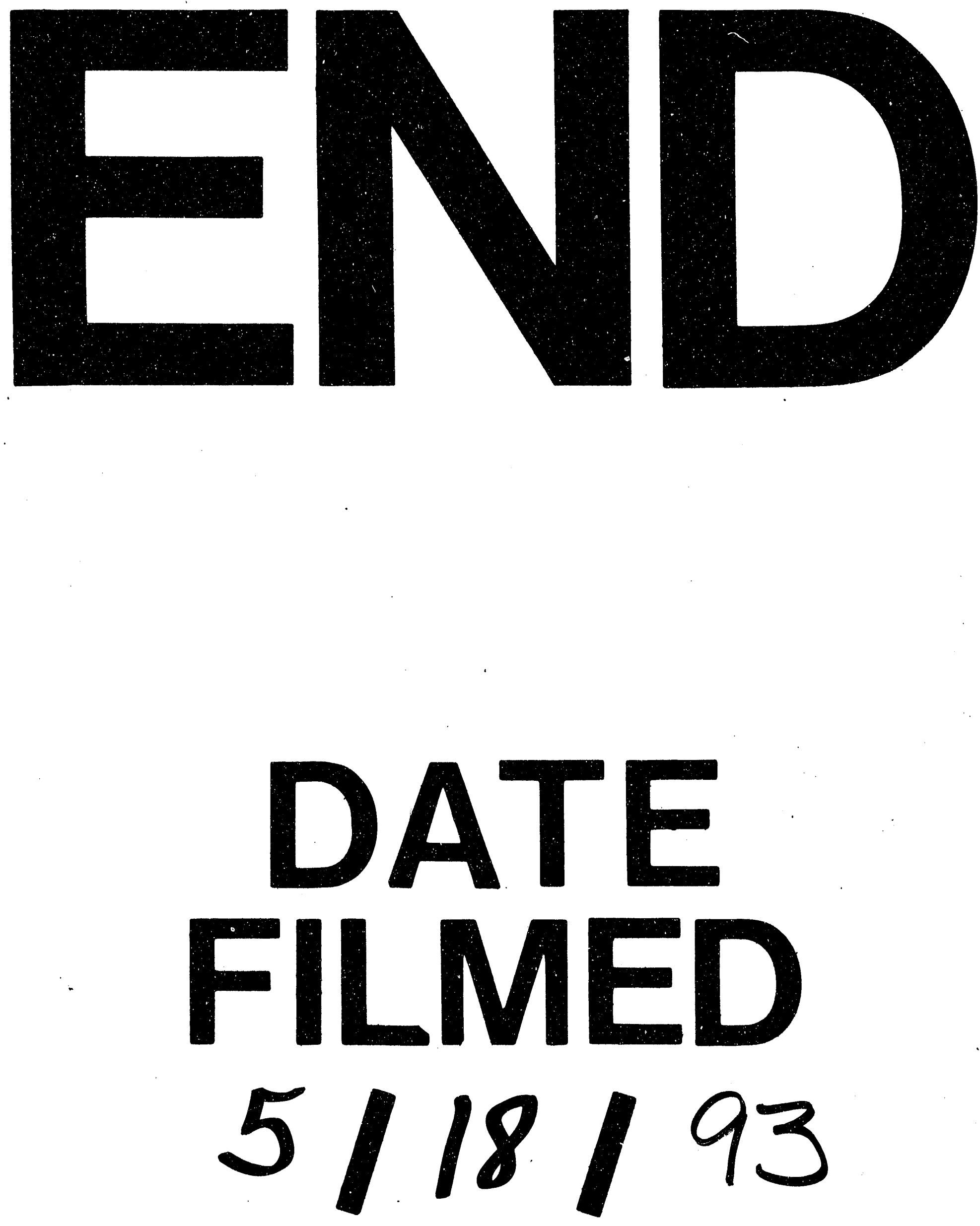
